University of Louisville

ThinkIR: The University of Louisville's Institutional Repository

Electronic Theses and Dissertations

8-2018

\title{
Binder jetting additive manufacturing process fundamentals and the resultant influences on part quality.
}

Hadi Miyanaji

University of Louisville

Follow this and additional works at: https://ir.library.louisville.edu/etd

Part of the Industrial Engineering Commons

\section{Recommended Citation}

Miyanaji, Hadi, "Binder jetting additive manufacturing process fundamentals and the resultant influences on part quality." (2018). Electronic Theses and Dissertations. Paper 3058.

https://doi.org/10.18297/etd/3058

This Doctoral Dissertation is brought to you for free and open access by ThinkIR: The University of Louisville's Institutional Repository. It has been accepted for inclusion in Electronic Theses and Dissertations by an authorized administrator of ThinkIR: The University of Louisville's Institutional Repository. This title appears here courtesy of the author, who has retained all other copyrights. For more information, please contact thinkir@louisville.edu. 
BINDER JETTING ADDITIVE MANUFACTURING PROCESS FUNDAMENTALS AND THE RESULTANT INFLUENCES ON PART QUALITY

\author{
By \\ Hadi Miyanaji \\ B.S., University of Tabriz, Iran, 2008 \\ M.S., Iran University of science \& Technology, Iran, 2011
}

\begin{abstract}
A Dissertation
Submitted to the faculty of the

J. B. Speed School of Engineering of the University of Louisville In Partial Fulfillment of the Requirements for the Degree of
\end{abstract}

\author{
Doctor of Philosophy \\ In Industrial Engineering \\ Department of Industrial Engineering \\ University of Louisville \\ Louisville, Kentucky
}

August 2018 
Copyright 2018 by Hadi Miyanaji

All rights reserved 

BINDER JETTING ADDITIVE MANUFACTURING PROCESS FUNDAMENTALS AND THE RESULTANT INFLUENCES ON PART QUALITY

By

Hadi Miyanaji

A Dissertation Approved on

Jun 8, 2018

by the following Dissertation Committee:

Dissertation Director: Dr. Li Yang

Dr. Kevin Chou

Dr. Gail W. DePuy

Dr. Delaina A. Amos 


\section{ACKNOWLEDGMENTS}

I would like to express my sincere gratitude to my advisor, Dr. Li Yang, for giving me an opportunity to conduct my research under his guidance. It has been great honor to have known him and work for him. Dr. Yang has always been the source of creative ideas and insightful advice without imposing his opinions which provided me a wonderful atmosphere to enjoy the freedom in performing my dissertation.

I also would like to extend my appreciation to Dr. Delaina A. Amos, Dr. Gail W. DePuy, Dr. Qian Zhao, and Dr. Thad Druffel for the scientific conversations and insightful suggestions about my research as well as the tremendous help for conducting different experimentations.

I am truly thankful to all my friends and colleagues for their support and friendship. I also acknowledge the kind assistance and contributions of the staff of the Rapid Prototyping Center (RPC) at University of Louisville.

Last but not least, I would like to thank my parents whose love and guidance are with me

in whatever I pursue; and my best-friend loving wife, Fereshteh, who provided me unending support and inspiration throughout these years. 


\section{ABSTRACT \\ BINDER JETTING ADDITIVE MANUFACTURING PROCESS FUNDAMENTALS AND THE RESULTANT INFLUENCES ON PART QUALITY \\ Hadi Miyanaji}

June 20, 2018

Binder jetting additive manufacturing (BJ-AM) technology is a process by which a part is fabricated layer by layer through depositing liquid binder in the designated regions of each layer until the feature is complete. Due to the use of binder in geometry generation, this process possesses various advantages over the other additive manufacturing technologies including the ability to create colored objects via colored binders, lower cost, lack of heat-induced defects, and its potential capability to print a variety of materials such as metals, sands and ceramics. Although the application of the binder jetting process has been recently reported by different researchers in areas such as dentistry, biomedicine, aerospace, foundry, and automobile, the adoption of this technology in the manufacturing industry has been slower compared to other AM processes. This could be largely attributed to the uncertainties involved in the feature fabrication process (e.g. lack of qualitative/quantitative relationships between process set up characteristics and the quality of printed features) which mainly arises from the lack of adequate insight into the fundamentals of this technology. As a consequence, the fabrication of components, particularly if printing of new materials is intended, and development of binder jetting is 
currently based upon trial and error approach. Therefore, this research project aims to take a closer look at this technology to establish comprehensive understanding of the process characteristics by thoroughly investigating the feature fabrication in Binder Jetting process. For this purpose, the feature fabrication process in BJ-AM technology is closely monitored, and the effect of different parameters on part quality are investigated. New physics-based models are developed for describing the process characteristics at different stages. In order to predict the optimal binder saturation level, a new model is developed to describe the binder-powder bed interaction in the equilibrium state. For modeling the binder-powder bed interaction in the dynamic state, the effect of the different parameters (e.g. droplet impact velocity, the mean particle size and particle size distribution) on the feature formation process are experimentally explored. Finally, a model describing the binderpowder bed interaction in the dynamic state is developed to predict the profile of the powder saturated area by a single binder droplet. 


\section{TABLE OF CONTENTS}

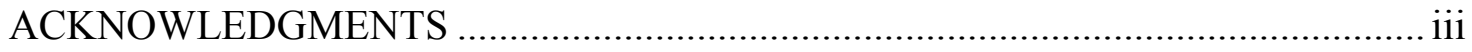

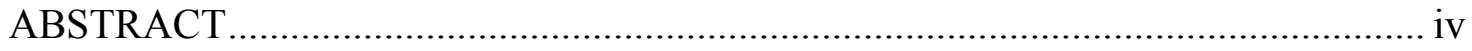

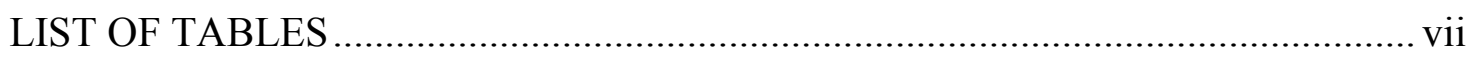

LIST OF FIGURES …................................................................................. viii

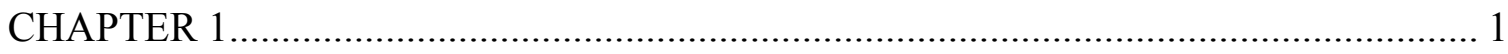

RESEARCH MOTIVATION AND PROBLEM STATEMENT ............................... 1

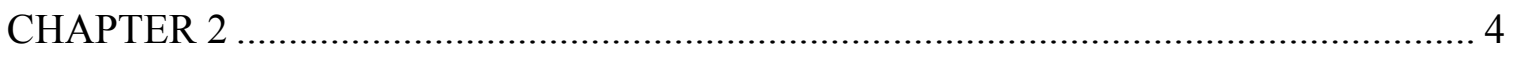

LITERATURE REVIEW ............................................................................... 4

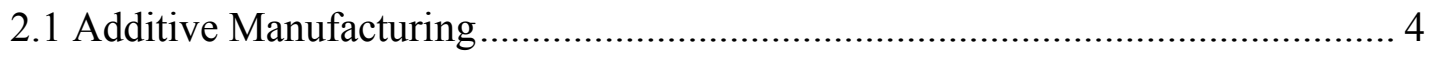

2.2 Binder Jetting additive manufacturing ..................................................... 5

2.3 Determining parameters of 3D-printed part quality ...................................... 7

2.3.1 Process related parameters .................................................................. 7

2.3.2 material related parameters .............................................................. 18

2. 4 Feature Formation in Binder Jetting Process ........................................... 22

2.4.1 Modeling of droplet-porous meduim interaction................................. 23 
CHAPTER 3

3. 1 Introduction 54

3. 2 Feature formation in Binder Jetting Process in the most fundamental level ..... 54

3. 3 Developing a physics-based model for the binder/powder bed interaction in the equilibrium phase 57

3. 4 Investigating the effect of different parameters on binder-powder bed interaction in the dynamic phase. 60

3.5 Developing a theoretical model to describe the droplet-powder bed interaction in the dynamic phase 60

CHAPTER 4 63

A NEW PHYSICS-BASED MODEL FOR EQUILIBRIUM SATURATION
DETERMINATION IN BINDER JETTING ADDITIVE MANUFACTURING

PROCESS 63

4.1 Introduction 63

4.2 Modeling of binder saturation 67

4.3 Experimental Procedure. 76

4.4 Results and discussion 80

4.5 Conclusions 88

CHAPTER 5 90 


\section{EFFECT OF PRINTING SPEED ON QUALITY OF PRINTED PARTS IN BINDER}

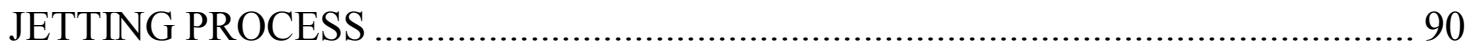

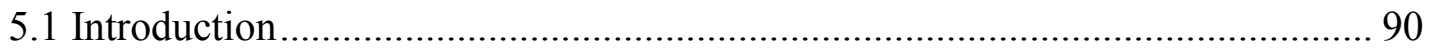

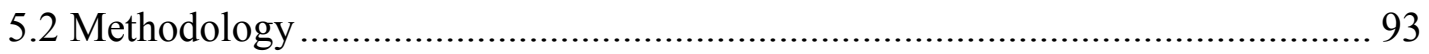

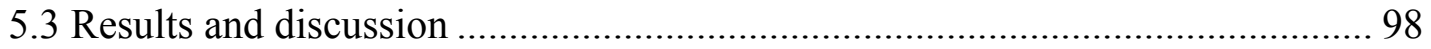

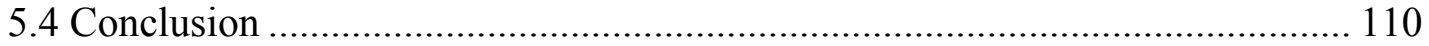

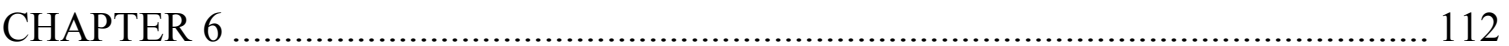

EFFECT OF POWDER MATERIAL CHARACTERISTICS ON QUALITY OF

PARTS FABRICATED VIA BINDER JETTING PROCESS................................. 112

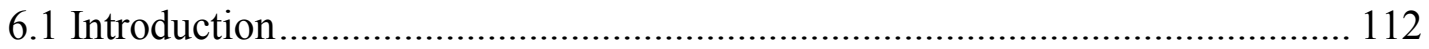

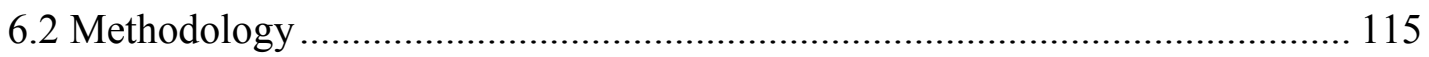

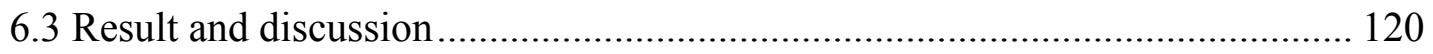

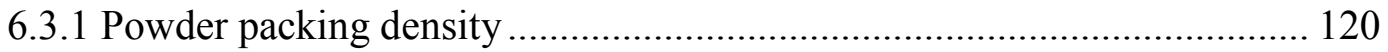

6.3.2 Powder/binder interaction and accuracy .......................................... 121

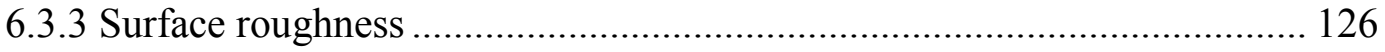

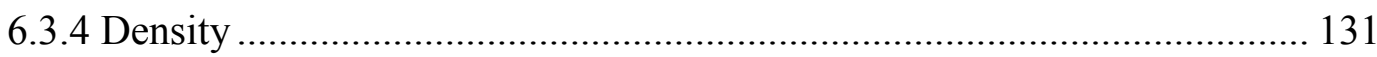

6.3.5 Microhardness of the sintered parts ..................................................... 134

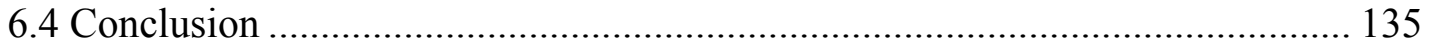

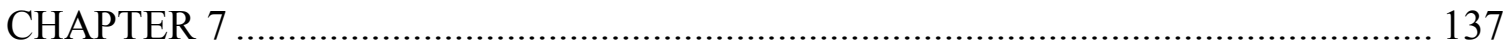




\section{BINDER DROPLET PROFILE PREDICTION AFTER COMPLETE}

PENETRATION INTO A POWDER BED............................................................ 137

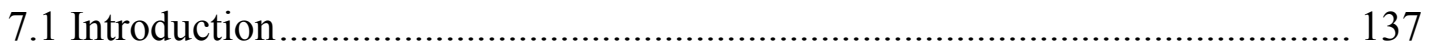

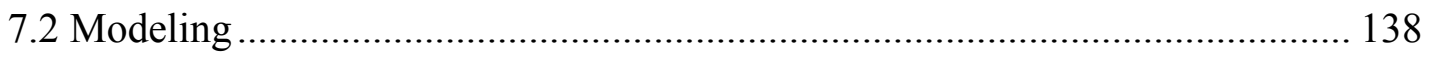

7.3 Methodology for the model validation ...................................................... 147

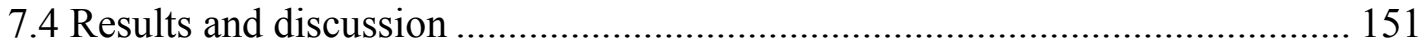

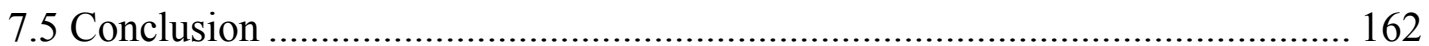

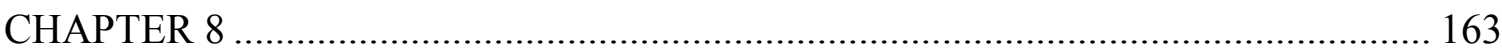

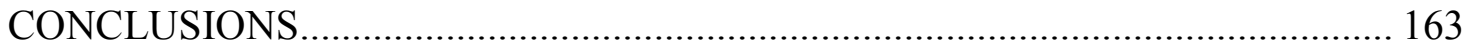

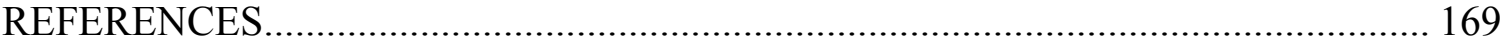

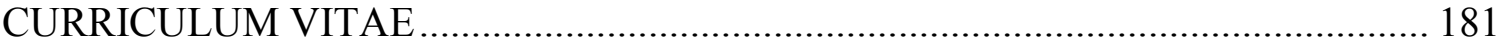




\section{LIST OF TABLES}

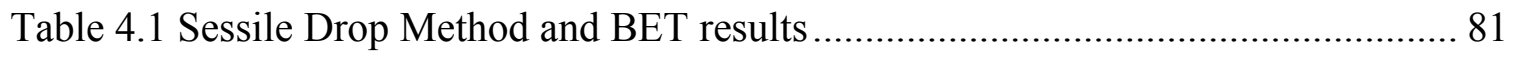

Table 4.2 Effect of optimal saturation level on the dimensional accuracy of Ti-6Al-4V

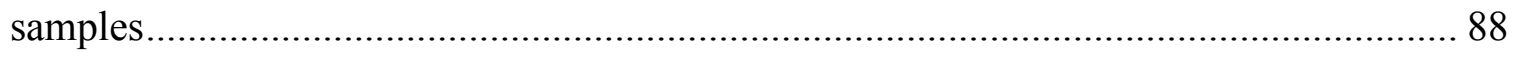

Table 6.1 316L stainless steel powders used for the experiments .............................. 115

Table 6.2 Printing parameters used for the M-Lab BJ-AM system ............................. 119

Table 7.1 Experimental parameters needed for impact driven maximum spreading ..... 151

Table 7.2 Experimentally measured contact angles.............................................. 151

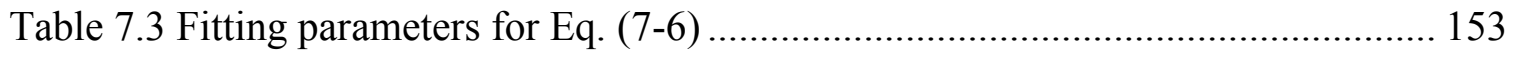

Table 7.4 Mean pore radius and pore density for different powder beds ..................... 154

Table 7.5 The measured permeabilities for different powder materials ....................... 157

Table 7.6 Predicted values for maximum spreading and penetration depth ................. 158 


\section{LIST OF FIGURES}

Fig. 2.1 Binder Jetting geometry creation process .................................................. 6

Fig. 2.2 Schematic of binder jetting process for the ExOne M-Lab machine [14] .......... 8

Fig. 2.3 3D printed TiNiHf mesh structure with $50 \mu \mathrm{m}$ printing layer thickness and 55\%

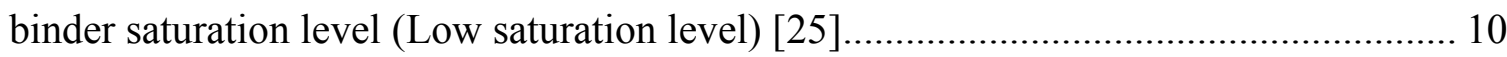

Fig. 2.4 3D printed dental porcelain samples using different saturation levels, (a) 45\%;

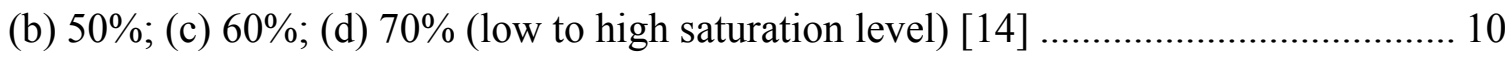

Fig. 2.5 Printed parts after curing [13] ............................................................... 11

Fig. 2.6 Effect of different factors on part accuracy in z direction and mechanical

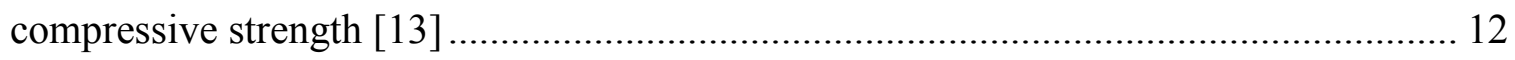

Fig. 2.7 Effect of power level on the compressive strength of the green parts [14]........ 13

Fig. 2.8 Surface plot of green part strength vs drying time and power level................... 14

Fig. 2.9 Printed wire width measured by optical microscope [25] .............................. 16

Fig. 2.10 Different printing layer thickness and binder spreading cases [25] ................ 16

Fig. 2.11 Comparison of compressive strength in samples with different layer thickness in

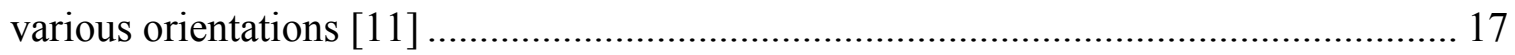

Fig. 2.12 Different printing orientation of samples [11] ........................................ 17

Fig. 2.13 Dependency of flowability $\left(\mathrm{ff}_{\mathrm{c}}\right)$ on median particle size [32] ........................ 19

Fig. 2.14 The effect of particle (grain) size on packing density [47] ............................ 20 
Fig. 2.15 The effect of particle shape on shrinkage of stainless steel during conventional

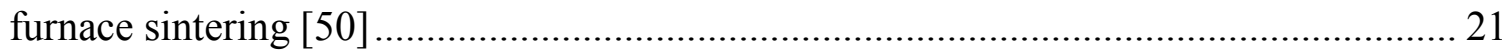

Fig. 2.16 Schematic diagram of binder migration in the powder bed........................... 24

Fig. 2.17 Local contact angle between liquid binder and a particle in the powder bed.... 26

Fig. 2.18 Typical capillary pressure-wetting fluid saturation curves [24] ...................... 27

Fig. 2.19 The model for equilibrium of a liquid binder in a hydraulic feature [24] ........ 29

Fig. 2.20 Two examples of printed features for empirical equilibrium saturation

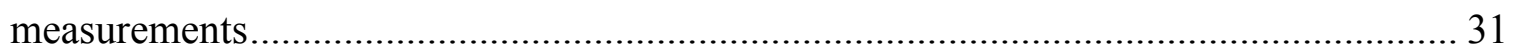

Fig. 2.21 Equilibrium state of two partially saturated pores ...................................... 32

Fig. 2.22 The definition of the system. (b) The general equilibrium situation. (c) Final equilibrium situation in the absence of gravity, for $\Theta<90^{\circ}$ and for $\theta>90^{\circ}$ [57] f........ 34

Fig. 2.23 The radial capillary. (b) Penetration without hysteresis. (c) Penetration with a

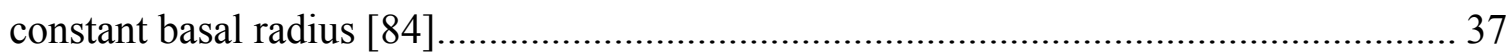

Fig. 2.24 Cross section (upper) and top view (lower) of the geometry for the model of the

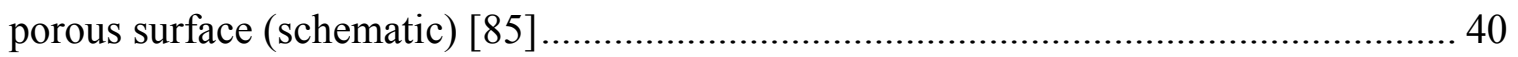
Fig. 2.25 (a) Schematic representation of the decreasing drawing area (b) the constant drawing area (the thickness of the remnant film in case b (2) is exaggerated for illustrative purpose). In (a), rsc $=r d$, both decrease with time; in $b(1), r s c=r d=$ constant; and in b (2) rsc decreases with time and $\mathrm{rd}=$ constant [85] 41

Fig. 2.26 Characteristic prediction for constant and decreasing contact area limiting cases [53]. 43 
Fig. 2.27 (a) The drop and the thin porous medium prior to contact (b) initial spreading over the surface of the porous medium, which may coincide with (c) initial penetration into the porous medium (d) basal penetration (e) a "halo" (f) complete penetration [56] 46 Fig. 2.28 Schematic representative element of an interconnected pore geometry [53] ... 48 Fig. 2.29 Axes definition for the spread and absorption of a sessile droplet at time $\mathrm{t}$ [86]

Fig. 2.30 Spreading and penetration behavior studied by Starov et al $[89,90,93] \ldots \ldots \ldots . .50$

Fig. 2.31 Geometry of droplet penetration by a porous medium [94] .......................... 51

Fig. 2.32 Illustrating A12O3 particles entering a water droplet ................................. 53

Fig. 3.1 Droplet deposition pattern in Binder Jetting process.................................... 55

Fig. 3.2 Equilibrium condition between binder and powder bed................................ 56

Fig. 3.3 Binder droplet permeation in powder bed .................................................. 57

Fig. 3.4 Schematic of single track and multiple tracks experiments ............................ 59

Fig. 3.5 Schematic of feature formation in Binder Jetting Process .............................. 59

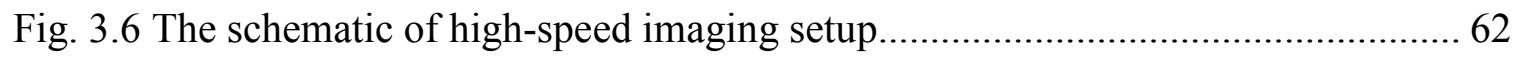

Fig. 4.1 Schematic presentation of a binder droplet on the powder surface .................... 64

Fig. 4.2 Equilibrium state in the pore scale (Pc: capillary pressure) …....................... 65

Fig. 4.3 Phase Diagrams for imbibition: a) large aspect ratio; b) small aspect ratio [114]

Fig. 4.4 Schematic presentation of a binder droplet pattern inside powder bed (binder droplet after reaching equilibrium state); a) assuming small pore size, b) assuming large pore size 
Fig. 4.5 SEM image of binder (deposited by a micropipette) and 420SS interaction in the

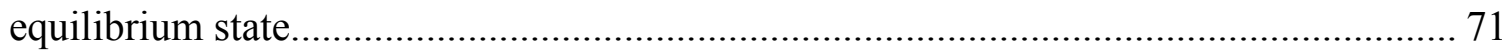

Fig. 4.6 Schematic representation of a droplet penetrating a uniform porous medium.... 73 Fig. 4.7 The morphology of a) 420 Stainless steel b) Ti-6Al-4V (grade 23) ................. 77

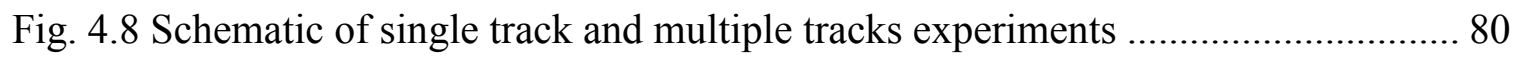



Fig. 4.10 SEM micrograph of powder materials used, a) Ti-6Al-4V b) 420 SS ........... 82

Fig. 4.11 Equilibrium saturation. The error bars indicate the standard deviation for each



Fig. 4.12 schematic of parts printed with different in-process binder curing parameters a) insufficient drying parameters b) appropriate drying parameters (the dashed arrows indicate the potential binder migration paths inside the powder bed) .......................... 86 Fig. 5.1 The designed geometry for printing samples and part configuration in the powder

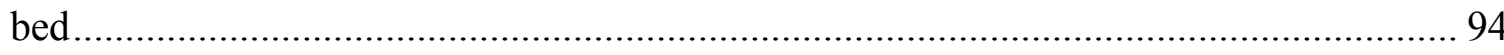

Fig. 5.2 Schematic of single track and multiple tracks experiments ............................ 96

Fig. 5.3 The morphology of 420 Stainless Steel powder material ................................ 97

Fig. 5.4 Printed features with different printing speeds (a) $20 \mathrm{~mm} / \mathrm{sec}$ (b) $100 \mathrm{~mm} / \mathrm{sec}$

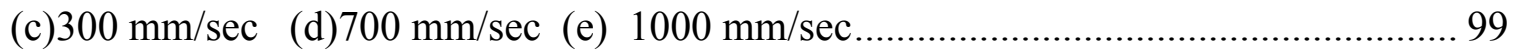

Fig. 5.5 Variation of the slot accuracy with printing speed and part orientation........... 100

Fig. 5.6 Schematic graph of a droplet release from the printhead .............................. 102

Fig. 5.7 Sequence of droplet impact phenomenon on the powder bed surface ............. 103

Fig. 5.8 Schematic representation of a sample printed with two different printing speeds 
Fig. 5.9 Effect of printing speed on dimensional acuuracy of single-track samples ..... 105 Fig. 5.10 Dimensional accuracy of multiple-track samples fabricated at various printing speeds 105

Fig. 5.11 Printing speed effect on accuracy of cubic parts ..................................... 106

Fig. 5.12 Schematic of feature formation inside a layer ........................................ 108

Fig. 5.13 Saturation variation with printing speed for cubic samples ........................ 110

Fig. 6.1 Schematics of BJ-AM process steps.................................................... 113

Fig. 6.2 SEM images of 316L SS powder with different size distributions a) powder type I with 5-25 size distribution b) powder type II with 15-45 size distribution c) powder

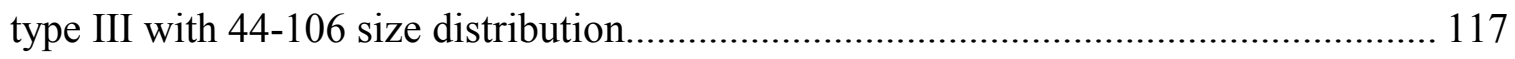

Fig. 6.3 The schematic of high-speed imaging setup............................................ 117

Fig. 6.4 Packing density of the different powder types with the polydispersity index ... 121 Fig. 6.5 The formed features resulted from the interaction of a single binder droplet with different powder types (a) powder type I $(14 \mu \mathrm{m})$ (b) powder type II $(31 \mu \mathrm{m})$ c) powder type III $(78 \mu \mathrm{m})$ 122

Fig. 6.6 Depth and diameter of single drop features vs mean particle size of powdered materials 123 Fig. 6.7 Interaction of a binder droplet with different powder types at the end of impact-

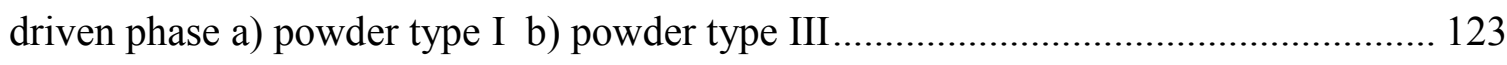
Fig. 6.8 The effect of mean particle size on dimensional accuracy of printed parts ..... 126 Fig. 6.9 Schematic representation of fabrication platform and defined directions ........ 127 Fig. 6.10 Surface roughness of fabricated green specimens in X and Y directions ....... 127 Fig. 6.11 Schematic of 2D feature formation within a layer ................................ 128 
Fig. 6.12 The effect of the raster scanning strategy for binder delivery on the surface roughness of the green parts a) sample fabricated with the powder type I b) sample made

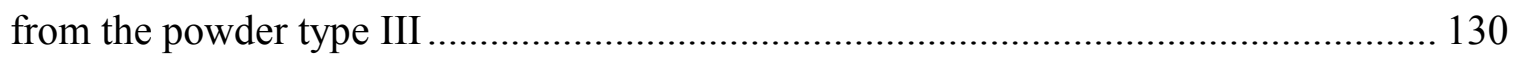
Fig. 6.13 Surface roughness of specimens after sintering in $\mathrm{X}$ and $\mathrm{Y}$ directions ........... 131 Fig. 6.14 Effect of particle size on green part density .............................................. 132 Fig. 6.15 Effect of particle size distribution on the density of the sintered parts .......... 133 Fig. 6.16 SEM micrographs of sintered specimens a) type I b) type II c) type III ....... 134 Fig. 6.17 Effect of the mean particle size on hardness of the sintered samples............. 135 Fig. 7.1 Schematic representation of droplet penetration into the powder bed a) Vertical penetration rate $(\mathrm{VP})>>$ radial spreading rate over the surface $(\mathrm{VS})$ b) VP $\sim$ VS....... 142 Fig. 7.2 Schematic illustration of the sequence of events that occurs after a droplet impacts on a porous substrate a) Vertical penetration rate (VP) $>>$ radial spreading rate

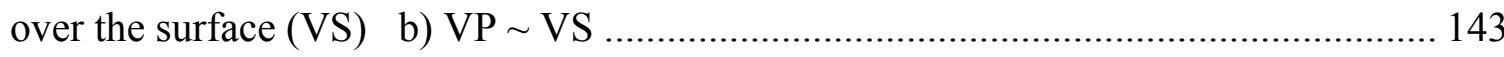

Fig. 7.3 The schematic of high-speed imaging setup.................................................... 148

Fig. 7.4 Volumetric pipet used for measuring the surface tension of the binder ............ 148 Fig. 7.5 Schematic diagram of the set up for permeability measurement...................... 149 Fig. 7.6 Diameter of a droplet spreading on the surface of different powder beds versus time 152 Fig. 7.7 Real time images of characteristics of the droplet interacting with powder material type I from impact (image 1) to complete penetration (image 8) 153 Fig. 7.8 Instantaneous spreading droplet radius (red color) and the equilibrium droplet radius (blue color) for powder beds a) type I b) type II c) type III 156 
Fig. 7.9 schematic of a formed droplet profile inside the powder after complete penetration...

Fig. 7.10 Maximum diameter and depth of droplet profile inside the powder bed from the developed model and the experiments. 160

Fig. 7.11 Instantaneous spreading droplet radius and the equilibrium droplet radius for powder beds type I, II, and III 161 


\section{CHAPTER I}

\section{RESEARCH MOTIVATION AND PROBLEM STATEMENT}

Binder Jetting additive manufacturing process possesses unique advantages compared to other additive manufacturing processes as it uses a liquid binder for geometry creation that does not involve intensive heat during the fabrication process. Some of such advantages include the ability to create colored objects through colorful liquid binders, high speed, reasonably low cost, the capability to print variety of materials (e.g. metals, polymers, and ceramics), lack of heat-related defects in the printed parts, scalability as well as straightforward work principles. Furthermore, in this technology there is no need to design support structures for fabricating overhanging features. In comparison, many other AM processes require additional structures for part anchoring or heat dissipation purposes. Such advantage of binder jetting effectively eliminates the effort of printing and removing support structures, which sometimes could be a difficult, time-consuming and expensive task to perform. Due to these favorable characteristics, binder jetting process has received a lot of attention from various industries such as dentistry, biomedical, automobile, foundry, and aerospace in the past few years and several research works have been reported on applying this method to fabricate parts for different applications. 
Despite its great potential, the overall rate of industrial adoption of binder jetting additive manufacturing process has been considerably slower compared to many other additive manufacturing processes. This is largely due to the lack of adequate understanding into the fundamentals of this technology. The process development and the quality of parts of the binder jetting technology is significantly limited by various factors including insufficient understanding of binder/powder bed interaction, the lack of predictive models for the binder/powder bed interaction and part property prediction, the lack of systematic guidelines for choosing proper process parameters for each set of new materials, and inadequate knowledge about the effect of different process parameters and material (powder and binder) characteristics on part quality.

Therefore, in order to overcome these issues and accelerate the development and adoption of the binder jetting technology, clear understanding about the fundamentals of this technology is needed, which is the focus of this research. The ultimate goal of the research is to enable the fabrication of structures and parts with high integrity and quality in terms of both mechanical properties and dimensional accuracy through fundamental understanding of the process characteristics. This goal will be accomplished by breaking it down into multiple objectives:

1. Establish quantitative theories for the interaction between binder and powder bed both in the static (i.e. equilibrium) and dynamic states;

2. Evaluate the effect of process parameters on the process characteristics of binder jetting;

3. Establish a deposited droplet profile in the powder bed based on the developed models; 
4. Establish quantitative/qualitative correlations between the process designs and the mechanical and dimensional properties of the fabricated parts. 


\section{CHAPTER 2}

\section{LITERATURE REVIEW}

\subsection{Additive Manufacturing}

Additive Manufacturing (AM) technology refers to as processes in which a digital 3D model is used to generate a feature by joining the built materials layer upon layer. Based on the type of built materials, the deposition technique, and the way the materials are joined together, AM processes are classified into seven categories including Photopolymerization, Material jetting, Binder jetting additive manufacturing, Material extrusion, Powder Bed Fusion, Sheet Lamination, Direct Energy Deposition according to ASTM F2792-12a [1]. $\mathrm{AM}$ is receiving unprecedented attention from various industries. In the recent years, the additive manufacturing market, including the all additively manufactured products and corresponding services worldwide, has grown impressively [2-4]. Some of the recent improvements in these processes contributing to this rapid growth include their increasing capability to fabricate end-use products, the enhancements of the speed and performance of additive manufacturing machinery, an increasing range of built materials. These advancements in turn inspire the further implementation of AM technology in different industries around the world $[2,5,6]$. Compared to other AM processes, the overall speed of adoption for Binder Jetting additive manufacturing technology has been significantly slower largely owing to lack of clear understanding the fundamentals of the technology 
and quantitative relationship between the process setup and the achievable outcomes. Therefore, in the current research, Binder Jetting additive manufacturing process will be thoroughly investigated and analyzed in order to provide more quantitative insight of the process in the attempt to facilitate the industrial adoption of this promising technology.

\subsection{Binder Jetting additive manufacturing}

Binder Jetting additive manufacturing technology, initially developed in the early 1990s at MIT [7], is an additive manufacturing process which is based on a liquid binder deposition in the designated regions acquired from a digital 3D model. As shown in Fig. 2.1, after spreading a layer of powder material a printhead selectively deposits certain amount of binder droplets onto the surface of the powder bed. Thereafter, the powder bed surface is exposed to set amount heating via an external radiation heat source (e.g. infrared heaters) in order to partially cure the binder within the regions saturated by the binder and to establish minimum mechanical strength. The mechanical strength within each layer which results from partial evaporation of water content existed in the binder formulation is required to ensure the feature structural integrity and desired dimensional accuracy during printing. After the drying/curing process, a new layer of powder is applied, and the process repeats until the entire part (so called green part) is printed. The fabricated green parts usually have relatively low strength and therefore will be subjected to further postprocesses such as infiltration or sintering in order to achieve adequate mechanical strength.

Binder Jetting is of great potential to be adopted in different industries for diverse applications. Various research-based works have been reported on applying this 
technology in different applications such as biomedicine [8-11], aerospace [12], dentistry $[13,14]$, foundry [15] and automobile [16].

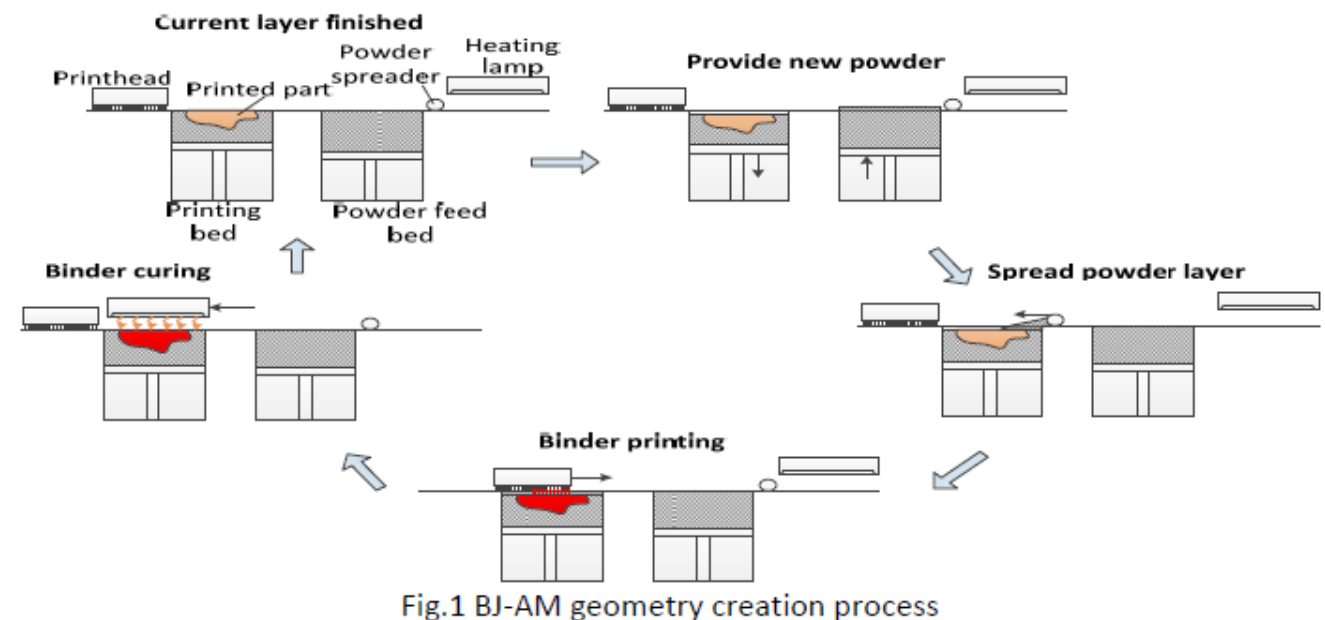

Fig. 2.1 Binder Jetting geometry creation process

Due to the use of a liquid binder for geometry generation, Binder Jetting additive manufacturing possesses various unique advantages over other AM technologies including the ability to create colored objects via colored binders, lower cost, usually higher speed, lack of heat-induced defects, and it potential capability to print a variety of materials such as metals, sands and ceramics [17]. In addition, the Binder Jetting process is regarded as the most scalable among all AM processes in that the build size can be theoretically extended as required by expanding the axial distances over which the printhead and build platform move [18].

However, the inadequate mechanical characteristics of the printed green parts usually necessitate multiple post-processing steps. The selection of the post-processing methods in Binder Jetting differs rely mostly on the printed material and application of the fabricated features. Polymer materials printed by Binder Jetting are typically infiltrated with a secondary infiltrant material to improve mechanical strength or color. Metals and 
ceramics green parts require a thermal post-processing step to densify and strengthen the printed parts via sintering $[19,20]$. Another limitation of this process is the lack of theoretical models for predicting the quality of 3D-printed part and also deep understanding of the process fundamentals which leads to considerable uncertainties regarding the accuracy and strength of fabricated parts $[20,21]$.

\subsection{Determining parameters of 3D-printed part quality}

Although Binder Jetting technology is of straightforward work principles compared to other AM processes, there exist multiple factors that might significantly contribute to the structural integrity and quality of the printed features. These parameters can be divided into two main groups, the process related parameters and the material related parameters.

\subsubsection{Process related parameters}

\subsubsection{Spreading speed}

As shown in Fig. 2.2, spreading of powder layers in Binder jetting process is accomplished via a clockwise-rotating roller which traverses back and forth over the build platform. Therefore, spreading speed is defined as the rate at which the rotating roller moves forward for spreading a layer of powder (i.e. the rate at which a layer of powder is spread).

Inappropriate spreading speed would result in uneven and non-uniform spreading with undesired large porosity in the final product which eventually leads to lower mechanical strength [13]. Lower spread speed is always desired for uniform powder spreading and as a consequence the quality of printed parts. However, it could significantly 
increase the printing time. Due to the large Van der Wall's forces, finer particles $(<5 \mathrm{um})$ do not tend to spread well and require a relatively slower spread speed, whereas relatively higher spread speed could be set for coarse particles [22].

The morphology of the particles also affects the spreading of the material. Faceted and anisotropic particles will stick to each other, creating more friction compared to spherical particles. It has been shown in $[14,23]$ how irregular particle shape and wide range of particle size distribution could greatly lower the powder flowability. The flow characteristics directly affects the density of the final part. If a powder does not spread well, it does not pack well, resulting in a lower density [7].

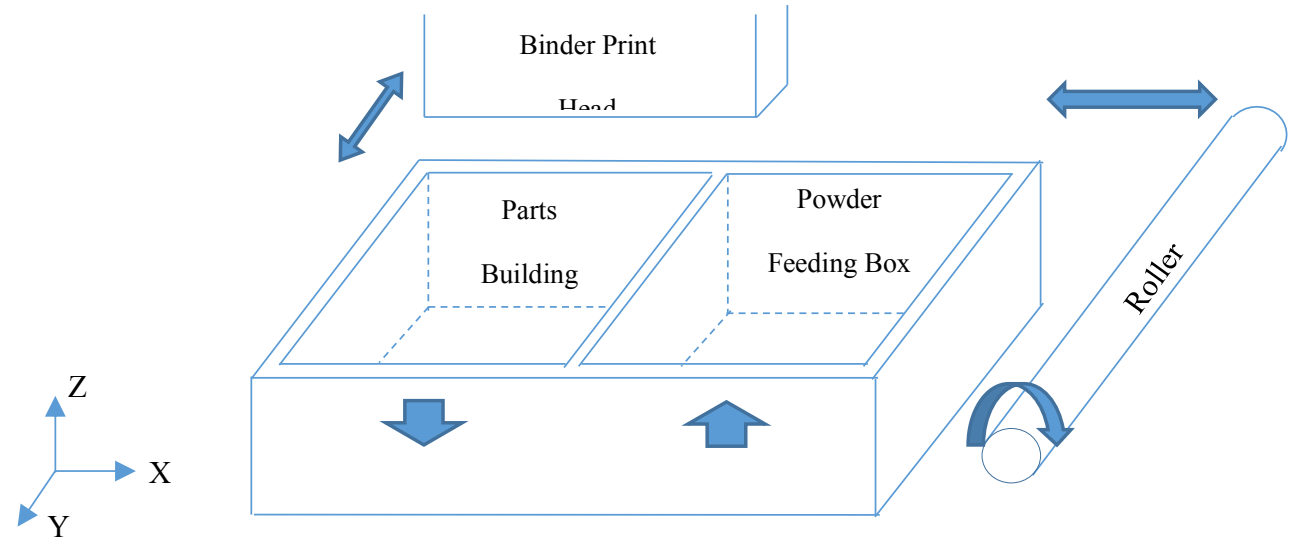

Fig. 2.2 Schematic of binder jetting process for the ExOne M-Lab machine [14]

\subsubsection{Binder saturation}

Binder saturation level is defined as the amount of the binder which is deposited through printhead. It is determined by calculating the theoretical percentage of void space $\left(\mathrm{V}_{\text {air }}\right)$ in the print material that is filled with binder ( $\left.\mathrm{V}_{\text {binder }}\right)$ following Eq.(1-1)-(1-2), in which PR stands for the packing density of the powder bed and $\mathrm{V}_{\text {envelope }}$ stands for the 
volume of a defined envelope. It is worth mentioning that packing density specifies how much void space exists in the print material after spreading.

$$
\begin{gathered}
\text { Desired saturation level }=\mathrm{V}_{\text {binder }} / \mathrm{V}_{\text {air }} \\
\mathrm{V}_{\text {air }}=(1-\mathrm{PR} 100) * \mathrm{~V}_{\text {envelope }}
\end{gathered}
$$

Optimal saturation amount needs to be used in printing process to ensure the good mechanical strength and desired dimensional accuracy and structural integrity. Both lower saturation and higher saturation levels will introduce inaccuracy and diminished mechanical properties in the printed parts. While with low saturation there won't be sufficient binder to join the powders particles together and create a strong bond between successive layers (Fig. 2.3), with higher saturation levels the excess binder will permeate out of the designated boundaries as shown in Fig. 2.4. In addition, two other phenomena that might be observed if excessive amount of binder is used for printing are related to migration of binder within the powder bed, so called bleeding and feathering in the literature $[13,24]$. Bleeding is the macroscopic flow of binder within the features printed with high saturation levels that usually hang off the bottom of a large part. In this case, printing successive layers on top of excessive under-cured binder would decrease accuracy in the bottom section of the part more than top layers (which are printed later in the process) probably due to gradually accumulated weights from the top layers [13]. Feathering is the microscopic spread of binder from the point of impact and is observed as a broadening printed geometry [24]. An example of feathering phenomenon has been shown in Fig. 2.5. 


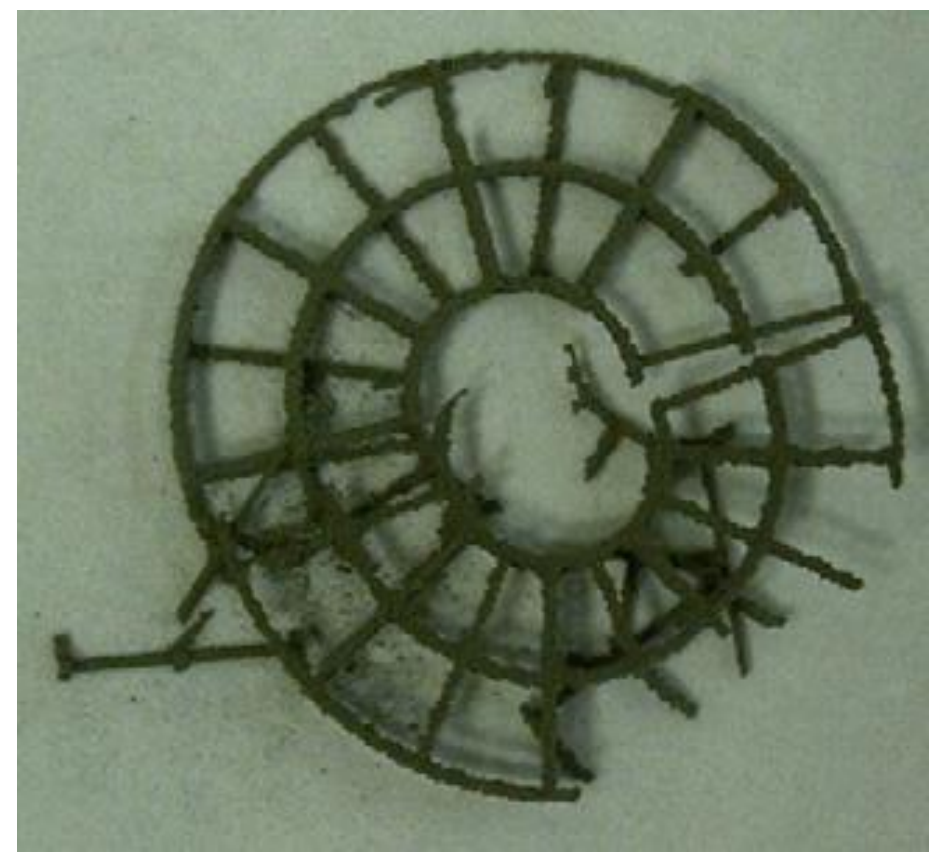

Fig. 2.3 3D printed TiNiHf mesh structure with $\mathbf{5 0} \mu \mathrm{m}$ printing layer thickness and $55 \%$ binder saturation level (Low saturation level) [25]

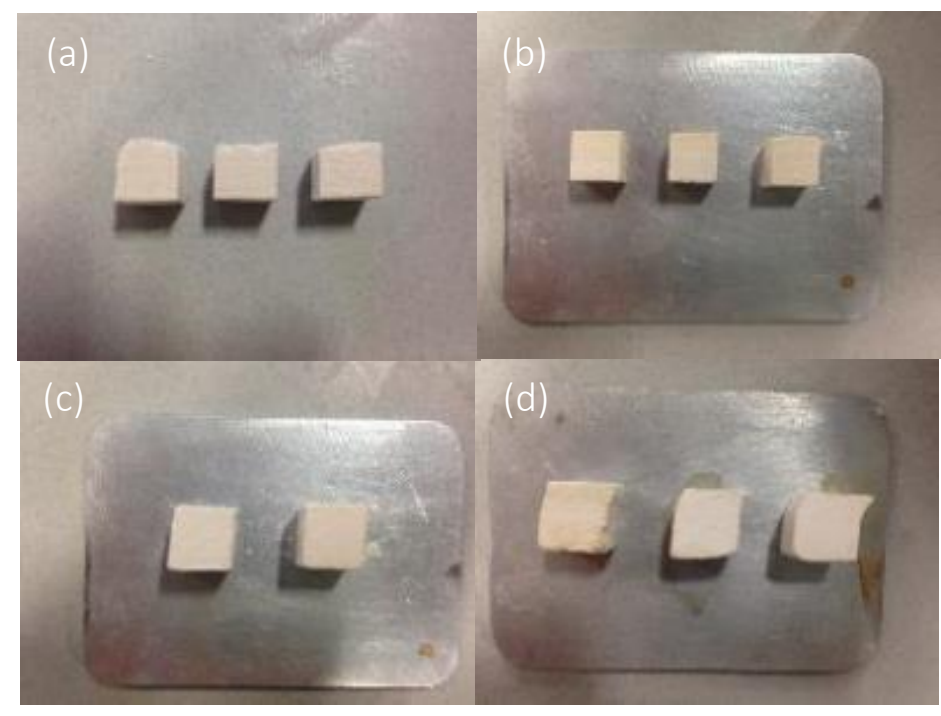

Fig. 2.4 3D printed dental porcelain samples using different saturation levels, (a) $45 \%$; (b) 50\%; (c) 60\%; (d) 70\% (low to high saturation level) [14] 


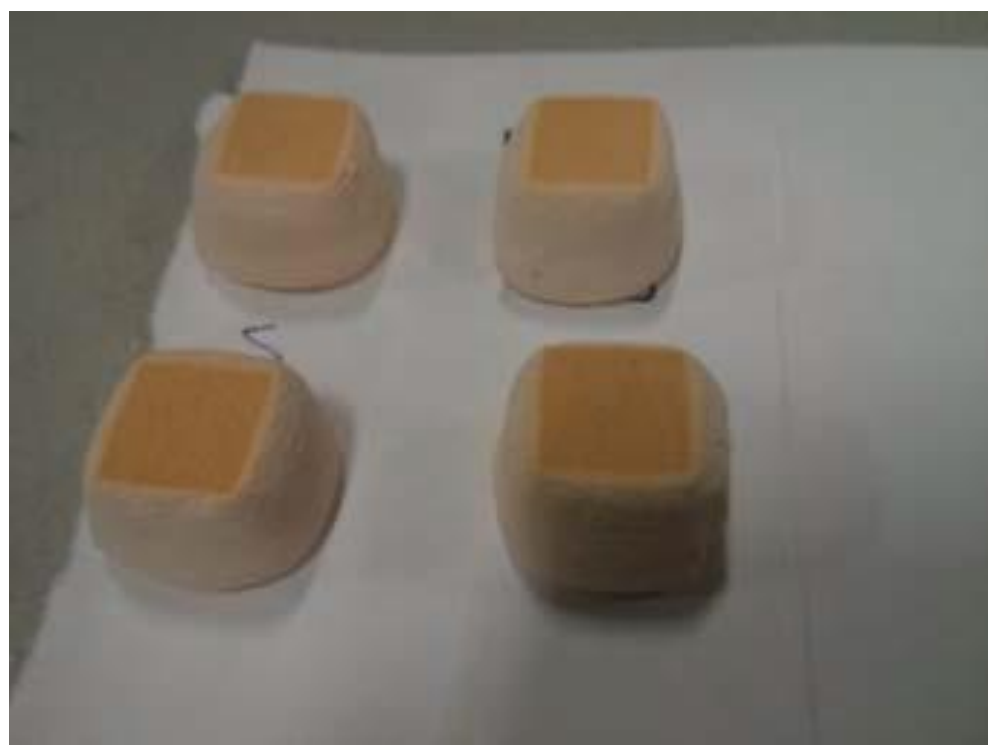

Fig. 2.5 Printed parts after curing [13]

The effect of saturation amount, along with other parameters, on part dimensional accuracy and strength has been investigated by multiple researchers $[11,13,25-30]$. In the preliminary study conducted by the author [13], a systematic experimental based study was carried out to establish the relationship between various process parameters of ExOne MLab binder jetting system (including binder amount, drying time, drying power, powder spread speed) and the qualities of the green parts and finished parts, which in turn provides insights into the fundamental principles of the process. It was demonstrated that how changing saturation level and its interaction with other parameters would affect the part structural integrity (Fig. 2.6). In this study, a set of guidelines are provided for adjusting the printing parameters for new material development and the better understanding of the binder jetting processes. 


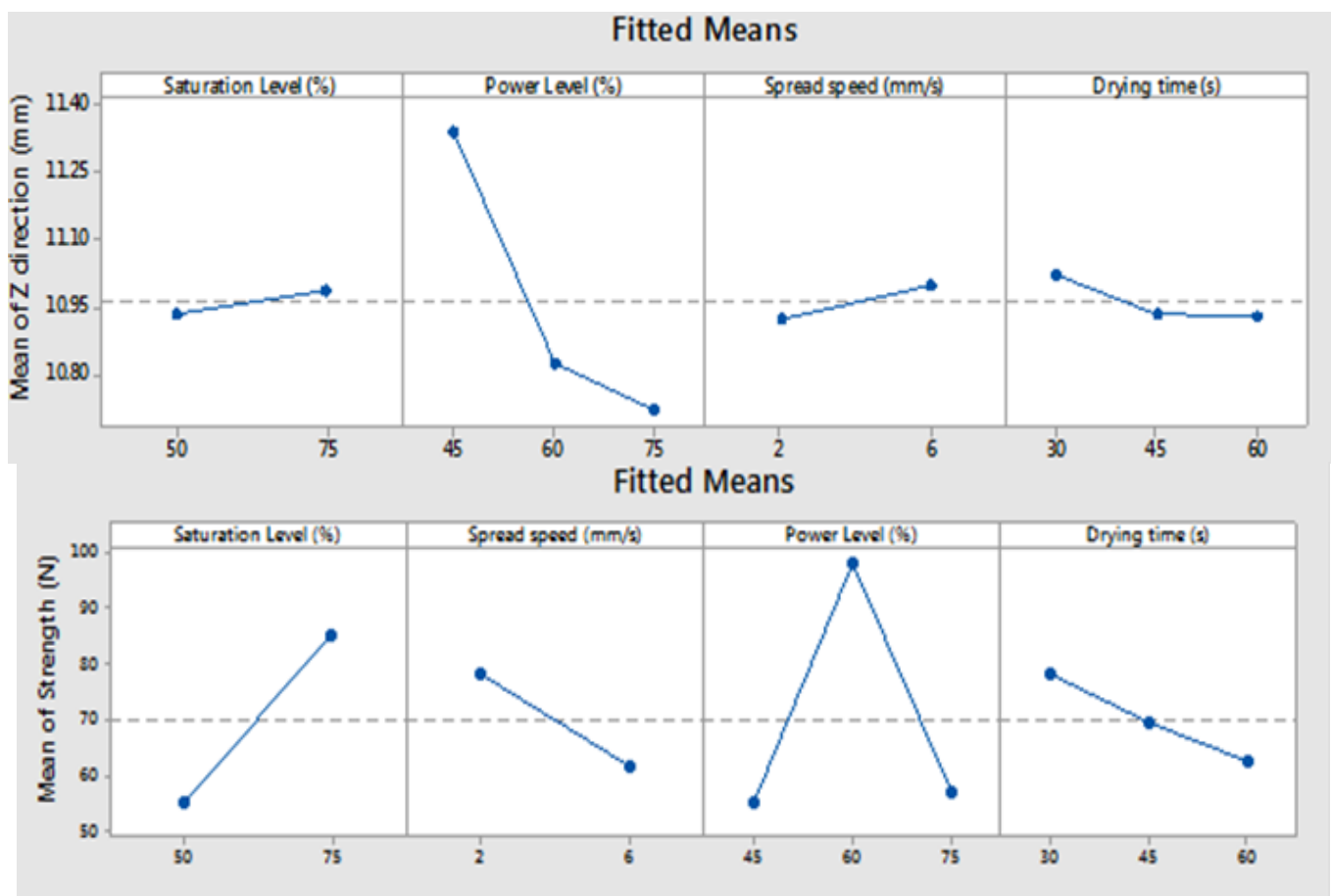

\section{Fig. 2.6 Effect of different factors on part accuracy in $\mathrm{z}$ direction and mechanical compressive strength [13]}

\subsubsection{In-process heating elements}

As it was explained previously, in Binder Jetting process the deposited binder for each layer needs to be partially cured before the subsequent layer of powder materials is spread. For this purpose, after the binder deposition the powder bed surface is subjected to set amount of heating applied via an external radiation heat source (e.g. heating lamp). The imposed heating reduces the binder solvent amount via evaporation and therefore reduces the total saturation level in the printed areas. This in-process heating has two elements, power level and drying time, which can usually be altered in the printer in order to reach the desired dryness of binder. Power level is defined as the intensity of applied heat using infrared heater and is set as a percentage of its maximum power by the operator, and drying 
time is the amount of time considered for drying (curing) of the deposited binder (i.e. the time the build platform stays under the heater).

In the literature, there exists very limited knowledge about the in-process heating effect on the green parts and final products after post-processing. In [14], it has been shown that at a constant saturation level of $50 \%$, although increasing power level from $55 \%$ to $65 \%$ significantly increases the mechanical strength of the green parts, this change does not seem to have apparent effects on the geometrical accuracies as it is shown in Fig. 2.7. This might be further explained by the relatively low saturation level and limited range of heating power $(55 \%-65 \%)$ chosen for printing the samples. Given these conditions, the binder could be adequately cured, and therefore no significant difference could be observed on the green parts dimensional accuracies.

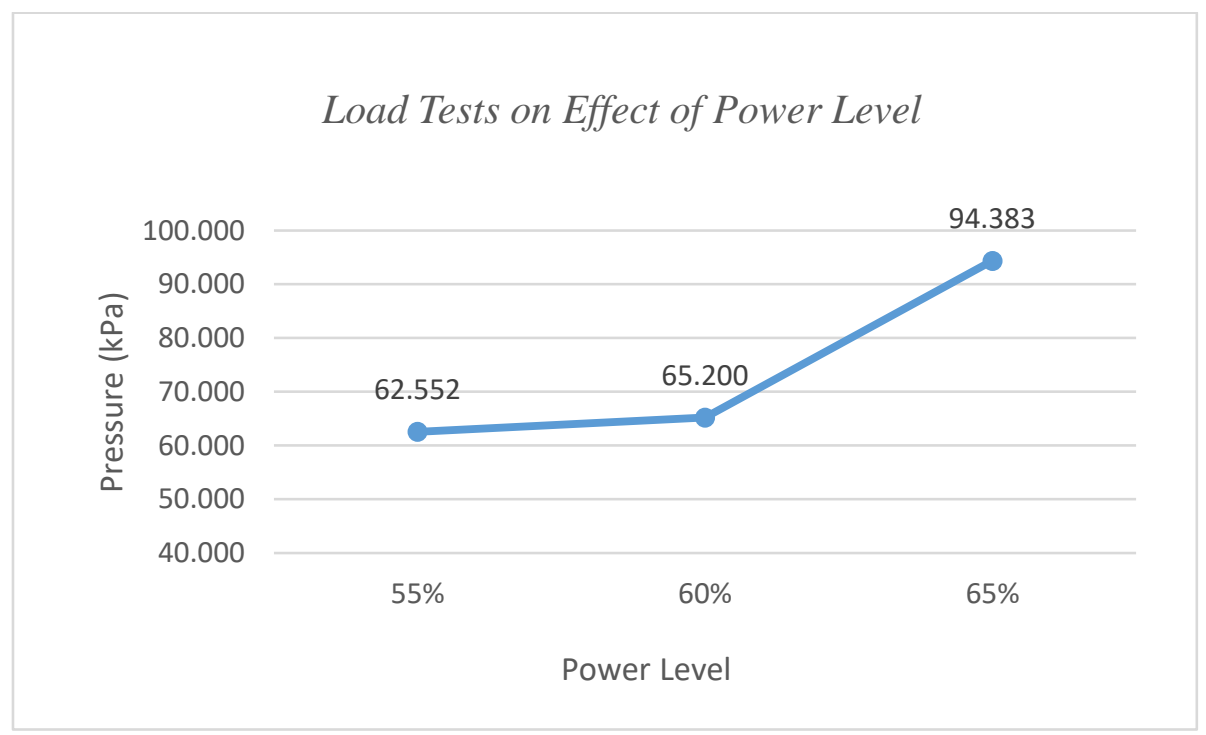

Fig. 2.7 Effect of power level on the compressive strength of the green parts [14]

Also, in the preliminary research conducted by the author the influence of inprocess heating on structural integrity of green parts was systematically investigated using full factorial experimental design. In Fig. 2.8, the compressive strength of green parts is 
displayed as a function in-process heating. From this figure, the power level and time for drying the binder should be selected in such a way that the sprayed binder is dried at an optimal condition. The higher the power level is set, the less the drying time would be required.

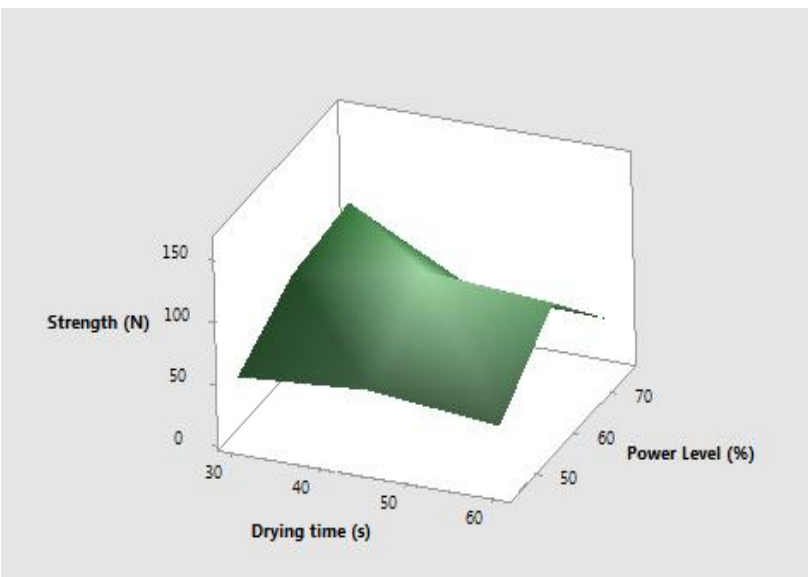

Fig. 2.8 Surface plot of green part strength vs drying time and power level

Observations indicated that mechanical strength and dimensional accuracy of green parts strongly depend on in-process heating elements which in turn determine the different curing conditions of the binder within the layers. As a conclusion, while excessive inprocess heating will prevent a strong bond to be created between successive layers by overcuring the binder, with insufficient in-process heating the under-cured binder within the layers won't have enough adhesion strength, which bonds the binder to powder particles, to retain the structural integrity as oppose to the shear forces involved in spreading new layers [13, 14]. 


\subsubsection{Layer thickness}

Layer thickness which is determined by the machine operator is used by the software to calculate the binder saturation amount according to equations (1-1) and (1-2). In these relationships, $\mathrm{V}_{\text {envelope }}$ is defined as

$$
\mathrm{V}_{\text {envelope }}=\mathrm{X} \text { spacing } * \mathrm{Y} \text { spacing } * \text { layer thickness }(\mathrm{Z} \text { spacing })
$$

Where $\mathrm{X}$ spacing and $\mathrm{Y}$ spacing are the distances between successive droplets in $\mathrm{X}$ and Y directions respectively (Fig. 2.2). It is clear from the relation that changing the layer thickness will directly affect the binder saturation amount and consequently the green part mechanical and dimensional characteristics. Moreover, the layer thickness information is utilized for processing and slicing the 3D digital model used for feature creation and therefore will influence the green part resolution. The greater layer thickness will result in lower resolution.

There exist multiple experimental-based studies in the literature that investigate the layer thickness, coupled with other process-related parameters such as binder saturation and part orientation, effect on green part mechanical and dimension properties. Lu et al [25] studied the fabrication of 3D mesh structures of $300 \mu \mathrm{m}$ wire width with TiNiHf SMA powder using Binder Jetting additive manufacturing process. SMA is referred to as the novel resilient materials which has the ability to return to the previously defined shape after being subjected to specified thermal or stress-induced procedures [31]. In this work, they showed how the dimensional accuracy varies as a function of layer thicknesses and binder saturation. Fig. 2.9 displays the printed wire width in terms of printing layer thickness and saturation [25]. They considered three scenarios for the interaction of binder saturation and 
the printing layer thickness as shown in Fig. 2.10. With a thin layer of the powder material, there exists more binder to flow laterally than vertically as the vertical diffusion of the binder is constrained with the previously saturated layer. This excessive lateral flow of the binder leads to larger and uneven wire width. When the printing layer thickness is too large, although uneven lateral spreading becomes less, there is more binder jetted into the powder bed because of the larger powder volume per layer. Therefore, due to larger volume of the binder, thicker wire width still results, with much less wire width variation. At the optimal printing layer thickness, binder vertical spreading and lateral spreading proceed and finish at approximately the same time. This very desirably leads to smallest wire width and the smallest wire width variation [25].

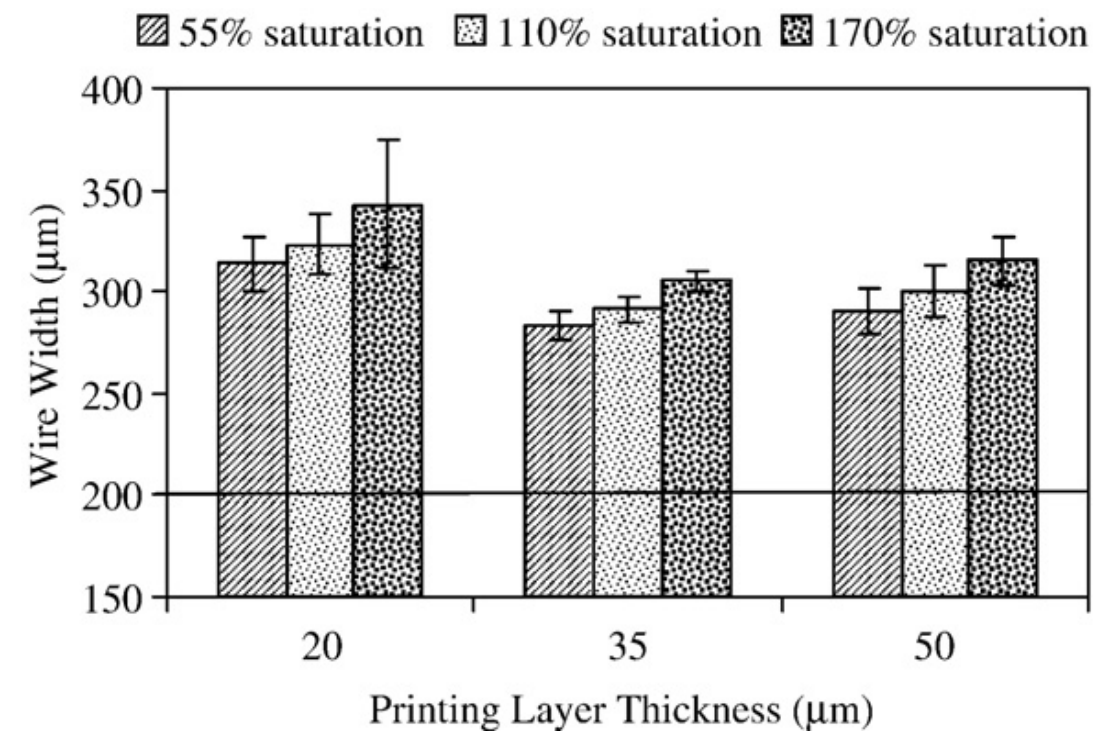

Fig. 2.9 Printed wire width measured by optical microscope [25]

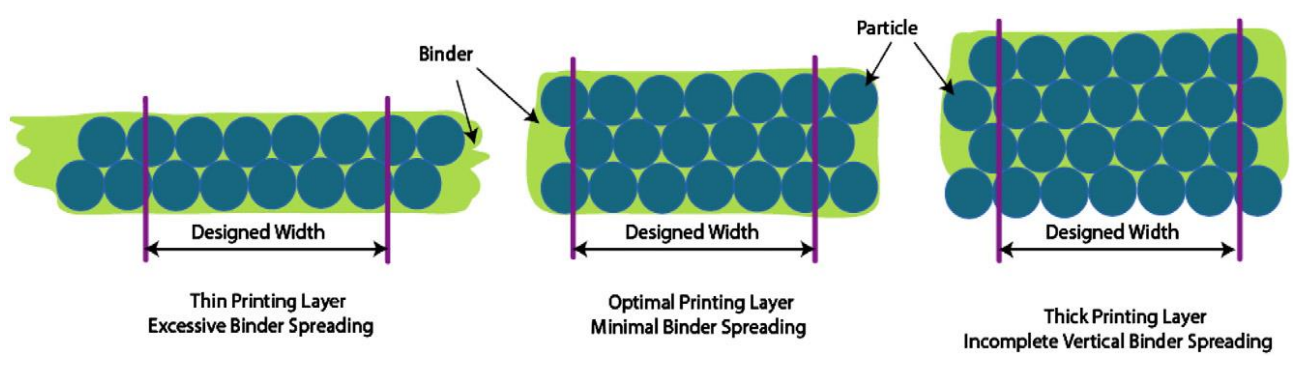

Fig. 2.10 Different printing layer thickness and binder spreading cases [25] 
The effects of the printing orientation and layer thickness on the physical and mechanical properties of plaster-based (zp150) scaffolds have been studied in [11]. In this research, it was concluded that while the layer thickness and printing direction have a significant effect on the scaffolds compressive strength of scaffolds as it is shown in Fig. 2.11, they have only a minor effect on the structural properties of scaffolds. The scaffolds printed in $\mathrm{X}$ and $\mathrm{Y}$ directions (Fig. 2.12) are sufficiently strong for handling and placement into a non-loading bone defects.

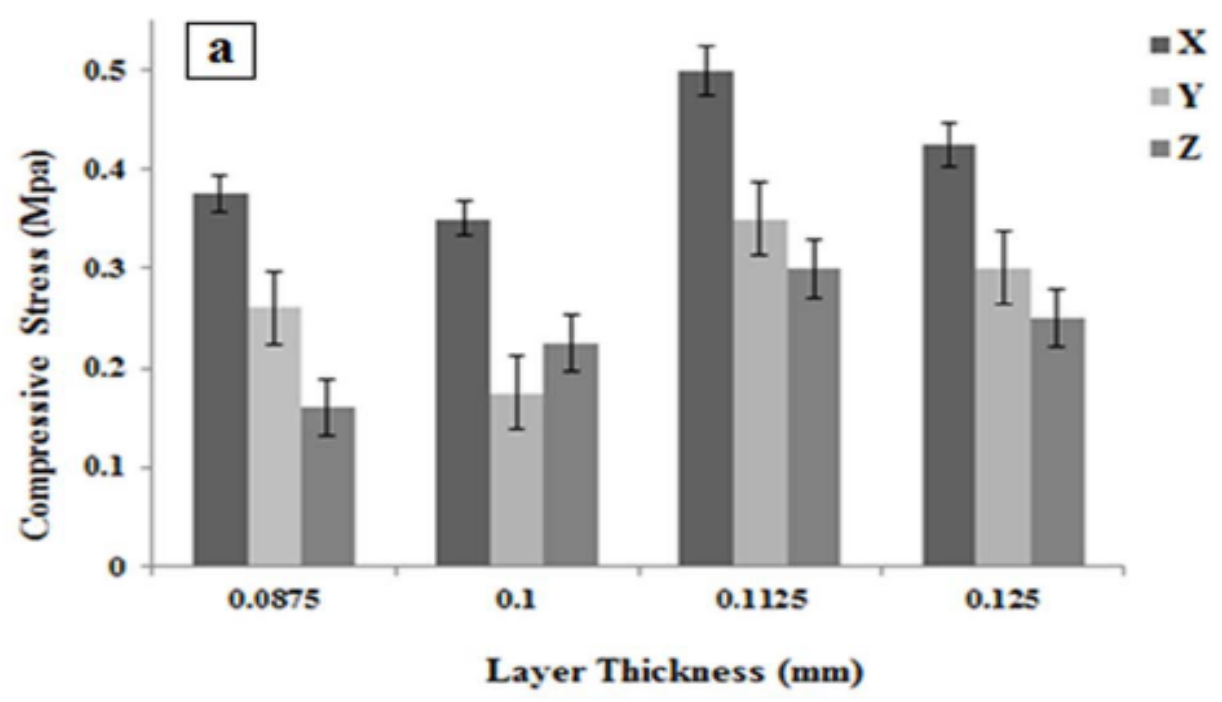

Fig. 2.11 Comparison of compressive strength in samples with different layer thickness in various orientations [11]

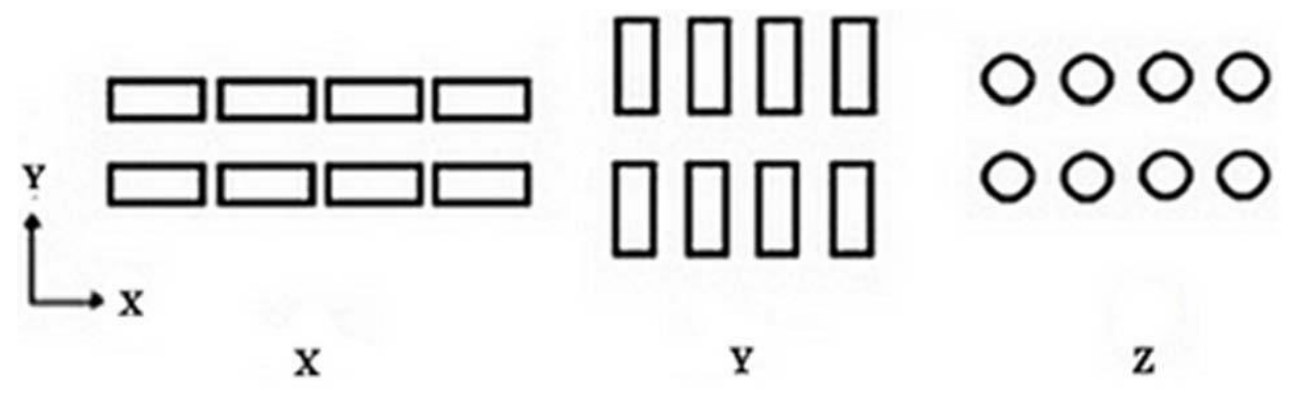

Fig. 2.12 Different printing orientation of samples [11] 


\subsection{2 material related parameters}

\subsubsection{Powder physical properties}

Particle size \& particle size distribution

Proper flowability is one of essential parameters in Binder Jetting additive manufacturing process to ensure the good quality of the green parts. Too low flowability reduces the printing resolution whereas too high flowability does not provide a powder bed sufficient stability required for 3DP [32].

It is well known that particle size directly influences flowability of powder material. Although fine particles tend to aggregate or/and agglomerate due to inter-particle forces, large particles usually flow more freely [33]. It is worth to be noted that agglomerates are defined as weakly bonded particles sticking together under van der Waals forces, whereas aggregates are considered to be constituted of particles which are bonded together by solid bridges [34]. In the literature there exist manifold examples of aggregated or agglomerated ceramic powders, depending on very diverse synthesis techniques, with submicron-sized primary particles [34-37]. Fig. 2.13 from [32] shows the dependency of flowability $\left(f f_{c}\right)$ on median particle size. These results reveal a linear correlation between the median particle size $\left(d_{50}\right)$ and flowability. A similar trend has been reported by Teunou [33] for flowabilty of powder materials with different particle sizes. 


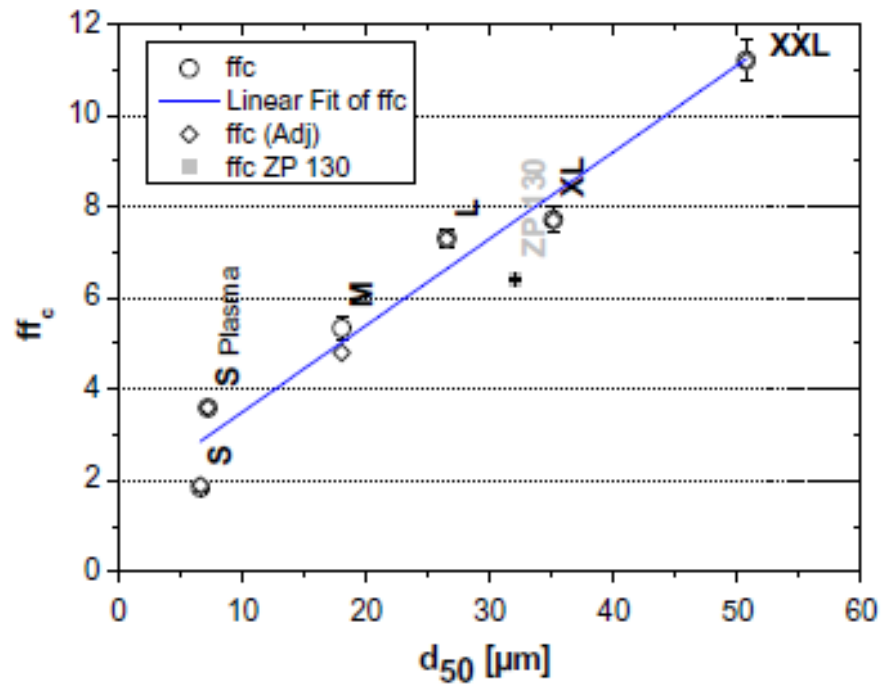

Fig. 2.13 Dependency of flowability (ffc) on median particle size [32]

In Binder Jetting process, high packing density of spread layers is always favorable in that it determines the subsequent mechanical properties, shrinkage, and density of final parts after sintering [38-40]. A high packing density depends directly upon the particle size distribution. There are many publications in the literature which have focused on the importance of the particle size distribution to obtain dense packing [41-46].

In [47], Streek has studied the effect of particle size on the packing density of tungsten powder as shown in Fig. 2.14. In general, the smaller the particle size leads to the lower density due to clumping or agglomeration effects. In practice, powders comprise a range of sizes, and the extent of the spread will also have a significant effect on the packing density. 


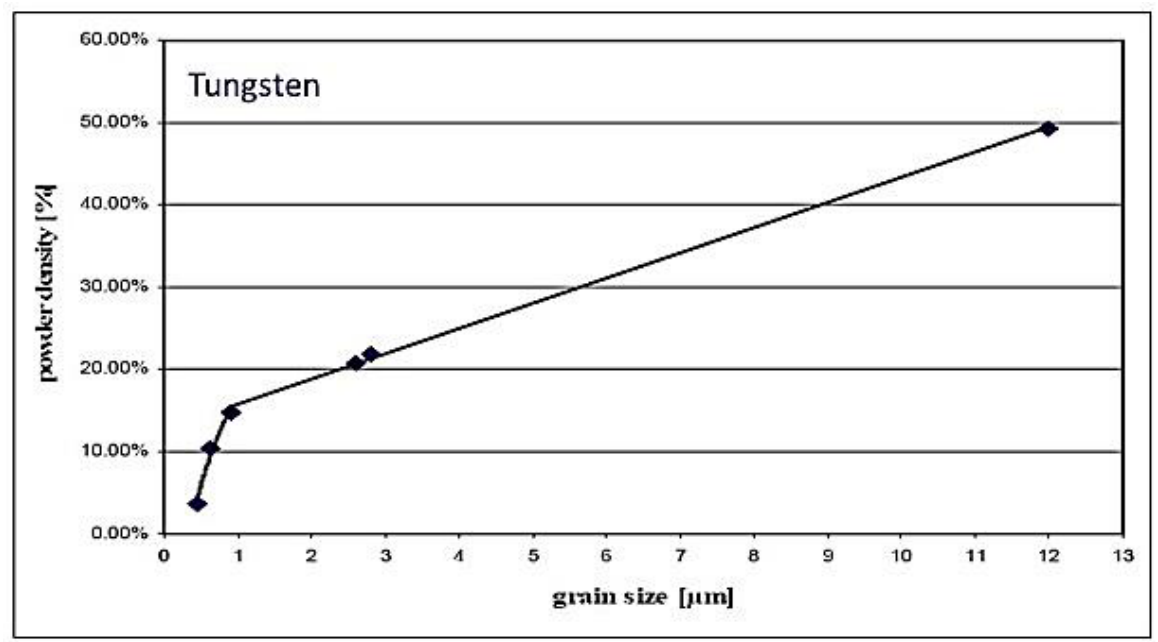

Fig. 2.14 The effect of particle (grain) size on packing density [47]

Karapakis [48] used bimodal powder blends (with varying combinations of coarse and fine particles) to determine how different ratios affected the layer density. Within the parameters of this study, it would appear that the best densities are obtained when the bimodal size difference is large, and the weight ratio of coarse to fine particles is between about 80:20 and 60:40.

For a two-component mixture of coarse and fine particles, the ideal packing density is predicted by Furnas model [41]. According to this model, the theoretical maximum packing efficiency $\mathrm{PE}_{\max }$ of a mixture of coarse and fine particles is calculated as [41]:

$$
P E_{\max }=P E_{c}+\left(1-P E_{c}\right) P E_{f}
$$

Or alternatively

$$
\mathrm{PE}_{\max }=1-\varphi_{\mathrm{c}} \varphi_{\mathrm{f}}
$$

Where $\mathrm{PE}_{\mathrm{c}}$ and $\mathrm{PE}_{\mathrm{f}}$ are the packing efficiency of the coarse and fine particles fractions, respectively, and $\varphi=1-\mathrm{PE}$, is the interstitial pore fraction of packed particles of a single size (fine or coarse particles only). 
Powder particle morphology

The morphology of the powder particles is of also great importance in determination of process efficiencies and the quality of the final products. Similar to powder particle size, morphologies need to be assessed and optimized as well. It is well known that the shape of powder particles influences the powder bed packing density, the degree of shrinkage during sintering, and also to some extent, the flowability of powder [49-51]. As an instance, the effect of particle shape on shrinkage of stainless steel is shown in Fig. 2.15. The stainless-steel material with irregular particle shapes demonstrates the highest shrinkage.

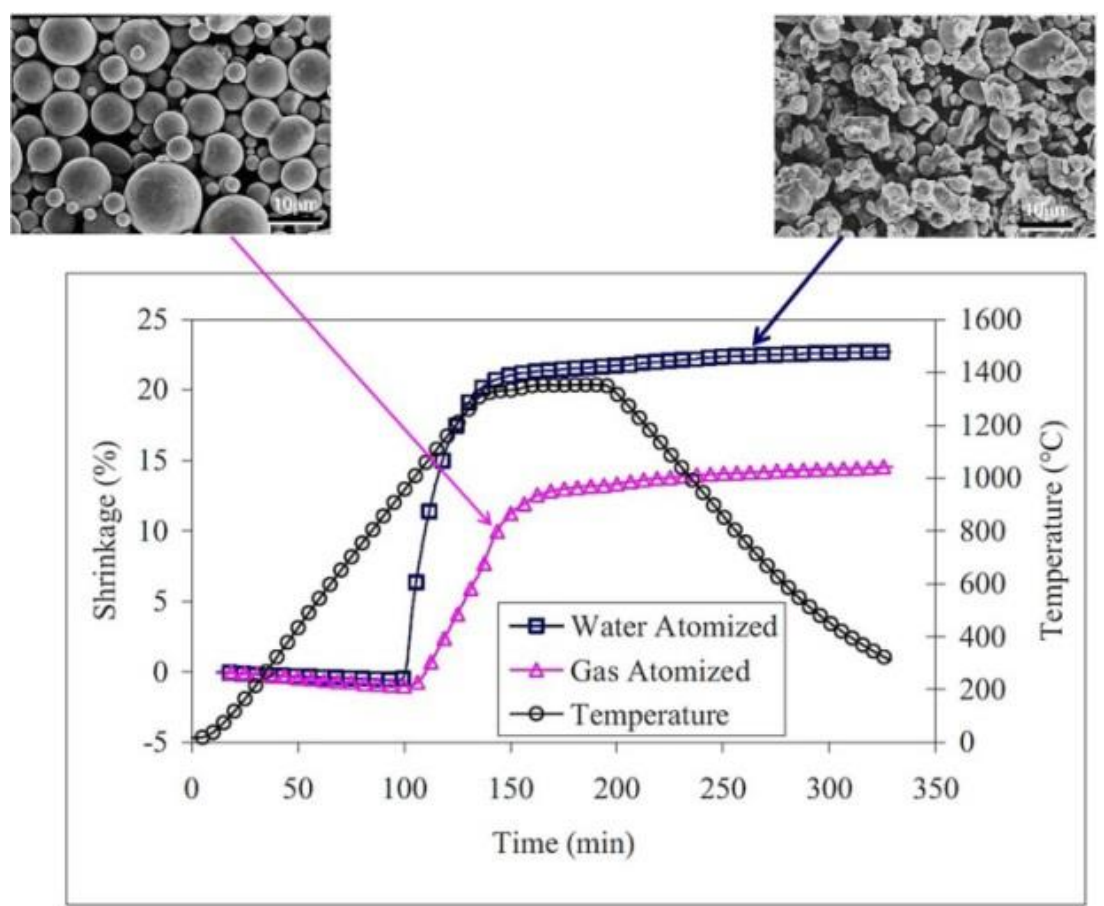

Fig. 2.15 The effect of particle shape on shrinkage of stainless steel during conventional furnace sintering [50]

\subsubsection{Binder physical properties}

Binder viscosity and surface tension 
In Binder Jetting additive manufacturing process, binder droplets are deposited on the surface of the powder bed via a multi-jet printhead. After impact, each droplet migrates into to powder bed due the capillary pressures (and to a lesser degree, gravitational forces) created between the liquid binder and powder bed particles. The migration of liquid binder in the powder bed will take place in all directions albeit with different lateral and vertical rates. Due to this difference in the migration rates, the liquid phase under equilibrium conditions would assume a specific profile depending on the physical properties of liquid binder including viscosity and surface tension, and interaction between the binder/powder bed [52-54]. The established profile characteristics have multiple implications to Binder Jetting process. Firstly, the permeation profile largely determines the minimum feature resolution of the fabrication process and consequently influence the geometrical accuracy. Secondly, the characteristic dimensions of the profile (i.e. lateral speeding distance and vertical permeation depth) impose restrictions to the selection of process parameters including layer thickness and in-process heating in order to achieve adequate part integrity. Thirdly, the liquid distribution within the powder bed influences the strength of the green parts in combination with the other process control parameters. Therefore, the binder physical characteristics are of great importance in determining the quality of the parts fabricated via Binder Jetting process.

\section{4 Feature Formation in Binder Jetting Process}

As it was explained earlier, the essence of feature fabrication in Binder Jetting process is to use liquid binder to join the powder particles in the designated areas of layered powder material. This technology uses DOD (drop-on-demand) printheads to deliver liquid 
binder droplets consecutively with certain lateral spacing. After complete penetration, each droplet assumes a profile (i.e. a mass of particles bonded by a single droplet) inside the powder bed. Bonding of these droplet profiles within a layer realizes a $2 \mathrm{D}$ slice of a 3D geometry. Stitching the 2D slices with sufficient bonding strength between successive layers forms the 3D component. Therefore, the droplet profile inside a powder bed as the smallest building element of any 3D printed component plays a key role in controlling the part quality and integrity. The characteristics of such profiles determines the lateral spacing between consecutive droplets, and as results, influences the dimensional accuracy and the strength of the fabricated parts $[14,55]$. Although there exists an abundance of the literature on modeling the interaction between a liquid droplet and a porous material, in the context of Binder Jetting process, very limited researches have been carried out in this regard. The following provides a general overview of studies on liquid-porous medium interaction modeling existed in the literature with special focus on the attempts most pertinent to Binder Jetting technology.

\subsubsection{Modeling of droplet-porous meduim interaction}

The droplet-porous material interaction can be categorized into static and dynamic phases. While the static phase refers to the state where the penetration of the liquid droplet into the porous medium is complete, during dynamic phase of the interaction the droplet migrates into the porous material under the different driving phases. In Binder Jetting process, both phases are of crucial importance in controlling the part quality. The dropletporous medium interaction in the static phase determines the optimal saturation level 
required for successful printing whereas the interaction dynamic phase controls the profile of the area that is saturated by the droplet.

2.4.1.1 Droplet-porous miduim interaction at equilibrium (equilibrium saturation)

In Binder Jetting Technology, once the binder droplets are deposited on the selective regions, the liquid binder will migrate into the powder through pores located on the surface of the powder bed (so called imbibition) due to driving mechanisms such capillary attraction and gravity $[13,52,56,57]$. After imbibition is complete (i.e. after all the liquid binder permeates into porous medium), the drainage in which liquid binder migrates from the completely saturated region into the dry powder surrounding it, will begin to take place. Therefore, as binder migration progresses, the saturated regions drain and the dry regions imbibe until driving forces of both regions become equal. This is the state where equilibrium condition in which the driving forces are equal for both imbibition and drainage, is reached. Fig. 2.16 displays schematic binder and powder bed interaction sequence.

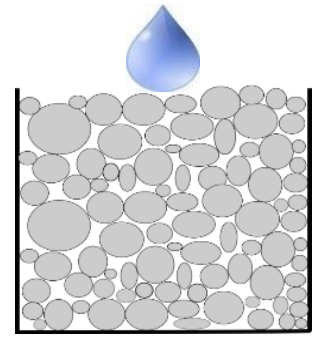

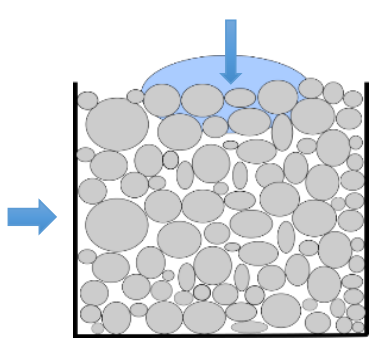

Imbibition

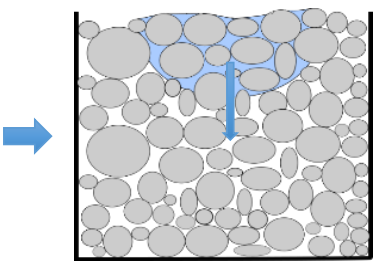

Drainage



Equilibrium

Fig. 2.16 Schematic diagram of binder migration in the powder bed

The saturation level (the ratio of amount of liquid binder to the pore volume in the print material) in the equilibrium phase is greatly important in BJ-AM process. For printing 
purpose, in practice the equilibrium saturation must be assumed as the saturation level which is specified by the machine settings. If the equilibrium saturation is much smaller than the saturation imposed by machine control parameters, the excess liquid binder will tend to migrate out of the designed boundaries of the part. On the other hand, If the equilibrium saturation is much greater than the machine-imposed saturation, the printed part won't have sufficient mechanical strength due to lack of enough amount of binder, which will consequently result in weak bound between the particles and also successive layers. It has been experimentally shown that binder amount plays a major a role in controlling the part accuracy and strength $[25,26,58]$. Therefore, it is quite beneficial to be able to predict the equilibrium saturation for a given powder bed and liquid binder using physics-based modeling before the printing process.

One of the few attempts in this regard was reported by J. F. Bredt. In his PhD dissertation conducted at MIT, a model to predict the equilibrium fluid content of printed features from measurements of the capillary characteristics of the powder and physical properties of the fluid binder was developed [24]. It is proposed in this model that a balance of capillary pressure exists between a partially saturated region and the dry powder surrounding the feature. In this Model it is assumed that the interaction between binder and powder in BJ-AM process is dominated by capillary, and to lesser degree, gravitational forces. The liquid binder in the powder bed distributes itself in the pores and contacts the powder surface with a local contact angle of $\theta$ determined by the surface energies of the solid, liquid, and vapor phase boundaries (Fig. 2.17). 


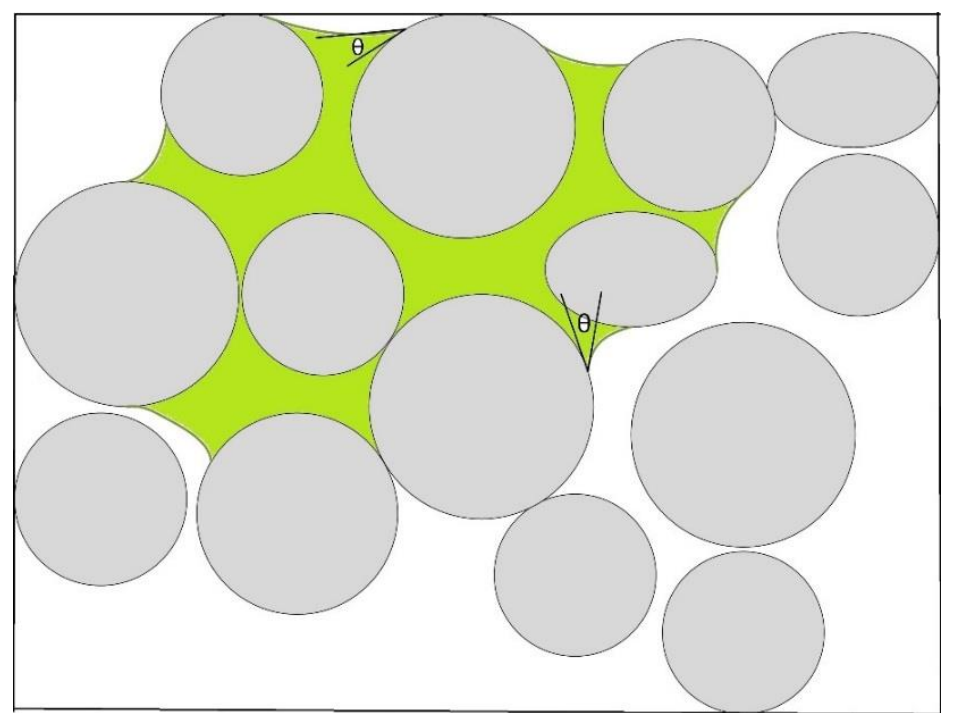

Fig. 2.17 Local contact angle between liquid binder and a particle in the powder bed

Although the meniscus formed in the vapor-liquid interface may assume a complex profile, it possesses a mean curvature and capillary pressure, defined uniquely everywhere in the binder. On the basis of the Laplace equation, capillary pressure for a local curved interface (local curvature) within a pore is presented by [59]:

$$
\Delta p=p_{c}=\gamma\left(\frac{1}{r_{1}}+\frac{1}{r_{2}}\right)
$$

where $\Delta \mathrm{p}$ is the capillary pressure across the fluid interface, $\gamma$ is the surface tension is the $\mathrm{r} 1$ and $\mathrm{r} 2$ are the principal radii of curvature. For a capillary tube of radius $\mathrm{r}$, the equation (1-6) becomes as follows [59]:

$$
p_{c}=\frac{2 \gamma \cos \theta}{r}
$$

For a real porous material where a liquid binder and powder interact, the outcome of the above equation would be a statistical average which is obtained over the void spaces in the vicinity of the considered pore [60]. From the equation, capillary pressure depends on the geometry of pore, contact angle $\theta$, and the degree of saturation (Sw). On the other hand, since the relationship of capillary pressure on saturation level $(\mathrm{pc}=\mathrm{pc}(\mathrm{Sw}))$ cannot 
be analytically described due to the shape irregularity and complexity of pores in an actual porous material, laboratory experiments could be implemented to derive the relationship for any given porous media. A typical curve of capillary pressure as a function of saturation is shown in Fig. 2.18.

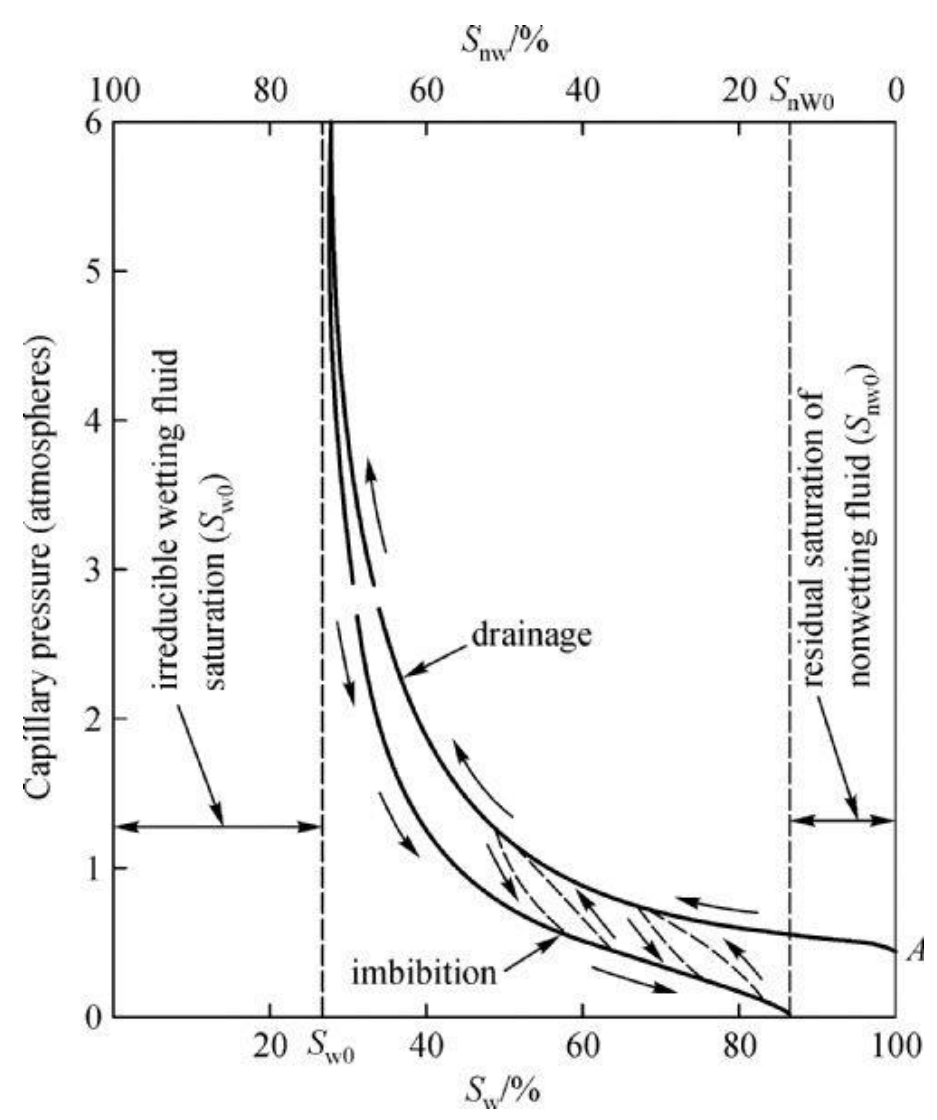

Fig. 2.18 Typical capillary pressure-wetting fluid saturation curves [24]

From the figure, the curve follows two different paths for imbibition and drainage illustrating hysteresis. The hysteresis in the capillary pressure are known to be rendered by two mechanisms, contact angle $(\theta)$ and geometry of void space. While the former depends on the direction of the displacement, the latter arises from many bottlenecks of pores in the powder bed (ink-bottle effect) [60]. Due to the hysteresis in the capillary pressure curve, the saturated region and dry region the feature can exist at equilibrium with different fluid contents since each of these regions has different pressure characteristics. 
There are two important features in Fig. 2.18. Irreducible wetting saturation, Swo, is a critical wetting fluid saturation at which the fluid displacement in the porous medium approaches zero. The capillary pressure at this saturation is called the dry suction pressure P0 which is a critical characteristic of a porous medium in determining the liquid binder migration during feature formation in BJ-AM process. The second point in Fig. 2.18 labelled $\mathrm{A}$ on the right side of the graph is the minimum pressure (also called bubbling pressure, PB) required to initiate the displacement of a wetting fluid (e.g. liquid binder) in a fully saturated porous medium.

In the model developed in [24], capillary pressure has been considered as the only driving force for binder migration, and equation (1-7) was used as the governing relationship between the capillary pressure and liquid binder saturation. In this model, pore curvature $(\mathrm{K})$ is defined in such a way that term $\mathrm{r}$ is eliminated from the equation [24]:

$$
p_{c}=\frac{-2 \gamma_{L V} \cos \theta}{r}=-K \gamma_{L V} \cos \theta
$$

Hysteresis in the capillary pressure between imbibition and drainage of a fluid results in two pore curvature for a single pore and for an assemblage of pores. The critical nonwetting curvature, $\mathrm{K}_{\mathrm{NW}}$, is defined by the bubble pressure, $\mathrm{PB}$, and critical wetting curvature $\mathrm{KW}$ is defined by $\mathrm{P} 0$ as follows [24]:

$$
\begin{aligned}
& K_{W}=\frac{2}{R_{W}}=\frac{-P_{0}}{\gamma_{L V} \cos \theta_{a d v}} \\
& K_{N W}=\frac{2}{R_{N W}}=\frac{-P_{B}}{\gamma_{L V} \cos \theta_{r e c}}
\end{aligned}
$$

where $\theta_{a d v}$ is advancing contact angle, and $\theta_{r e c}$ is the receding contact angle. 
The quantities $R_{W}$ and $R_{N W}$ describe the pore geometry of the powder bed. There two radii are the dimensions of analogous cylindrical capillaries exerting the same capillary pressures as the dry powder and the saturated region, respectively. In Fredric's model, a fully saturated feature is modelled as a cavity with two capillaries, one with radius $R_{W}$ that represents the path of dry pores that lead out into bulk powder bed, and the other one with a radius $R_{N W}$ that represents the trail of the saturated pores that guide air into the feature as it is shown in Fig. 2.19.

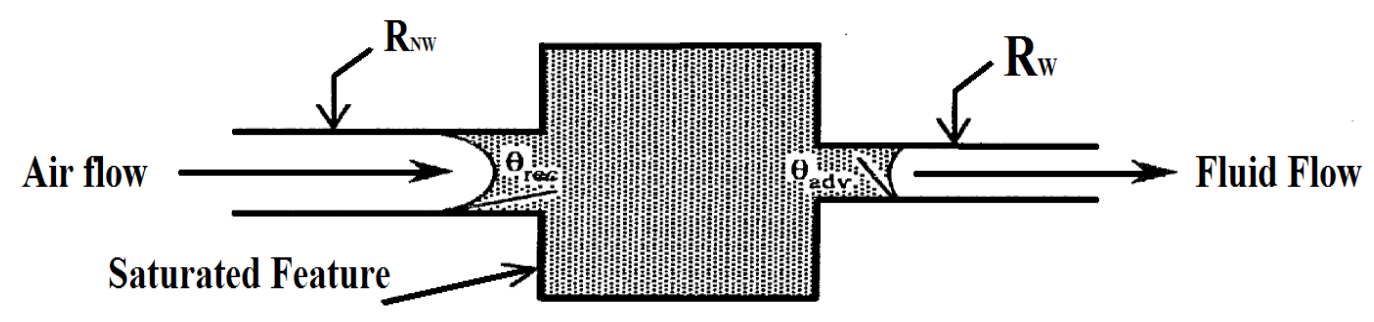

Fig. 2.19 The model for equilibrium of a liquid binder in a hydraulic feature [24]

Liquid pressure can be transmitted across this feature. Besides fully saturated regions, pores that have been partially drained but still in hydraulic contact are included in this model.

If the pressure within the larger capillary $\left(\mathrm{R}_{\mathrm{NW}}\right)$ is greater than that of the smaller capillary $\left(\mathrm{R}_{\mathrm{W}}\right)$ in Fig. 2.19, fluid will spontaneously migrate from left to right. In the current model, the excess pressure in the feature is expressed in terms of the differences in the pressure between two capillaries [24]:

$$
\Delta P=-P_{0}+P_{B}
$$

In terms of the critical radii becomes

$$
\Delta P=2 \gamma_{L V}\left[\frac{\cos \theta_{a d v}}{R_{W}}-\frac{\cos \theta_{r e c}}{R_{N W}}\right]
$$


Or alternatively,

$$
\Delta P=\gamma_{L V}\left[K_{W} \cos \theta_{a d v}-K_{N W} \cos \theta_{r e c}\right]
$$

This excess pressure is driving force for fluid migration in a porous medium. Liquid binder which is often employed in printing process is highly wetting to the powder (i.e. $\left.\cos \theta_{a d v} \approx \cos \theta_{\text {rec }} \approx 1\right)$. Under these circumstances, the difference in critical pore curvatures $\left(K_{W}-K_{N W}\right)$ defines the driving force for binder permeation out of a fully saturated region. As a feature becomes less than fully saturated, the excess capillary pressure is proportional to the difference $\left[K_{W} \cos \theta_{a d v}-K_{\text {drainage }} \cos \theta_{\text {rec }}\right]$ where $K_{\text {drainage }}$ is dependent on the saturation. Therefore, the condition for hydrostatic equilibrium of a feature is

$$
K_{\text {drainage }}\left(S^{*}\right)=K_{W}
$$

where $\mathrm{S}^{*}$ is the equilibrium saturation.

Therefore, given the powder bed and liquid binder characteristic such as the suction pressure, the capillary pressure curves, surface tension, contact angle, etc. it is possible to estimate the equilibrium saturation of a printed feature using the relationship by Eq. (1-14). However, our preliminary work revealed that there exists a discrepancy between the estimate obtained from the model and the equilibrium saturation which was measured from printed samples using M-Lab ExOne machine. Fig. 2.20 displays the printed features used for determining the saturation in the equilibrium state. It was found out that the discrepancy is about $30 \%$. 



Fig. 2.20 Two examples of printed features for empirical equilibrium saturation measurements

The contributing factors to this discrepancy might be summarized as follows.

In the current model, capillary pressure/saturation curve in drainage is used to determine the final saturation amount using the dry suction pressure which will always lead to higher saturation level. In the equilibrium state there exists a trail of partially saturated pore with an irreducible wetting saturation level where the liquid binder ceases to migrate. The simplest case of this phenomenon is shown in Fig. 2.21 with only two pores. In this picture, pore 1 drains and pore 2 imbibe until the capillary pressures between two pores become equal. The capillary pressure corresponding to pore 2 is referred to as irreducible wetting saturation. Due to the capillary pressure hysteresis between drainage and imbibition, if the capillary pressure characteristic curve in drainage is used for predicting the equilibrium saturation (which is the case in the model), this estimate will always be higher than actual saturation.

The other factor which might be contributing to higher estimate of equilibrium saturation is neglecting gravity effect particularly in the vertical direction. The effect of gravity on the hydrostatic pressure within a feature can cause fluid to flow further downward out of the boundaries. Consideration of gravity effect in the model will result in 
higher driving pressures for binder migration which in turn lead to lower estimate of the saturation in the equilibrium condition.

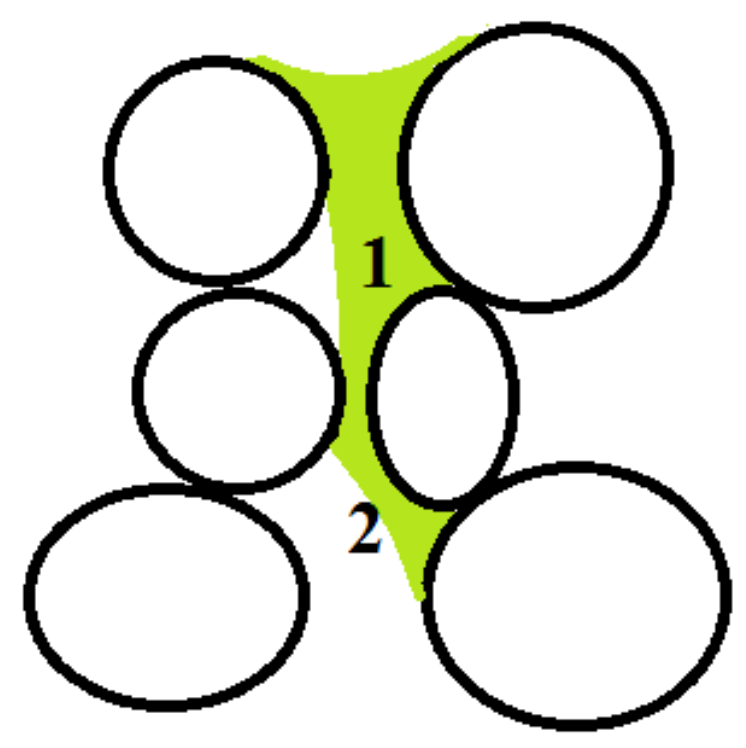

Fig. 2.21 Equilibrium state of two partially saturated pores

Furthermore, classic theories for the fluid permeation in porous media has been extensively investigated in the context of liquid (commonly water)-soil interactions for various civil engineering applications. In this area, there exist manifold mechanisms including capillary force, viscous friction, gravity and osmosis which are assumed to play significant roles in liquid migration in a porous medium. Among these mechanisms, viscous friction is considered to be more relevant during the dynamic permeation advance process and the determination of the permeation time but in general not critical to the permeation equilibrium [61, 62]. Additionally, osmosis phenomenon is more pronounced in soils and clays there is chemical composition gradients between the phases [63]. Therefore, it is not expected to be a significant contributing parameter for the Binder Jetting process. 
The contribution of gravity to the permeation process could be manifested in two forms: the droplet inertia resulted from the initial droplet impact and the downward gravitational pulling during the permeation. Although previous works have suggested that the gravitational pulling effect could be neglected during the permeation process when the droplet size is sufficiently low, more research needs to be conducted to evaluate the effect of gravity on binder-powder bed interaction in BJ-AM systems where powder particles are free to displace under even small forces such as inertial forces.

\subsubsection{Droplet-porous miduim interaction (dynamic phase)}

The study of the dynamics of capillary penetration was initiated by Lucas [64] and Washburn [57]. Their theoretical model assumed a fully developed, quasi-steady-state laminar flow of a Newtonian liquid, an infinite reservoir, negligible inertia effects, negligible fluid resistance, a constant radius, and a constant contact angle. Based upon these assumptions, the following two equations were developed for the rate of penetration of a liquid into an empty capillary, in the absence or in the presence of a gravitational field, respectively:

$$
\begin{gathered}
\mathrm{X}^{2}=2 \tau \\
-\frac{X}{G}-\frac{\ln (1-G X)}{G^{2}}=\tau
\end{gathered}
$$

The dimensionless penetration distance, $X$, dimensionless time, $\tau$, and dimensionless gravitational coefficient, G, are defined by

$$
\begin{array}{r}
X=\frac{1}{r} \\
\tau=\frac{2 \gamma|\cos \Theta|}{8 \mu r} \mathrm{t}
\end{array}
$$


and

$$
\mathrm{G}=\frac{\rho g r^{2} \sin \alpha}{2 \gamma|\cos \theta|}
$$

In these equations, 1 is the length of the liquid in the capillary; $r$ is the radius of the capillary; $\gamma, \mu$, and $\rho$ are the surface tension, viscosity, and density of the liquid, respectively, $\mathrm{t}$ is the time, $\mathrm{g}$ is the gravitational acceleration, and $\mathrm{a}$ is the inclination between the capillary and the horizontal as it is shown in Fig. 2.22.

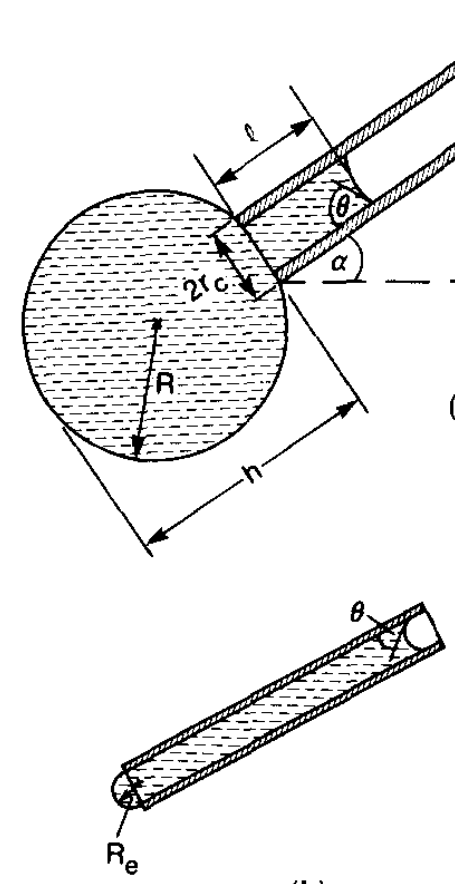

(b)

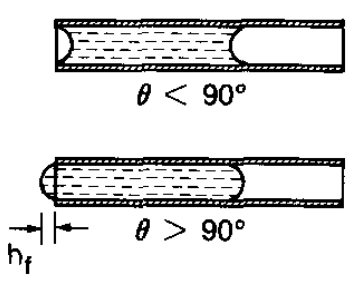

(c)

Fig. 2.22 The definition of the system. (b) The general equilibrium situation. (c) Final equilibrium situation in the absence of gravity, for $\theta<90^{\circ}$ and for $\theta>90^{\circ}$ [57]

Following Lucas and Washburn pioneering studies [52, 64], research on the theory of the capillary penetration rate has diversified to account for the factors neglected in Eq. (1-15) and (1-16). Two main directions of research that have been followed include resolving the hydrodynamic complications which are related to the bulk flow of the liquid [65-70], and the focusing on problems associated with the contact angle and the motion of 
the solid-liquid fluid contact line [71-78]. In addition, the theory of capillary penetration has been extended to non-uniform capillaries and porous media [79-81] and to pre-wet capillaries [82].

The basic assumption of the Lacus-Washburn equations, the infinite size of the liquid reservoir, has been tackled in [57]. Marmur has regarded the thermodynamics and kinetics of a finite small drop which is brought into contact with a capillary [57]. In this work he concludes that the effect of the drop size stems from the increased pressure inside the drop due to its curvature.

In Marmur's study, the basic differential equation stated in Washburn's work [52, 64] are then rewritten in terms of the dimensionless quantities as follows.

$$
X \frac{d X}{d \tau}=(U+Y-G X)
$$

Where

$$
\begin{aligned}
& \mathrm{U}=\frac{\cos \theta}{|\cos \theta|} \\
& \mathrm{Y}=\frac{r}{R|\cos \theta|}
\end{aligned}
$$

Since Eq. (1-20) involves the instantaneous radius of the drop through Y, an additional equation is required to elucidate this radius. This equation stems from the conservation of the volume of the liquid, and can be written, neglecting the curvature of the meniscus, as

$$
\mathrm{V}=\mathrm{V} 0-3 \mathrm{X}
$$

where the dimensionless volume, $\mathrm{V}$, is related to the true volume, $\vartheta$, by 


$$
\mathrm{V}=\frac{3 \vartheta}{\pi r^{3}}
$$

and the dimensionless initial volume of the drop, V0, is defined in the same manner as $\mathrm{V}$. The instantaneous radius can then be calculated, using simple geometrical considerations, from

$$
\rho=\frac{V}{3 H^{2}}+\frac{H}{3}
$$

Where

$$
\rho \equiv \frac{R}{r}
$$

And

$$
\mathrm{H} \equiv \frac{h}{r}
$$

After taking into account all the above parameters, the following equation is concluded for the rate of the penetration of a droplet with a finite size:

$$
\frac{B}{A^{2}} \ln \frac{B}{A X+B}+\frac{X}{A} \cong \tau
$$

Where

$$
\begin{aligned}
& \mathrm{A} \equiv 1.5874 V_{0}^{-\frac{4}{3}}\left|(\cos \theta)^{-1}\right|-G \\
& \mathrm{~B} \equiv U+1.5874 V_{0}^{-\frac{1}{3}}\left|(\cos \theta)^{-1}\right|
\end{aligned}
$$

Marmur has further investigated the fluid radial penetration between two flat plates in an attempt to model the spread of a liquid in a thin layer of porous medium. Despite its simplicity, the radial capillary model mimics some of the essential features of penetration into a thin porous medium, and can serve as a limiting model for penetration into thin 
porous media [83]. This model system is described in Fig. 2.23. The radial capillary consists of two parallel, rigid and nonporous plates, the distance between which is d. A small hole exists in one of the plates, through which the liquid may penetrate. The effect of gravity is neglected in the analysis.

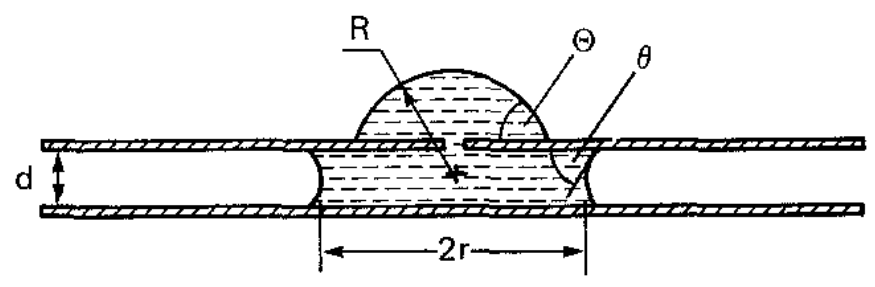

(a)

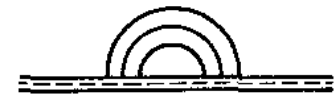

(b)

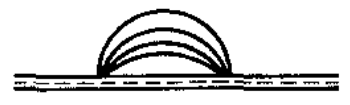

(c)

Fig. 2.23 The radial capillary. (b) Penetration without hysteresis. (c) Penetration with a constant basal radius [84]

For the radial penetration of a fluid between the flat plates, the following equations have been suggested in this study [83].

$$
y \ln \left(\frac{y}{y_{o}}\right) d y=\left(U-\frac{1}{2 y|\cos \theta|}+\frac{1}{\rho|\cos \theta|}\right) d \tau_{p}
$$

Where

$$
\begin{array}{r}
\mathrm{Y} \equiv \frac{r}{d}, y_{0}=\frac{r_{0}}{d} \\
\mathrm{U}=\frac{\cos \theta}{|\cos \theta|}, \rho \equiv \frac{R}{d}, \tau_{p} \equiv \frac{\gamma|\cos \theta|}{6 \mu d} t
\end{array}
$$

During the penetration period, change of the drop radius greatly affects the rate of penetration. The real behavior of the drop, which may be complex because of contact angle hysteresis, can be bound between two limiting situations: (a) no hysteresis, where the 
contact angle between the drop and the outside surface of the plate remains constant, and (b) constant basal radius with a decreasing contact angle.

No hysteresis

The constancy of the volume of the liquid, which yields the value of $\rho$ required in Eq. (1-31), is expressed by dimensionless variables as

$$
\rho=\left[\frac{4 \rho_{0}^{3}-3 y^{2}}{(1-\cos \theta)^{2}(2+\cos \theta)}\right]^{1 / 3}
$$

where the dimensionless radius of the drop prior to contacting the plate, $\rho_{0}$, is related to the initial radius of the drop (prior to contacting the plate), $\mathrm{R}_{0}$, by

$$
\rho_{0} \equiv \frac{R_{0}}{d}
$$

Constant basal radius

Eq. (1-34) for the constancy of the volume of the liquid remains valid; however, the value of $\theta$ now varies. Therefore, Eq. (1-34) has to be solved simultaneously with the following equation, which relates $\rho$ with the constant dimensionless radius of the base of the drop, $\mathrm{y}_{\mathrm{b}}$ :

$$
\rho=\frac{y_{b}}{\operatorname{Sin} \theta}
$$

Where

$$
y_{b}^{3}=4 \rho_{0}^{3} \frac{\sin ^{3} \theta_{0}}{\left(1-\cos \theta_{0}\right)^{2}\left(2+\cos \theta_{0}\right)}
$$

In the case of infinite reservoir, Eq. (1-31) turns into:

$$
y \ln \left(\frac{y}{y_{0}}\right) d y=\left(1-\frac{1}{2 y \cos \theta}\right) d \tau_{p}
$$


The replacement of $U$ by 1 and the removal of the absolute magnitude brackets from $\cos \theta$ emphasize that penetration is possible in this case only for $\theta<90^{\circ}$. This is so because the pressure in the infinite liquid reservoir equals the ambient pressure and does not offer an additional driving force as in the case of a small drop. This system suggested by Marmur may be useful in understanding the geometrical effect related to a radially expanding liquid front on the process of capillary penetration.

Denesuk et al. [53] considered the penetration of a finite size, liquid droplet into a porous body modelled as cylindrical pores distributed uniformly over the surface. An integral equation describing the depletion process for the general case of arbitrary contact radius behavior is derived and solved for the limiting cases of constant contact angle and of the constant position of the contact line. This work does not include the impact of the gravity or of the pressure internal to the droplet. The neglect of the gravity is assumed to be valid for essentially all porous media, and the neglect of the pressure internal to the droplet is claimed to be valid for the droplets which are not externally small. For example, for a spherical cap droplet with a contact angle of 20 and contact radius of about $0.7 \mathrm{~mm}$, the excess pressure inside the drop is on the order of only $10^{-4}$ of an atmosphere. The impact of gravity on the shape of the droplet should act only to diminish the excess pressure [53].

In Denesuk's model, the porous material is initially modeled as a solid with an array of parallel, right cylindrical pores as it is schematically shown in Fig. 2.24. The cylindrical pores are assumed to be of infinite depth, of some averaged or effective constant radius, $\mathrm{R}$, and of some number density/ unit area, $\rho_{P}$. In this study, the following equation is presented for calculating the rate of liquid spreading $r_{s c}(t)$. 


$$
r_{S C}^{3}(t)=\frac{V_{0}}{\xi(\theta)}-\frac{K}{2 \xi(\theta)} \int_{0}^{t} \frac{r_{d}^{2}\left(t^{\prime}\right)}{\sqrt{t^{\prime}}} d t^{\prime}
$$

In this formulation, $\mathrm{V}_{0}$ is the total liquid volume, $\mathrm{K}$ is the permeability, $\mathrm{r}_{\mathrm{d}}$ (the drawing radius) is the radius associated with the effective drawing area of the droplet (Fig. $2.25), \mathrm{r}_{\mathrm{sc}}$ (the apparent contact radius) is the contact radius associated with the spherical cap, and

$$
\xi(\theta)=\frac{\pi}{3} \frac{(1-\cos \theta)(2+\cos \theta)}{\sin \theta(1+\cos \theta)}
$$
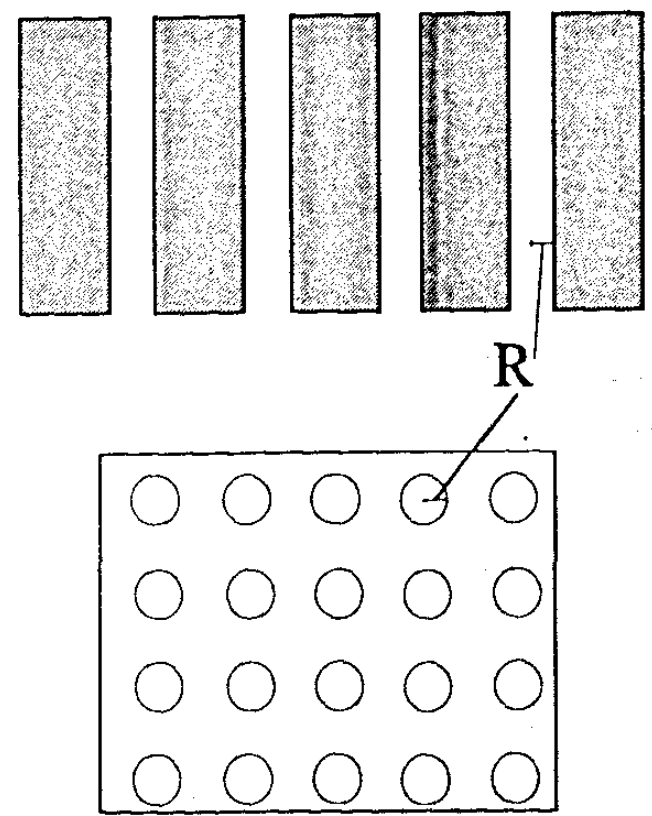

Fig. 2.24 Cross section (upper) and top view (lower) of the geometry for the model of the porous surface (schematic) [85] 


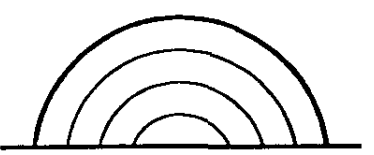

(a)

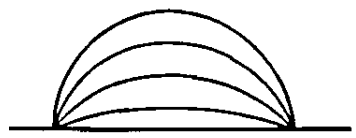

(1)

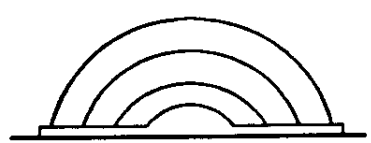

(2)

(b)

Fig. 2.25 (a) Schematic representation of the decreasing drawing area (b) the constant drawing area (the thickness of the remnant film in case $b$ (2) is exaggerated for illustrative purpose). In (a), rsc $=r d$, both decrease with time; in b (1), rsc = rd = constant; and in $b(2)$ rsc decreases with time and $\mathbf{r d}=$ constant $[85]$

In order to obtain simple expressions for the time dependent $r_{s c}$, limiting cases, Constant Drawing Area (CDA) and Decreasing Drawing Area (DDA), with Fixed Contact Angle have been considered as it has been illustrated in Fig. 2.25.

Constant Drawing Area (CDA)

If the area of liquid in contact with the porous solid remains constant throughout the liquid depletion process the apparent drawing radius $r_{d}$ may be assumed constant, and the resulting model will represent the lower limit on the time necessary to deplete a given droplet volume.

This limiting case may represent two different physical situations: a fixed contact line position where the contact angle decreases as the droplet volume is depleted (Fig. 2.25 b-1); the apparent contact line which recedes over a layer of liquid, where the position of the actual contact line of the layer remains fixed, and the top layer is thick enough to act as 
an unimpending path for liquid to flow from the droplet to pores. This thin liquid layer is referred to as remnant film (Fig. $2.25 \mathrm{~b}-2$ ).

For case (1), the trivial solution for $r_{s c}$ is $r_{s c}=r_{d}=$ constant.

For case 2, assuming that the droplet maintains the shape of a spherical cap of constant apparent contact angle on the remnant film as it retracts, and the liquid volume in the film is neglected and taking $\theta=\theta_{r}$ and $\mathrm{rd}=$ constant, the following equation can be rearranged as [53]:

$$
r_{s c}(t)=r_{d}\left(1-{\sqrt{\frac{t}{\tau_{C D A}}}}^{1 / 3}\right.
$$

Where the time to fully deplete the droplet $\tau_{C D A}$ is given by [85]

$$
\tau_{C D A}=\left(\frac{V_{0}}{r_{d}^{2} K}\right)^{2}
$$

The predicted depletion time is independent of retraction angle, $\theta_{r}$, which is expected from the fact that the drawing area is constant.

\section{Decreasing Drawing Area (DDA), Fixed Contact Angle}

If the actual liquid contact line retracts, or if the remnant film is too thin to accommodate appreciable liquid flow, then the effective drawing area decreases as the droplet is depleted. In this case, the general model of Eq. (1-39) becomes nonlinear, inhomogeneous, and Volterra- type integral equation. If the apparent contact angle of the droplet remains constant as the contact line retracts, this equation admits a surprisingly simple equation with striking similarity to the CDA solution [53]. 


$$
r_{d}(t)=r_{0}\left(1-\sqrt{\frac{t}{\tau_{D D A}}}\right)
$$

Where $r_{0}$ is the initial contact radius.

$$
\tau_{D D A}=9 \tau_{C D A}
$$

A characteristic prediction of the time-dependent contact radius for this limiting case is also given in Fig. 2.26.

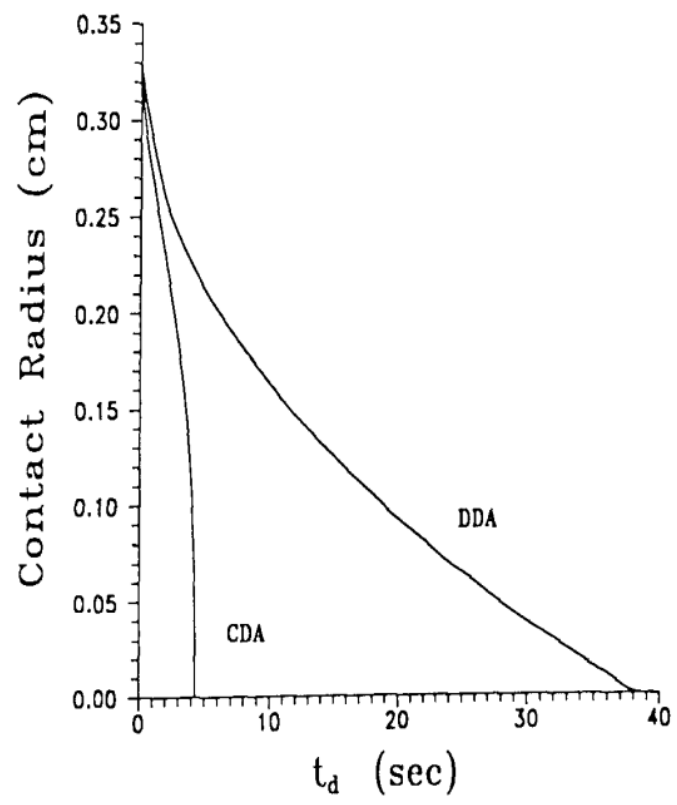

Fig. 2.26 Characteristic prediction for constant and decreasing contact area limiting cases [53]

From this work it is expected that the behavior of the apparent contact radius is dictated primarily by the droplet depletion process rather than by the droplet spreading process. The time dependence of the apparent contact radius will depend upon the drawing character (CDA vs DDA which in turn depend on liquid and powder characteristics) and on the behavior of the apparent contact angle. 
Moreover, in [80] instead of an assemblage of parallel cylindrical capillaries, which are commonly employed, a swarm of spherical particles have been considered for the modeling purpose of such media.

In this study, a general treatment of the rate of the penetration of a fluid into porous media is derived from Darcy's law which is independent of any particular model as follows [80].

$$
V=-\frac{k}{\mu} \frac{d p}{d z}
$$

Where $\mathrm{k}$ is the permeability and $\mathrm{p}$ the pressure.

Expanding the Darcy's law to regard a cross sectional area A of the porous medium through which incompressible fluid of density $\rho$ and viscosity $\mu$ is advancing in a direction normal to the area $\mathrm{A}$ with the instantaneous velocity $\mathrm{V}$ at a penetration distance $\mathrm{z}$ of the fluid front and S the specific area of the solid/ fluid interface, i.e. the internal surface area per unit volume of porous material gives rise to the following equation.

$$
V=\frac{d z}{d t}=\frac{2}{3} \frac{y a}{3 \mu \Omega}
$$

Where

$$
\Omega=\Omega_{H}(y)=\frac{2\left(3+3 y^{5}\right)}{3\left(2-3 y+3 y^{5}-2 y^{6}\right)}
$$

The Eq. (1-40) has a distinct advantage over other models that take a porous material as an assemblage of capillaries with variety of radii in that the velocity of the penetration $\mathrm{V}$ in Eq. (1-40) is expressed in terms of particle radius. In contrast, when 
applying Washburn's equations for example, the relation of the cylinder radius to the particle size remains unknown.

The wetting theory presented in [80] has been highly idealized. In practice there may be several complicating effects. Volume changes occurring within the porous medium during imbibition, entrapment of air as liquid rises into porous medium, the presence of electric double layers at the particle surface are few examples which might contribute to inaccuracy of the developed model.

Abraham Marmur in [56] has compared the penetration of a liquid from an infinite reservoir into a porous medium and into an equivalent system of capillaries with respect to liquid-vapor and liquid-solid interfaces. While a liquid-vapor interface is continually created by reexposing the liquid to vapor inside the porous medium and at its boundaries (termed reexposure effect), this feature of penetration is absent in the case of penetration into capillaries, where the liquid-vapor interfacial area may fluctuate but not continually increase. The model system considered here is shown in Fig. 2.27.

In order to identify the equilibrium situations, to which the system is driven by interfacial interactions, an expression for the free energy of the system is formulated and then minimized.

To develop the expression for the free energy, the various interfaces between different phases are considered with the aid of Fig. 2.27. Four different liquid-vapor interfaces and two solid-liquid interfaces regarded in this model include the interface between the liquid and the vapor outside of the porous medium (LV-1 in Fig. 2.27 c), the 
liquid-vapor interface at the "upper" side of the porous medium (LV-2 in Fig. $2.27 \mathrm{c}$ and e), the liquid-vapor interface at the "lower" side of the porous medium (LV 3 in Fig. 2.27 e), the liquid-vapor interface inside the porous medium, at the periphery of the liquid cylinder (LV-4 in Fig. 2.27 e), the solid-liquid interface inside the porous medium (SL-1 in Fig. 2.27 c), and the solid-liquid interface at the base of the drop (SL-2 in Fig. 2.27 c) $[56]$.

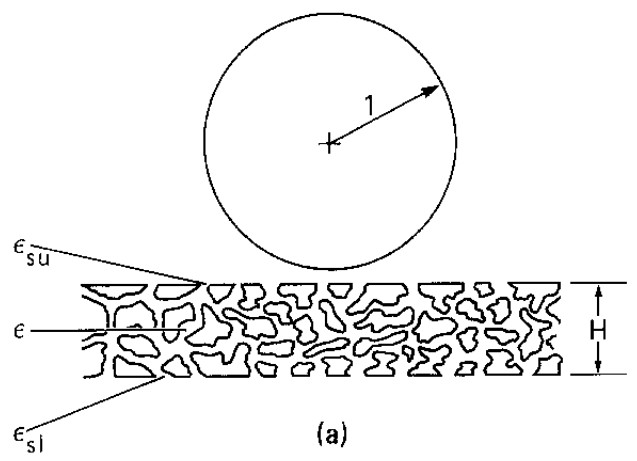

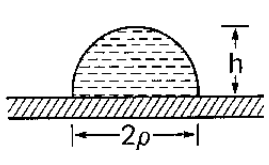

(b)

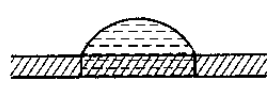

(d)

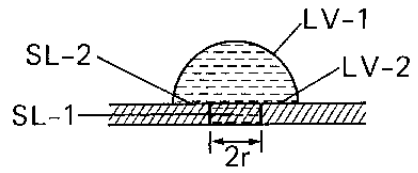

(c)

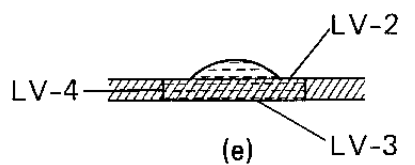

(f)

Fig. 2.27 (a) The drop and the thin porous medium prior to contact (b) initial spreading over the surface of the porous medium, which may coincide with (c) initial penetration into the porous medium (d) basal penetration (e) a "halo" (f) complete penetration [56]

The main idealization of the present thermodynamic model lies in the assumption of uniform penetration throughout the thickness of the porous medium. This assumption makes the calculations easier; however, it is not indispensable. The mechanism responsible 
for most of the predictions of the model is the reexposure effect, which stems from the thinness of the porous medium and is independent of the assumption of uniform penetration. Thus, the qualitative conclusions should also hold for some degree of nonuniformity in penetration, although the numerical results may change.

In this study, he found out that the reexposure effect (i.e. the continual increase in the liquid-vapor interfacial area inside the porous medium and at its surfaces as penetration proceeds) may be sufficiently dominant for thin porous media to prevent complete penetration even for $0=0 \%$ and drops of different sizes of the same liquid may behave very differently when penetrating into the same porous medium.

Interconnectivity of pores in a powder bed

Porous materials often possess at least some degree of pore interconnectivity. Such interconnectivity is expected to increase the rate of depletion since the effective number of drawing pores generally increases as liquid is imbibed into the porous material.

In the model developed by Denesuk et al. [53] where porous material is considered as a bundle of capillaries with various diameters, the total number of drawing pores per unit drawing area at the surface of the porous material is defined as $\rho_{p}$. In this work, the interconnectivity of pores is taken into account by increasing the pore density per unit area.

Considering the picture below, in the ideal case the total area density of effective drawing pores is assumed to oscillate between $\rho_{p}$ and $\rho_{5 p}$ in that the contribution of branches (c) and (d) to liquid depletion will vanish as the liquid in these regions meet the corresponding liquid fronts from the neighboring elements. In this work, these arguments 
suggest the incorporation of pore interconnectivity into the depletion model by a substitution of $3 \rho_{p}$ for $\rho_{p}$. According the aforementioned equations, this replacement implies a factor of 9 decrease in the corresponding depletion time.

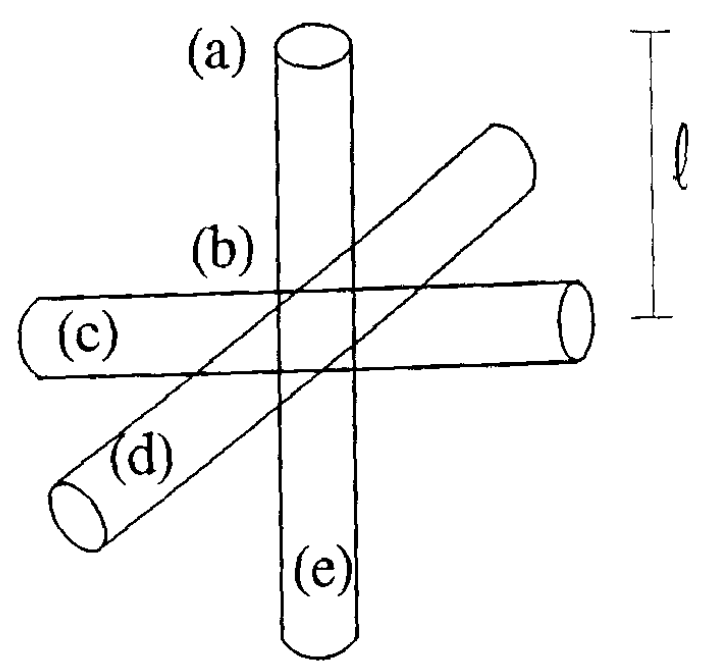

Fig. 2.28 Schematic representative element of an interconnected pore geometry [53]

In [86], Clarke et al has developed a model for the spreading and penetration of liquid droplets on porous surfaces. In this study, the droplet spreading rate is represented by the molecular-kinetic theory according to the equation below.

$$
\frac{\partial r}{\partial t}=\frac{2 K_{S}^{0} h \lambda}{\mu v} \sinh \left(\frac{\gamma\left(\cos \left(\theta_{0}\right)-\cos (\theta)\right)}{2 n k_{B} T}\right)
$$

Where $r$ is the instantaneous spreading droplet radius (shown in Fig. 2.29), $K_{S}^{0}$ is a molecular jump frequency at $\mathrm{n}$ sites of solid/liquid interaction per unit area of the substrate, $\mathrm{h}$ is Planck's constant, $\mathrm{v}$ is the molecular volume, $\lambda\left(=\frac{1}{\sqrt{n}}\right)$ is the molecular jump length, $\mu$ is the viscosity, $\lambda$ is the liquid surface tension, $\theta_{0}$ is the equilibrium contact angle, $\theta$ is the instantaneous dynamic contact angle, $\mathrm{kB}$ is Boltzmann's constant, and $\mathrm{T}$ is the 
temperature. Axes definition for the spread and absorption of a sessile droplet is illustrated in Fig. 2.29

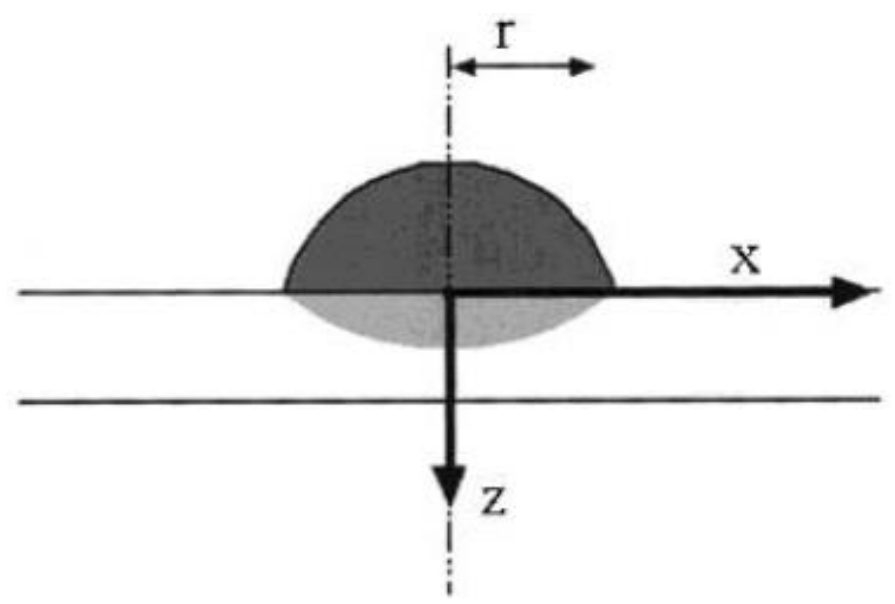

Fig. 2.29 Axes definition for the spread and absorption of a sessile droplet at time $t$ [86]

Eq. (1-48) is shown to model the droplet spreading rate over a non-porous substrate [87]. To model the droplet spreading on a porous substrate, the condition of constant droplet volume is relaxed using the following equation which relates the droplet radius to the contact angle and the volume.

$$
r=\left[\frac{3 V}{\pi} \frac{\sin ^{3}(\theta)}{2-3 \cos (\theta)+\cos ^{3}(\theta)}\right]^{1 / 3}
$$

In their modeling, Darcy's law is used to describe the droplet penetration into the porous medium as follows.

$$
\nabla p=\frac{\mu}{k} u
$$

Where $\nabla p$ is the pressure gradient, $\mathrm{k}$ is the permeability, $\mathrm{u}$ is the mean liquid velocity in the pore. In this modeling, the parameters which need to be experimentally 
determined include $K_{S}^{0}, \mathrm{~h}, \mathrm{v}, \lambda\left(=\frac{1}{\sqrt{n}}\right)$. It must be noticed that there is no definitive way of predicting the values of these parameters for a given solid/liquid system, and so predicting wetting behavior from independently measured quantities [88]. In the modeling developed by Clarke et al the initial droplet velocity and its influence on droplet spreading/penetration dynamics are overlooked.

Starov and co-workers proposed an approach that combines the lubrication theory and Brinkman's equation to model the droplet spreading and penetration [89-91]. In their modeling, the spreading and penetration were assumed to occur on different time scales [92] as shown in Fig. 2.30.

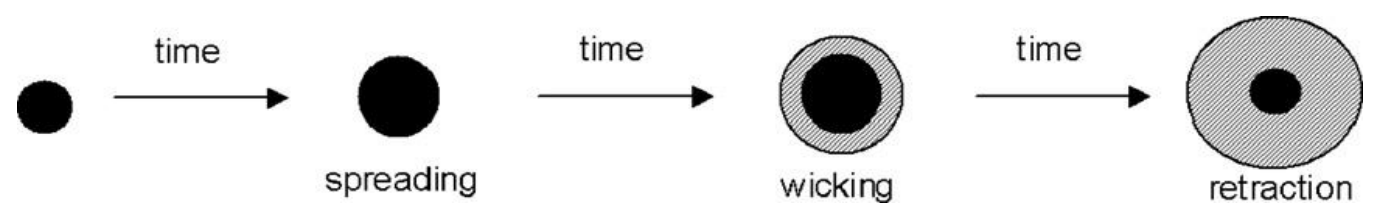

Fig. 2.30 Spreading and penetration behavior studied by Starov et al [89, 90, 93]

Yip et al [94] developed a one-dimensional model to describe droplet penetration process in process media. With assumptions of the constant contact radius ( $\mathrm{R}$ as shown in the figure below) and the fluid incompressibility, they have employed Darcy's law to derive the following equation for the volume of droplet remaining on the surface at any given time $t$.

$$
V(t)=V_{0}-2 \pi R^{2} \emptyset\left(\frac{k p_{c}}{2 \mu \emptyset} t\right)^{0.5}
$$


Where $\mathrm{V}_{0}$ is the droplet initial volume, $\mathrm{R}$ radius of the wetted area, $\varnothing$ porosity of the porous medium, $\mathrm{k}$ is the permeability, $\mathrm{p}_{\mathrm{c}}$ is the capillary pressure, and $\mu$ is the fluid viscosity as schematically shown in Fig. 2.31 .

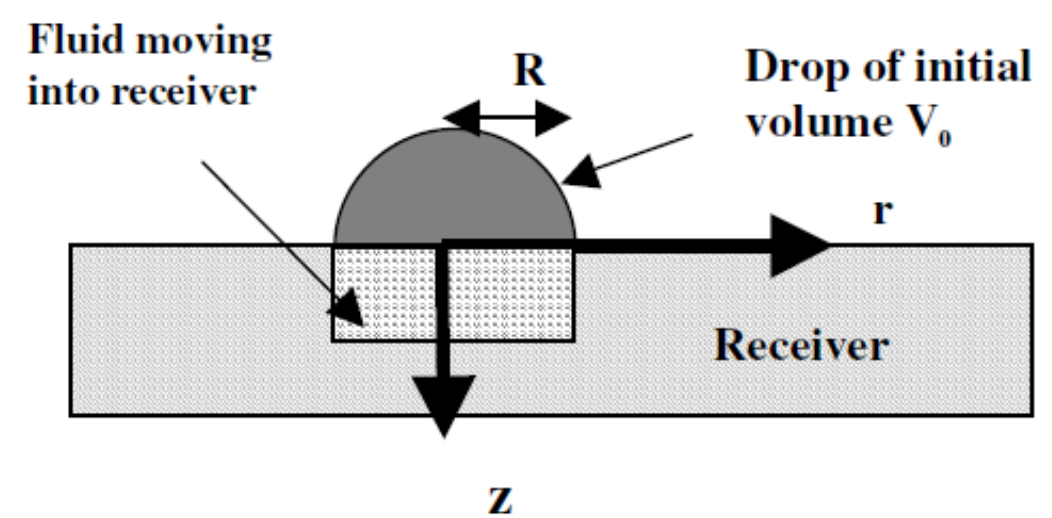

Fig. 2.31 Geometry of droplet penetration by a porous medium [94]

2.4.3 Binder droplet-powder bed interaction modeling (dynamic phase)

In the context of BJ-AM process, there exist very limited researches about the binder droplet-powder bed interaction modeling. Holman et all studied the simultaneous spreading and penetration of an aqueous polymer solution droplets deposited on porous ceramic beds [95]. In this work, a model based on basic spreading kinetics and Denesuk's equation [53] for droplet penetration rate was developed in the attempt to predict the maximum droplet extension.

In the modeling developed by Holman et al, the instantaneous radius of a spreading droplet and the droplet penetration rate were characterized using the equations (1-51) and (1-52), respectively. 


$$
\begin{gathered}
\mathrm{r}(\mathrm{t})=\mathrm{a} \cdot(\mathrm{b}+\mathrm{t})^{\mathrm{n}} \\
V_{p}(t)=\frac{K}{2} \int_{0}^{t} \frac{r^{2}(t)}{\sqrt{t}} d t
\end{gathered}
$$

Where

$$
K=\pi \cdot a_{p} \sqrt{\frac{\gamma \cdot \cos \theta \cdot R_{p o r e}}{2 \eta}}
$$

$\mathrm{r}(\mathrm{t})$ is the instantaneous radius of the spreading droplet at time $\mathrm{t}, \mathrm{a}, \mathrm{b}$, and $\mathrm{n}$ are constants, $\mathrm{R}_{\text {pore }}$ is the pore radius (mean pore radius from the BET measurements), $\mathrm{a}_{\mathrm{p}}$ is the volume fraction of the pores, $\gamma$ is the surface tension of the liquid, $\theta$ is contact angle of the binder droplet on the material, and $\eta$ is the droplet liquid viscosity. In this approach, it is assumed that the contact angle between the droplet and the powder material will have the equilibrium contact angle at which spreading stops. In other words, the contact angle starts with an advancing value, and after reaching the equilibrium value progresses to the receding contact angle. For this one instant, the liquid droplet will assume the equilibrium shape for the volume of the droplet remaining on the surface. The radius of the remaining droplet volume on the surface is calculated from Eq. (1-49). Therefore, the maximum droplet spreading radius (or diameter) is found by determining the point at which the equilibrium radius and the instantaneous radius of the spreading droplet are equal [95]. Similar to Clarke's work [86], the droplet initial velocity is not considered in the modeling developed by Holman and co-workers. 
In his Ph.D. dissertation [96], Tailin Fan developed one-dimensional model for the impact penetration of a droplet into a powder bed of a certain type. Under the circumstances investigated in his study, it is assumed that the impact of a liquid droplet on a loose powder bed creates a ball-shaped particle aggregate sitting on the bottom of a bowl-shaped crater. In other words, it is assumed that after impact the particles start to enter the droplet and generate a moving mixture of powder particles and the droplet in the powder bed as shown in Fig. 2.32. The developed model is to predict the penetration depth of the ball-shaped particle aggregate.

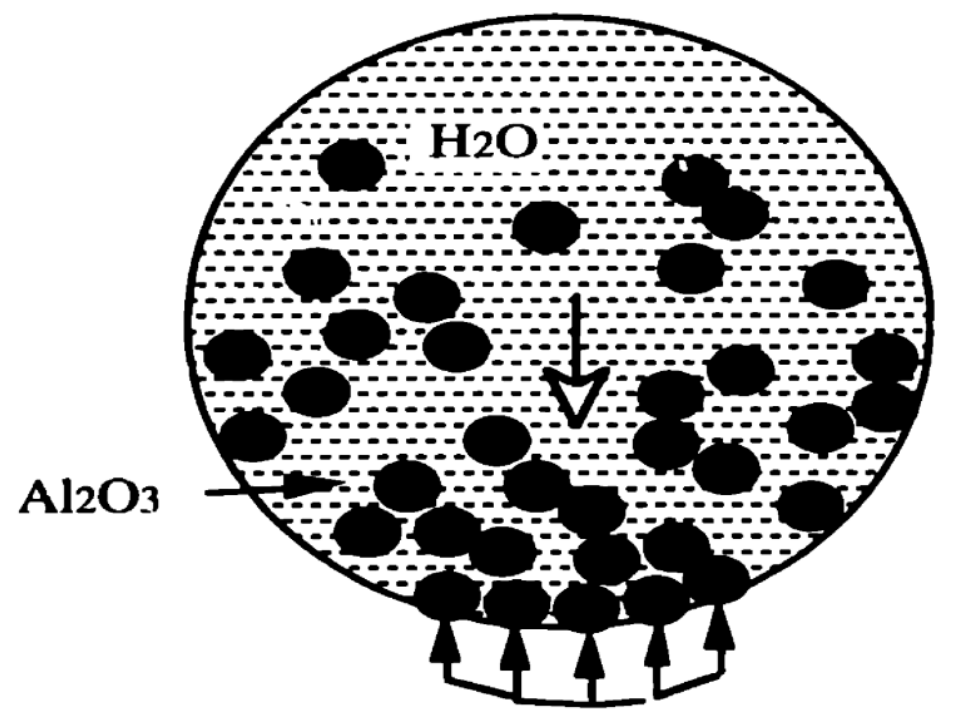

Fig. 2.32 Illustrating Al2O3 particles entering a water droplet 


\section{CHAPTER 3}

\section{RESEARCH OBJECTIVES AND CORRESPONDING PROCEDURES}

\section{1 Introduction}

Currently, fabricating parts using BJ-AM process relies greatly on trial-and-error approach due to lack of fundamental understanding of this process which in turn leads to inefficient process development and poor part quality control. In order to take the best advantage of this process and turn it into an outstanding option which can be used in different industries, extensive and systematic investigation of this technology in different aspects from the binder behavior within powder bed to post processing is required. The present research project aims to improve the fundamental understanding of the feature formation in BJ-AM technology in the most basic levels and establish predictive models describing different aspects of green part fabrication process.

3. 2 Feature formation in Binder Jetting Process in the most fundamental level

From the extensive overview of the literature, it was found out that very limited attempts have been made to fundamentally investigate the feature formation phenomenon in this technology. The feature formation in Binder jetting additive manufacturing starts with delivering individual binder droplets in the designated areas of the powder bed. Depending on the droplet spacing in the $\mathrm{x}$ and $\mathrm{y}$ directions (Fig. 3.1), deposition rate, and 
also rate of binder diffusion in the powder bed, the deposited droplets might coalesce before the droplet penetration completes.

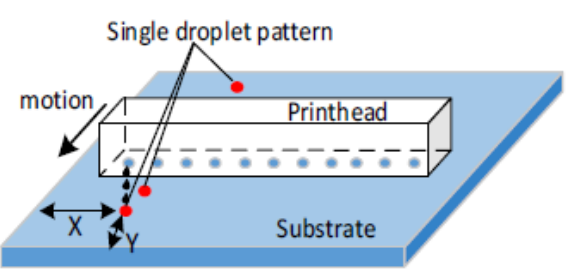

a. Schematics of droplet spacing

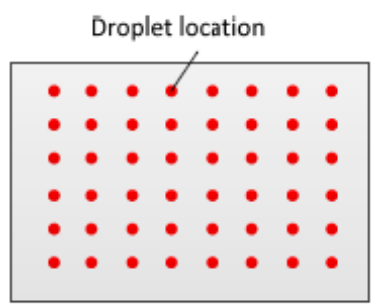

b. Droplet pattern across the substrate

Fig. 3.1 Droplet deposition pattern in Binder Jetting process

Thereafter, the droplets (Coalesced or singles drops) migrate into the powder bed due to the driving forces such as capillary pressures, gravity, etc. until the equilibrium condition in which binder migration in the powder bed stops is reached. Fig. 3.2 schematically displays the equilibrium condition between a binder droplet and powder bed in a larger scale. An accurate physics-based model to predict the binder behavior inside the powder bed for this stage of feature formation in Binder Jetting process is of significant importance in that it will determine the equilibrium saturation which corresponds to the optimal saturation level. Once the equilibrium saturation is adopted for printing components in practice, it will result in the best fabrication qualities. 


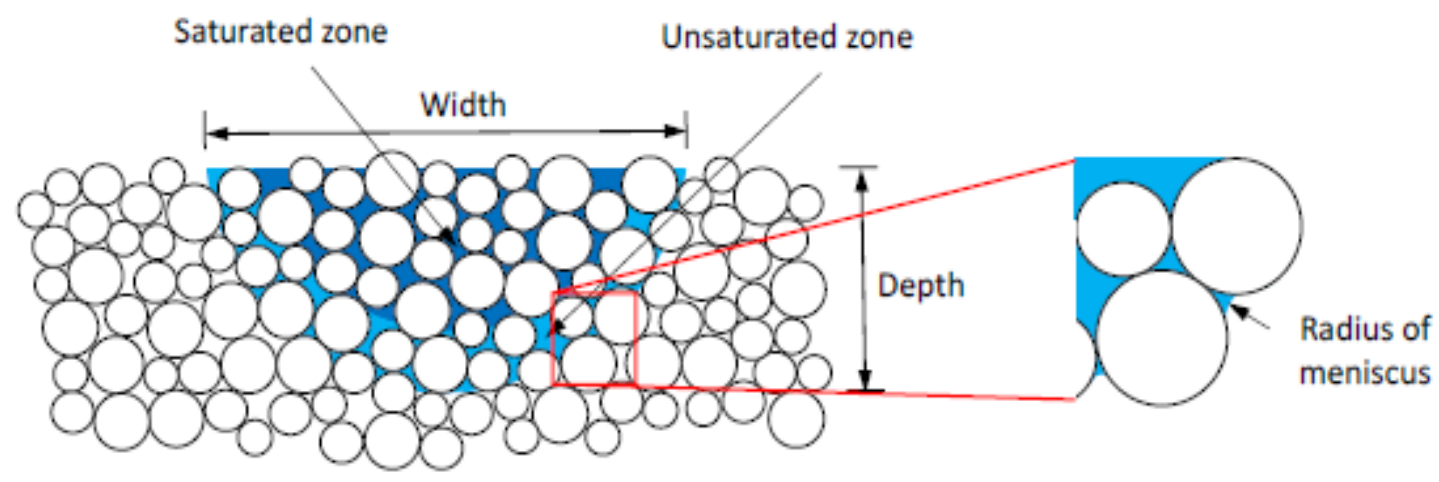

Fig. 3.2 Equilibrium condition between binder and powder bed

Furthermore, the profile of area saturated by a single droplet after reaching to equilibrium condition can be realized from the established physics-based model and the droplet spreading/penetration kinetics. Such profile (i.e. permeation profile) characteristics (W and D dimensions in Fig. 3.3) have multiple implications to Binder Jetting process. Firstly, the permeation profile largely determines the minimum feature resolution of the fabrication process and consequently influence the geometrical accuracy. Secondly, the liquid distribution within the powder bed influences the strength of the green parts in combination with the other process control parameters. Lastly, such information is also critical in determining the effect of binder on the microstructural and mechanical qualities of the densified final parts. Therefore, the quantification of the binder permeation is of critical importance to the understanding of Binder Jetting process. 


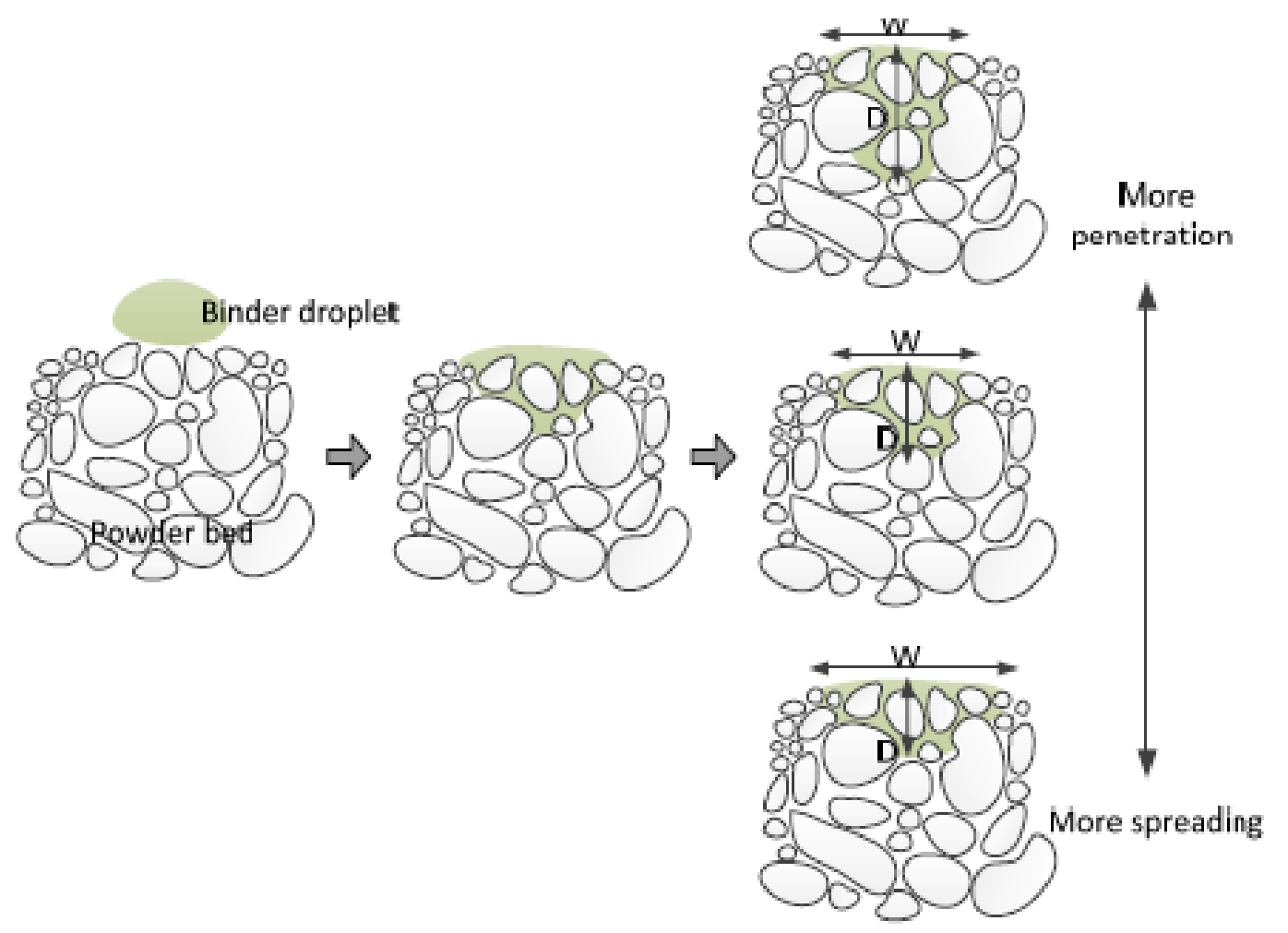

Fig. 3.3 Binder droplet permeation in powder bed

Therefore, based on the descriptions the following research objectives and goals will be specifically investigated in the present dissertation:

3. 3 Developing a physics-based model for the binder/powder bed interaction in the equilibrium phase

In this section, liquid binder behavior within a powder bed including the driving forces for binder migration and the mechanism(s) that causes the binder penetration to stop as well as different parameters influencing binder distribution inside the powder bed will be thoroughly investigated. In this regard, the extensive literature available particularly in the areas such as civil engineering, wet granulation, and 2D-ink printing, will be quite advantageous in that in all of these processes permeation of a liquid into a porous medium is studied. While the basic principles about interaction between a fluid and a porous 
medium (e.g. driving forces for fluid migration, terminating mechanisms for liquid permeation, etc.) in such processes will be utilized, due to the unique process characteristics such as the layer-wise process, the selective application of binder and the in-process binder drying, the entire established knowledge with these processes will not be applicable in all aspects of Binder Jetting technology. Therefore, a new physics-based model will be developed to accurately predict the interaction between liquid binder and powder bed in BJ process in the equilibrium state. This model will be quite helpful in estimating the equilibrium condition in which the liquid binder will no longer migrate and also establishing a droplet permeation profile inside the powder bed. Furthermore, the accuracy of the developed model will be validated by empirically measuring the equilibrium saturation. For this purpose, at least two types of power materials will be considered for conducting the experiments. ExOne M-Lab machine will be utilized to spread the powders and a $0.5-20 \mu \mathrm{L}$ micropipette will be employed to deposit individual droplets. The dimensions and weights of such samples will be measured using an optical microscopy and a $(0.001 \mathrm{~g})$ digital scale respectively. Additionally, single tracks, multiple tracks, and cubic parts will be printed out using M-Lab printer so as to examine the equilibrium saturation in practice during feature formation in binder jetting process. The schematic of single-track and multiple-tracks experiments has been shown in Fig. 3.4. 


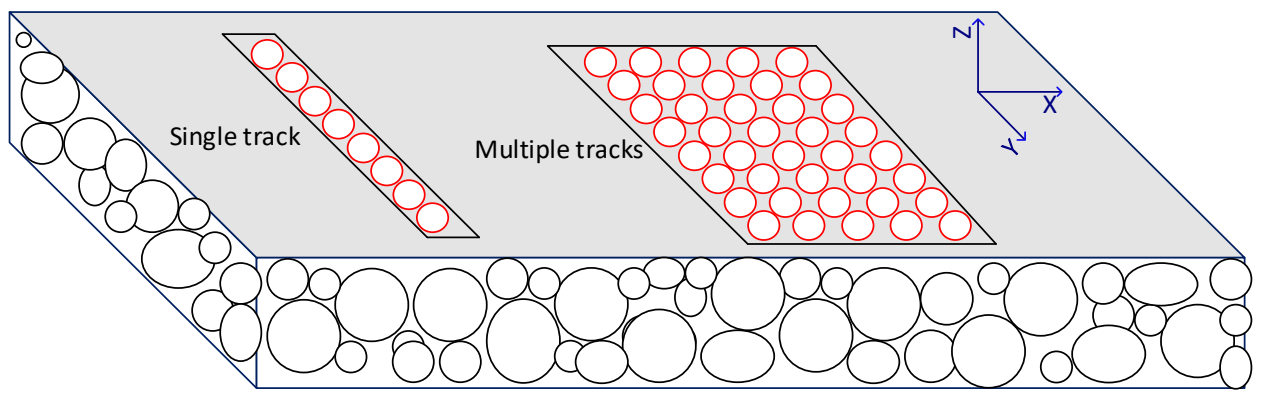

Fig. 3.4 Schematic of single track and multiple tracks experiments

Single-track experiments in which only one nozzle of the printhead is employed for printing will provide more accurate information about the equilibrium saturation whereas equilibrium saturation obtained from multiple-track printing will include droplet poisoning errors of the printer in the $\mathrm{Y}$ direction and the effect of deposition delay between consecutive tracks as well. Moreover, the measurements from cubic parts will provide useful knowledge about the overall equilibrium saturation and process of feature formation in Binder Jetting Technology using M-Lab ExOne machine. This overall equilibrium saturation will take into consideration any trapped powder between the droplets in consecutive layers as shown in Fig. 3.5.

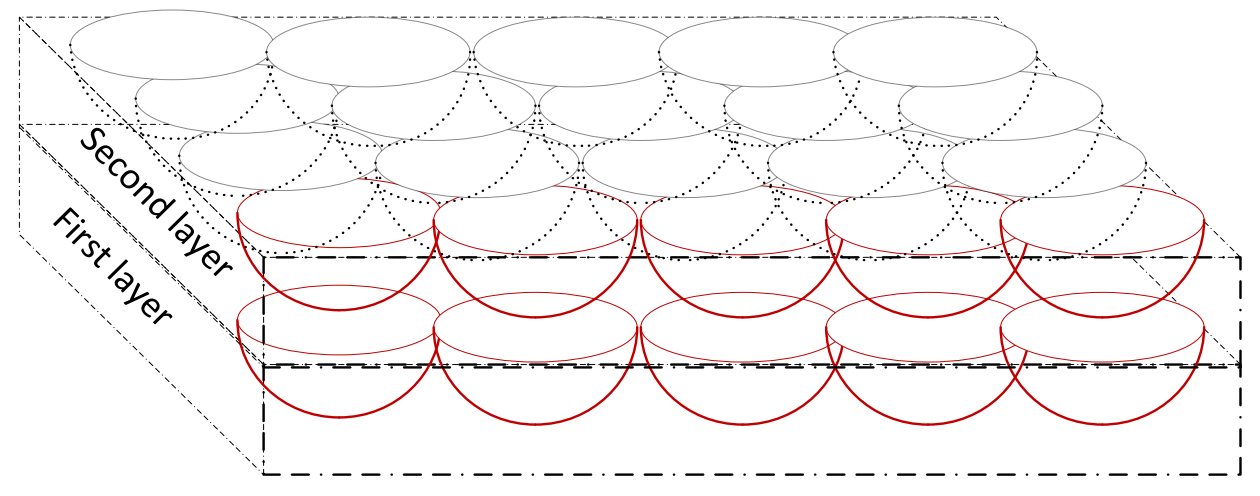

Fig. 3.5 Schematic of feature formation in Binder Jetting Process 
3. 4 Investigating the effect of different parameters on binder-powder bed interaction in the dynamic phase

After a droplet impact on the powder bed surface, different parameters might affect the droplet-powder bed interaction in the dynamic phase before the droplet completely penetrates the powder bed. In order to model binder-powder bed interaction in the dynamic phase, these parameters and their effect on the interaction need to be analyzed in the context of BJ-AM technology. Therefore, different parameters including droplet impact velocity, powder particle size, and particle size distribution will be experimentally explored for their effect on the binder droplet-powder bed interaction and as a result, on the part quality. To investigate the droplet impact velocity, samples with various printing speeds will be fabricated and their dimensional accuracy will be evaluated. Quantitative correlations will be established between the part dimensional accuracy and the printing speed. Moreover, the effect of the powder particle size characteristics on the droplet/powder bed interaction will be analyzed by real-time imaging of the interaction, from the moment of impact to equilibrium status.

3.5 Developing a theoretical model to describe the droplet-powder bed interaction in the dynamic phase

With the insight from the systematic experiments on the effective parameters, a theoretical model for describing the droplet-powder bed interaction in the dynamic phase will be established based on droplet spreading/penetration kinetics, powder bed characteristics and binder physical properties. 
Experimental work will be conducted in order to evaluate the accuracy of the theoretical model with variety of powder materials. A micropipette will be used to deposit individual droplets and the real-time dynamics of the droplet-powder bed interaction will be captured. For this purpose, a real-time imaging system using Fastec Imaging HiSpec 2 high speed camera will be developed. As shown in Fig. 3.6, the system consists of a micropipette (2-10 microliter droplet volume size), the high-speed camera, mechanical fixtures for holding the micropipette and the camera, and a light source (150W Fiber Optic Dual Gooseneck Microscope Illuminator). Individual droplets of $2 \mu \mathrm{l}$ volume will be deposited using the setup, and real-time flow dynamics of the impacting droplets on different powder beds will be recorded. The final diameter and depth of the resulting liquidpermeated features will be measured by an optical microscope (Olympus MX51 with Olympus QColor 5 digital camera) and a digital caliper. The ExOne M-Lab printer will be used for forming the powder beds with the roller spreading speed of $2 \mathrm{~mm} / \mathrm{s}$. Using the developed model, the profile of the powder bed saturated area by a single droplet will be estimated for any given material system. After validation with experiments, the theoretical model can be incorporated into the printing process so as to improve the quality of the printed parts by enhancing the process controllability. 


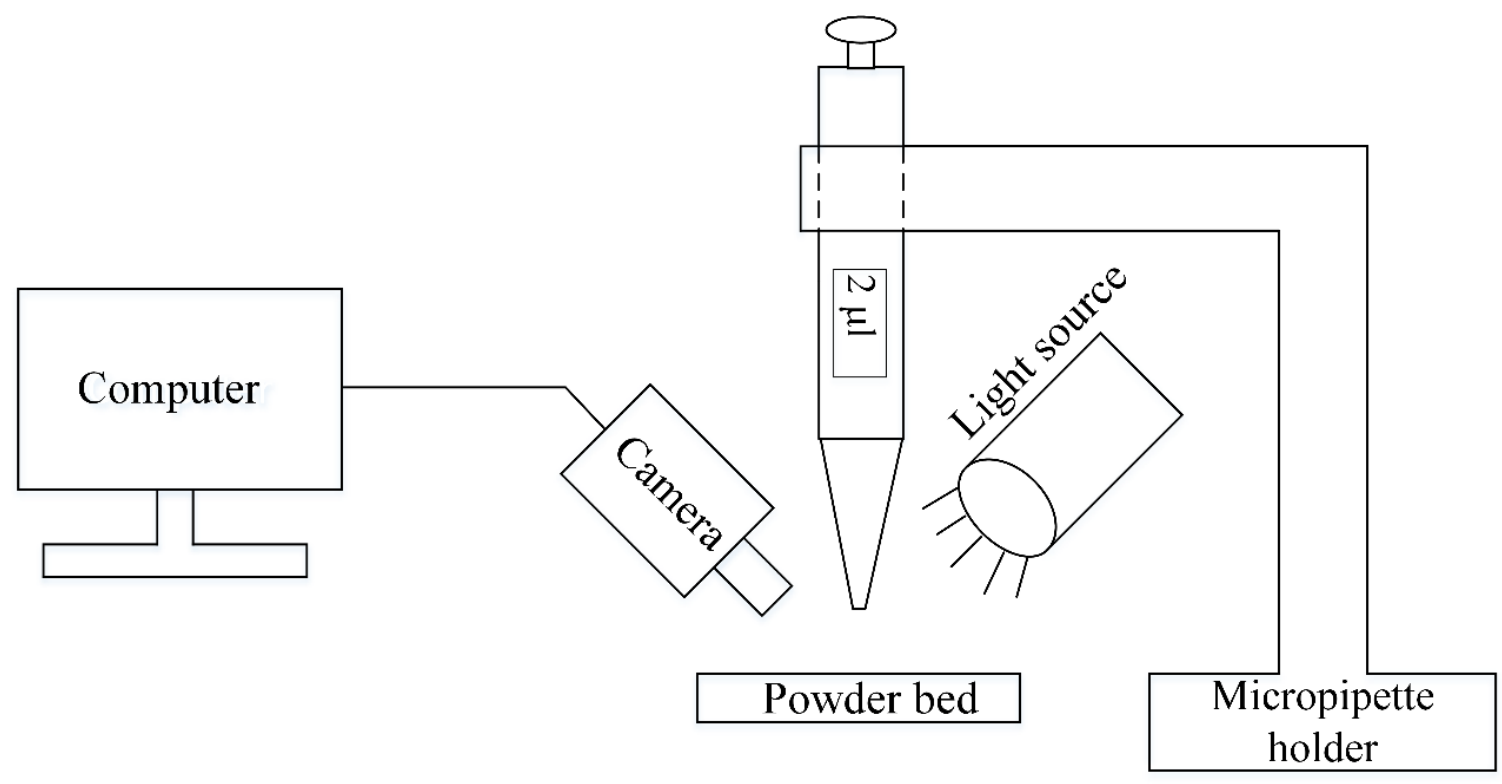

Fig. 3.6 The schematic of high-speed imaging setup 


\title{
CHAPTER 4
}

\author{
A NEW PHYSICS-BASED MODEL FOR EQUILIBRIUM SATURATION \\ DETERMINATION IN BINDER JETTING ADDITIVE MANUFACTURING \\ PROCESS
}

\subsection{Introduction}

The binder jetting additive manufacturing (BJ-AM) process is one of the additive manufacturing (AM) processes that has been broadly adopted for various applications. Due to the use of the binders for the geometry creation at room temperatures, the BJ-AM possesses various advantages over other AM processes, such as the elimination of thermally induced defects (e.g. distortion, unwanted grain growth, etc.) and wide compatibility with various materials. Some of the exotic materials such as Inconel 718 [97], copper [98], zirconia [99], silicon carbide [12], barium titanite [58], calcium phosphate [8, 32] and hydroxyapatite [10] have been used by the BJ-AM processes, which can be challenging even for some of the other AM processes. In BJ-AM process, droplets of the liquid binder are delivered to the designated surface area of the spread powder bed through a print-head. After the deposition of the binder, the entire surface of the powder bed is exposed to certain amounts of thermal energy commonly by means of heating lamps in order to introduce adequate mechanical strength into the printed structures to withstand the shearing and gravitational forces involved in the consequent printing processes. These 
steps are repeated for each layer until the entire samples are completed. The geometrical qualities and the structural integrity of the green parts strongly rely upon both the quantity of the deposited binders and the characteristics of the binder-powder bed interactions [100].

In the BJ-AM process, once the liquid droplets are deposited on the desired locations, the droplets will start to migrate into the powder pores under the influence of both capillary attraction and the surface tension-induced pressure gradient across the binder droplet meniscus formed between the binder and air as schematically shown in Fig. 4.1 $[13,14,52,57,101,102]$. The surface tension-induced pressure gradient decreases as the binder penetrates further into powder bed, and eventually disappears when there is no binder left on the powder bed surface. Therefore, during the binder permeation process the primary driving mechanism for the binder movement inside the powder bed is the capillary pressure. The state when the binder stops migration within the powder bed due to the balance of the capillary pressure $\left(\mathrm{P}_{\mathrm{c}}\right)$ across all binder-air interfaces (meniscus) establishes the equilibrium state of the process (Fig. 4.2) [57, 101].

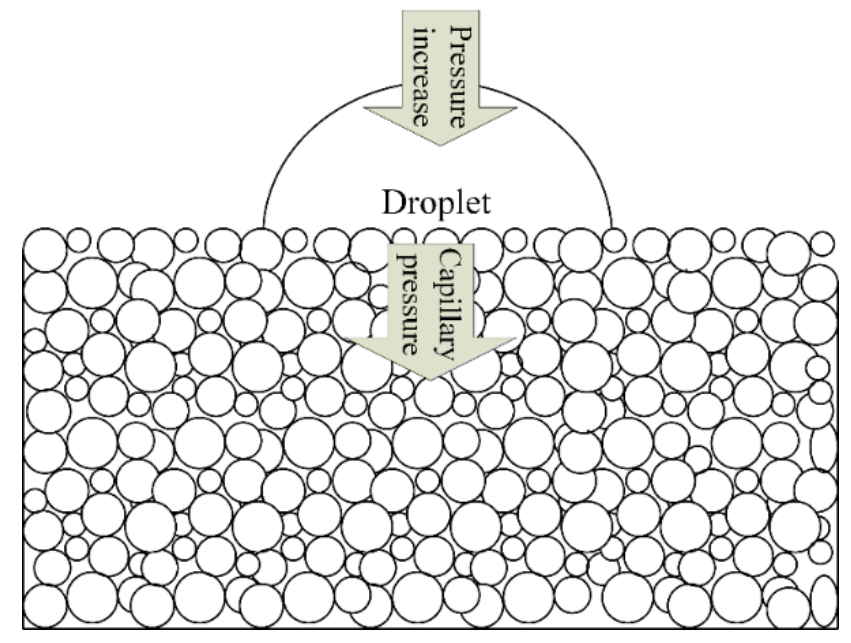

Fig. 4.1 Schematic presentation of a binder droplet on the powder surface 


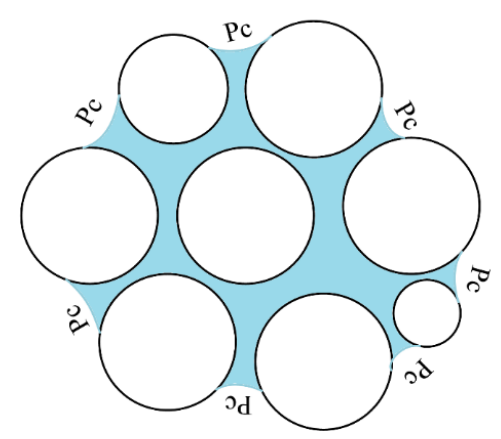

Fig. 4.2 Equilibrium state in the pore scale (Pc: capillary pressure)

Under the equilibrium conditions, the binder saturation level, which is defined as the ratio of binder volume to the pore volume in a pre-defined envelope of powder bed, is of significant importance in the BJ-AM process. This equilibrium saturation determines the optimal saturation level required for the successful creation of green parts [103, 104]. If the saturation imposed by the printer control settings is much greater than the equilibrium saturation, the excess liquid binder tends to migrate out of the designated boundaries of the feature to be printed. On the other hand, in the opposite case, the printed part won't have sufficient mechanical strength due to the lack of sufficient binder phase, which results in weak bonding between the particles as well as between successive layers. Several works have experimentally demonstrated that the binder level plays a key role in determining the part dimensional accuracy and mechanical performance in BJ-AM process $[14,26,58$, 105]. Therefore, precise estimation about the equilibrium saturation for a given powder bed and liquid binder is highly desired for the design of the BJ-AM process and the optimization of the qualities of the printed parts. Currently, the selection of the binder saturation levels in the BJ-AM process is largely based on trial and error approach, and very limited attempts have been reported for the prediction of the equilibrium saturation levels. In [104], the equilibrium saturation estimations from the model that was developed based on previously proposed imbibition-drainage characterization does not appear to be 
in good agreement with the experimental results, which was suggested to be attributed to several potential factors such as the hysteresis between the drainage and imbibition characteristics [103]. In order to understand the interactions between liquid binder and the porous powder bed effectively, a fundamental model that describes the behaviors of a fluid in a porous medium for the BJ-AM process is needed.

There exist relevant application areas where displacement of a fluid by another fluid in the presence of porous structures is considered, such as oil recovery [106], water infiltration into the soil [107] and 2-D inkjet printing [86]. Within these areas, an abundance of literature covers various subjects including displacement patterns and computer simulations [108], displacement mechanisms in the pore (micro) scale and macro scale [109], spreading (lateral displacement) and penetration (vertical diffusion) rates [53, 102], wettability and its effect on fluid migration [110], simulation of fluid migration inside porous medium [111], and saturation dependence on the capillary pressure [104]. While many of these works provide useful references to the understanding of the BJ-AM process, the equilibrium saturation levels of a finite-volume fluid body in the presence of another fluid inside a porous medium has not been dealt with in much details in the context of the BJ-AM process.

In the present work, a physics-based model is established to estimate the average capillary pressure in the equilibrium state. The model can be employed to predict the average saturation levels with the calibration of the experimental saturation-capillary pressure characterization curve. The accuracy of the model was evaluated through experimentation, and the results were discussed in detail. 


\subsection{Modeling of binder saturation}

During the BJ-AM process, the binder droplets are delivered from individual nozzles in the printhead and subsequently deposited on the powder bed surfaces. Upon impact on to the powder bed surfaces, the droplets experience some turbulences due to inertial forces [86]. Depending on the velocity, density, surface tension, and volume of the droplet, splashing might occur upon the impact. In [112], it was demonstrated that the splashing phenomenon is more likely to occur when the Weber (We) number is $>50$. For BJ-AM process, The We number can be calculated from:

$$
\mathrm{We}=\rho r v^{2} / \gamma
$$

where $\rho$ is the density of the binder material, $\mathrm{r}$ and $v$ are the radius and velocity of the droplet, respectively, and $\gamma$ the surface tension of the liquid binder. From the printer specifications and binder physical characteristics used in the present study, the We number is calculated to be less than the threshold amount $(\mathrm{We} \approx 50)$ for splashing. Therefore, although initial disturbance and wave-like curvature might be present on the droplet surface after impact $[86,113]$, no splashing is expected to occur. Furthermore, the momentum will be dissipated very quickly $\left(10^{-4} \mathrm{~s}\right)$, and capillary force is expected to be the only dominant driving force thereafter (after $\left.10^{-3} \mathrm{~s}\right)[53,104]$.

In order to establish the equilibrium model that properly represents the physical reality of the binder-powder bed interaction, the binder penetration pattern must be evaluated first, as it could significantly influence the uniformity of the saturation levels within the permeated regions. From the literature, fluid-fluid displacement in the presence of a solid surface can be categorized into two types as either drainage or imbibition based 
upon the system's wettability $[110,114]$. While drainage is described as the fluid migration mechanism in which the penetrating fluid is less wetting to the solid surface than the displaced fluid, imbibition refers to the opposite case in which the penetrating fluid is more wetting. It is obvious that imbibition is more representative for the description of the binder permeation process in BJ-AM, since the displacing fluid (liquid binder) is more wetting than the fluid being displaced (air). The most critical factors that determine the displacement patterns are the viscosity ratio $M$ and the capillary number $C a$, which are two dimensionless factors that indicate the relative viscosity and relative viscous force respectively [114-116]. The definitions for $M$ and $C a$ are given in Eq. (2) and Eq. (3), respectively.

$$
\begin{aligned}
& M=\frac{\mu_{p}}{\mu_{d}} \\
& C a=\frac{V_{p} \mu_{p}}{\gamma}
\end{aligned}
$$

where $\mu_{p}$ is viscosity of the penetrating fluid, $\mu_{d}$ is the viscosity of the fluid being displaced, $V_{p}$ is the mean velocity of the penetrating fluid and $\gamma$ is the surface tension.

Depending on the magnitude of these factors, the fluid migration inside the porous medium can take different patterns ranging from compact front to finger-like patterns with varying thickness. In the compact displacement pattern which is indicated by the large square and circular black shapes in Fig. 4.3, the main fluid migration mechanism is cooperative pore filling, in which liquid menisci between neighboring pores overlap and merge into a new stable meniscus. On the other hand, in the finger-like displacement pattern pores are occupied by the fluid in preferential flow paths $[109,110,115]$. 
Furthermore, in finger-like displacement pattern, corner flow in which the penetrating fluid bypasses the pore spaces and advances preferentially along the particle surfaces might take place depending on the affinity of the porous structure to the penetrating fluid $[110,116]$. It has been demonstrated both theoretically and experimentally that higher values of the viscosity ratio $M$ and capillary number $C_{a}$ generally favor the compact displacement [110, 114, 116-120]. In addition, the pore size in the powder bed also significantly influences the imbibition mechanism in the pore scale and consequently fluid-fluid displacement patterns in the larger macroscopic scales, i.e. powder bed scales. In Fig. 4.3, the effect of the pore size on displacement patterns is also shown. In these phase diagrams, large aspect ratio indicates that the pore size is small compared to the average size of channels (pore throat) leading to the pore, and small aspect ratio indicates the opposite scenario where pore size is comparatively larger. While for the fluids with sufficiently large capillary number $C a$, the effect of the pore size can be neglected, for fluids with lower capillary numbers the pore size has significant effect on the displacement patterns.
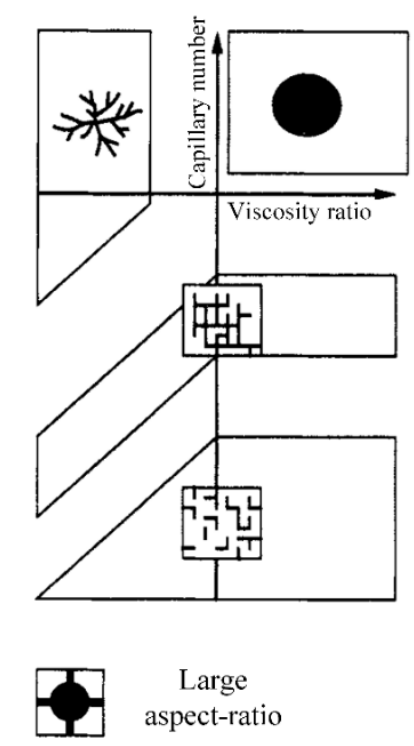

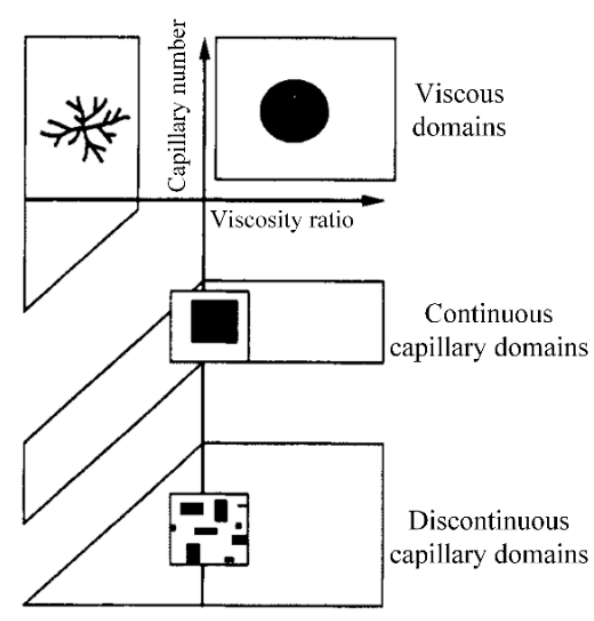

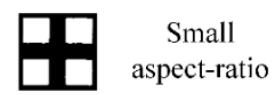

Fig. 4.3 Phase Diagrams for imbibition: a) large aspect ratio; b) small aspect ratio [114] 
In the BJ-AM process, the less viscous air is displaced by a more wetting liquid binder. Therefore, the viscosity ratio $M$ is always favorable for the compact displacement pattern via cooperative pore filling. However, the capillary number $\mathrm{Ca}$ in the BJ-AM process could vary significantly starting from the instant of the droplet impact until the process reaches the equilibrium condition. With the impact of the droplet at the default rate of $8 \mathrm{~m} / \mathrm{s}$ from the system specification of the ExOne M-Lab used in this study, the capillary number $C_{a}$ starts with the value of about 1.5 and becomes zero once the fluid ceases to migrate. As a result, in the BJ-AM process it is expected that the binder imbibition in the powder bed exhibits the transition of penetration patterns. As shown in Fig. 4.4 a, under equilibrium conditions, in the inner regions of the permeation volumes in the powder bed the binder permeation should exhibit the compact pattern regardless of the size of the pores.

On the other hand, towards the boundaries of the permeation volumes where the capillary number $\mathrm{Ca}$ approaches zero, the permeation can exhibit either compact pattern or finger-like patterns depending on the pore size as illustrated in Fig. 4.4 b. For both compact and finger-like patterns, the main displacement mechanism in the pore scale is expected to be cooperative pore filling based on the powder wettability to the liquid binder. For the boundary regions that exhibit finger-like permeation patterns, the resulting finger-like features could also be effectively neglected for the purpose of green part printing due to their lack of structural integrity. Therefore, in the model presented in this study it was assumed that the pores in the powder bed involved in the binder-powder bed interactions are filled completely by the liquid binder. As a result, it can also be assumed that the liquid pressure is transmitted across the droplet volume. Fig. 4.5 clearly exhibits the cooperative 
pore filling for the ether solvent-based binder standard for the ExOne M-Lab system and 420 stainless steel (420SS) powder.

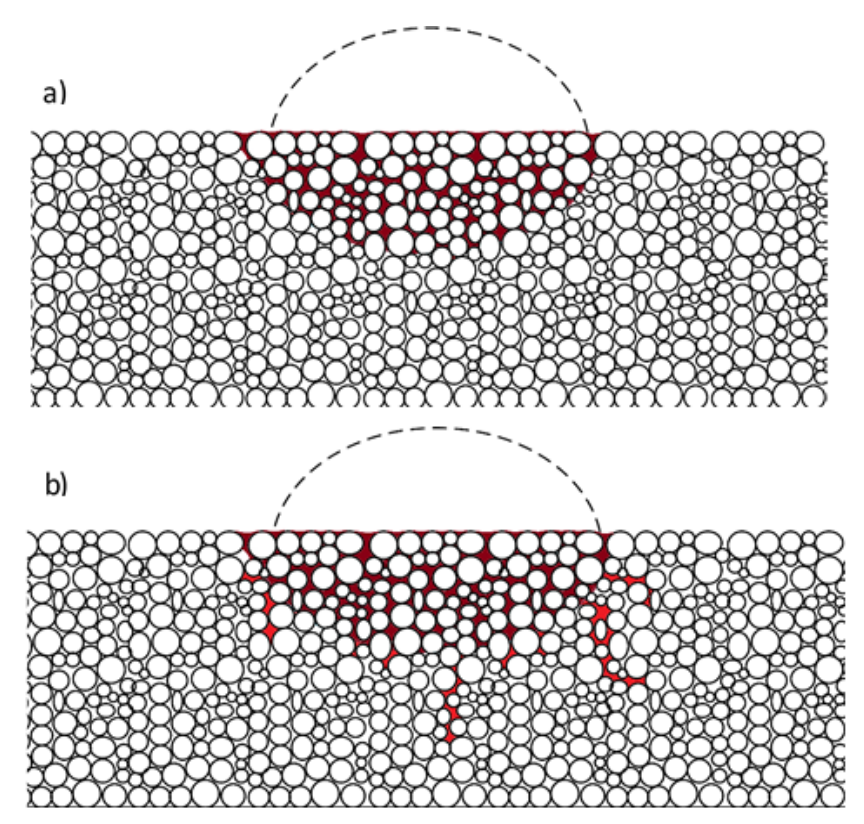

Fig. 4.4 Schematic presentation of a binder droplet pattern inside powder bed (binder droplet after reaching equilibrium state); a) assuming small pore size, b) assuming large pore size

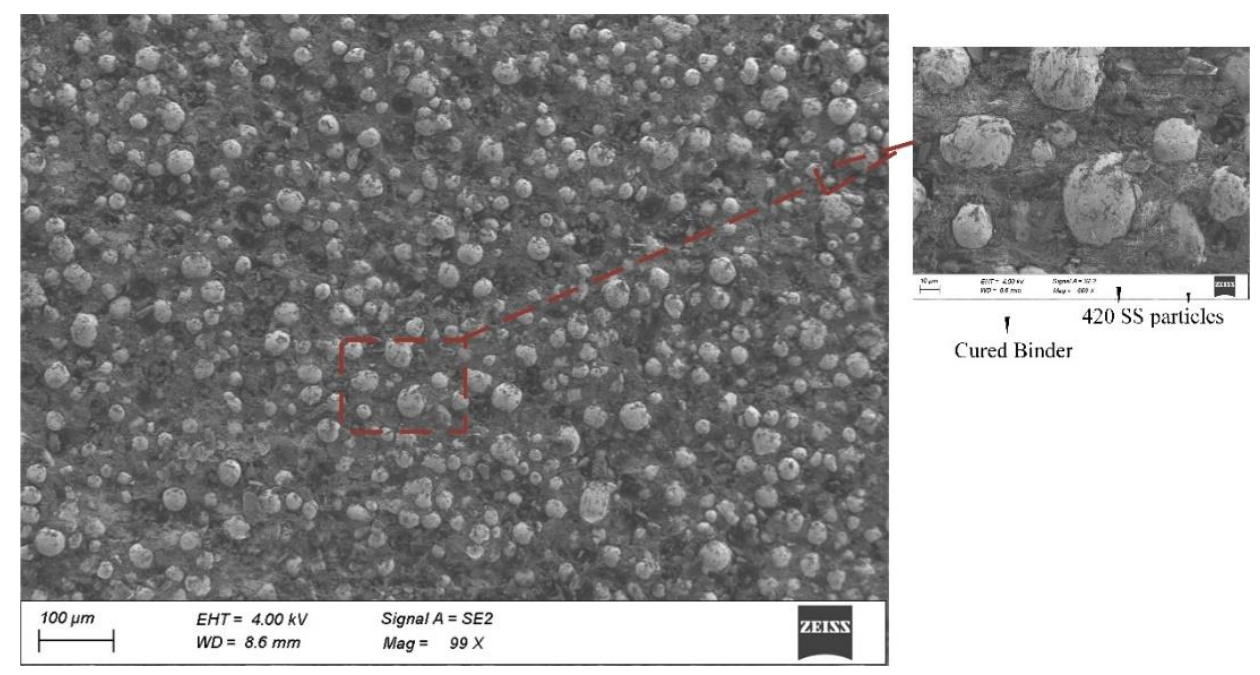

Fig. 4.5 SEM image of binder (deposited by a micropipette) and 420SS interaction in the equilibrium state

After the initial disturbance of the deposited droplet due to the inertial forces is dissipated, it was assumed that the droplet profile will take a semi-spherical shape with the 
instantons radius of $R$. The spherical geometry of the deposited droplet on the surface can be characterized by the dimensionless Bond number, $B$, as $[109,121]$.

$$
B=\frac{\rho g L^{2}}{\gamma}
$$

where $\rho$ is density of the drop, $g$ the gravitational acceleration, $L$ is the diameter of the droplet prior to the impact, and $\gamma$ is the surface tension. While a small $B$ value corresponds to a surface tension-dominated characteristic manifested by a nearly spherical curvature for the droplet, a larger $B$ value implies a weight-dominated characteristic which results in a droplet with a flattened shape $[109,121]$. For the BJ-AM system used in the current study, the resulting Bond number is on the order of $10^{-4}$, which indicates that the spherical curvature is a reasonable approximation.

After the impact, the deposited liquid binder begins to migrate into the surface pores that are in direct contact with the droplet and is gradually absorbed by the powder bed until there is no fluid left on the surface. After complete depletion of the binder droplet from the powder bed surface, binder migration within the powder bed might continue until it reaches the equilibrium conditions where capillary pressures across the binder-air interfaces are balanced out. (Fig. 4.2). The mechanisms considered to drive the binder migration are the capillary pressure and the pressure difference across the interfaces of the penetrating liquid. As a special case of the generalized Young-Laplace relationship, the curved liquid-air interface of the droplet remaining on the surface of the powder bed will cause a pressure difference across the miscues such that the pressure inside the droplet is greater than that of outside (air), which is termed excess pressure in this study [57, 101]. 
With the progression of the binder migration into the powder bed, the excess pressure inside the remaining droplet on the powder bed surface diminishes over time due to the increase of droplet radius from flattening, and eventually becomes zero once the liquid binder is entirely absorbed by the pores inside the powder bed. As a result, the capillary pressure becomes the only significant driving force for migration of the binder liquid after droplet is completely absorbed by the powder bed.

To determine the driving pressure for the binder migration inside the powder bed after the droplet is completely absorbed, the thermodynamic principles for a system consisted of different phases (solid, liquid, and gas) were employed with the assumption of compact advancing of the liquid binder front. Since only the average capillary pressure over the saturated region in the powder bed is of interest in the present study, a cylindrical portion of the droplet profile inside the powder bed as shown in Fig. 4.6 is considered for the estimation of the average driving pressure.

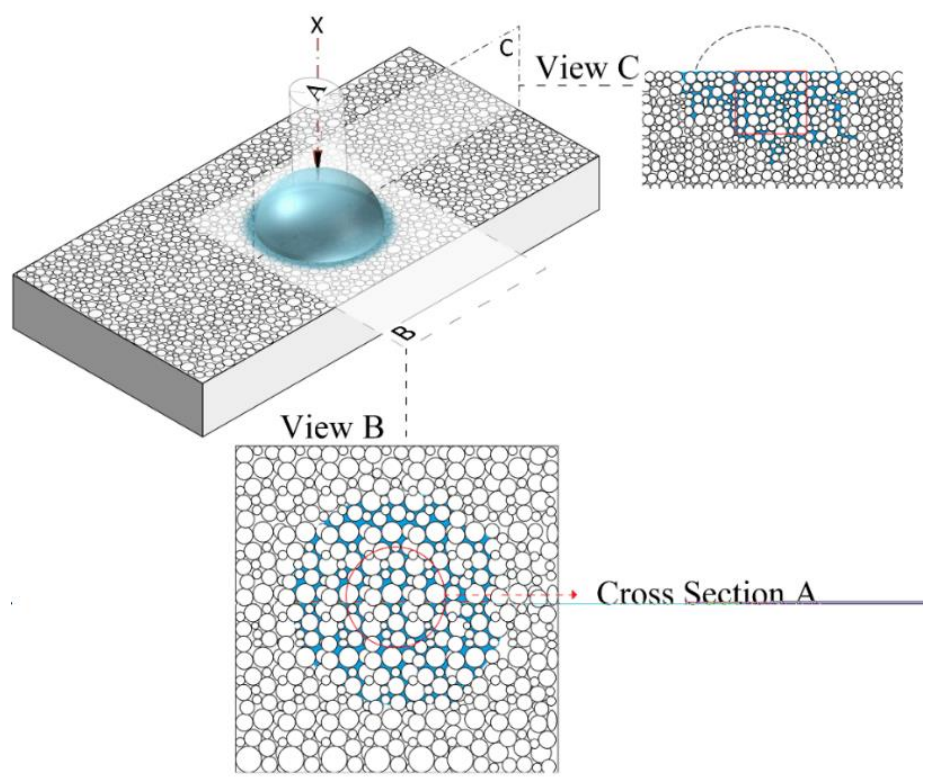

Fig. 4.6 Schematic representation of a droplet penetrating a uniform porous medium 
Within the cylindrical region, the general relationship between force and pressure determines the driving pressure as follows:

$$
P=\frac{F}{A \epsilon}
$$

where $F$ is the driving force, $A$ is the cross section of the selected saturated area in the direction perpendicular to $X$ as shown in Fig. 4.6, and $\epsilon$ is the porosity of the spread powder bed. To determine the force involved in the capillary migration, the general relationship between the force and energy is used according to the equation below.

$$
F=-\frac{d U}{d x}
$$

Where $U$ is the energy of the system including the porous medium and liquid-air interfaces. During the binder permeation in the powder bed, the solid-liquid interfacial area $A_{s l}$, gradually increases, whereas the solid-gas (air) interfacial area $A_{s g}$, gradually decreases. In the current model, due to the cylindrical volume (with constant cross-section A) considered for the analysis the liquid-gas interface can be assumed to remain unchanged over time. Therefore, the variation in the energy of the system can be expressed by

$$
d U=\gamma_{s l} d A_{s l}+\gamma_{s g} d A_{s g}
$$

Where $\gamma_{s l}$ and $\gamma_{s g}$ are the solid-liquid interfacial tension and solid-gas interfacial tension, respectively. Moreover, from the cylindrical geometry the correlation for $d A_{s l}$ and $d A_{s g}$ was obtained as:

$$
\mathrm{d} A_{s l}=-\mathrm{d} A_{s g}
$$

and

$$
\mathrm{d} A_{s l}=S(1-\epsilon) A d x
$$


where $\mathrm{S}$ is the average specific surface area (i.e. surface area/volume) of the powder bed. By substituting the Equations (4.7), (4.8), and (4.9) in Eq. (4.6), the driving force for the binder displacement can be expressed by

$$
F=S(1-\epsilon) A\left(\gamma_{s l}-\gamma_{s g}\right)
$$

As a result, the capillary pressure that drives the binder penetration in the power bed can be written as:

$$
P=\frac{F}{A \epsilon}=\frac{S(1-\epsilon)\left(\gamma_{s l}-\gamma_{s g}\right)}{\epsilon}
$$

Considering the Young equation for the contact angle

$$
\operatorname{Cos} \theta=\frac{\gamma_{s l}-\gamma_{s g}}{\gamma}
$$

Eq. (4.11) can therefore be rewritten as:

$$
P=\frac{S(1-\epsilon) \gamma \cos \theta}{\epsilon}
$$

The pressure $P$ in Eq. (4.13) represents the average pressure that drives the liquid binder migration inside the powder bed when there is no binder left on the powder bed surface. Therefore, under the equilibrium conditions the pressure difference across each binder-air interface in the powder bed pores should follow Eq. (4.13) such that the overall driving pressure gradient becomes zero. Therefore, one can employ Eq. (4.13) to predict the average capillary pressure at equilibrium status, which can be subsequently used to determine the actual equilibrium saturation level with the help of the empirical capillary pressure-saturation curve. 


\subsection{Experimental Procedure}

In this study, two types of powder material, $420 \mathrm{SS}$ and Ti-6Al-4V with different particle size distributions were studied in order to evaluate the effectiveness of the proposed model. The particle size distribution of both powders was characterized by particle size analyzer (Microtrac S3000). The powder particle morphology of both powders was also observed with scanning electron microscopy (Zeiss Supra 35). Fig. 4.7 shows the particle size distribution along with the morphology of both powders. From the results, the 420 SS powder is composed of spherical particles with unimodal size distribution of $35 \mu \mathrm{m}$ average particle diameter. The Ti-6Al-4V powder exhibits bimodal size distribution of $6 \mu \mathrm{m}$ and $32 \mu \mathrm{m}$ average particle sizes. The packing density of the powders spread with the traverse rate of $2 \mathrm{~mm} / \mathrm{s}$ using the roller powder spreading mechanism with the ExOne M-lab system was measured to be $55 \%$ and $66 \%$ for $420 \mathrm{SS}$ and Ti-6Al-4V respectively. In addition, the specific surface area of the powder was determined via Brunauer-Emmett-Teller theory (BET) (TriStar 3000). 

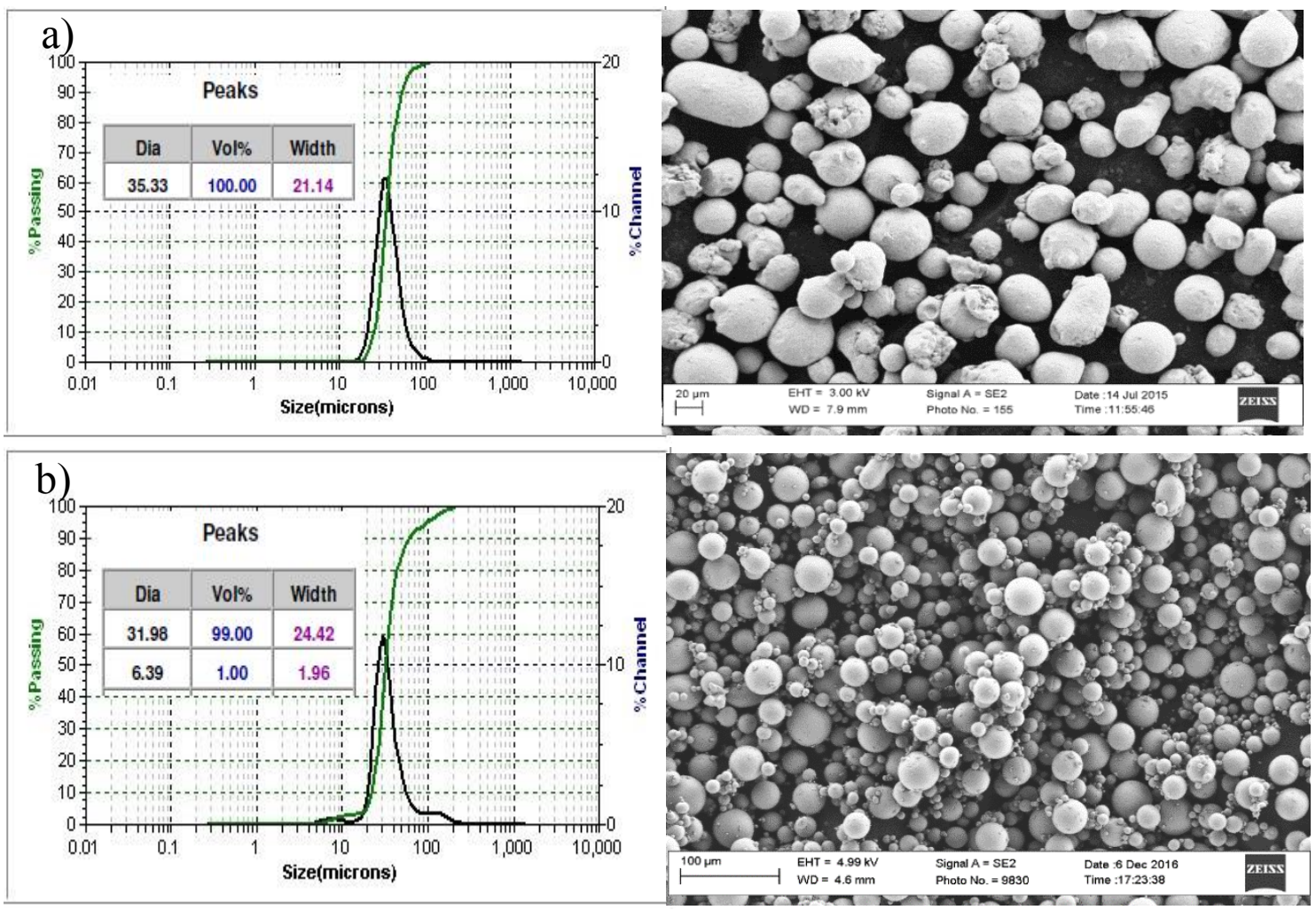

Fig. 4.7 The morphology of a) 420 Stainless steel b) Ti-6Al-4V (grade 23)

Static contact angle is one of the key parameters which can be empirically determined for each powder and liquid binder combination. In this study, the sessile drop method was employed to determine the static contact angle between the liquid binder and different powders $[122,123]$. For this purpose, glass microscope slides $(30 \times 30 \times 2.5 \mathrm{~mm})$ covered with double-sided adhesive tape were shaken briefly in a container of the tested powder, and excess particles which were not firmly attached to the surface were removed by means of low pressure air cleaning $[122,123]$. In order to obtain accurate results, the volumes of the binder droplets used for the contact angle measurements must be chosen in such a way that the effect of gravity can be neglected. When the liquid droplet size exceeds a particular length known as the capillary length as defined in Eq. (4.16), gravitational effects become significant $[124,125]$ : 


$$
l=\sqrt{\frac{\gamma}{\rho g}}
$$

Therefore, considering the appropriate droplet diameter obtained from Eq. (16) for the liquid binder used by the M-Lab, the $2 \mu 1$ droplets were placed on the glass slides, which correspond to $\sim 1.5 \mathrm{~mm}$ droplet diameter that is smaller than the capillary length of the binder $(1.7 \mathrm{~mm})$. Five replicates for each powder material were used for the contact angle measurements, and the average results was used as the contact angle between the binder and the powder material.

To obtain the saturation-capillary characterization curve, a pencil tensiometer was used to directly measure the capillary pressure of the powder beds at different saturation levels. The tensiometer is inserted into the powder bed at the desired saturation level for a certain amount of time, which varies from a few minutes to several hours depending on the level of saturation and powder bed characteristics $[104,126]$. Once the steady-state equilibrium was achieved between the powder material and the measuring system, the reading on the pressure gauge was taken as the capillary pressure of powder bed at the specified saturation level.

Lastly, to determine the experimental equilibrium saturation for the parts fabricated by the M-Lab printer, three sets of features with various dimensions were printed out for each type of the powder materials. The first set of samples (named single-track samples) exhibits line features with cross-sectional dimensions of $0.05 \mathrm{~mm} \times 3 \mathrm{~mm}$ and thickness of $0.1 \mathrm{~mm}$ (one layer). These samples were fabricated in such a way that only one nozzle of the printhead was employed for printing of each track as shown in Fig. 4.8. These printed 
features provide more accurate information about the equilibrium saturation. In the second set of samples (named multiple-track samples), square features of $3 \mathrm{~mm} \times 3 \mathrm{~mm} \times 0.1 \mathrm{~mm}$ were designed and fabricated, which were expected to provide more insights into feature formation in each layer using multiple nozzles. In the third set of samples, cubic features of $8 \mathrm{~mm} \times 8 \mathrm{~mm} \times 8 \mathrm{~mm}$ were designed, which was expected to provide additional knowledge about the overall equilibrium saturation and process of three-dimensional (3D) feature formation. After curing the printed features in a drying oven for 1 hour at $200{ }^{\circ} \mathrm{C}$ following the standard post-printing procedure for the M-Lab green parts, an optical microscope was utilized for measuring the actual dimensions of the printed samples. Subsequently, from the binder volume used for printing the equilibrium saturation in practice was calculated. For all the samples, fabrication parameters of $100 \%$ saturation level, $80 \%$ drying power intensity, 40 s drying time, and $2 \mathrm{~mm} / \mathrm{s}$ spreading speed were employed. The in-process drying parameters, power intensity and drying time, as well as powder spreading speed used in this study were obtained from the preliminary experimentations with the powders. As a general role, greater in-process drying values are needed for higher saturation levels. On the other hand, excessive drying could cause the loss of part integrity by reducing the inter-layer bonding strength. Other parameters used for the green part printing are $100 \mu \mathrm{m}$ layer thickness, $150 \mathrm{~mm} / \mathrm{sec}$ printing speed, and 70 picoliter droplet volume, which are the default setting of the M-Lab system. 


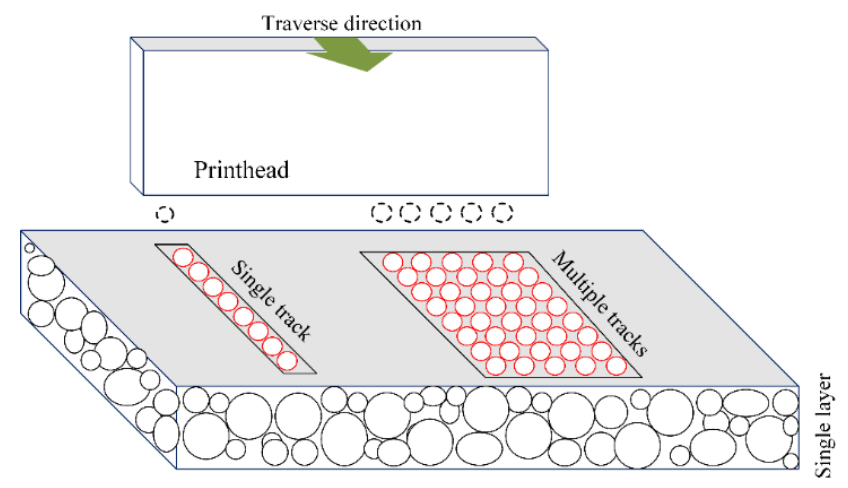

Fig. 4.8 Schematic of single track and multiple tracks experiments

4.4 Results and discussion

Fig. 4.9 illustrates the saturation-capillary pressure characterization curves for both $420 \mathrm{SS}$ and Ti-6Al-4V. As it can be observed from the figure, Ti-6Al-4V powder exhibits relatively higher capillary characteristics compared to 420 SS powder largely due to the smaller pore sizes attributed to smaller particle size and the bimodal size distribution. On the other hand, for the 420 SS powder, the less significant capillary pressure decrease at saturation levels between $5 \%$ and $80 \%$ likely reflects its more uniform pore size distribution in comparison with the Ti-6Al-4V powder [127].

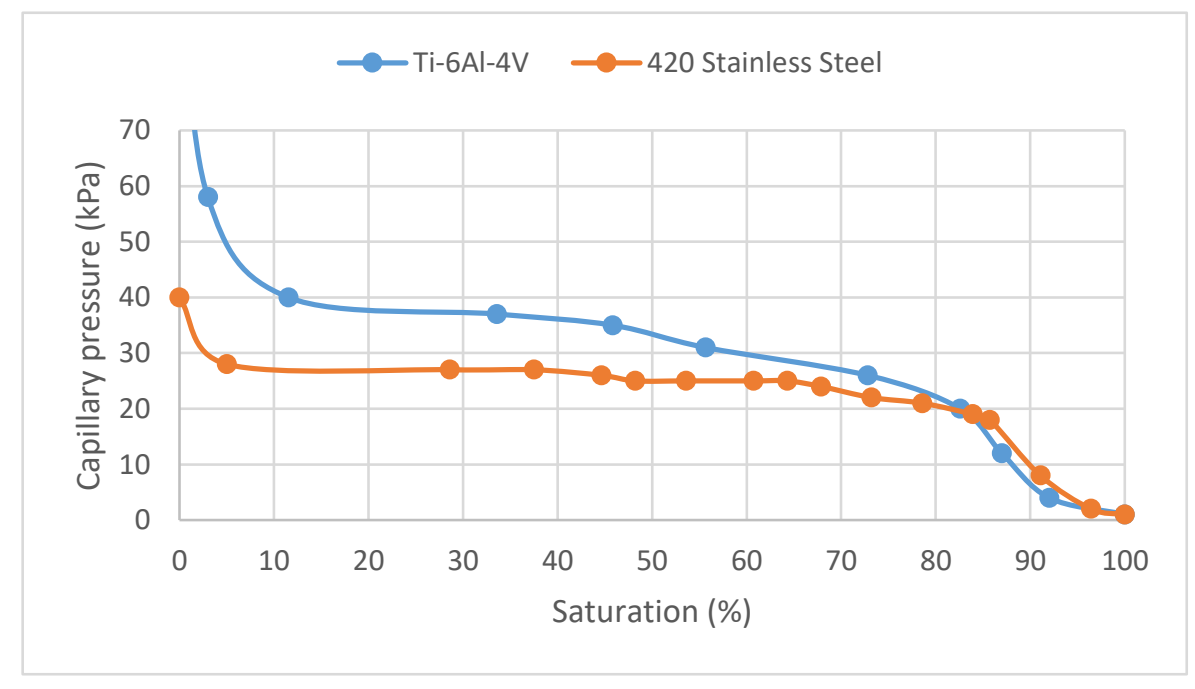

Fig. 4.9 Capillary characteristic curves for Ti-6Al-4V and 420 SS 
The measured results of contact angles and specific surface area from the sessile drop method and BET for both materials are shown in Table 4.1.

Table 4.1 Sessile Drop Method and BET results

\begin{tabular}{|c|c|c|}
\hline Material & Contact angle (degree) & Specific Surface Area from BET $\left(\mathrm{m}^{\cdot 1}\right)$ \\
\hline $420 \mathrm{SS}$ & $25^{\circ}$ & 2 \\
\hline Ti-6Al-4V & $40^{\circ}$ & 0.75 \\
\hline
\end{tabular}

The specific surface area determined by BET corresponds to the total specific surface area of the samples including internal surface area of particles. These internal surface areas include those from the microcracks, microporosities, and microfissures that exist on the powder particle surface, which are accessible by the small gas molecules of $\mathrm{N}_{2}$ commonly used in BET measurement but not binder liquid. As shown in Fig. 4.10, in contrast with Ti-6Al-4V powder, particles of 420 SS possess considerable amount of internal surface areas, which likely contributes to the large specific surface area results from the BET experiment. Therefore, in order to determine the external specific surface area of $420 \mathrm{SS}$ powder that is of interest to the modeling, particle size distribution data from the particle size analyzer (Microtrac S3500) was used with the assumption of spherical particle geometry. Such assumption likely resulted in some underestimation of the specific surface area with the powder. However, with the near-spherical particle morphology observed from SEM, this simplification was considered to be more reasonable approximation than the BET analysis. The external specific surface area obtained from the size distribution analysis, $0.2 \mathrm{~m}^{-1}$, which differed significantly from the BET measurement 
results of $2 \mathrm{~m}^{-1}$, suggesting that there exists considerable amount of internal surface area on the 420 SS particles.
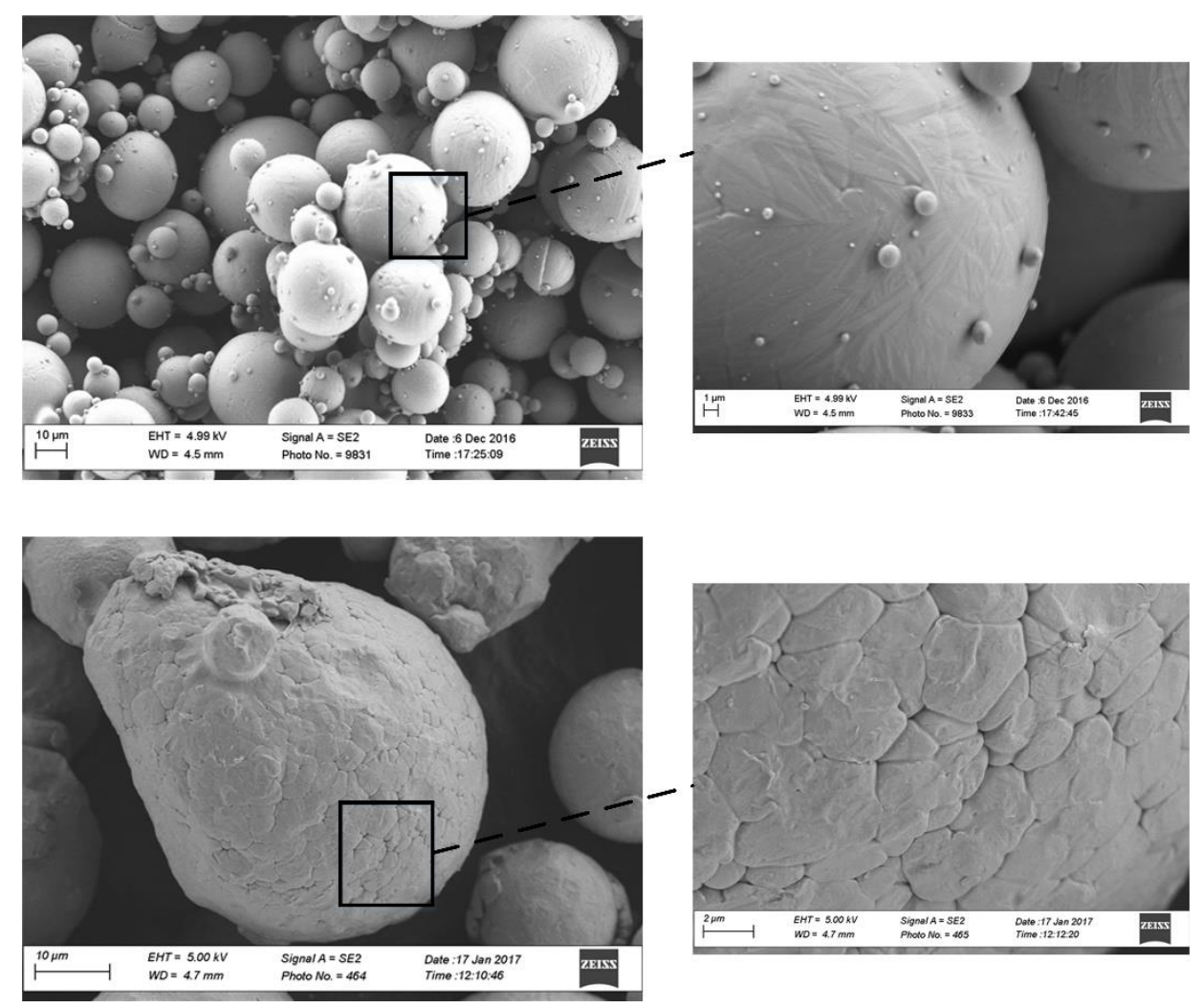

Fig. 4.10 SEM micrograph of powder materials used, a) Ti-6Al-4V b) $420 \mathrm{SS}$

Powder wettability to the invading liquid is one of the critical parameters which would significantly influence the binder distribution within the powder bed as well as the saturation levels in the equilibrium state. From both Young-Laplace relationship and the proposed model, greater affinity of the powder material to the binder fluid that is described by low contact angle corresponds to the increase of the capillary pressure levels under the equilibrium conditions. Therefore, from the contact angle measurement results, both materials investigated in this study show similar affinity to the liquid binder used. In addition, the binder surface tension and binder density were provided by the manufacturer (ExOne). 
Based on the experimental results, the capillary pressure in the equilibrium condition for each type of powder materials can be estimated from the proposed model as follows:

420 SS powder

$$
P=\frac{S(1-\epsilon) \gamma \cos \theta}{\epsilon}=\frac{0.2 \times 10^{6} \times 0.55 \times 0.03 \times \cos 25}{0.45}=7 \mathrm{KPa}
$$

Ti-6Al-4V powder

$$
P=\frac{S(1-\epsilon) \gamma \cos \theta}{\epsilon}=\frac{0.75 \times 10^{6} \times 0.66 \times 0.03 \times \cos 40}{0.34}=33 \mathrm{KPa}
$$

From the model, the capillary characteristic of the Ti-6Al-4V powder under equilibrium condition is remarkably greater than that of the $420 \mathrm{SS}$ powder. For both materials studied in this study, the two most significant factors for the equilibrium capillary pressure are the specific surface area and packing density of the powders. From the literature, it is well known that in general smaller pore size corresponds to higher packing density, which in turn indicates higher capillary pressure of powder material, since an equivalent capillary tube with a smaller diameter would exhibit greater capillary pressure according to the Young-Laplace relationship [53, 57, 101, 104]. On the other hand, the total energy of such systems in which two or more phases are under equilibrium conditions is strongly dependent on the surface area of the phases involved in the interaction as discussed previously.

The estimated equilibrium quantities, $7 \mathrm{kPa}$ and $33 \mathrm{kPa}$, for capillary pressures correspond to $92 \%$ and $50 \%$ saturation levels for 420 Stainless Steel and Ti-6Al-4V powder materials respectively according the capillary-saturation characterization curves. 
The samples for experimental equilibrium saturation evaluation are shown in Fig. 4.11. The equilibrium saturation level measured from single track features are $53 \%$ and $60 \%$ for Ti-6Al-4V and $420 \mathrm{SS}$ materials, respectively. From the multiple-track experiments, similar values with slight variations ( $57 \%$ for Ti- $6 \mathrm{Al}-4 \mathrm{~V}$ powder and $66 \%$ for 420 SS material) were obtained for both materials. However, the measurements with the cubic parts for both materials exhibit significantly greater levels of equilibrium saturation compared to single and multiple-track features. For the cubic samples printed with Ti-6Al$4 \mathrm{~V}$ powder, the equilibrium saturation level was measured to be $82 \%$, and similarly, for the 420 SS powder the value was obtained to be $86 \%$.

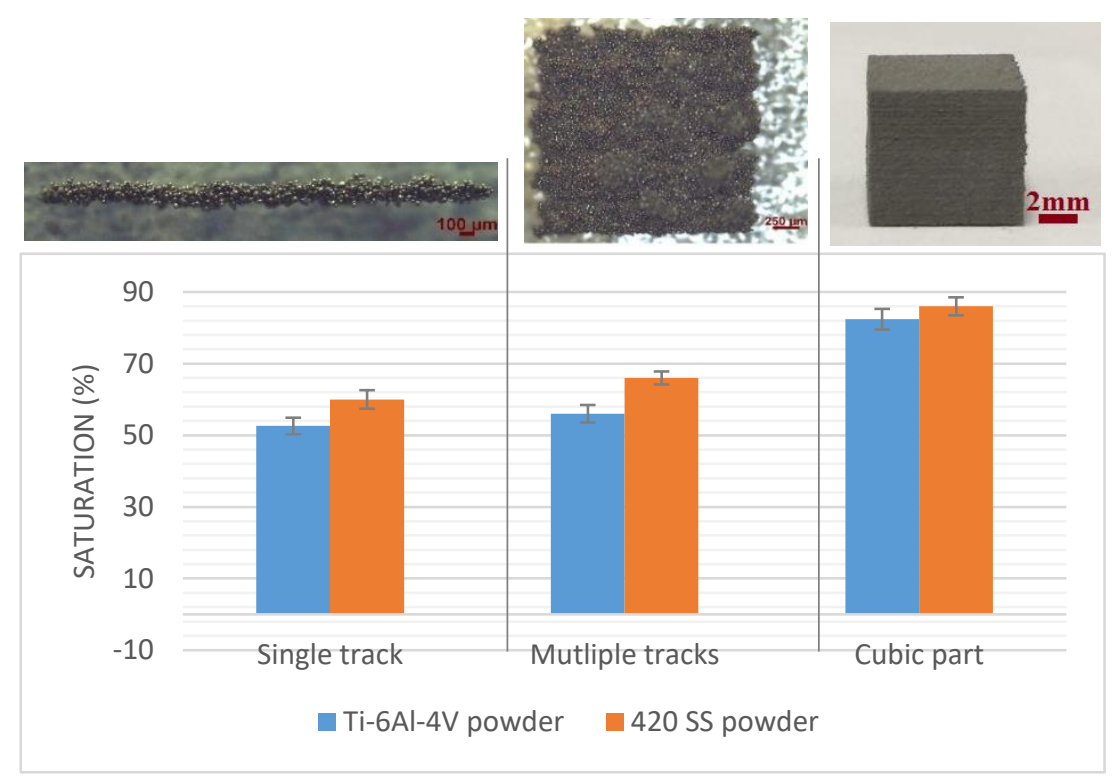

\section{Fig. 4.11 Equilibrium saturation. The error bars indicate the standard deviation for each set of experiments.}

It was observed from single and multiple track experiments that the deviations between the experimental equilibrium saturation and the theoretical saturation were to a large extent caused by the dimensional inaccuracy of the thickness of printed features, which is perpendicular to the powder bed surface. The dimensional inaccuracies of the 
printed features arise largely from feature thickness, which is associated with the depth of binder penetration in the thickness direction rather than its lateral spreading in the directions perpendicular to the penetration direction. It was found that the penetration rate of the liquid binder within the powder bed appears to be greater than the spreading rate. Such nonuniformity in the fluid migration rate in the vertical and lateral directions depends on many factors including the binder and the powder material properties, powder bed characteristics, and powder wettability. For the cubic parts which consist of multiple layers, two possible mechanisms of droplet dynamics within the powder bed might contribute to the feature formation and the saturation level deviations compared to the single layer samples. In the first mechanism, the liquid binder in the previously generated layers underneath the layer being printed is sufficiently dried. As a result, the penetration of the binder within the layer in the vertical direction is restricted by the layers beneath it, which causes enhanced saturation in the printed areas as well as more significant binder spreading toward the unsaturated area between the deposited droplets as shown schematically in Fig. $4.12 \mathrm{a}$, which consequently results in higher saturation levels. In the second mechanism where the in-process heating parameters are not optimally chosen to sufficiently dry the binder, the deposited liquid binder in the top layer end up supplying excessive binder to underneath layers. In addition, under the second mechanism, the residual binder will be driven to penetrate into previous layers under the external pressure due to the weight accumulation of the printed structures, which consequently decrease the dimensional accuracy of the printed features as illustrated in Fig. 4.12 b. It is clearly shown in Fig. 4.12 that for the two printed samples with different in-process heating parameters, the 
dimensional error of the part fabricated under insufficient drying settings is significant due to the excessive saturation levels towards the bottom layers.

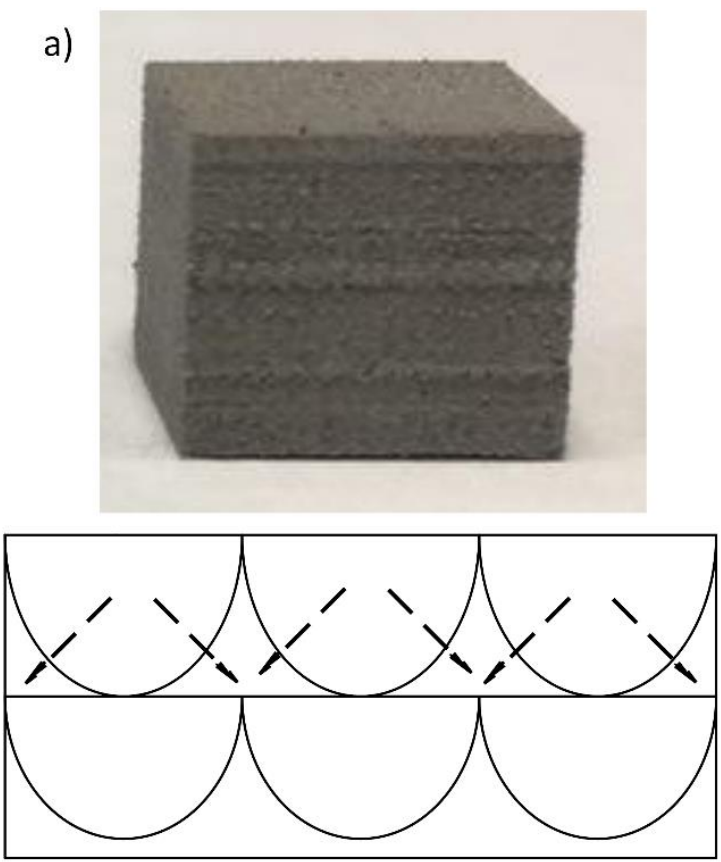

Well dryed binder b)

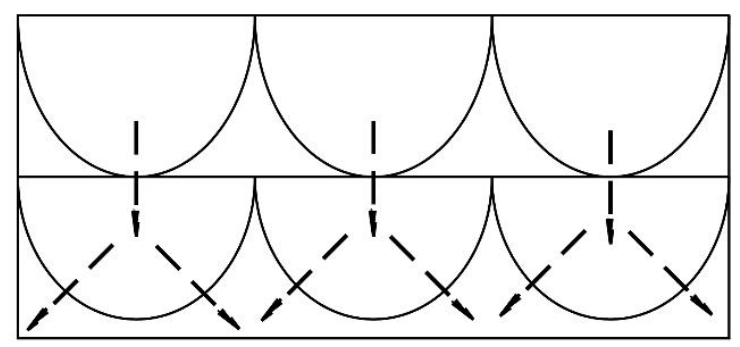

Insufficiently dryed binder

Fig. 4.12 schematic of parts printed with different in-process binder curing parameters a) insufficient drying parameters b) appropriate drying parameters (the dashed arrows indicate the potential binder migration paths inside the powder bed)

Comparing the experimental results of equilibrium saturation measurements from the single and multiple tracks with the theoretical predictions, overall there exists a good agreement for Ti-6Al-4V powder material. However, for 420 SS powder the theoretical prediction for equilibrium saturation is considerably higher than the experimental results from single and multiple tracks parts. This discrepancy for 420 SS powder can be largely attributed to the existence of the microscopic surface features on the powder particle surfaces which are mostly not accessible by the liquid binder. The results from BET method and particle size analyzer provide two extremes for the specific surface area involved in the interaction. As the lower bound value from the particle size analyzer was used for the equilibrium saturation predictions, the resulting capillary pressure was likely 
underestimated, which corresponds to the higher amount of saturation in the equilibrium state.

Furthermore, the $50 \%$ saturation level predicted from the model for the Ti-6Al-4V powder was implemented for printing a set of cubic samples to evaluate the effectiveness of the model prediction in term of integrity and quality of the parts. Observations from empirical measurements indicated that fabricating parts with optimal saturation level results in better dimensional accuracy. As shown in Table 4.2, the comparison of the measured dimensions for the cubic Ti-6Al-4V samples with nominal sizes of $8 \mathrm{~mm} \times 8 \mathrm{~mm}$ $\mathrm{x} 8 \mathrm{~mm}$ clearly show better overall accuracies for the sample fabricated with $50 \%$ saturation levels, which shows that the model could provide accurate predictions of the optimal printing saturation levels that are in good agreement with the experimental-based estimations $(50 \%$ theoretical prediction vs. $49 \%$ experimental results). Therefore, the model for the equilibrium saturation established in the current study appears to be able to provide accurate estimation of the optimal saturation value for printing that results in optimized dimensional accuracy. Considering the definition of equilibrium saturation, the new model essentially predicts the least amount of liquid binder that is required for feature fabrication via BJ-AM process without the loss of integrity. Although higher saturation levels might be used for printing purpose with appropriate adjustments of in-process heating parameters, the excess amount of binder will often result in decreased dimensional accuracy due to the binder permeation from the designated boundaries and the higher possibility of binder residual in the microstructure after the sintering. It is noted that the 1D equilibrium saturation experiments with single tracks yielded effective predictions of optimal saturation levels for the 3D structures, which implies that the assumption of 
compact permeation pattern is likely a reasonable approximation of the permeation boundaries. Since this type of binder is expected to have good wettability to metal powder in general, such results also suggests that for high quality metal powder with good flowability, the characterization approach described in the current study could be applied for rapid development of optimal green part printing parameters.

Table 4.2 Effect of optimal saturation level on the dimensional accuracy of Ti-6Al$4 \mathrm{~V}$ samples

\begin{tabular}{|l|l|l|l|}
\hline Saturation & $\mathrm{X}(\mathrm{mm})$ & $\mathrm{Y}(\mathrm{mm})$ & $\mathrm{Z}(\mathrm{mm})$ \\
\hline $50 \%$ & 8.04 & 8.06 & 8.00 \\
\hline $100 \%$ & 8.12 & 8.16 & 8.20 \\
\hline
\end{tabular}

4.5 Conclusions

In the BJ-AM process, the binder saturation is of critical significance in determining the printed part quality. Insights into appropriate binder saturation for specific liquid binder and powder bed interaction are desired for the rapid development of new materials for high part integrity and dimensional accuracies. In the current study, a new physics-based model was developed to estimate the optimum saturation amount required during the feature fabrication in BJ-AM process. This model establishes the capillary pressure conditions under equilibrium saturation, which was consequently calibrated by experimental capillary pressure-saturation curves. It was demonstrated that the permeation of the BJ-AM green part printing process is largely driven by both the excess pressure and the capillary pressure, and the equilibrium conditions of the binder permeation is predominantly influenced by the capillary pressure. This model was applied to the optimal saturation 
predictions of two types of powder materials, Ti-6Al-4V and $420 \mathrm{SS}$. The saturation analysis for single-track, multiple-track, and cubic features with various dimensions were carried out, and the results showed that there exists a good agreement for Ti-6Al-4V material between the theoretical predictions and the experimental data. However, for the 420 SS powder, due to the existence of internal microscopic surface areas that do not contribute to the wetting of the powder, the binder-powder interactions could not be adequately accounted for by the total surface areas of the powder, which would result in overestimation of the optimal saturation levels. As the accurate estimations of the optimal saturation levels could result in not only better green part printing robustness but also improved geometrical accuracy, the proposed model is of importance for the efficient development of the BJ-AM processes for optimal green part qualities. 


\section{CHAPTER 5}

\section{EFFECT OF PRINTING SPEED ON QUALITY OF PRINTED PARTS IN BINDER JETTING PROCESS}

\subsection{Introduction}

Binder jetting additive manufacturing process (BJ-AM) is an additive manufacturing process in which a part geometry is fabricated by applying liquid binder to the selective areas of a spread layer of powder material. In this process, small droplets with diameters less than $100 \mu \mathrm{m}$ are successively deposited onto the powder bed surface through a drop-on-demand (DOD) printhead in a pattern of raster scanning. After deposition of the liquid binder, the entire surface of the powder bed, including saturated and unsaturated areas, is exposed to a fixed amount of heat commonly by means of a heat lamp. This inprocess heating is to establish appropriate mechanical strength via partially cured binder within the already generated layer to withstand the shear and gravitational compressive forces involved in the spreading and printing of subsequent layers. These steps are repeated for each layer until the whole feature is completed [100]. The quality and integrity of the components printed via BJ-AM process might be significantly affected by the physical properties of the liquid binder, powder material, powder bed characteristics and process parameters. Some of these process control parameters, including saturation level, heating 
power intensity, liquid binder curing time, feed-to-powder ratio, and spreading speed, have been experimentally investigated for their effect on the dimensional accuracy and mechanical strength of the printed features $[8,11,14,26,28,98,105]$. The saturation level, binder amount used for part printing, coupled with binder curing parameters (power intensity and curing time) has been shown to play a key role in determining the quality of the fabricated parts $[26,29,103,128]$. While the excessive binding agent would result in dimensional inaccuracies, insufficient usage of binder would deteriorate the mechanical performance of the printed components [25]. In [55], the authors have developed a physicsbased model to predict the optimal saturation amount which ensures the structural integrity and dimensional accuracy of the printed features. The quality of the fabricated parts may also be influenced by the powder spreading speed and the feed-to-powder ratio (thickness of feed layer to layer thickness that is attributed to the change in powder packing characteristics and spreading uniformity $[14,129]$. The effect of layer thickness has also been evaluated by different researchers $[11,25,98]$. The layer thickness which is used for binder saturation calculations might remarkably affect the dimensional accuracy and mechanical strength of the printed features [25, 26, 29]. With a fixed binder saturation and droplet volume, thicker layers would require further deposition of binder in the already pre-wetted area which might consequently influence the binder flow dynamics. It is suggested that for the desirable spreading of powders in BJ-AM process, the layer thickness should be at least thicker than the largest particle and preferably three times of particle size [130]. On the other hand, the printing speed, which corresponds to the forward travel rate of the printhead (in Y direction as shown in Fig. 5.1), has received little attention in the literature. While it is generally perceived that for AM higher printing speed would 
correspond to reduced geometrical accuracy of the printed parts, the driving mechanisms differ among processes. For example, in powder bed fusion the printing speed could be conveniently interpreted as the moving speed of the melting pool, which impacts the printing quality by imposing different spatiotemporal thermal characteristics [131, 132]. Similarly, in vat photopolymerization the printing speed affects the curing characteristics of the parts, which in turn affects the dimensional accuracy of the parts [133, 134]. In material jetting additive manufacturing process, the printing speed effect on part quality would be manifested in surface characteristics of the printed features $[135,136]$. However, for BJ-AM technology such fundamental understanding of mechanisms and their impacts on quality of the fabricated components have not been widely studied.

Similar to the interaction of a droplet with an impermeable surface, three phenomena, spreading, bouncing (i.e. rebounding of impinging droplets on a solid surface) or splashing, might take place upon impact of a droplet on a porous surface. Such behaviors between an impinging droplet and a permeable surface depend on many factors including the physical properties of the droplet and the target surface, compact conditions (impact velocity and droplet size), porous structure characteristics, and the wettability of the porous material to the droplet [137-139]. One of the key parameters determining the droplet interaction with the any surface is the velocity of the impinging droplet [139-141]. It has been shown both experimentally and analytically that the velocity of the droplets upon impact has a significant influence on droplet flow dynamics in porous media due to effect of the inertia forces $[113,138,139,142-145]$. While spreading dominates the interaction of the impinging droplet and the surface at low velocities, bouncing and/or splashing are the dominant mechanisms in the interaction at high impact velocities. In BJ-AM process, 
as the formation of green parts is closely associated with the binder permeation characteristics in the porous powder bed, occurrence of each of these phenomena primarily due to the factors such as initial droplet shape and velocity vector, powder bed pore characteristics and binder liquid characteristics could significantly impact not only the equilibrium binder saturation conditions but also the geometry of the binder-wetted area. Spreading due to the droplet impact would increase the initial contact area between the droplet and powder bed and as a result would influence the subsequent droplet penetration and saturated area. On the other hand, if bouncing phenomenon occurs, the impinging droplets would rebound from the powder bed surface upon initial contact, which could potentially result in offset of permeation zone along the $\mathrm{Y}$ direction from the initial location and introduce dimensional inaccuracies to the printed structures. Also, splashing in which the impinging droplet disintegrates in two or more secondary smaller droplets after impact might contribute to loss of integrity and accuracy of parts by decreasing the droplet permeation area. Therefore, it is expected that the printing speed as the horizontal component of the overall binder droplet velocity (Fig. 5.6) could significantly affect the geometrical accuracy and saturation characteristics of the green parts, which was experimentally investigated in the present study.

\subsection{Methodology}

To assess the effect of printing speed on components fabricated via BJ-AM process, cuboid samples with $8 \times 8 \times 2 \mathrm{~mm}$ dimensions which contain narrow slots with width of $0.25 \mathrm{~mm}$ were fabricated using ExOne M-Lab printer. As shown in Fig. 5.1 The designed geometry for printing samples and part configuration in the powder beda set of such 
samples was fabricated in such a way that slots were aligned with the printing direction ( $\mathrm{Y}$ direction). For fabrication of the second group of these structures, parts were oriented along with $\mathrm{X}$ direction (perpendicular to printing direction). The narrow slots in the design as shown in Fig. 5.1 were designed to capture the effect of the printhead speed on dimensional accuracy in $\mathrm{Y}$ and $\mathrm{X}$ directions. A broad range of printing speeds from $15 \mathrm{~mm} / \mathrm{sec}$ to 1000 $\mathrm{mm} / \mathrm{s}$ were applied for the fabrication of the parts.

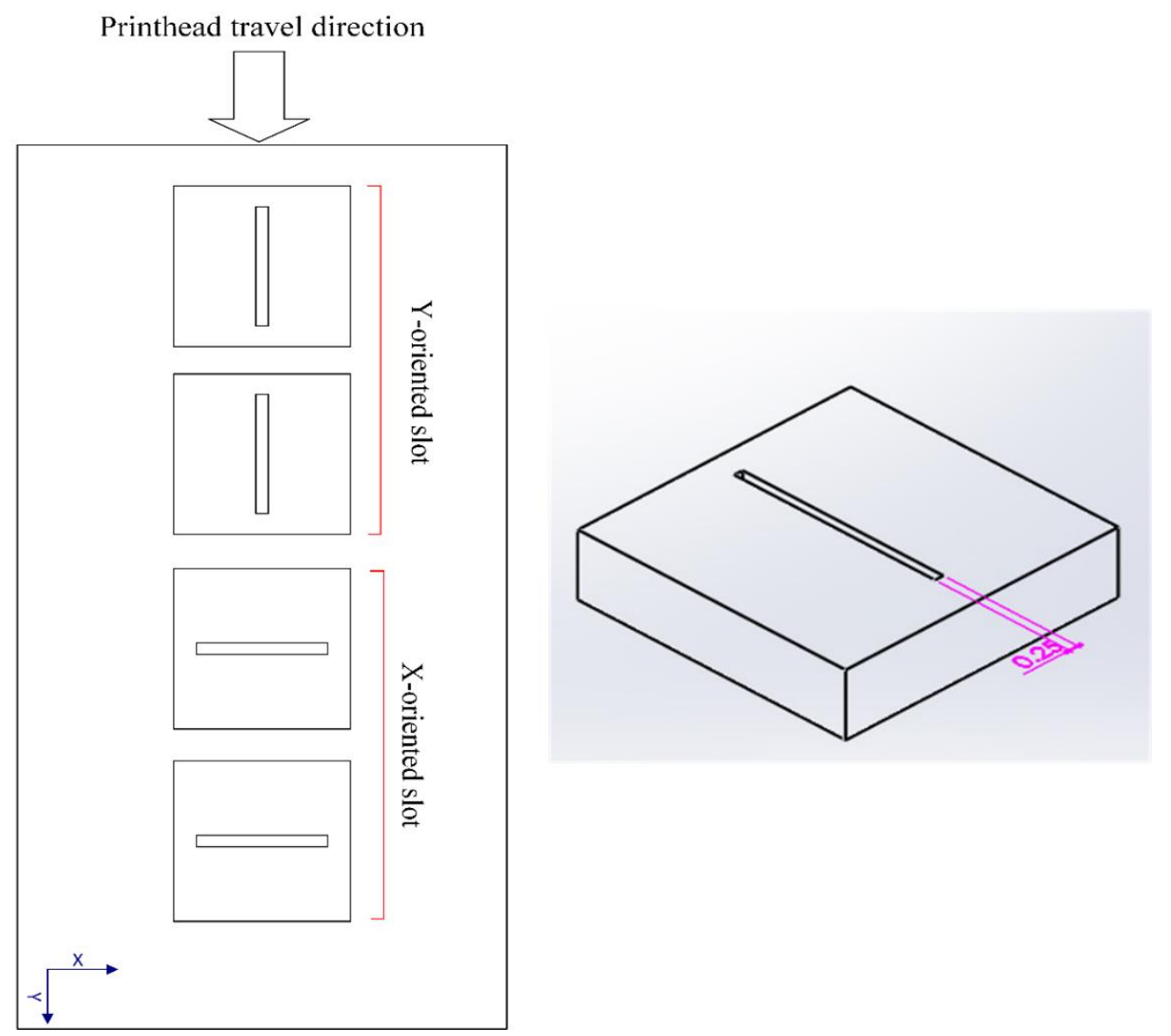

Fig. 5.1 The designed geometry for printing samples and part configuration in the powder bed

In order to evaluate the bouncing phenomenon, a series of cubic samples were fabricated in such a way that printing speed was adjusted for every 10 layers of printing from 100 to $300 \mathrm{~mm} / \mathrm{s}$. 
Lastly, three sets of features with various dimensions were fabricated in an attempt to quantify the effect of printing speed on dimensional accuracy and saturation measure of printed samples. The first set of samples (single-track samples) were straight line features with cross-sectional dimensions of $0.05 \mathrm{~mm} \times 3 \mathrm{~mm}$ and thickness of $0.1 \mathrm{~mm}$ (one layer). The dimensions of these feature were selected in a way that only one nozzle of the printhead was employed for printing of each track (line) as shown in Fig. 5.2. These printed features are intended to provide more accurate information about the effect of printing speed on dimensional accuracy of the printed parts without introducing potential influences of other process parameters such as printing pattern and spacing between successive droplet deposition. For the second set of samples (named multiple-track samples), square features of $3 \mathrm{~mm} \times 3 \mathrm{~mm} \times 0.1 \mathrm{~mm}$ were designed and fabricated, which give more insights into feature formation and printing speed effect on single-layer structure using multiple nozzles. In the third set of samples, cubic features of $3 \mathrm{~mm} \times 3 \mathrm{~mm} \times 3 \mathrm{~mm}$ were designed, which were expected to provide useful knowledge about the overall printing speed influence on quality and integrity of the printed 3D structures. The equilibrium saturation of the printed cubic samples was also experimentally determined to examine the influence of the printing speed on the equilibrium saturation of printed features in practice. The saturation level was considered to be the ratio of the total binder volume used for fabrication by the printer and the total volume of the void phase of a cubic part which is determined by knowing the packing density and the measured volume of the sample (measured volume of a sample * (1-packing density)). The total binder volume can be determined using the binder saturation level employed for printing and the nominal volume of the printed features. The average binder volume jetted from each nozzle of the printhead is pre-calibrated before fabrication 
process. Knowing the desired saturation level and the part nominal dimensions, it is possible to obtain the total binder volume that is used for fabrication of each part [55].

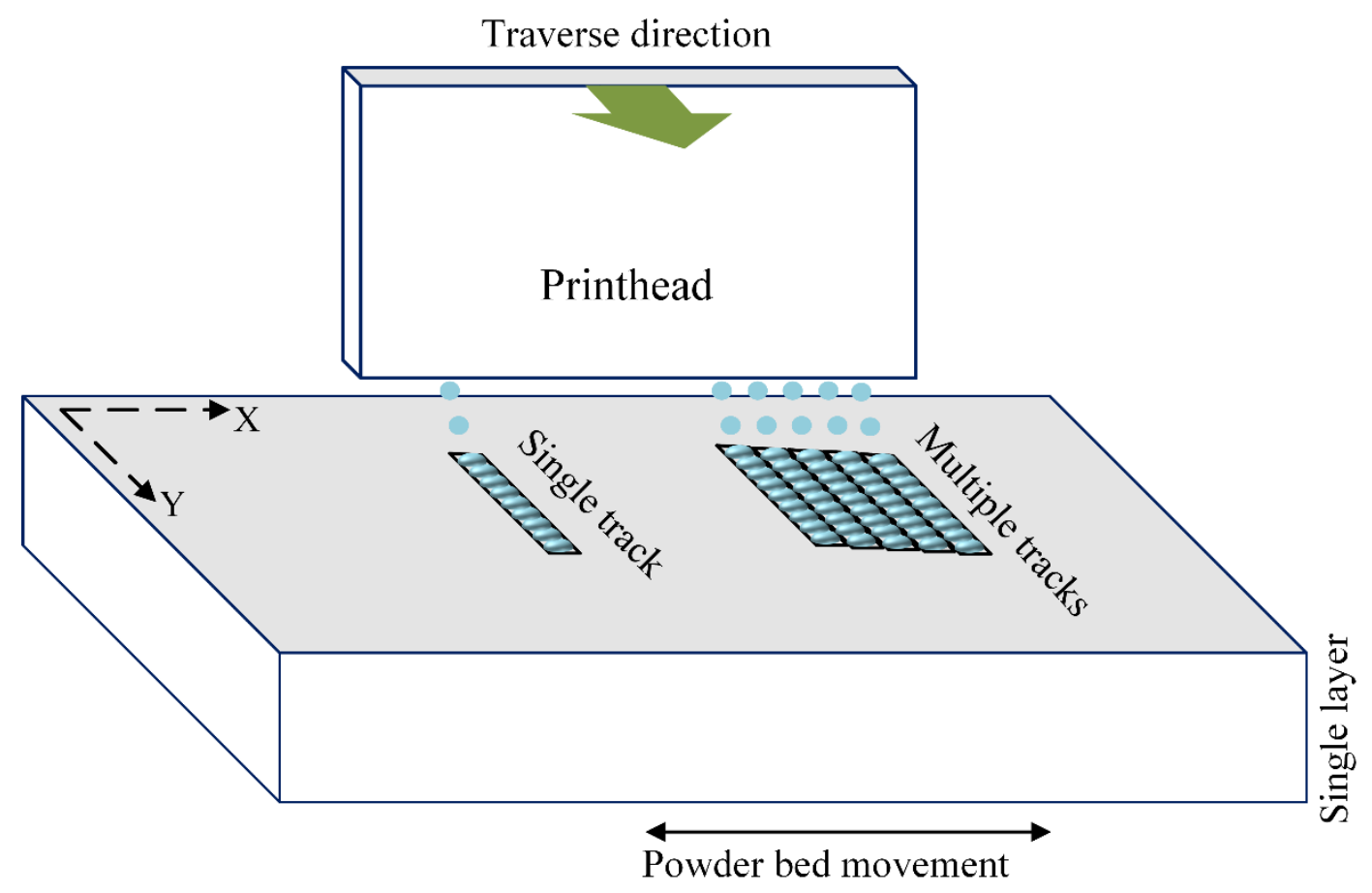

Fig. 5.2 Schematic of single track and multiple tracks experiments

420 Stainless Steel (ExOne LLC) was used for fabrication of samples. The powder was characterized via particle size analyzer, and Fig. 5.3 shows the particle size distribution along with the morphology of the powder. The results showed that the 420 SS powder is composed of spherical particles with unimodal size distribution of $35 \mu \mathrm{m}$ average particle diameter. The process parameters used for printing the samples including saturation level $(100 \%)$, curing power intensity (90\%), curing time (45 sec), layer thickness $(100 \mu \mathrm{m})$ and spreading speed $(2 \mathrm{~mm} / \mathrm{sec})$ were kept constant for all experiments. After the samples were printed, they were placed in an oven and cured at $180^{\circ} \mathrm{C}$ for 1 hour for curing. In addition, the packing density of the powder material once spread with the traverse rate of $2 \mathrm{~mm} / \mathrm{sec}$ using ExOne M-lab printer was measured to be $55 \%$. For measuring the packing density, 
a cylindrical container with outer diameter of $35 \mathrm{~mm}$, height of 15 , and $3 \mathrm{~mm}$ wall thickness was printed. After curing, the weight of the powder in the container as well as the container pocket volume was measured, which were consequently used to calculate the powder bed density. The packing density was calculated by dividing the powder bed density by the powder material solid density. An optical microscope (Olympus MX51 with Olympus QColor 5 digital camera) was utilized for measuring the width of slots and dimensional accuracy of all the printed features including single-track and multi-track features.

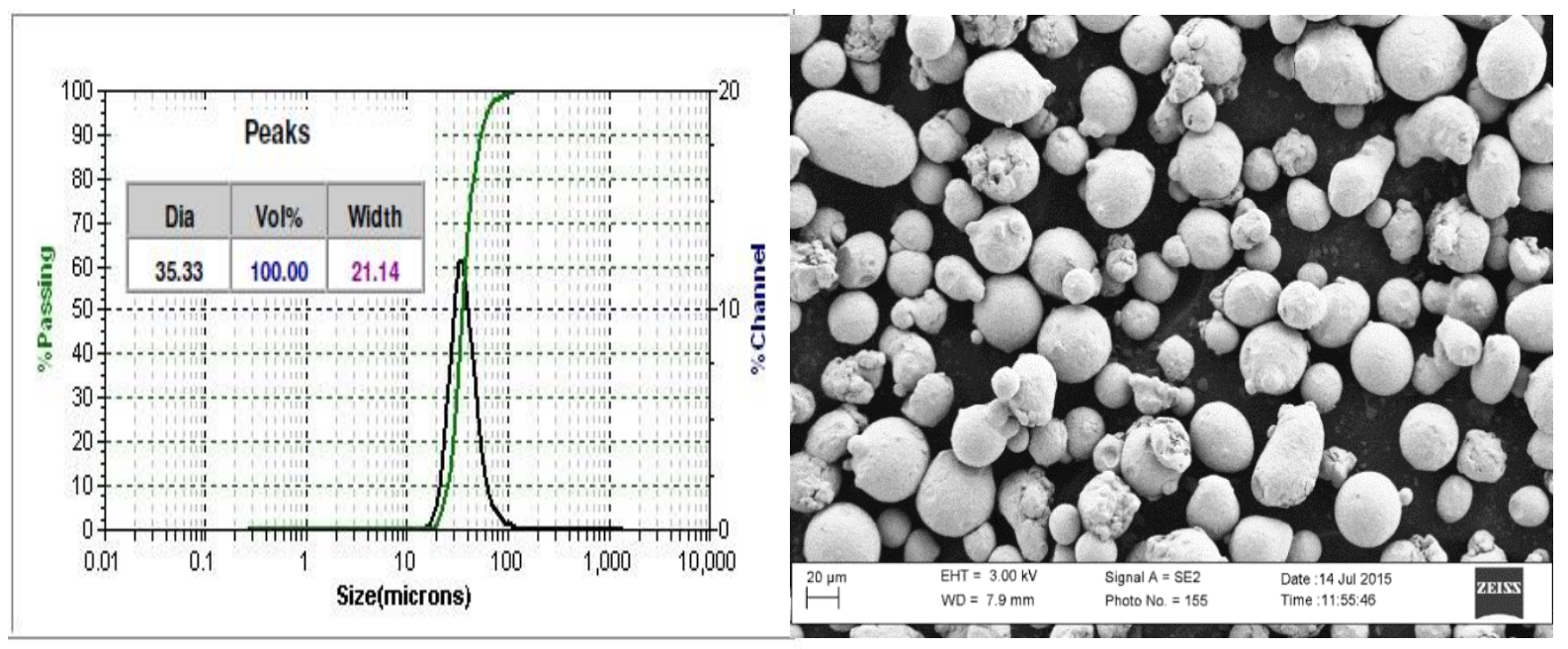

Fig. 5.3 The morphology of 420 Stainless Steel powder material

In order to measure the wettability of the powder material to the liquid binder, the sessile drop method was employed to determine the static contact angle between the liquid binder and $420 \mathrm{SS}$ powder. For this purpose, glass microscope slides $(30 \times 30 \times 2.5 \mathrm{~mm})$ covered with double-sided adhesive tape were shaken briefly in a container of the tested powder, and excess particles which were not firmly attached to the surface were removed by means of low pressure air cleaning. The $2 \mu 1$ droplets were placed on the glass slides and the contact angle was measured by an optical microscope. Five replicates were used for the contact angle experiment, and the average results was used as the contact angle 
between the binder and the powder material $[122,123]$. More details about the contact angle measurement can be found in [55].

\subsection{Results and discussion}

Fig. 5.4 exhibits optical microscopy $(\mathrm{OM})$ images of the parts fabricated by various printing speeds with close up views of the slots printed with $300 \mathrm{~mm} / \mathrm{sec}$. From the figure, it is clear that the printing speed affects the dimensional accuracy of both sets of the printed slot features. Although increasing the printing speed decreases the accuracy of the intended slots in the printed parts for both orientations, it seems that the slots in X-oriented features are influenced more significantly by the printing speed than the Y-oriented ones. Quantitative dimensional comparison of printed features in terms of slot width is shown in Fig. 5.5. For all samples fabricated with different printing speeds, accuracy of Y-oriented features is greater that than of X-oriented features. It can be observed from the figure that this discrepancy between the accuracy of differently oriented features increases with the printing speed. The feature fabricated with the lowest printing speed, $15 \mathrm{~mm} / \mathrm{sec}$, has the highest accuracy and smallest discrepancy between the orientations. With high printing speeds ( $>400 \mathrm{~mm} / \mathrm{s})$, while the X-oriented slot features become unrecognizable from the printed samples, in Y-oriented parts the slots are still clearly present albeit with lowest accuracy. 


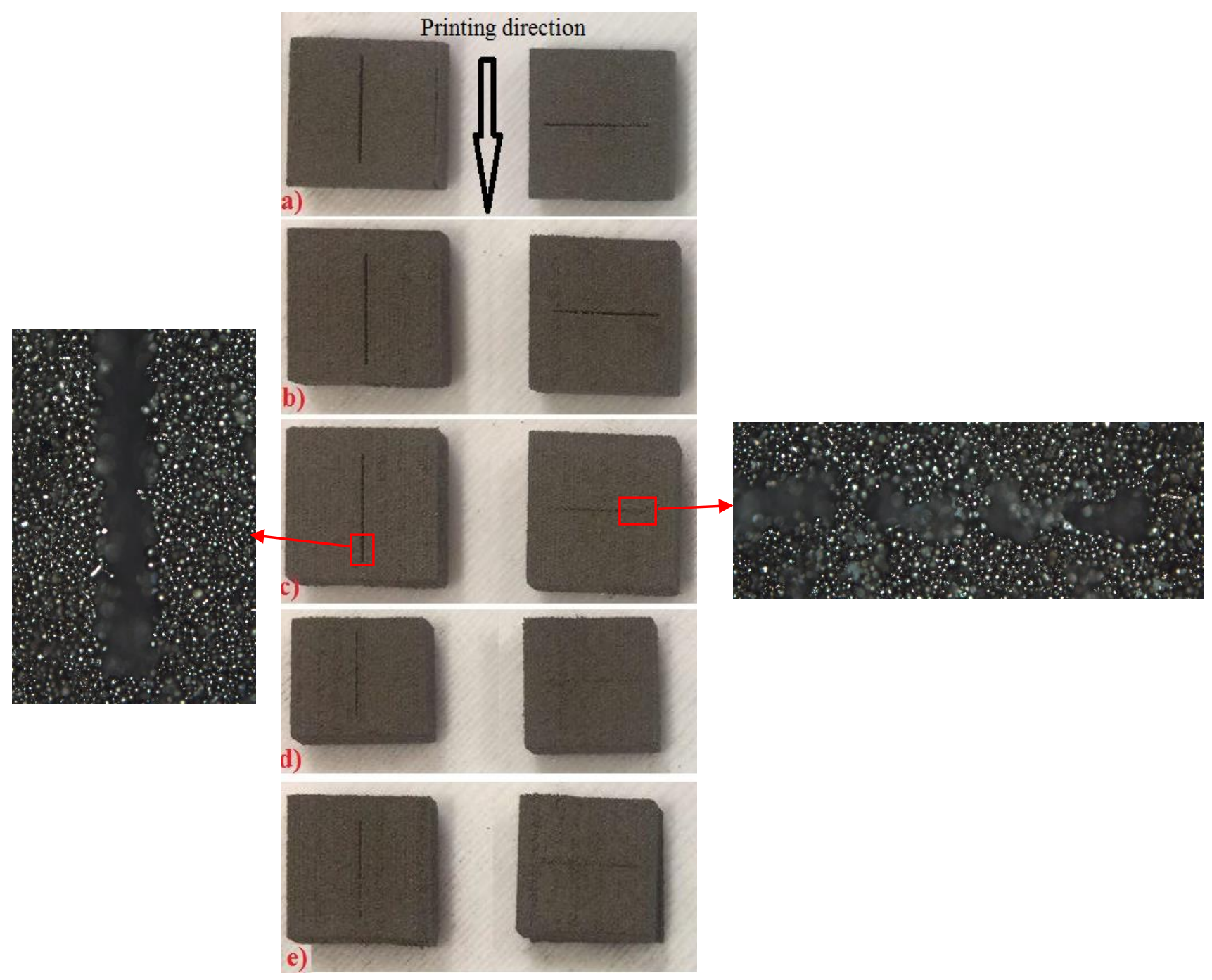

Fig. 5.4 Printed features with different printing speeds (a) $20 \mathrm{~mm} / \mathrm{sec}$ (b) 100 $\mathrm{mm} / \mathrm{sec}$ (c) $300 \mathrm{~mm} / \mathrm{sec}$ (d) $700 \mathrm{~mm} / \mathrm{sec}$ (e) $1000 \mathrm{~mm} / \mathrm{sec}$ 


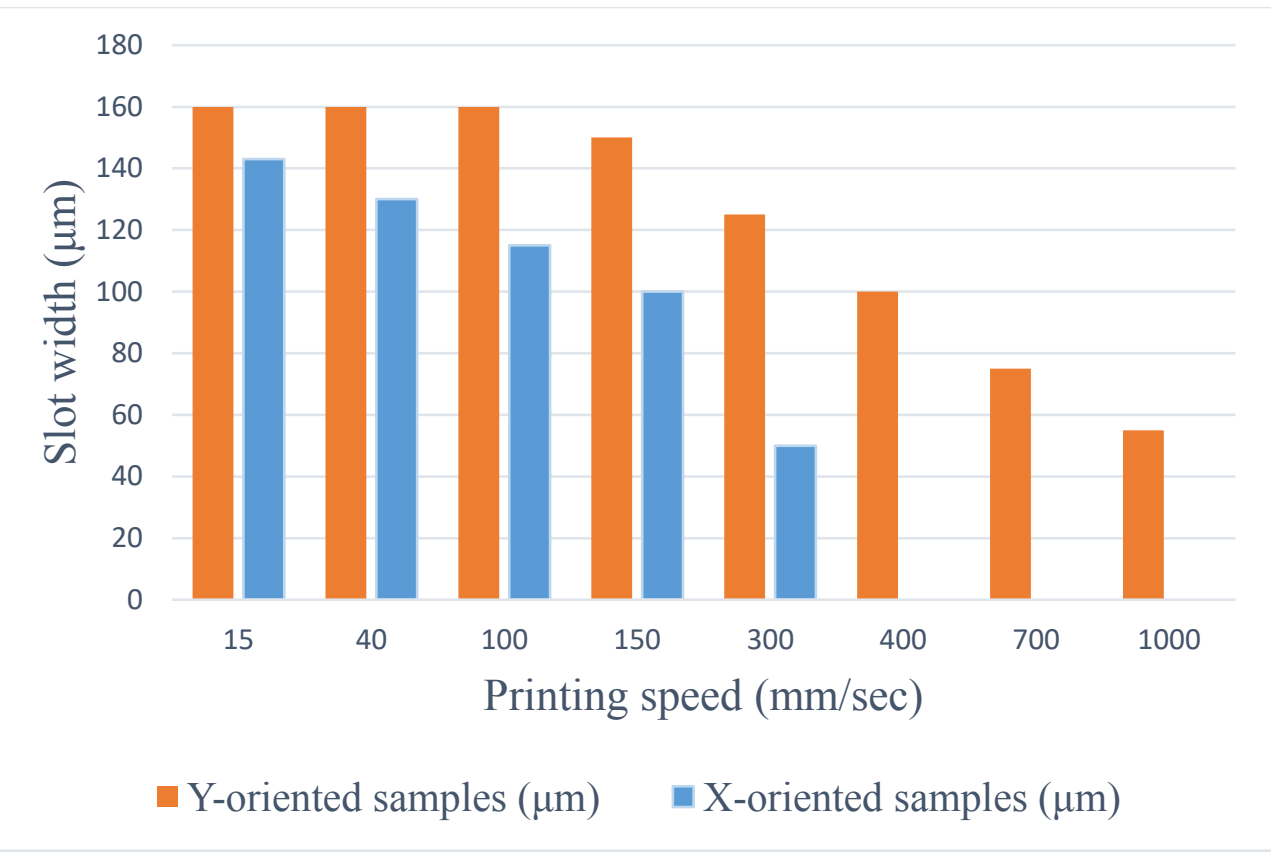

Fig. 5.5 Variation of the slot accuracy with printing speed and part orientation

Such dependency of the part dimensional accuracy on the printing speed can be attributed to velocity of the droplets jetted from the printhead. To analyze the behavior of an impinging droplet on the powder bed surface, the dimensionless Weber number which is commonly used in fluid mechanics for characterizing fluid interaction with a solid is implemented. The We number describes the ratio between the deforming inertial forces to stabilizing surface tension force and can be written as $[113,146]$ :

$$
\mathrm{We}=\rho \mathrm{r} v^{2} / \gamma
$$

where $\rho$ is the density of the binder material, $\mathrm{r}$ and $v$ are the radius and velocity of the droplet, respectively, and $\gamma$ the surface tension of the liquid binder. While at low Weber numbers the droplet behavior is dominated by the surface energy, which leads to weak deformation of the impinging droplet, a large value of this dimensionless number implies an inertia-dominated interaction between the impinging droplet and powder bed surface, which in turn affects the domain of spreading. In the literature, it has been frequently 
demonstrated that the velocity of a vertically impinging droplet on the surface of a porous medium is of significant effect on the maximum droplet spreading (wetted area) $[113,145$, 146]. The greater impact velocity, which is represented by higher Weber number, results in larger wetted area. Splashing might occur once the vertical velocity of the droplets exceeds a certain threshold, which is largely determined by the fluid properties, powder bed characteristics, and surface roughness [144]. For oblique impacts, droplet behavior becomes more complex due to the asymmetry of interaction and different impact patterns such as one-sided spreading or splashing might occur [142]. In BJ-AM process, impinging of the liquid binder droplets on the powder bed surface can be regarded as oblique impact particularly at high printing speeds. Therefore, overall velocity of a droplet released form the printhead can be theoretically resolved into its vertical and horizontal components as illustrated in Fig. 5.6. In the present study, the horizontal component of the drop velocity (referred to as printing speed) was altered whereas the vertical component (also called droplet release speed) was kept constant at nominal droplet release (exit) velocity, which is expected to be $8 \mathrm{~m} / \mathrm{s}$ based on the specifications of the system. Although the vertical velocity of droplets might slightly vary over $1.5 \mathrm{~mm}$ standoff distance between the printhead and the powder bed surface, such change in the droplet velocity is of negligible influence on the overall discussion of the current study. Therefore, it is assumed that the droplet release velocity remains unchanged before the impact. It is hypothesized that increasing printing speed most likely facilitates the droplet spreading along the printing direction ( $\mathrm{Y}$ direction) compared to the $\mathrm{X}$ direction due to the enhanced inertial forces in $\mathrm{Y}$ direction as shown schematically in Fig. 5.7. At very low printing speeds $(0.015-0.06$ $\mathrm{m} / \mathrm{s}$ ), as the droplet horizontal velocity is negligible compared to its vertical component (8 
$\mathrm{m} / \mathrm{s}$ ), the printed samples in both orientations are of approximately similar accuracies due to symmetrical spreading conditions. The effect of horizontal velocity of droplets within the intermediate range $(0.06-0.25 \mathrm{~m} / \mathrm{s})$ is enhanced along the printing direction, which leads to increased inaccuracy in X-oriented features (Fig. 5.4 and Fig. 5.5). For the case of high printing speed, splashing phenomenon is likely to occur during binder deposition through printhead due to the oblique impact of the droplets [142]. Therefore, at high printing speeds $(0.25-1 \mathrm{~m} / \mathrm{s})$ where the horizontal element of the velocity becomes more pronounced, apart from preferential spreading, splashing might also contribute to the loss of accuracy, which resulted in the loss of the slot features in the X-oriented printed parts and the reduced accuracy in the Y-oriented features.

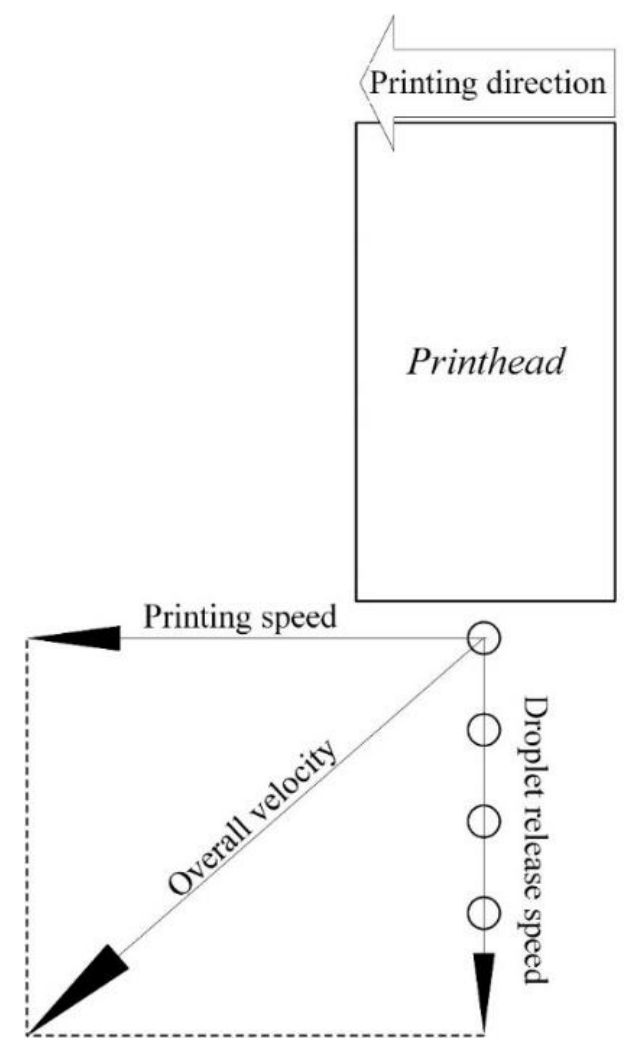

Fig. 5.6 Schematic graph of a droplet release from the printhead 


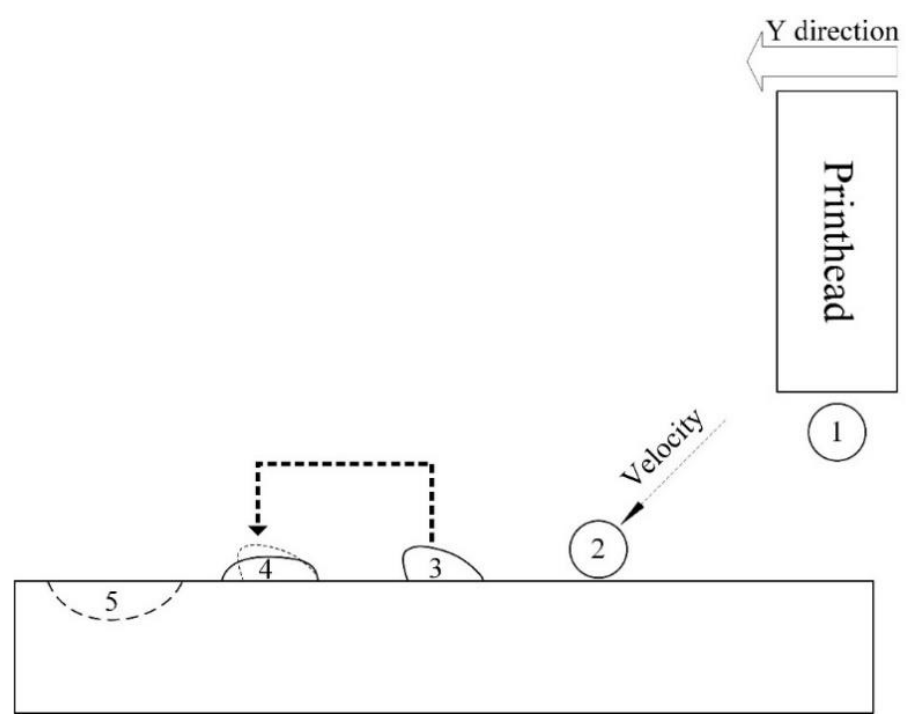

Fig. 5.7 Sequence of droplet impact phenomenon on the powder bed surface

There exists an abundance of literature about the effect of the material wettability on the fluid dynamics of an impinging droplet over a non-porous surface [147-151]. It has been shown that the tendency for droplet rebound off an impermeable surface increases when the static contact angle decreases (i.e. lower wettability) [141, 150, 152]. However, very limited studies have been devoted to investigating the effect of the material wettability on fluid dynamics of an impinging droplet on a porous surface. In [153], Rioboo et al. experimentally showed the effect of the wettability on rebounding of water droplets impacting on porous super-hydrophobic polymer surface. In BJ-AM, due to the high wettability of the used liquid binder with the examined powder material, i.e. low contact angle $\left(25^{\circ}\right)$ between the binder and 420 Stainless Steel, bouncing phenomenon in which the impinging droplet rebounds after the impact is less expected to contribute to the inaccuracy of the printed features. Therefore, droplet shifting from the point of impact along the printing direction due to bouncing seems unlikely to take place during the fabrication process. In order to investigate the occurrence of such shifting, different printing speeds were employed for fabrication of each individual cubic part as explained 
earlier in the methodology section. Fig. 5.8 illustrates the schematic representation of a sample printed with two different printing speeds and side view of the interface generated between the two sections. From the figure, it appears that the start location of the printed areas for both sections are aligned as indicated in Fig. 5.8, which would have been different if bouncing phenomenon was effective in printing process. On the other hand, the printed layers with printing speed of $300 \mathrm{~mm} / \mathrm{sec}$ exhibit a positive dimensional difference of 0.11 $\mathrm{mm}$ along the printing direction compared to the layers fabricated with $200 \mathrm{~mm} / \mathrm{sec}$ printing speed, indicating the influence of printing speed on droplet spreading dynamics after impact. This experiment verified the hypothesis that for the process parameters (e.g. droplet size, droplet release velocity) and the material system (liquid binder and powder) examined in the present research, the contribution of the droplet bouncing is negligible, and the primary source of error can be attributed to nonuniform droplet spreading due to increased printing speed.

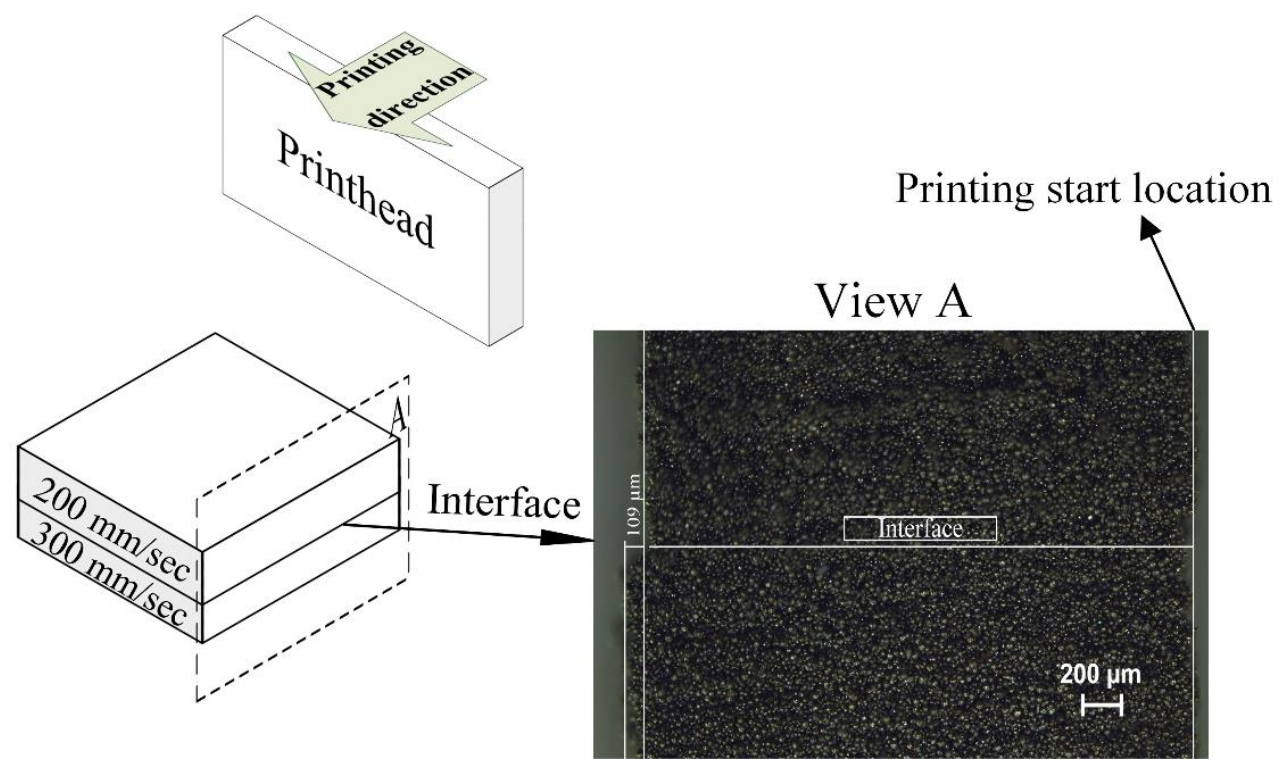

Fig. 5.8 Schematic representation of a sample printed with two different printing speeds 
The results of printing speed effect on dimensional accuracy of single-track, multitrack, and cubic features are shown in Fig. 5.9, Fig. 5.10, and Fig. 5.11, respectively.

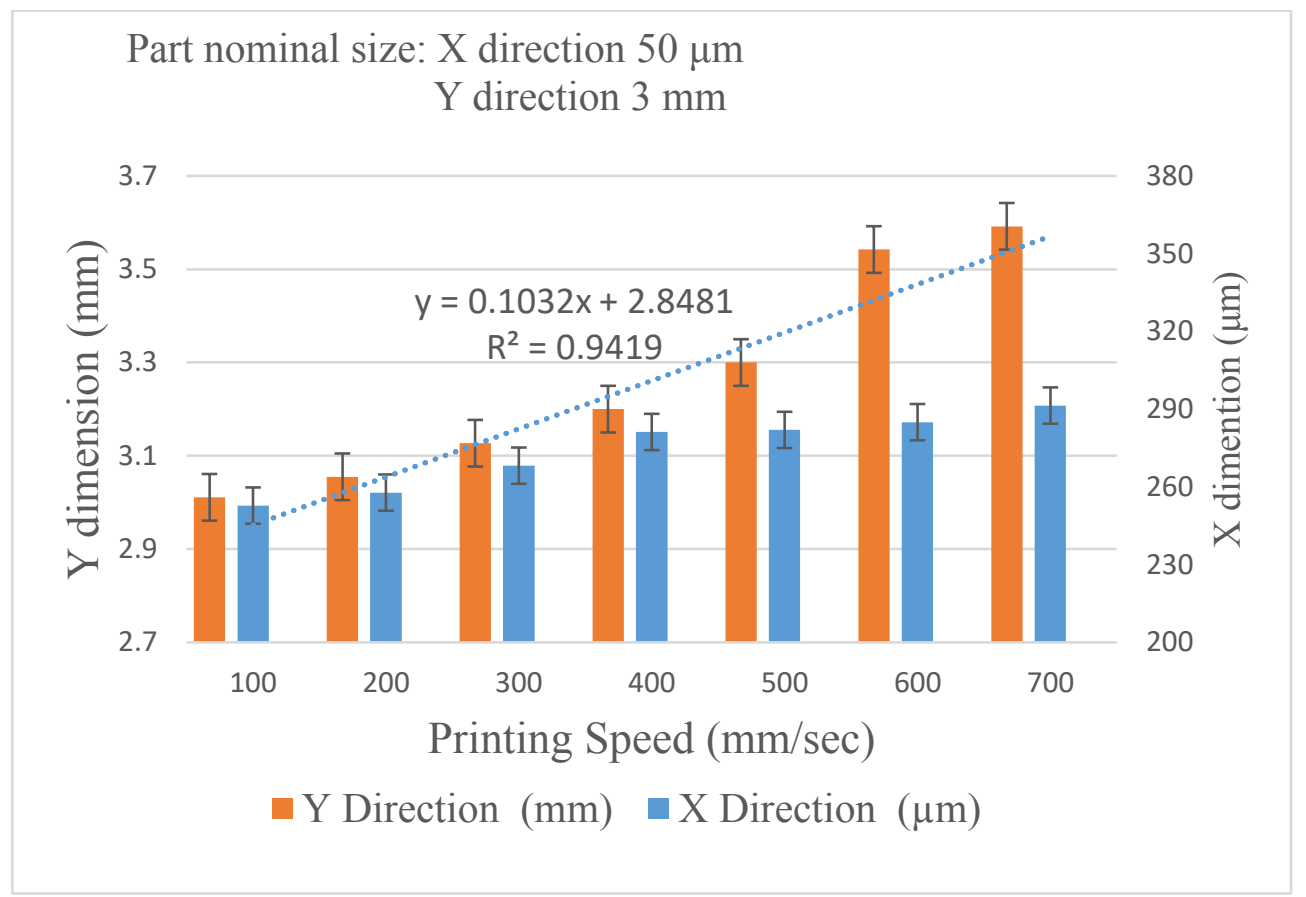

Fig. 5.9 Effect of printing speed on dimensional accuracy of single-track samples

Part nominal size: $\mathrm{X}$ direction $3 \mathrm{~mm}$

Y direction $3 \mathrm{~mm}$

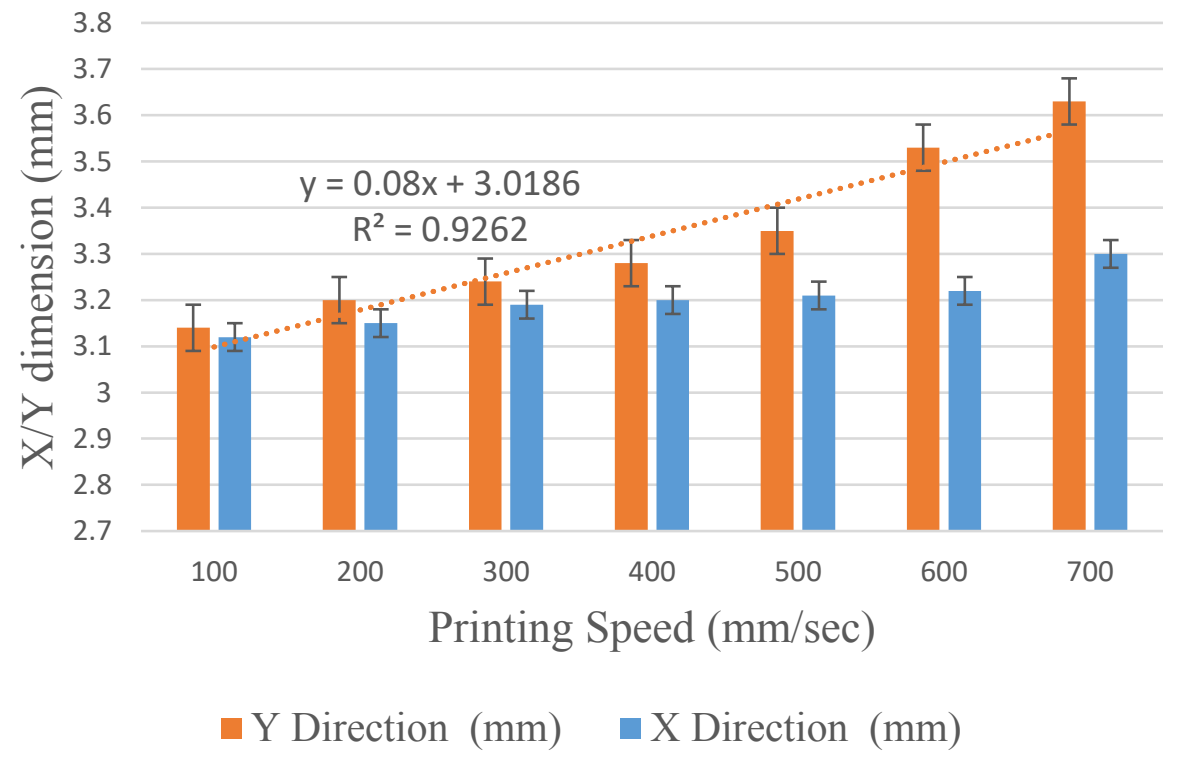

Fig. 5.10 Dimensional accuracy of multiple-track samples fabricated at various printing speeds 


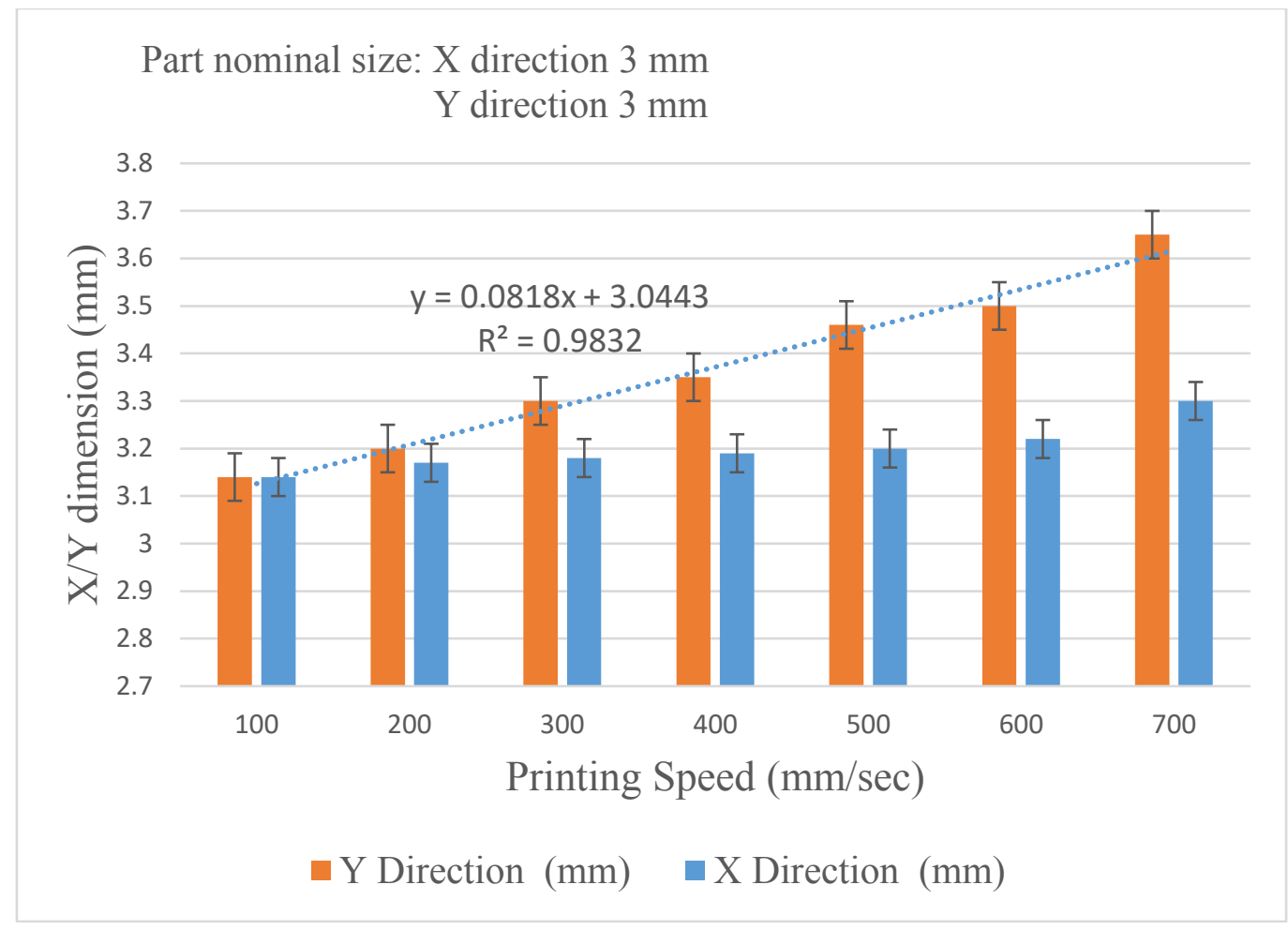

Fig. 5.11 Printing speed effect on accuracy of cubic parts

All the figures illustrate a general trend of increasing error with printing speed in both $\mathrm{Y}$ and $\mathrm{X}$ directions. Similar to the trend observed for the parts with open slots, these results also show that the effect of printing speed on accuracy in $\mathrm{Y}$ direction (printing direction) is greater than that of $\mathrm{X}$ direction (perpendicular to $\mathrm{Y}$ direction). From the figures, it is also noticeable that for the single-track samples the sensitivity of accuracy in $\mathrm{X}$ direction at different speeds is smaller compared to both multi-track and cubic samples over the range of print speed studied. For example, the maximum variation of accuracy for single-track features is $35 \mu \mathrm{m}$ in the $\mathrm{X}$ direction, while for multi-track and cubic samples this value increases to approximately $200 \mu \mathrm{m}$. Such behavior can be attributed to the raster scanning based droplet deposition pattern that is employed for part fabrication in the MLab printer. In the raster scanning, each nozzle of the printhead is utilized for successive droplet depositions in the $\mathrm{Y}$ direction followed by the powder bed movement in the $\mathrm{X}$ 
direction (Fig. 5.2) to cover the entire designated area. Overlapping of droplets deposited in the $\mathrm{Y}$ direction by each nozzle forms individual lines (referred to track in this study). With this binder delivery pattern, fabrication of a $2 \mathrm{D}$ feature within each layer is accomplished by ensuring the binding strength between such tracks. It is worth mentioning that the printhead jets the binder droplets only in $\mathrm{Y}+$ direction (i.e. jetting is not performed when the printhead moves in Y- direction). As illustrated in Fig. 5.12, for multi-track and cubic samples, if the spacing between successive tracks is such that considerable overlaps between tracks occur, the "expansion" of deposited track dimensions could be greatly exacerbated by liquid binder supply from the adjacent tracks, which leads to further increase of dimensional inaccuracy. It is worth mentioning that during the printing the degree of droplet overlapping was determined by the process software and depended on other process settings such as saturation level and layer thickness. As all the builds in the present study were fabricated using droplets with constant volume, higher saturation level and larger layer thickness would result in more excess droplet supply in printed areas, which would consequently lead to more significant droplet spreading and decrease of absolute dimensional accuracy. 


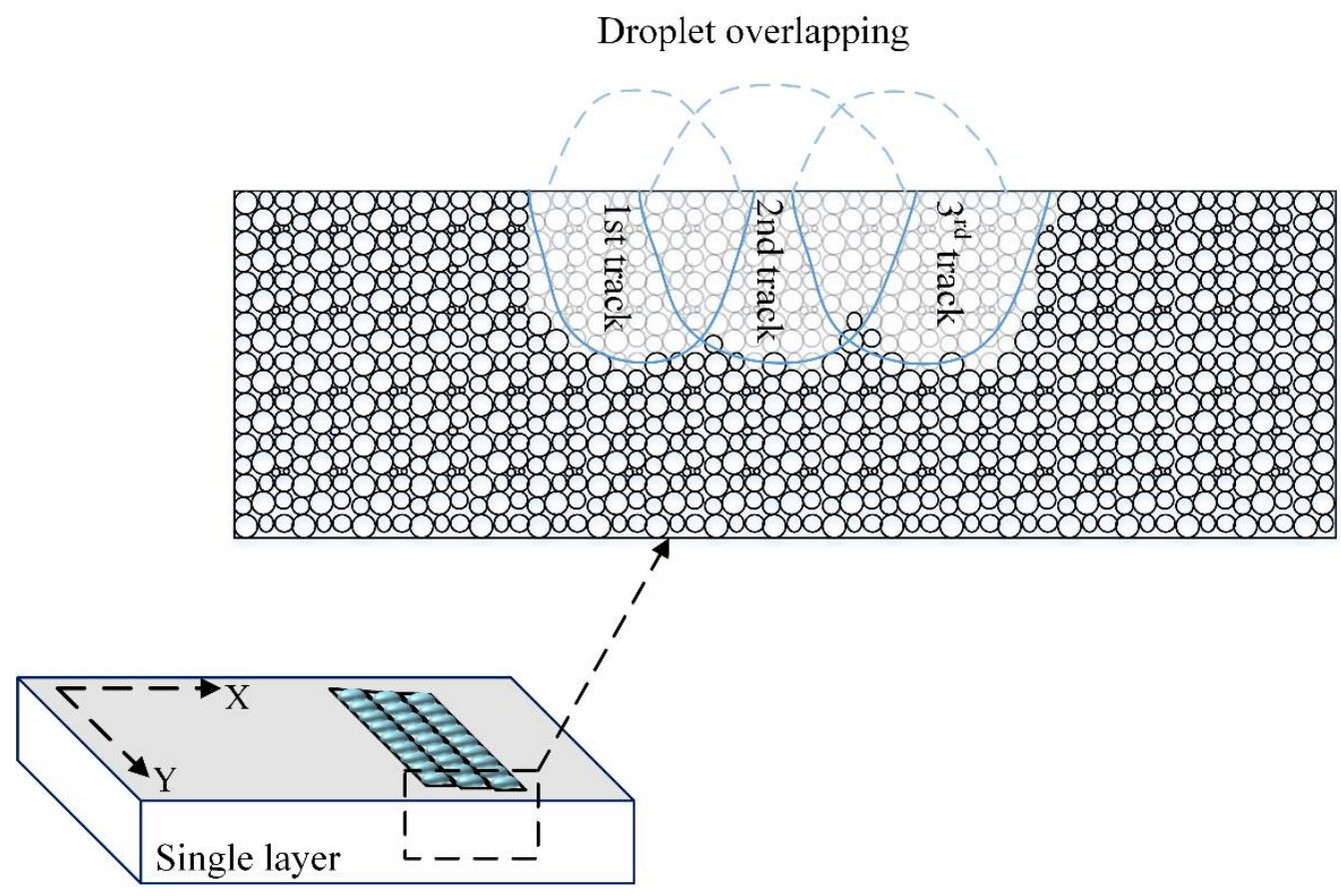

Fig. 5.12 Schematic of feature formation inside a layer

To describe the quantitate correlation between the printing speed and the dimensional accuracy of the samples in Y direction, which is significantly influenced by printing speed, linear models were fitted to the empirical data as shown in Fig. 5.9, Fig. 5.10, and Fig. 5.11. The high R-squared values of these linear models indicate the linear equations fitted to the data can be reliably used to predict the variation of the dimensional accuracy of the features with printing speed for the set of the liquid binder and the powder material used in current study. It should be noted that these equations are highly dependent on the powder feedstock characteristics and the liquid binder properties, since they largely determine the interaction between liquid binder and powder upon impact and permeation $[144,154]$.

The effect of printing speed on the variation of equilibrium saturation was determined experimentally from cubic samples with printing speed ranging from $100 \mathrm{~mm} / \mathrm{s}$ 
to $700 \mathrm{~mm} / \mathrm{s}$ as shown in Fig. 5.13. It appears that a linear correlation could be established for the correlation between the saturation level of fabricated parts and the printing speed. Such a linear trend might be mainly attributed to the decrease of dimensional accuracy with printing speed, which is manifested in increase of dimensions of printed samples. In other words, higher printing speed results in the increased volume of the printed samples. As the nominal binder amount used for fabrication remains constant, the increased volume leads to lower equilibrium saturation. Decreasing the equilibrium saturation levels would deteriorate the mechanical strength of the printed green parts [13, 14]. Therefore, applying higher printing speeds for part fabrication in BJ-AM process would influence not only the dimensional accuracy but also the strength of the printed components. Therefore, during the fabrication of complex geometries, it is preferable to use lower printing speeds if high resolution is required.

In addition to the printing speed, other parameters such as powder material physical properties (e.g. densities, wettability) and liquid binder characteristics (e.g. viscosity, surface tension) could also potentially influence the quality of the fabricated parts in BJAM process. As the binder permeation process in the BJ-AM process is closely associated with the binder and powder bed characteristics, additional works are needed to establish more comprehensive knowledge about the potential impacts of these factors as well as their interactions. 


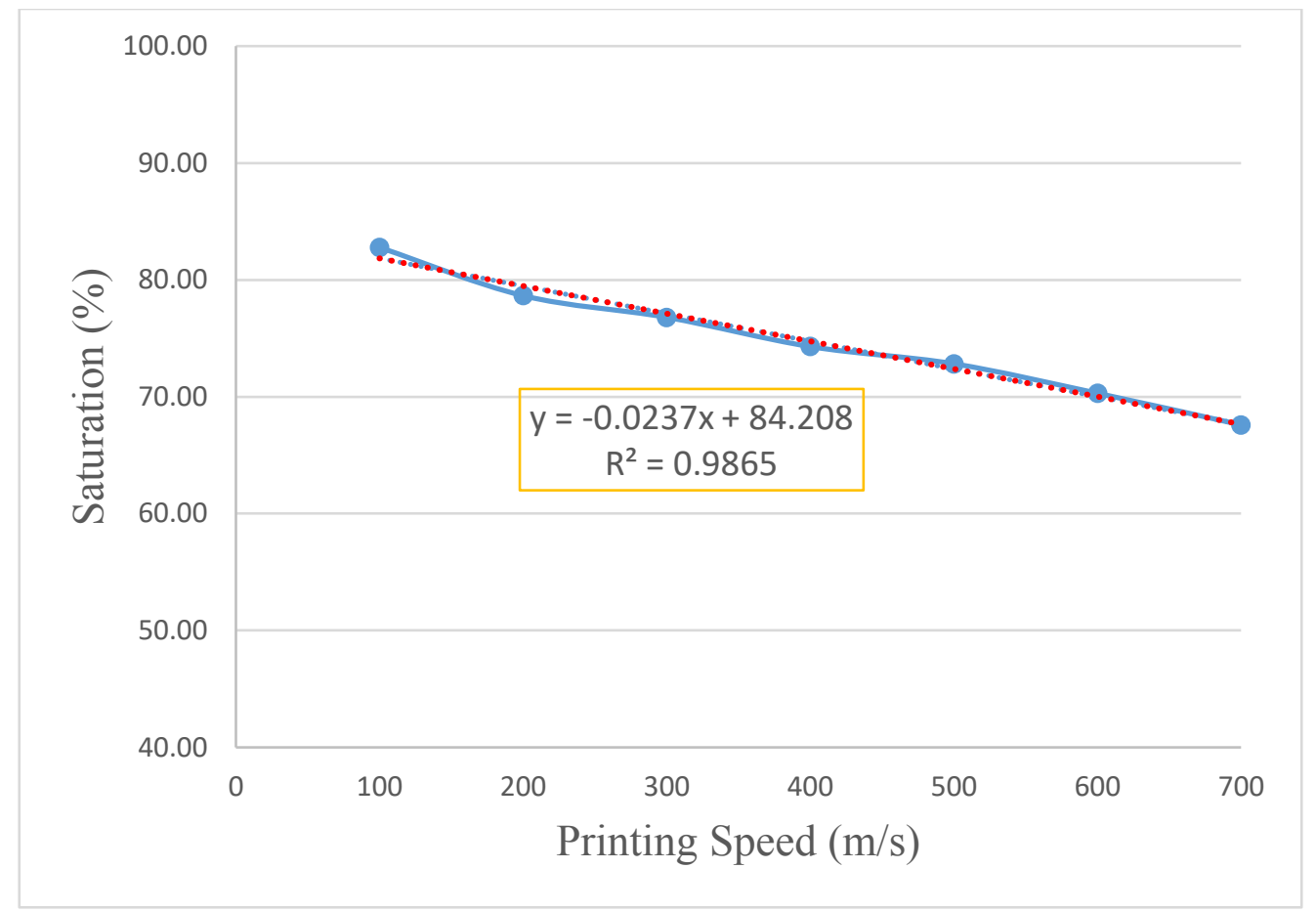

Fig. 5.13 Saturation variation with printing speed for cubic samples

\subsection{Conclusion}

In the present paper, the effect of the printing speed on quality and integrity of the printed components is experimentally evaluated. It is shown that increasing printing speed reduces the accuracy of the fabricated pars regardless of their orientation in the powder bed due to the enhanced inertia forces. Also, it is observed that the accuracy of parts printed in X-direction differs with that of Y-oriented samples which might be partly contributed by the asymmetrical spreading of the droplets favored toward the printing direction, which arises mainly from the horizontal velocity of droplets. In addition, experiments demonstrate that the droplet shifting from the impact point due to the droplet bouncing does not seem to contribute the inaccuracy of the printed features. The experiments suggested linear correlation between the printing speed and dimensional accuracy of printed features in $\mathrm{Y}$ direction (printing direction). It was also observed that the saturation level of printed 
samples is affected by printing speed due to dimensional change of printed samples. Further investigation coupled with high speed video that can capture the moment of droplet impact on the powder bed surface could potentially provide more insight into the effect of printing speed on the droplet impact and consequently the quality of the fabricated components. 


\section{CHAPTER 6}

\section{EFFECT OF POWDER MATERIAL CHARACTERISTICS ON QUALITY OF PARTS FABRICATED VIA BINDER JETTING PROCESS}

\subsection{Introduction}

In the binder jetting additive manufacturing (BJ-AM) process, drops of a binding liquid agent are deposited on the powder bed to create bonds between particles in the selective areas. After the binder deposition, the whole surface area of the printed layer including the saturated and unsaturated regions is exposed to external heating with a predetermined intensity, usually via a radiation heating source (e.g. infrared lamp). This external heating serves to partially cure the binder and ensure adequate mechanical strength for consequent printing steps. The process of powder spreading, binder deposition and inprocess curing of the binder are repeated for each layer until the entire geometry is fabricated as schematically illustrated in Fig. 6.1. The fabricated green parts in this stage are further heated normally at $200{ }^{\circ} \mathrm{C}$ for approximately 2 hours to enhance the mechanical strength and ensure the structural integrity of the components during handling and excess powder removal process. The green parts fabricated by the BJ-AM printing process possess only limited strength and often requires additional post-processing such as sintering and infiltration in order to achieve desired mechanical performance $[12,155,156]$. The post- 
printing processes generally involve the debinding step aimed to remove the binder material followed by a densification step that reduces or eliminates internal voids and enhances mechanical properties of the final structures. Therefore, it can be concluded that the quality of the final parts produced by BJ-AM technology is determined not only by the parameters related to green part fabrication process but also by the post-printing processes and affecting factors [14].

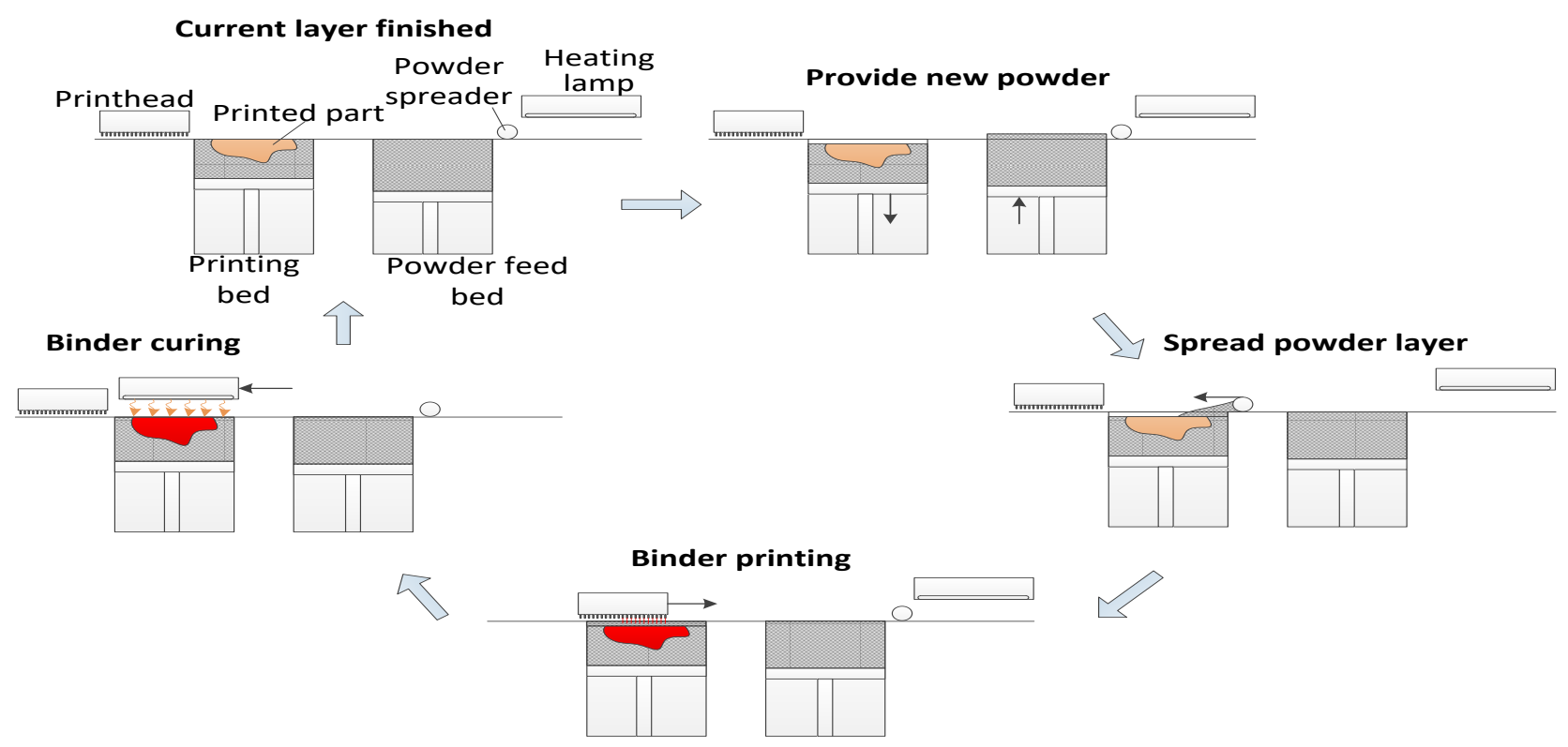

Fig. 6.1 Schematics of BJ-AM process steps

There exist multiple factors that could potentially influence the feature resolution and quality of the green components fabricated via BJ-AM process such as binder and powder physical properties, powder bed characteristics, droplet impact velocity, and powder material wettability to the liquid binder $[13,95,155,157,158]$. One of the key parameters in determining the minimum feature size and final part quality in BJ-AM process is the interactions between the liquid binder droplets and the powder materials. While the lateral spreading of the impinging droplets might negatively impact the 
dimensional accuracy of the printed geometries, controlled spreading of the binder drops after impact leads to enhanced dimensional accuracy and improved attainable minimum feature size, which might be achieved by fast vertical penetration of the binder liquid into the powder bed. After binder deposition, the flow dynamics of the impinging droplets on the target surface including vertical penetration and lateral spreading would be influenced by many factors including powder bed characteristics $[95,158,159]$, binder and powder material physical properties, droplet impact velocity, and powder wettability to the binder $[86,160,161]$. Many of the powder bed physical characteristics such as pore size, packing density, flowability and surface area are primarily determined by the powder particle morphology including the particle size distribution and the shape of the particles $[46,95$, 162]. It has been shown by different researchers that smaller powder particle size facilitates the expansion of the droplets due to smaller pore sizes, which would consequently reduce the accuracy of the fabricated structures $[86,95]$. On the other hand, powder beds with larger particles are expected to facilitate the binder vertical penetration due to the increased powder bed permeability with larger pores. Therefore, it is clear that powder material characteristics would affect the binder-powder bed interaction and consequently the dimensional accuracy of the printed components.

The effect of particle size distribution on the surface finish of additively manufactured parts has been demonstrated by different researchers in the literature $[157$, 163]. Generally, smaller particles result in improved surface quality of components due to the diminished staircase effect, as well as the use of thinner layers $[157,164]$. One the other hand, although the influences of particle size distribution on different aspects of the AM process characteristics such as powder bed packing density, flowability, part surface 
texture, and mechanical properties have been explored in various powder bed based processes [163-165], there exists very limited study on effect of particle size distribution on the quality of green/post-processed structures manufactured by the BJ-AM process. Therefore, in the present work the qualitative/quantitative correlations between the powder particle characteristics and the quality of parts including surface finish, dimensional accuracy as well as mechanical strength were experimentally evaluated.

\subsection{Methodology}

In the current study, three different types of the 316L stainless steel powder (LPW Technology) with different mean particle size and particle size distribution were used for the experiments. The characteristics of the LPW SS 316L powder types used for the experimentations are listed in Table 6.1.

Table 6.1 316L stainless steel powders used for the experiments

\begin{tabular}{|c|c|c|c|}
\hline Powder types & D10 & D50 & D90 \\
\hline Type I & $8 \mu \mathrm{m}$ & $14 \mu \mathrm{m}$ & $20 \mu \mathrm{m}$ \\
\hline Type II & $20 \mu \mathrm{m}$ & $31 \mu \mathrm{m}$ & $44 \mu \mathrm{m}$ \\
\hline Type III & $54 \mu \mathrm{m}$ & $78 \mu \mathrm{m}$ & $105 \mu \mathrm{m}$ \\
\hline
\end{tabular}

The morphology of the powders including particle shape and size distribution of each type of powder is also shown in Fig. 6.2. The particle size distribution analysis was conducted using Microtrac S3000 and scanning electron microscope (SEM) micrographs of the powders were taken by and Zeiss Supra 35 microscope. From the results, all three powder types are comprised of spherical particles with unimodal size distribution of 14 $\mu \mathrm{m}, 31 \mu \mathrm{m}$ and $78 \mu \mathrm{m}$ mean particle diameters for powder types I, II and III, respectively. 
In order to assess the influence of the powder material characteristics on the interaction between the binder and powder beds, a real time imaging system using Fastec Imaging HiSpec 2 hig hspeed camera was developed. As shown in Fig. 6.3, the system consists of a micropipette (0.5-10 microliter droplet volume size), the high speed camera, mechanical fixtures for holding the micropipette and the camera, and a light source $(150 \mathrm{~W}$ Fiber Optic Dual Gooseneck Microscope Illuminator) . Individual droplets of $2 \mu$ volume were deposited using the setup, and real-time flow dynamics of the impacting droplets on different powder beds were recorded. The diameter and depth of the resulting liquidpermeated features were measured by an optical microscope (Olympus MX51 with Olympus QColor 5 digital camera). Afterwards, batches of cubic samples with $8 * 8 * 8 \mathrm{~mm}^{3}$ dimensions were printed using the three different powder types so as to investigate the qualities of the three-dimensional (3D) structures printed by BJ-AM process using these types of material feedstock. 

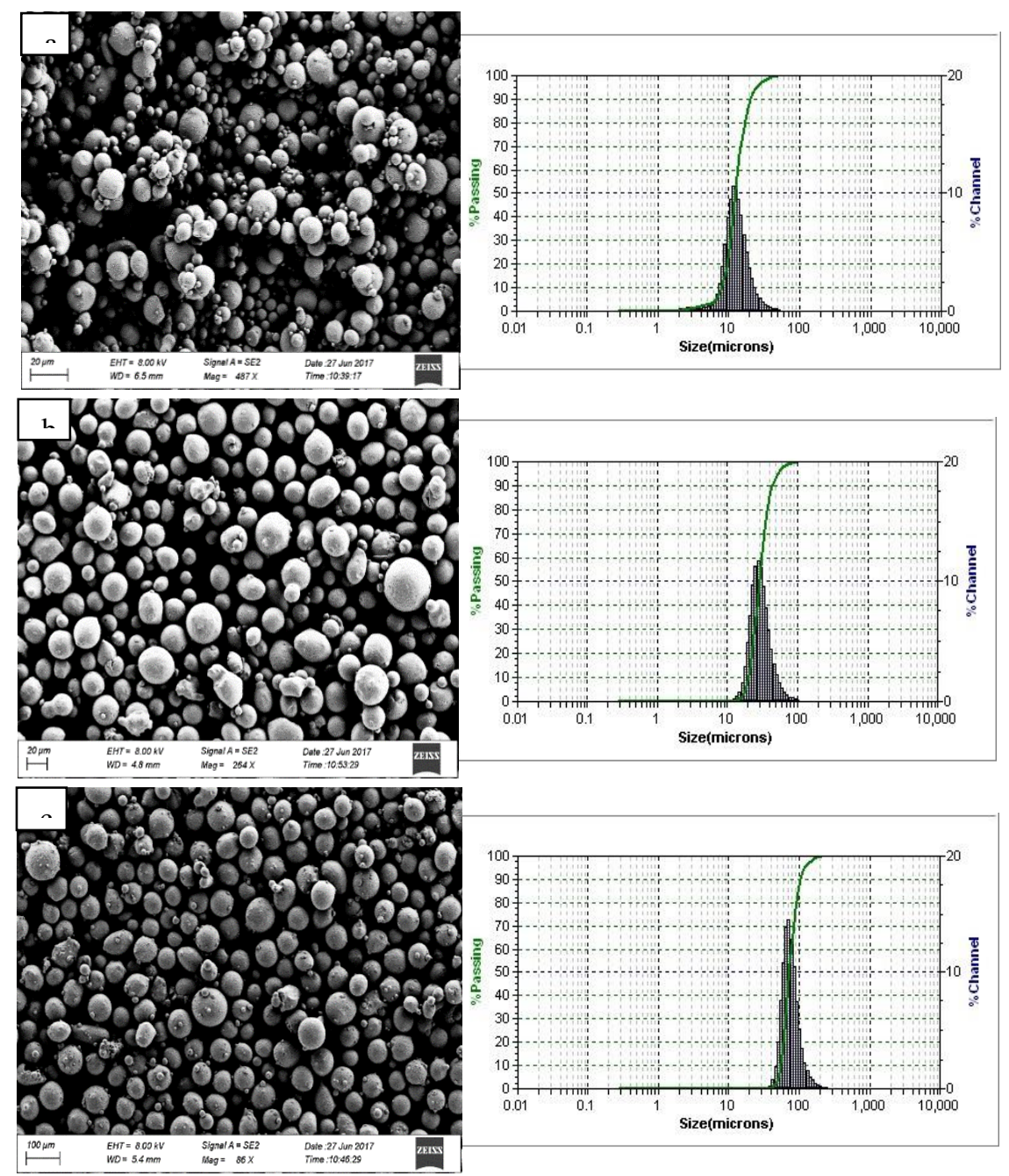

Fig. 6.2 SEM images of 316L SS powder with different size distributions a) powder type I with 5-25 size distribution b) powder type II with 15-45 size distribution c) powder type III with 44-106 size distribution

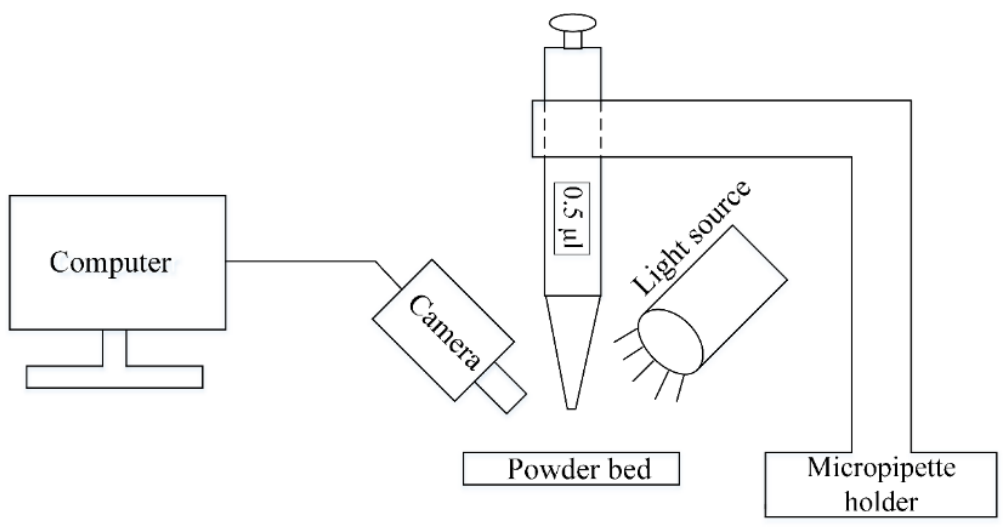

Fig. 6.3 The schematic of high-speed imaging setup 
The BJ-AM system used for the binder/powder material interaction experiments and sample fabrication was the ExOne M-Lab printer. The binder used for the fabrication of all the samples was the ExOne PM-B-SR1-04, which is an ether solvent-based binder compatible with the M-Lab system. To evaluate the effect of the particle size distribution on the packing density of the powder beds, rectangular cups with the inner dimensions of $30 * 50 * 20 \mathrm{~mm}$ were printed and retrieved after binder curing at $200{ }^{\circ} \mathrm{C}$ for at least 2 hours to determine the mass of the layered powder and volume occupied inside the cup. Given the bulk density of the material, powder weight and the inner volume of the cup, the packing density of each powder type was calculated. For measuring the dimensional characteristics of the samples, digital caliper, micrometer, and/or the optical microscope were utilized depending on the size and structural integrity of the parts examined.

For all the parts fabricated via M-Lab printer, the process parameters were set up according to Table 6.2. Additional information about the interpretation of each process parameter can be found elsewhere $[14,55,166]$. Also, the powder bed packing density values of $51 \%, 57 \%, 61 \%$ for powder types I, II, and III respectively were used for the printing saturation level setup according to the measurement results. For calculation of the saturation level, the powder bed pore volume is needed which can be determined from the powder bed packing density. It is worth mentioning that the saturation is described as the amount of pore volume filled with a liquid binder in a predefined envelope [14]. 


\section{Table 6.2 Printing parameters used for the M-Lab BJ-AM system}

\begin{tabular}{|c|c|}
\hline Process parameter & Value \\
\hline Powder spreading speed (mm/s) & 2 \\
\hline Saturation level (\%) & 100 \\
\hline Drying power (\%) & 80 \\
\hline Drying time (s) & 45 \\
\hline Printing speed (mm/s) & 150 \\
\hline Layer thickness (mm) & 0.15 \\
\hline
\end{tabular}

The surface roughness of the fabricated samples was analyzed using Veeco Dektak

$8 \mathrm{M}$ Profilometer to determine the correlations between the surface roughness of the samples before/after post-printing processes and the powder characteristics. The results were reported as the average of five replicate measurements per sample.

To investigate the effect of the particle size on the density of the fabricated structures after the sintering, 8 cubic samples from each powder type were fabricated and sintered in argon atmosphere. The sintering process included the debinding at $500^{\circ} \mathrm{C}$ for one hour with a heating rate of $20^{\circ} \mathrm{C} \mathrm{min}{ }^{-1}$, followed by the sintering at $1300{ }^{\circ} \mathrm{C}$ for 90 minutes with $10{ }^{\circ} \mathrm{C} \mathrm{min}^{-1}$ heating rate, then furnace cooling to the room temperature. The densities of the sintered specimens were measured using Archimedes' method, and the microstructure were analyzed using the SEM. For the SEM characterization, the sintered samples were cold mounted with epoxy resin, then ground flat using grit papers from 180 
to 1200 grit, and finally polished with 9, 3,1-micron diamond paste in sequence of increased fineness. The samples prepared for SEM characterization were also used to measure the microhardness using a Shimadzu HMV-G microhardness tester. The Vickers indentation testing was carried out at random spots on the sample surface, and at least 10 measurements were taken for each sample.

\subsection{Result and discussion}

\subsubsection{Powder packing density}

The packing density of the powder beds is one of the key factors that could significantly affect the quality and integrity of the components in BJ-AM technology. The powder packing density determines the pore volume inside the powder bed which can be occupied with the liquid binder, which in turn influences the green part mechanical strength. Inaccurate determination of packing density of a powder material would result in either excessive binder deposition or insufficient binder supply that would in turn compromise the part quality. Furthermore, both the final density and the sintering shrinkage of the fabricated components are also influenced by the powder bed packing density [157, 167]. Therefore, insights into the quantitative/qualitative characteristics of the packing density are of crucial importance for the understanding of the part quality. It is well know that the packing density of a powder bed is dependent upon particle size distribution, particle shape, and the powder spreading system [168, 169]. It was shown that the effect of the mean particle size on the packing density is less significant $[46,170]$, whereas broadening particle size distribution appears to favor higher packing densities due to the void-filling effect of fine particles $[46,171-174]$. For the case of spherical particles 
used in the present study and the counter-rotating roller mechanism used for powder bed formation, similar dependency of packing density to particle size distribution was observed as shown in Fig. 6.4. As the size distribution of the particles increases, the powder bed packing density increases. Powder type I, which has the narrowest size distribution, results in a powder bed packing density of 51\%, whereas powder type III with wider particle size distribution exhibits higher packing density of $61 \%$.

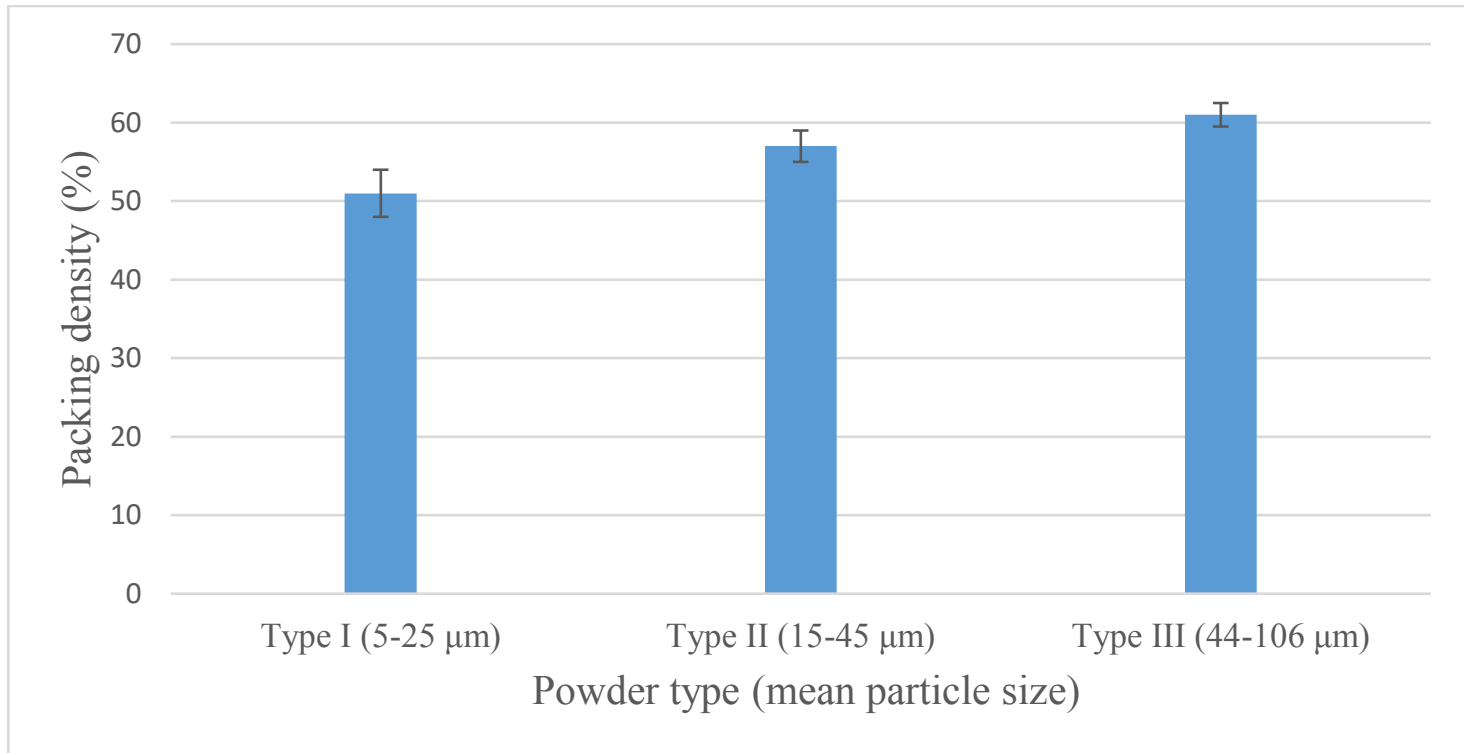

Fig. 6.4 Packing density of the different powder types with the polydispersity index

\subsubsection{Powder/binder interaction and accuracy}

Fig. 6.5 shows the powder cakes from the interactions between single binder droplets and the powders with different types. The structures exhibit semi-spherical geometries with varying values of the diameter and height, which correspond to the spreading diameter and depth of penetration of the droplet in the powder beds, respectively. 


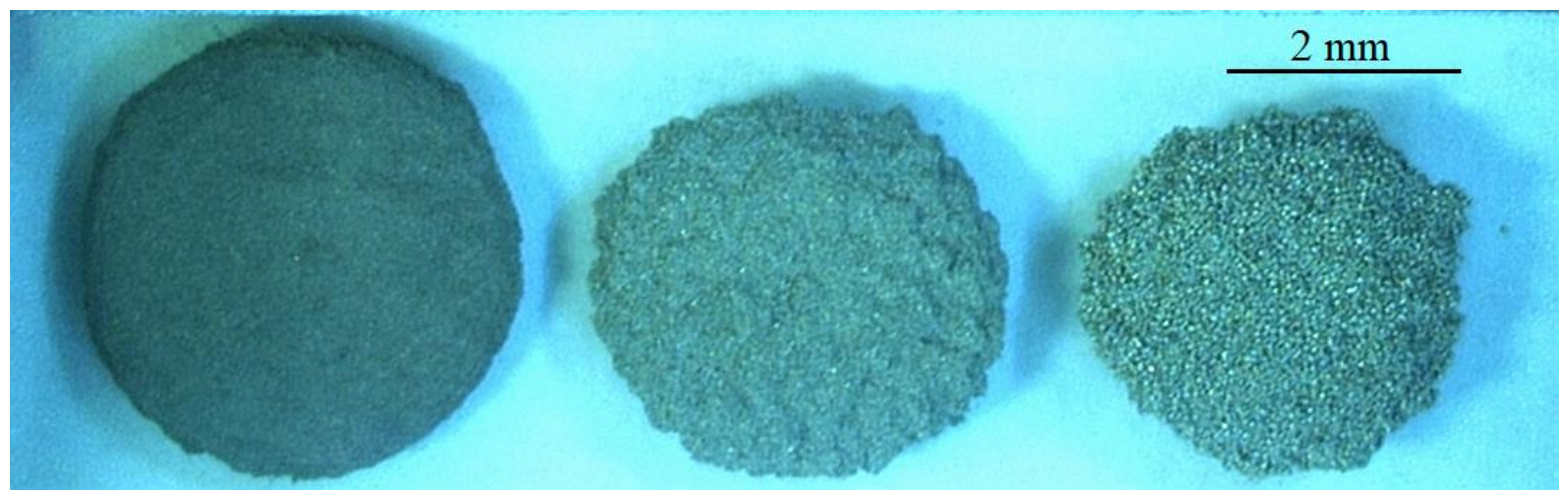

Fig. 6.5 The formed features resulted from the interaction of a single binder droplet

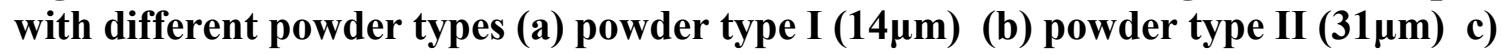
powder type III $(78 \mu \mathrm{m})$

The quantitative results of the single droplet binder/powder bed interaction are shown in Fig. 6.6. From the results, the features formed by the powder type III exhibit the highest depth and lowest diameter. While the spreading diameter of the droplets in the powder bed decreases with increasing mean particle size, the final depth of the droplet penetration exhibits the opposite trend. Such characteristics of binder/powder material interaction can be attributed to the change of the macroscopic surface roughness and pore morphologies. The surface roughness of the powder beds is primarily determined by the powder characteristics such as particle shape and particle size distribution as well as the powder spreading quality. For powder with spherical particle morphologies, the larger mean particles size would generally result in powder beds with rougher surfaces. The surface roughness would in turn influence the fluid dynamics of the droplet impinging on the powder bed surface in the droplet impact-driven spreading phase. In this phase, the droplet spreading is mainly driven by the droplet kinetic energy from the impact. The surfaces with greater roughness would impede the droplet impact-driven spreading over the surface $[112,175,176]$. As the drop spreading speed upon the impact decreases for powder beds with rougher surfaces, the vertical penetration of the droplet into the powder 
materials is effectively enhanced by increased liquid supply which would otherwise be more rapidly depleted from the surface due to the spreading. Fig. 6.7 shows the real-time images of the interaction between a binder droplet and different powder beds at the end of impact-driven spreading phase. These images clearly show that the powder bed formed with smaller particles (Fig. 6.7 a) would result in larger spreading diameter at the end of impact-driven spreading phase as previously explained.



Fig. 6.6 Depth and diameter of single drop features vs mean particle size of powdered materials

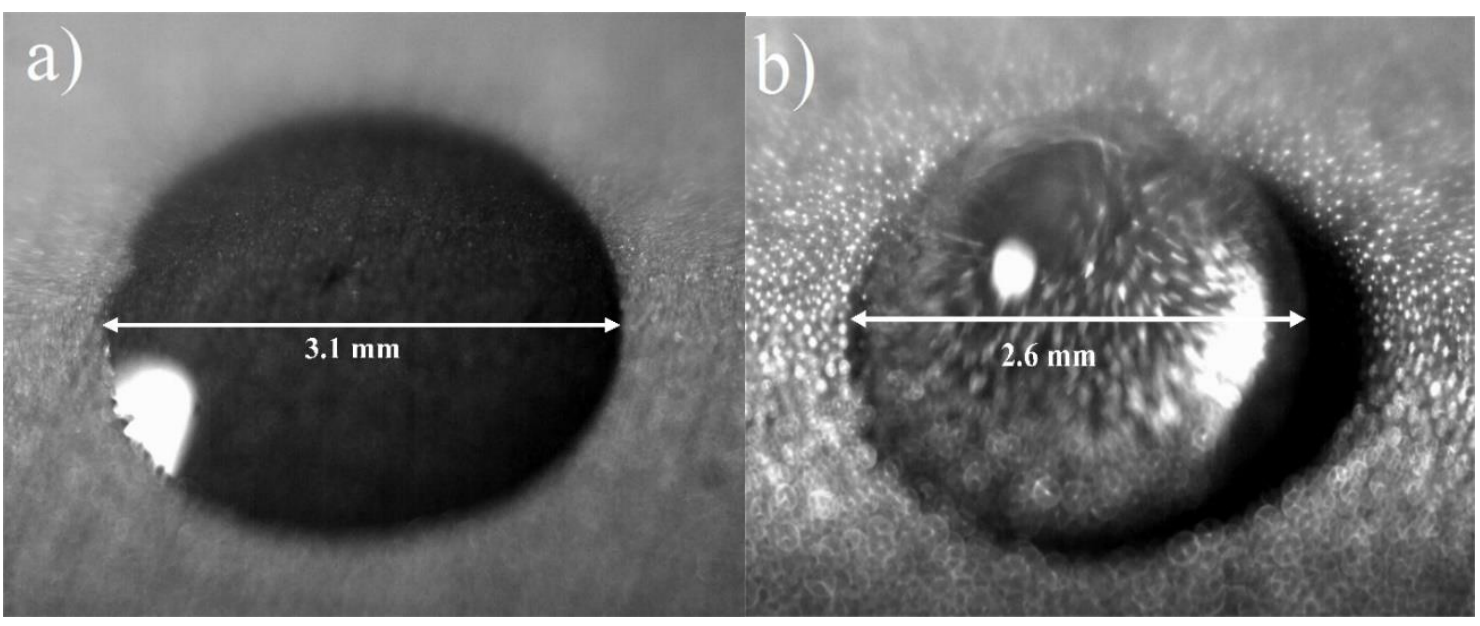

Fig. 6.7 Interaction of a binder droplet with different powder types at the end of impact-driven phase a) powder type I b) powder type III 
After the impact-driven spreading phase of the droplet ends, the capillary-driven spreading phase becomes the dominant mechanism $[91,113,158]$. In this stage, the powder beds with larger pore sizes facilitate the binder permeation into the porous structure due the improved permeability [95], which can be quantified by the Kozeny equation that correlates the permeability with the mean diameter of particles $[177,178]$ :

$$
k=a \frac{\epsilon^{3} D^{2}}{(1-\epsilon)^{2}}
$$

Where $\epsilon$ is the porosity of the powder bed, D is the mean diameter of powder particles, $\mathrm{k}$ is the permeability of the powder bed, and a is a constant. The volume of a droplet penetrated a powder bed at any given time, $V_{p}(t)$, can be estimated from the following equation [53]:

$$
V_{p}(t)=\frac{K}{2} \int_{0}^{t} \frac{r^{2}(t)}{\sqrt{t}} d t
$$

Where

$$
K=8 \pi k \sqrt{\frac{\gamma \cdot \cos \theta}{2 \eta \cdot R_{\text {pore }}^{3}}}
$$

Where $r(t)$ is the instantaneous radius of the spreading droplet at time $t, R_{\text {pore }}$ is the pore radius (mean pore radius from the BET measurements), $\gamma$ is the surface tension of the liquid, $\theta$ is contact angle of the binder droplet on the material, and $\eta$ is the droplet liquid viscosity. 
From Eq. (6.2), the higher permeability (k) would lead to the rapid droplet penetration into the powder bed. Therefore, for the powder beds with large particles, the droplet spreading over the surface might be reduced by the rapid depletion of binder droplet from the surface due to enhanced penetration. Therefore, for both stages of the dropletpowder bed interactions, the powder beds with larger particle sizes would facilitate the droplet vertical penetration and impede the binder droplet spreading.

Such droplets-powder bed interaction characteristics were also observed in cubic specimens $\left(8 \times 8 \times 8 \mathrm{~mm}^{3}\right)$ as shown in Fig. 6.8. Consistent with the results obtained from the single-droplet experiments, the samples fabricated from powder with smaller particle sizes exhibit higher inaccuracies in the lateral directions ( $\mathrm{X}$ and $\mathrm{Y}$ directions) compared to those printed from powder with larger particle sizes. This contrasts with the previous perception that smaller powder particle size would always be beneficial to the accuracy of the printed structures. On the other hand, the accuracy of samples in the $\mathrm{z}$ direction (binder vertical penetration) decreases with the increasing mean particle size. Considering that the layer thickness used for this study is $150 \mu \mathrm{m}$, the large particle size of the Type III powder likely contributed to the drastic reduction of the z-accuracy due to insufficient powder spreading. 


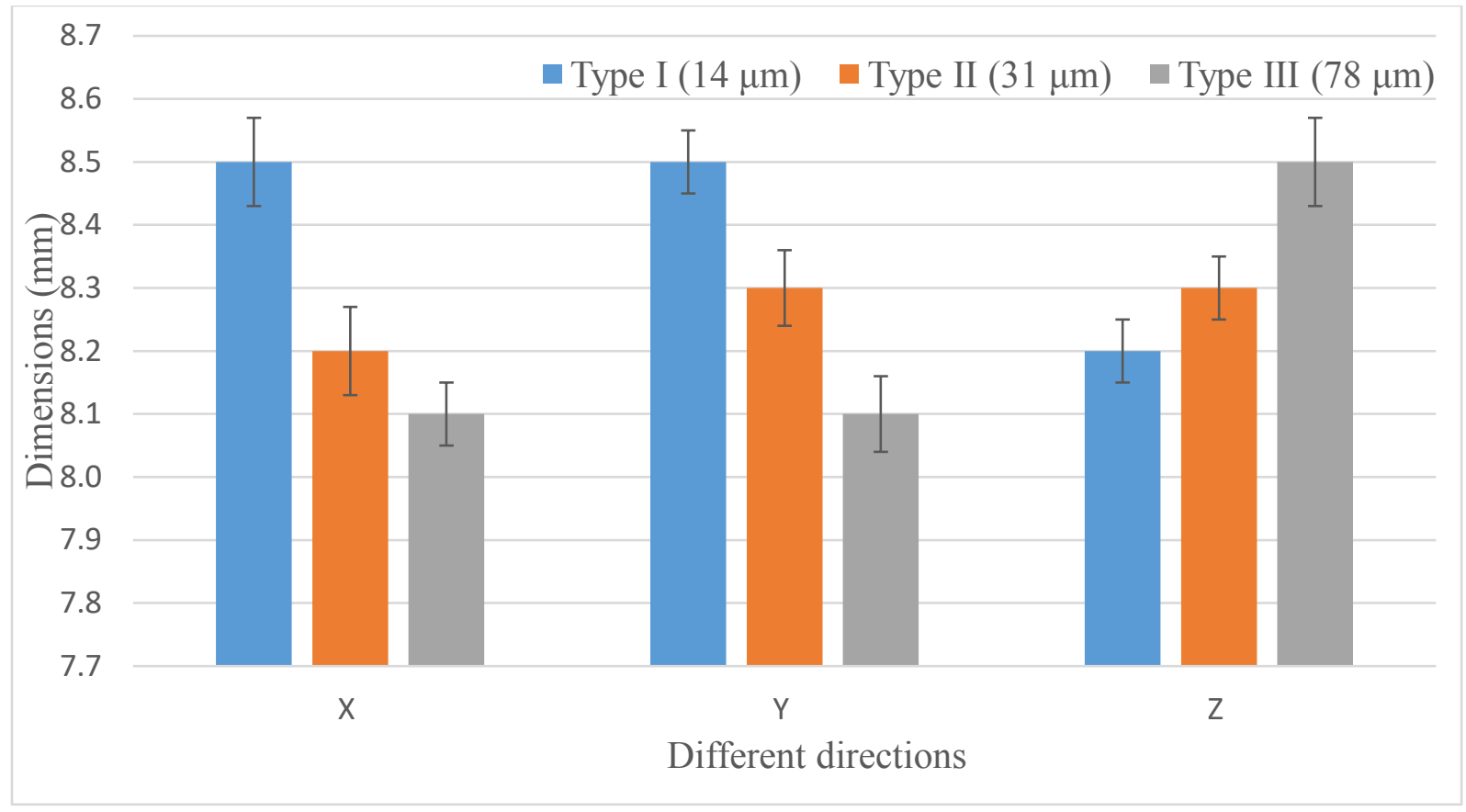

Fig. 6.8 The effect of mean particle size on dimensional accuracy of printed parts

\subsubsection{Surface roughness}

Surface roughness of green parts

To evaluate the influence of the powder particle size characteristics on the surface quality of printed structures, the same cubic samples used for dimensional studies were investigated. The roughness of top surfaces of the samples in different directions, namely $\mathrm{Y}$ (printing direction) and $\mathrm{X}$ (normal to printing direction) as specified in Fig. 6.9, were determined. The results are shown in Fig. 6.10. 


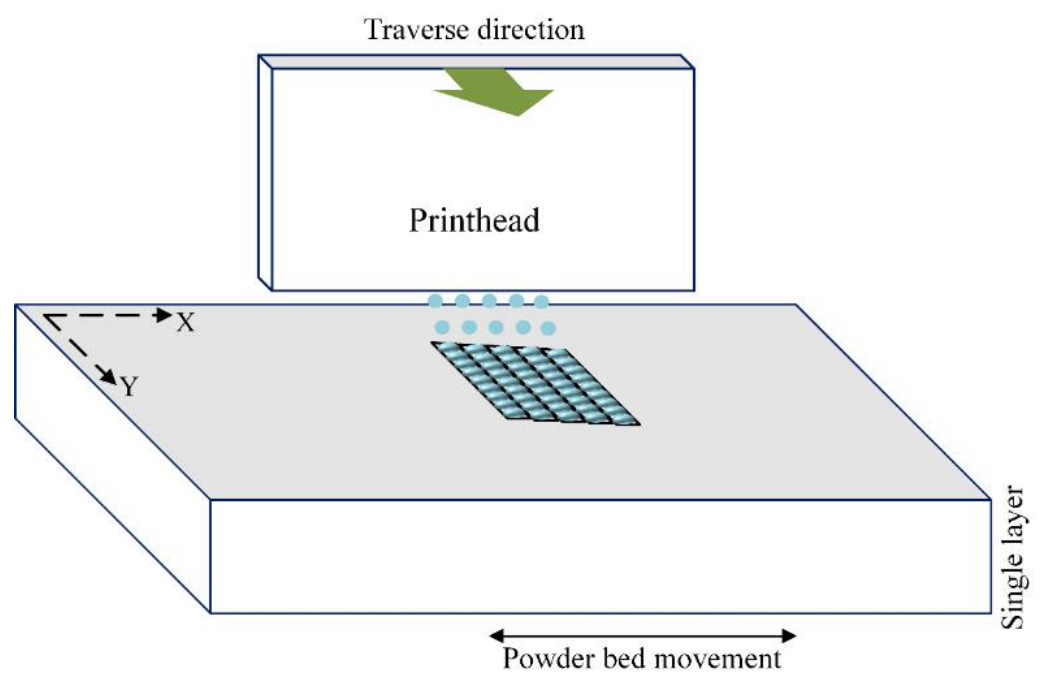

Fig. 6.9 Schematic representation of fabrication platform and defined directions

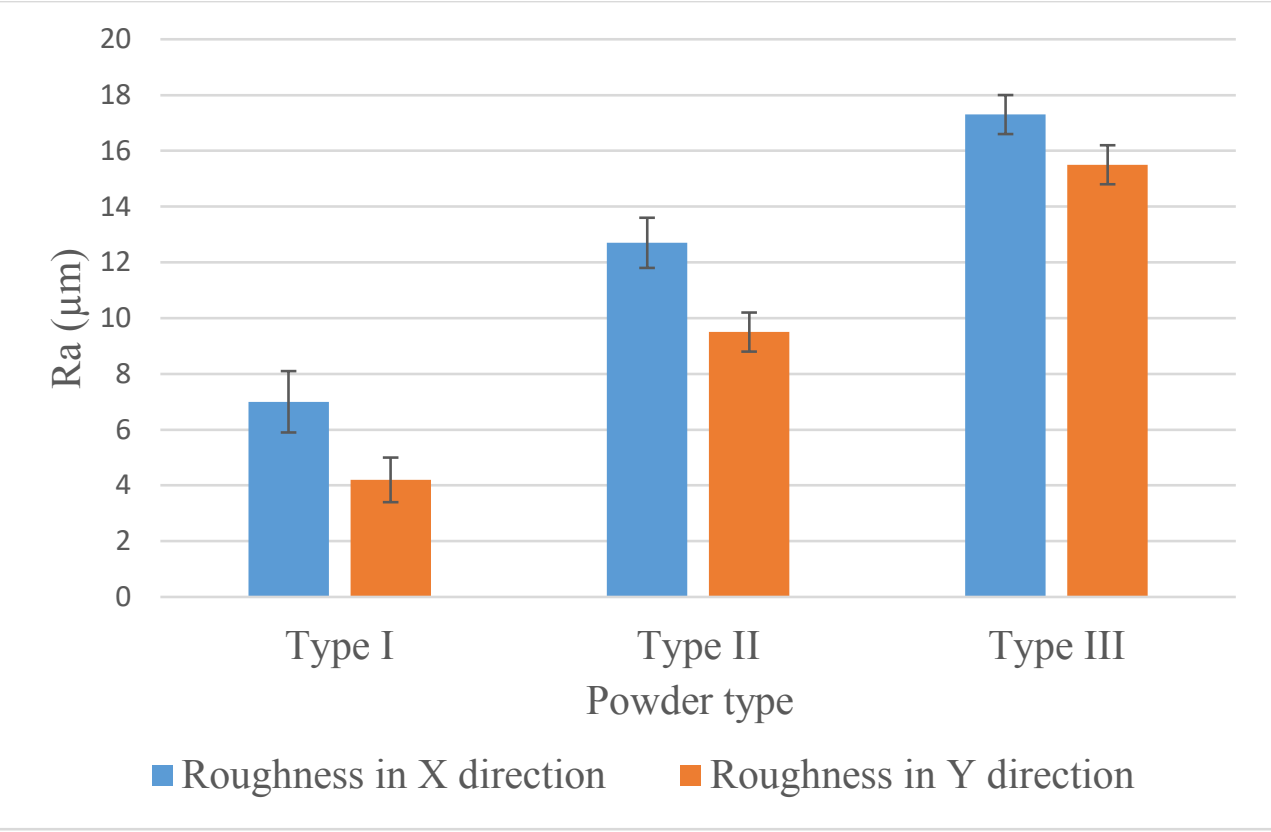

Fig. 6.10 Surface roughness of fabricated green specimens in $\mathrm{X}$ and $\mathrm{Y}$ directions

From the results, the average surface roughness of fabricated specimens in both directions increases with increasing mean particle size. This could be readily attributed to the increased peak-to-valley ranges in powder beds formed by large particle sizes $[163$, 179]. Also, from the results it appears that $R_{a}$ values in $X$ direction are slightly higher than those in Y direction. Such discrepancies in roughness values might be contributed by both 
the binder deposition strategy used in the M-Lab printer and the rearrangement of powder particles from the binder-powder interactions as well as the roller powder spreading mechanism. In the M-Lab printer, droplet delivery to the layered powder bed is carried out in a pattern of raster scanning with droplet impact velocity of around $8 \mathrm{~m} / \mathrm{sec}$ [166]. Considering the common droplet jetting frequency $(4 \mathrm{kHz})$, the droplet velocity and the prinrhead distance from the powder bed surface (approximately $500 \mu \mathrm{m}$ ), the time interval between the successive droplets impacting the powder bed is on the order of a few hundred micro-seconds. As the time for binder droplet depletion from the powder bed surface is on the order of milliseconds [91, 95], on can expect that the droplets could coalesce and form continuous lines before the binder permeation into the powder bed is complete. Therefore, the generation of each cross section of an object in the BJ-AM process can be effectively treated as the formation of straight lines with various lengths as shown schematically in Fig. 6.11.

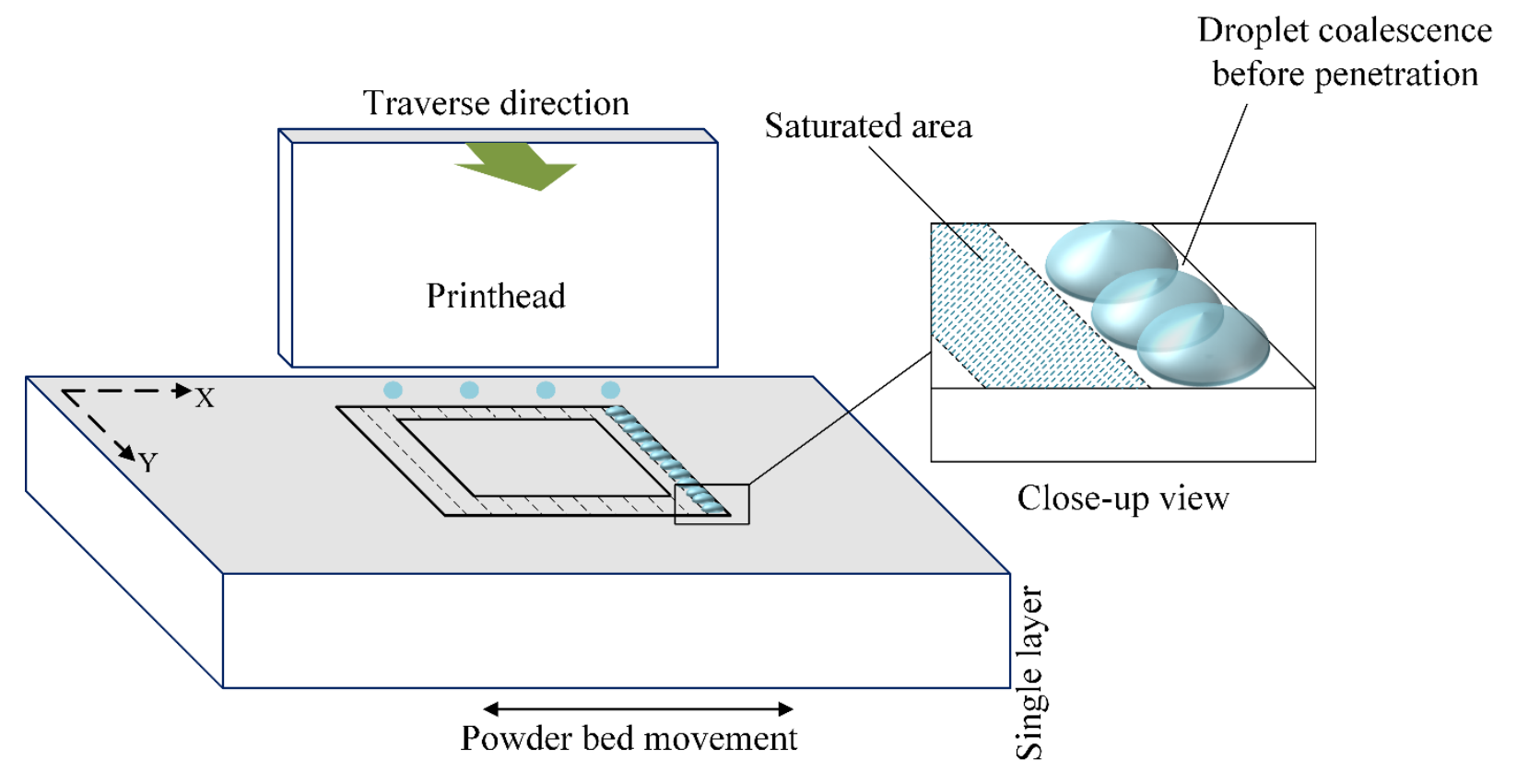

Fig. 6.11 Schematic of $2 \mathrm{D}$ feature formation within a layer 
The formation of such lines before complete binder penetration and the consequent interactions between these lines and the powder bed might result in a more corrugated texture in the $\mathrm{X}$ direction. Such distinctive texture of the surface is also expected to diminish with the increase of mean particle size or when the droplet size (50 $\mu \mathrm{m}$ diameter) becomes comparable to the mean particle size. With larger particle sizes or smaller droplets, the time for droplet complete penetration into the powder bed decreases which in turn would prevent the coalesce of the binder droplets. In order to investigate the "scanning line" effect, cubic containers were fabricated with different powder types and analyzed using the optical microscopy. Fig. 6.12 displays two cubic containers in the green state printed with the powder types I and III. From the images, it is clear that for powder with smaller particle size (Type I), tracks and valleys are visibly discernable along the printhead raster direction. With larger mean particle size (Type III), the effect of the" scanning line" becomes less significant. 

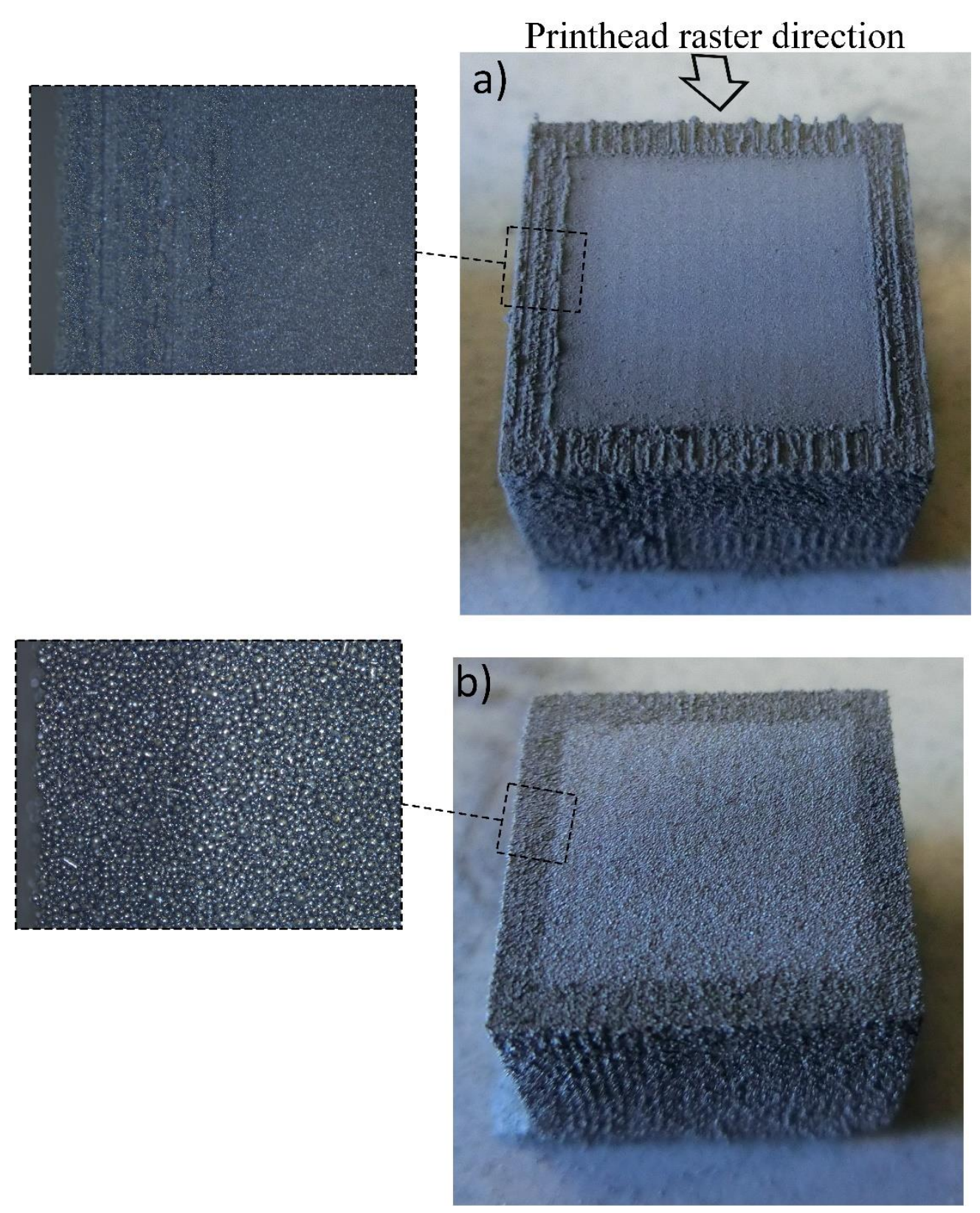

Fig. 6.12 The effect of the raster scanning strategy for binder delivery on the surface roughness of the green parts a) sample fabricated with the powder type I b) sample made from the powder type III

Surface roughness of sintered parts

The surface roughness of the sintered specimens fabricated from powders of different particle sizes is shown in Fig. 6.13. The surface roughness of the samples increases when the mean particle size of the powder material increases, which is consistent 
with the trend observed from the green parts. The parts fabricated with the powder type I exhibited the lowest value of $\mathrm{Ra}$ in both $\mathrm{X}$ and $\mathrm{Y}$ directions. No consistent trend was observed for the roughness values between the un-sintered and sintered groups. On the other hand, under the sintering parameters used in this study, it appears that the discrepancy of the surface roughness between $\mathrm{X}$ and $\mathrm{Y}$ directions was retained after post processing.

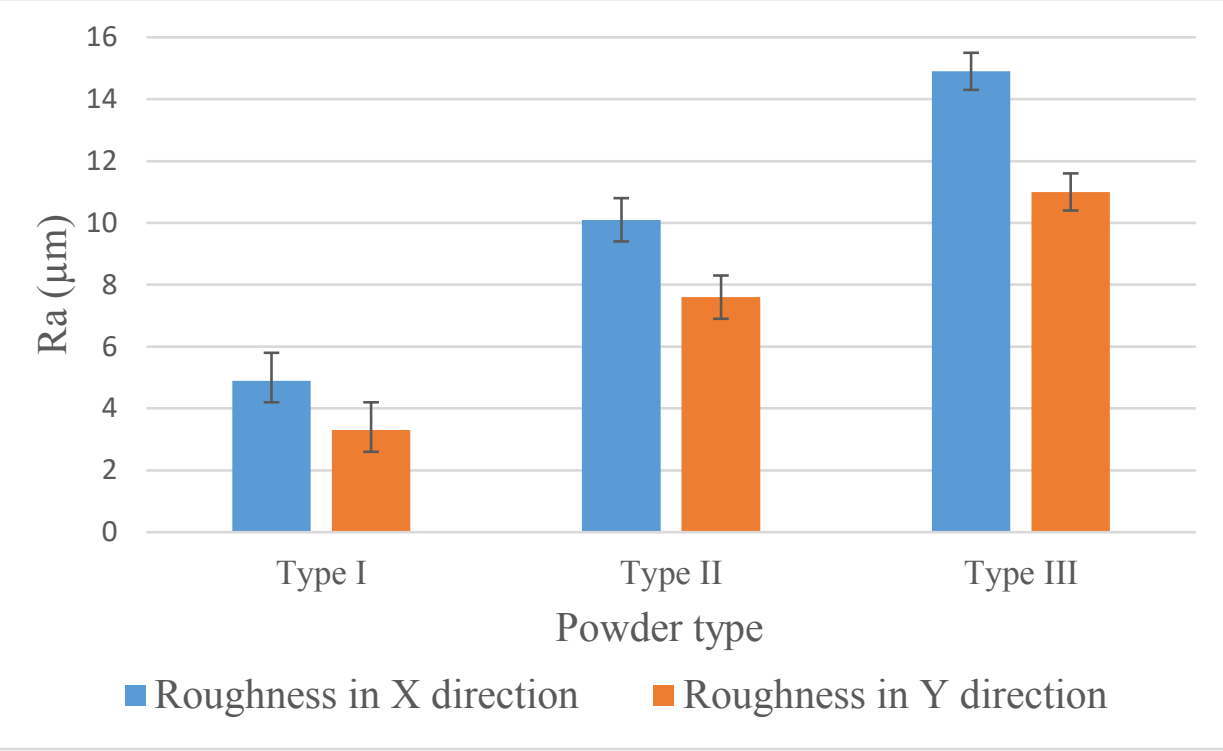

Fig. 6.13 Surface roughness of specimens after sintering in $\mathrm{X}$ and $\mathrm{Y}$ directions

\subsubsection{Density}

\section{Green part density}

In order to compare the results, the measured density of the parts is normalized by the density of the solid $316 \mathrm{~L}$ stainless steel $(7.99 \mathrm{~g} / \mathrm{cm} 3)$. The results of the green part density measurements are shown in Fig. 6.14. From the results, larger mean particle sizes correspond to higher densities of the green parts, which is consistent to the trend observed from the powder bed packing density. It appears that the feature fabrication process does not significantly influence the packing density of the powders. In other words, the droplet impact and the interactive forces involved in the binder penetration into the powder 
material do not appear to impose considerable density change during the fabrication process.

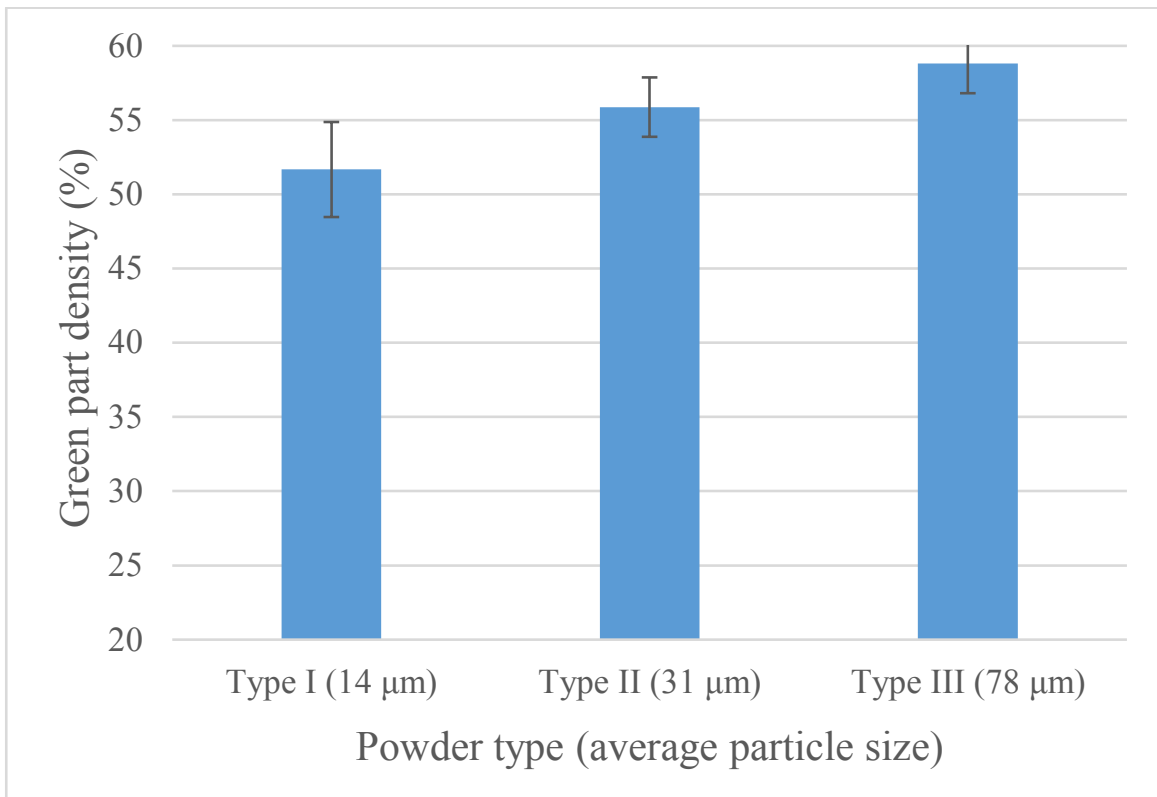

Fig. 6.14 Effect of particle size on green part density

Sintered part density

The effect of the different powder particle sizes on the densities of the sintered parts is shown in Fig. 6.15. The parts fabricated from the powder type I with the smallest mean particle size exhibit the highest density levels (80\%) after sintering. Samples printed from powder type II exhibit similar density levels (78\%) to those made from powder type I. On the other hand, the sintered density of the samples fabricated from powder type III exhibit the lowest density levels (72\%). Although the density of the powder-based parts after the sintering are influenced by their green densities [180], the results from this study suggest that the higher green density does not necessarily lead to higher density after sintering. Similar results based upon experimental, numerical, and analytical investigations have also been reported in the literature [181]. This can be explained by the fact that the powder with larger particle sizes requires higher level of sintering kinetic energy to achieve the same 
level of sintering in comparison to the powder with small particle sizes due to smaller surface to volume ratios and larger boundary radius [182, 183]. At constant sintering temperature of $1400{ }^{\circ} \mathrm{C}$ used in this study, parts fabricated with small particle sizes exhibit higher densities and more homogeneous microstructures as shown in Fig. 6.16. The aspolished cross sections of the sintered samples are shown in Fig. 6.16. The microstructures of the post-processed samples are consistent with the results of the density measurements of the sintered samples (Fig. 6.15). SEM micrograph of the sample made from powder type I (Fig. 6.16 a) shows more uniform microstructure and less porosity. In addition to the smaller particle size, the narrower particle size distribution of this powder type can also lead to narrower pore size distribution and consequently contribute to the homogeneity of the microstructure [184]. As the mean particle size increases, the percentage and the size of the porosities in the microstructure of the sintered samples exhibit an increasing trend.

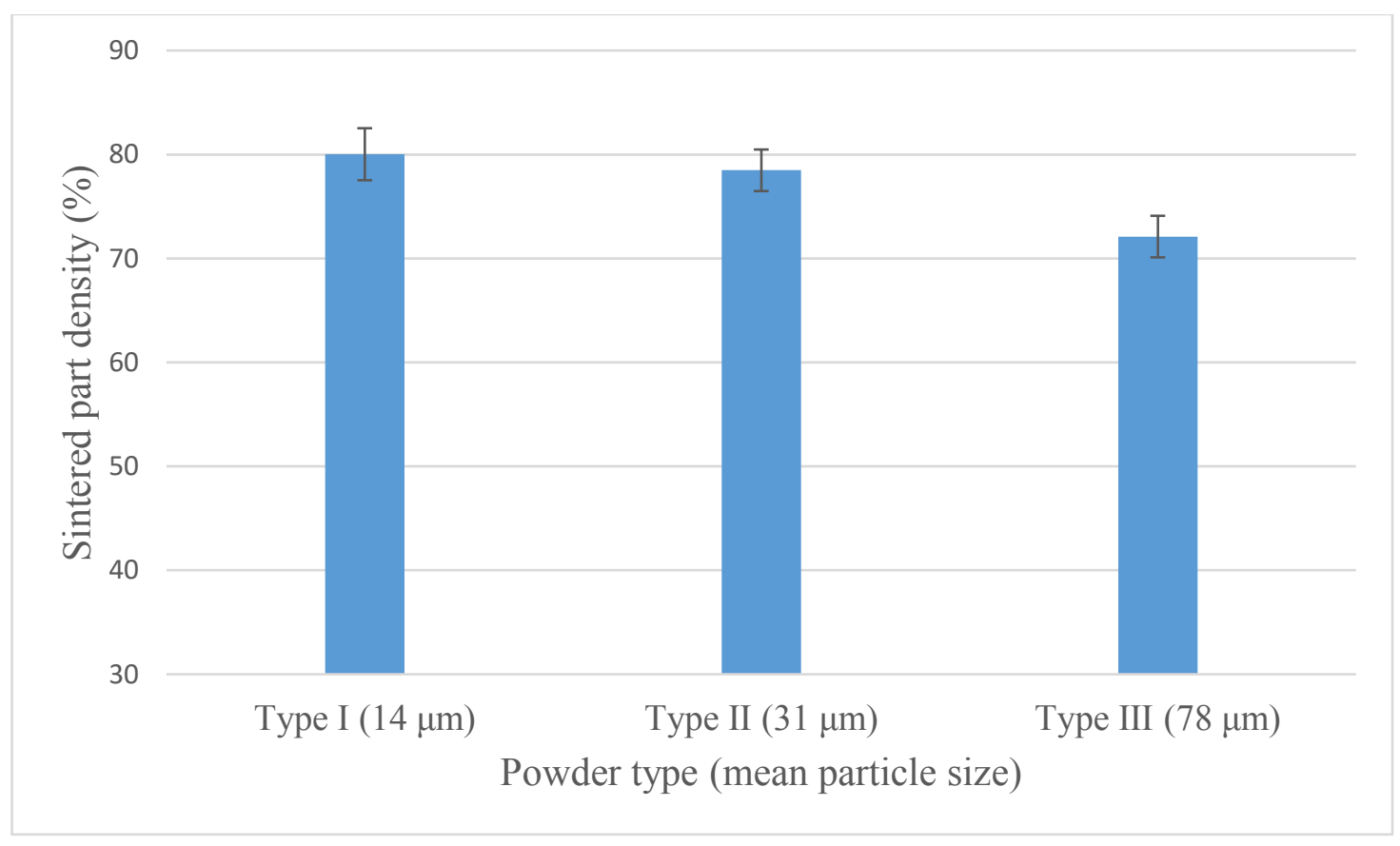

Fig. 6.15 Effect of particle size distribution on the density of the sintered parts 


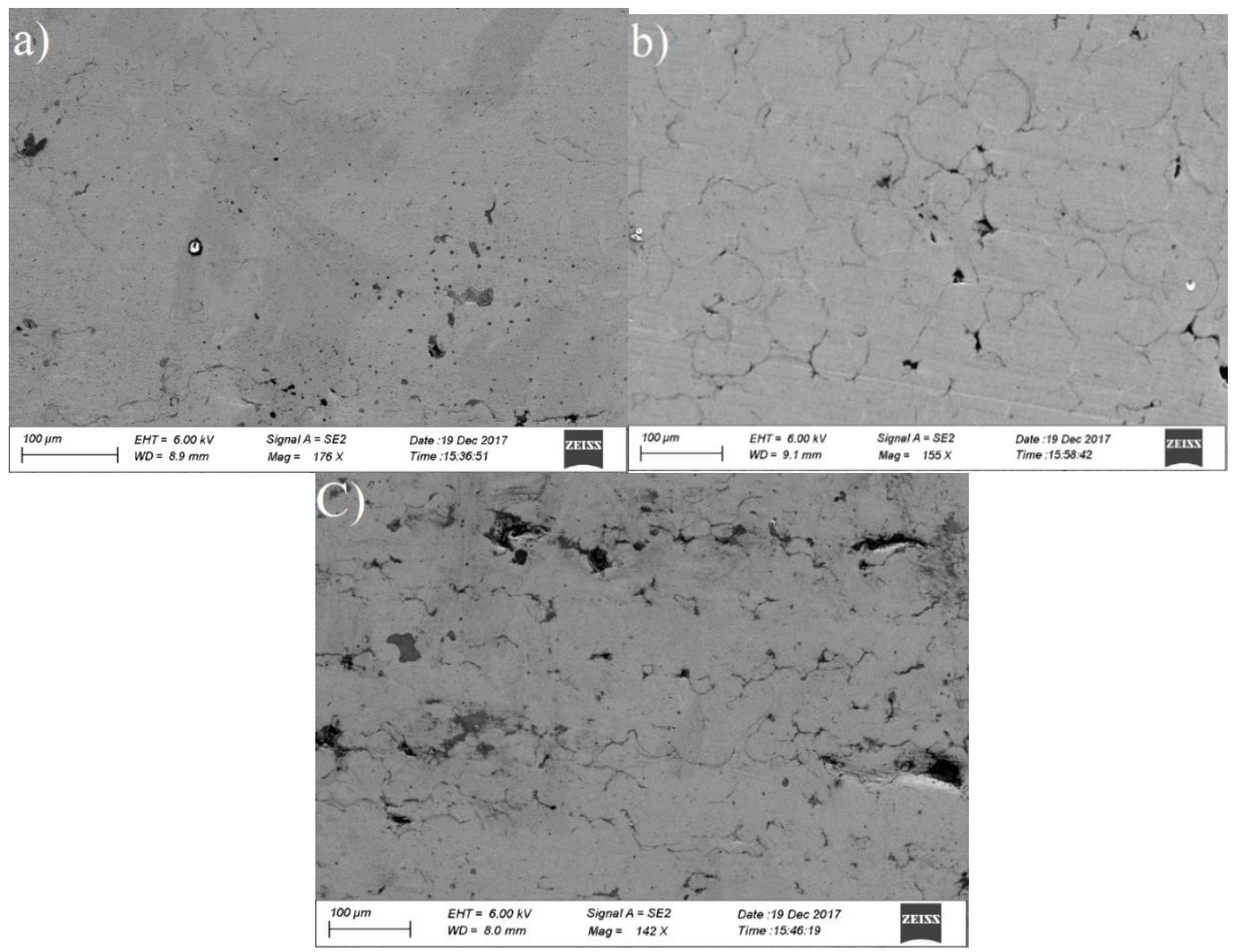

Fig. 6.16 SEM micrographs of sintered specimens a) type I b) type II c) type III

\subsubsection{Microhardness of the sintered parts}

The microhardness of the samples fabricated from different type powder materials after sintering is shown in Fig. 6.17. The parts made with the smaller mean particle size exhibit higher Vickers hardness. Within the range of the powder particle sizes investigated in the present study, as the particle size of the powder materials increases, the hardness of the sintered specimens declines. The powder type III with the largest mean particle size results in the lowest hardness value of $36 \mathrm{HV}$. Such trend is consistent with the results from the density analysis with the sintered samples, which can be readily explained by the porosity characteristics previously discussed. It is generally recognized that the mechanical strength of sintered powder metallurgy parts such as hardness increases as the microstructural porosity reduces [185-187]. Even though powder with larger particle sizes 
might be favorable for higher geometrical accuracy for the green parts, they are likely to result in lower overall qualities with the sintered parts.

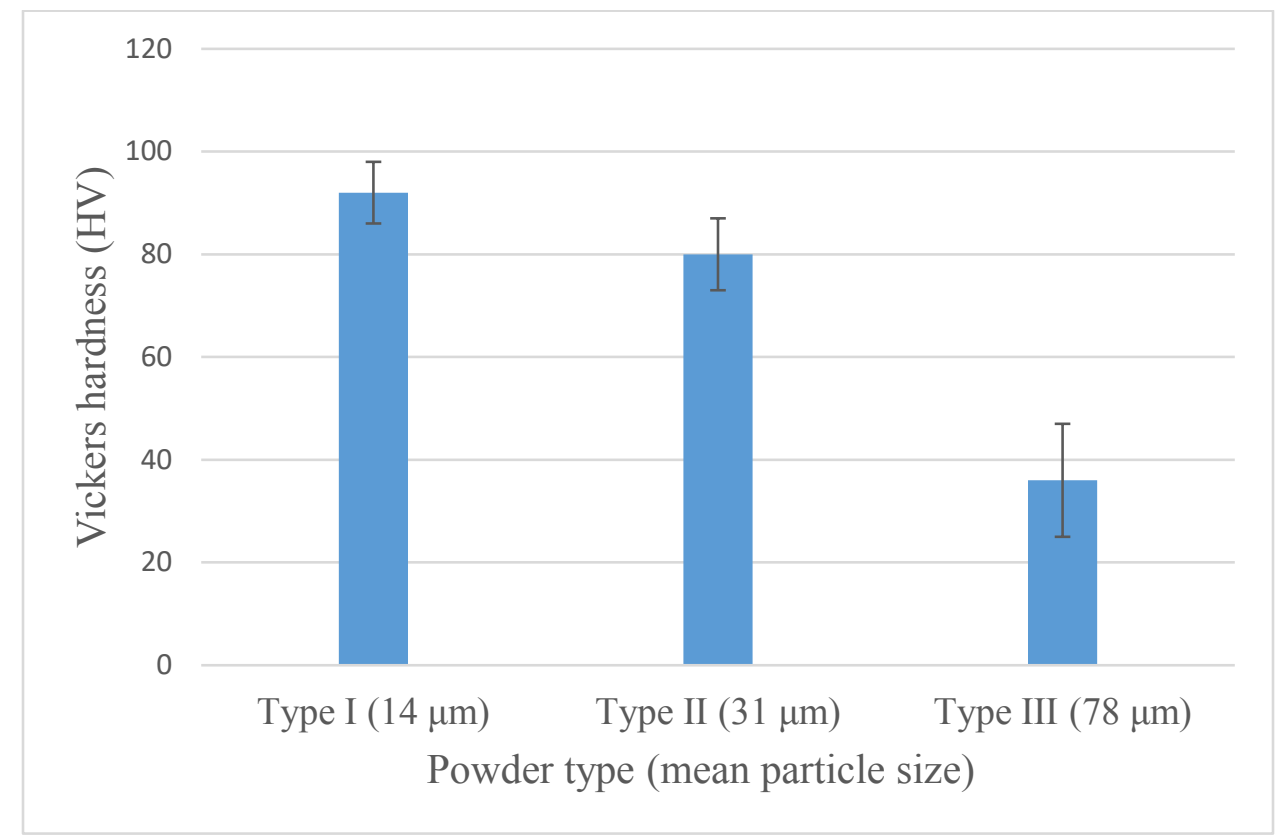

Fig. 6.17 Effect of the mean particle size on hardness of the sintered samples

\subsection{Conclusion}

In the present study, the effect of the powder particle size characteristics on the quality of both the green and sintered parts fabricated via the binder jetting additive manufacturing $(\mathrm{BJ}-\mathrm{AM})$ process was experimentally investigated. The $316 \mathrm{~L}$ stainless steel powder material with three different mean particle size types were used for the experimentation. The results are summarized as follows.

1. The broader particle size distribution resulted in higher powder bed packing densities; 
2. The flow dynamics of the binder droplets including spreading and penetration is significantly influenced by the powder particle sizes, which in turn affects the dimensional accuracies of the printed green parts.

3. Larger powder particle size appears to be beneficial to dimensional accuracies and green part densities of the BJ-AM parts;

4. Smaller powder particle size appears to be beneficial to the surface finish of the fabricated parts, as well as higher mechanical properties and densities of the sintered parts. 


\section{CHAPTER 7}

\section{BINDER DROPLET PROFILE PREDICTION AFTER COMPLETE PENETRATION INTO A POWDER BED}

\subsection{Introduction}

In the binder jetting additive manufacturing process (BJ-AM), each layer of the part geometry is fabricated by selectively applying droplets of a liquid binder on the surface of a layered powder material through a drop-on-demand (DOD) printhead. The deposited liquid binder diffuses into the powder bed and binds powder particles in the regions specified from a CAD model. The entire surface of the layer including saturated and dry areas is then exposed to external heating performed via a radiation heat source in order to partially cure the binder and ensure adequate mechanical strength for consequent printing processes. After the drying/curing, a new layer of the powder will be added to the already printed layer, and the process repeats until the entire green part is printed [100]. The droplets employed in BJ-AM for fabrication of each layer are deposited in a pattern of raster scanning. Each individual droplet printed into a powder bed will create a profile after the interaction of a binder droplet and powder bed is complete. In raster scanning binder delivery pattern, the droplets are deposited consecutively with certain lateral spacing to ensure that such profiles have sufficient overlapping areas to form into a 2D structure 
within each layer. By stacking of the $2 \mathrm{D}$ structures with appropriate binding strength between successive layers 3D geometry of an object is fabricated. Therefore, the droplet profile characteristics as the primitive building element of every 3D printed object are of significant importance in determining the final part quality in terms of mechanical performance and dimensional accuracy. The goal of this chapter is to provide a predictive model for droplet-powder interaction in an effort to estimate the droplet profile characteristics inside the powder bed. To accomplish this objective, the interaction of the binder droplet and powder bed at every stage of droplet evolution from the moment of impact to equilibrium state, where the interaction is complete, needs to be carefully analyzed. In this study, it is assumed that the droplet profile after complete penetration into a power material is of a spherical cap with the radius and height of $\mathrm{r}$ and $\mathrm{h}$, respectively. Therefore, the developed model is to predict the diameter (i.e. maximum droplet spreading) and the height of such cap (the droplet penetration depth) in order to characterize the droplet profile inside any given powder material.

\subsection{Modeling}

The droplets impinging the powder bed surface with a certain velocity might spread, splash, or rebound off the target surfaces upon the impact. The behavior of the impinging droplets on a surface depends on various parameters including droplet physical properties, powder bed physical characteristics, as well as powder bed wettability with the impinging droplet $[113,141,143-145]$. In the binder jetting process, the binder and powder material system is normally chosen in a way that the splashing or rebound phenomenon is less likely to take place due to their respective physical properties and the powder material 
wettability to the liquid binder $[86,158]$. Therefore, the splashing and rebound of an impinging droplet and its effect on the binder/powder bed interaction is not within the scope of the current study. After the impact, the droplets might undergo different phases driven by inertial forces, capillary forces, and gravitational forces $[86,158]$. Due to the droplet volume range and binder physical properties typically used in the binder jetting process, which result in the small Bond number $\left(\mathrm{B}_{O}<<1\right)$, the effects of gravitational forces on the droplet dynamics can be ignored $[86,91,95,158]$. The dimensionless Bond number can be determined from the below equation:

$$
B_{O}=\frac{\rho g L^{2}}{\gamma}
$$

where $\rho$ is density of the drop, g the acceleration due to gravity, $\mathrm{L}$ is taken to be diameter of the drop before impact and $\gamma$ is the surface tension. For the BJ-AM system examined in the present investigation, the resulting Bond number is on the order of $10^{-4}$, which indicates that gravity influence on the droplet interaction with powder bed is negligible. Therefore, inertial and capillary forces can be considered as the dominant driving forces for spreading and penetration of liquid binder droplets into the powder bed [91, 158]. From a physiochemical perspective, the interaction between a liquid droplet and a porous material is a very complex process [188]. In general, the drop dynamics on impact can be divided into impact dominant phase and capillary dominant phase. While in the impact dominant phase the droplet initial velocity is important, and the inertial forces govern the spreading dynamics of the droplet, in the latter phase the capillarity becomes the dominating mechanism for the droplet behavior $[113,158,189]$. It has been shown by various researchers that these two phases occur at different timescales and as a result, might be analyzed separately $[91,158,189]$. As a droplet impacts with the surface, initial impact 
spreading in which the contact line between the droplet and the target surface expands radially starts that is mainly derived by the initial kinematic energy. The degree of spreading over the surface at this stage depends not only on the binder and powder material physical properties but also on the compact conditions and surface wettability. During this phase and before the capillary driven flow occurs, the droplet might experience some momentary disturbances or damped oscillation of the shape [86]. Toward the end of the impact dominating phase, the capillary forces overshadow the inertial forces and begin to dominate the droplet flow dynamics. At this stage, droplet behavior is largely controlled by capillarity and further expansion of the contact line might occur before droplet penetration into the powder bed is complete $[86,95,113,188,190]$.

The impact driven spreading has been investigated by many researchers. There exist numerous experimental, theoretical, and numerical studies on the maximum spreading radius (or diameter) of an impinging droplet mostly on solid surfaces due to its technological importance in various industries $[91,113,144,154,189,191]$. In the literature, the theoretical models that are derived from energy conservation have been widely used for predicting the maximum spreading diameter because of the simplicity and accurate predictive capabilities of these models [189]. There exist several analytical models based on energy balance approach that can be used for prediction of the maximum spreading diameter such as Mao et al. [147], Fukai et al. [192], Collings et al. [193], Chandra and Avedisian [194], Pasandideh-Fard et al. [195], Park et al. [196], and Ukiwe et al. [197]. Among these works, the model developed by Park et al. [196] has been shown to predict the maximum spreading diameter with greater accuracy under the conditions which are representative of typical drop-on-demand ink-jet printing [189, 196, 198]. 
Therefore, in the current study the following expression derived from Park et al. model is used to predict the maximum spreading diameter of the binder droplet impinging on the powder bed surface in the absence of capillary penetration.

$$
\beta^{*}=\left(\frac{W e+12+3\left[f_{s}-\cos \theta\left(\frac{4 \sin ^{3} \theta}{\left(2-3 \cos \theta+\cos ^{3} \theta\right)^{\frac{2}{3}}}\right)\right.}{3\left(f_{s}-\cos \theta\right)+\frac{4 W e}{\sqrt{R e}}}\right)^{1 / 2}
$$

where $f_{S}=\frac{2(1-\cos \alpha)}{\sin ^{2} \alpha}, \alpha$ is the contact angle at maximum spreading, $\beta^{*}\left(=\frac{D^{*}}{D_{0}}\right)$ is the maximum spreading factor which is equivalent to the ratio of the maximum spreading diameter $\left(D^{*}\right)$ to initial droplet diameter before impact $\left(D_{0}\right)$, We $\left(=\rho D_{0} V_{0}^{2} / \sigma\right)$ is dimensionless Weber number, $\operatorname{Re}\left(=\rho D_{0} V_{0} / \eta\right)$ is the dimensionless Reynolds number, $\theta$ is the equilibrium contact angle between the liquid binder and the surface, $\rho$ is the liquid density, $V_{0}$ is the impact velocity, $\sigma$ is surface tension, and $\eta$ is liquid viscosity.

At end of the initial impact driven phase and after reaching the maximum diameter, as the capillary forces begin to dominate the interaction between the droplet and powder, two scenarios might occur depending on the rate of the droplet vertical penetration into the powder bed $\left(\mathrm{V}_{\mathrm{P}}\right)$. It is worth mentioning that the penetration rate of a deposited droplet on a porous surface is largely determined by the liquid physical properties, powder bed characteristics, and powder material wettability to the impinging droplet $[53,86,95,199]$. In the first scenario, the rate of vertical penetration of the droplet into the powder bed is sufficiently high that droplet spreading over the surface is inhibited due to the quick depletion of the droplet from the powder bed surface. In other words, rapid penetration of the droplet into the porous bed impedes the capillary spreading of the droplet over surface. Another possible scenario is the further extension of the contact line between the droplet 
and the powder bed surface due to the simultaneous vertical penetration and lateral spreading over the surface. In this case, the penetration rate is comparable to that of capillary spreading over the surface and further spreading of the droplet might extend the saturated area in the lateral direction. Fig. 7.1 schematically shows these prospective scenarios which might take place during the capillary driven phase of the droplet and powder bed interaction.
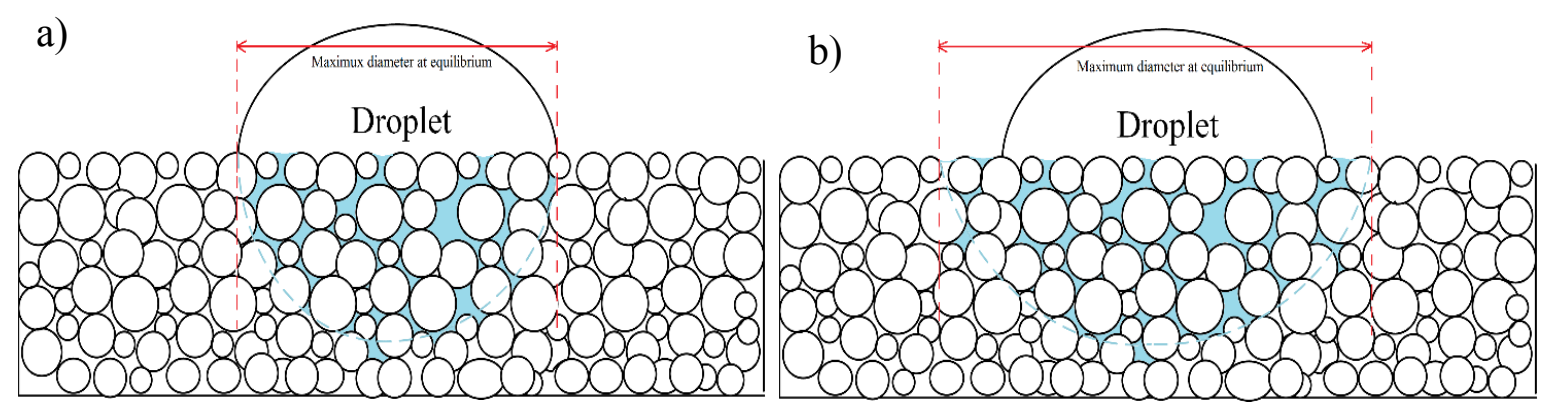

Fig. 7.1 Schematic representation of droplet penetration into the powder bed a) Vertical penetration rate $(\mathrm{VP})>>$ radial spreading rate over the surface (VS) b) VP $\sim$ VS

Fig. 7.2 illustrates the sequence of events that occur after a droplet impacts on a substrate as described previously. 


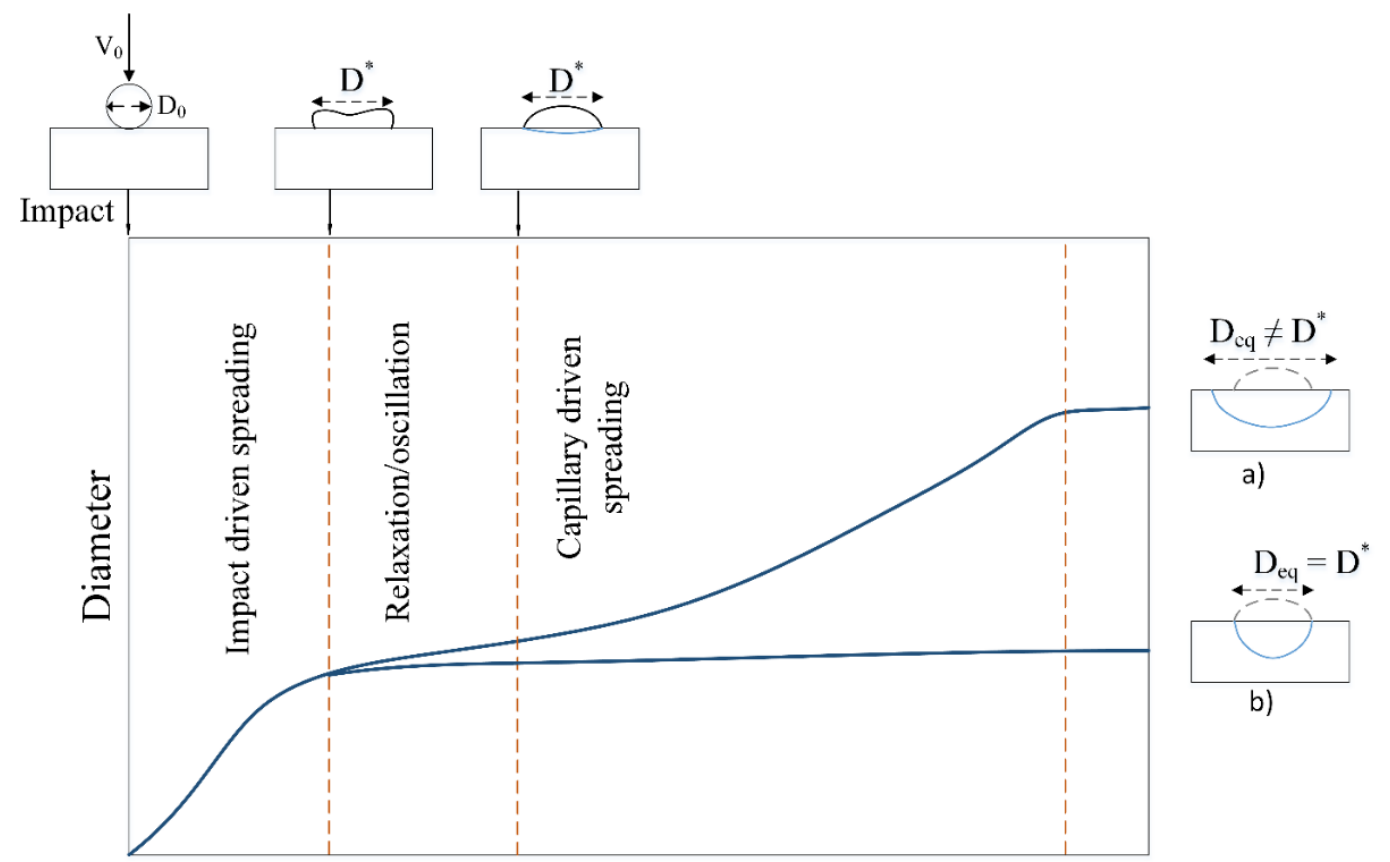

Time

Fig. 7.2 Schematic illustration of the sequence of events that occurs after a droplet impacts on a porous substrate a) Vertical penetration rate (VP) $>$ > radial spreading rate over the surface (VS) b) VP VS

For both scenarios, the maximum spreading radius (or diameter) could be determined from the fact that at one point during the binder/powder material interaction the droplet remaining on the powder bed surface reaches an equilibrium state before complete migration into the powder bed. The diameter of the droplet at this equilibrium state corresponds to the maximum spreading extension of interest and can be found as follows.

The total penetrated volume at time $t, V_{p}(t)$, can be determined from the model presented by Denesuk et al. [53] according to the following equation:

$$
V_{p}(t)=\frac{K}{2} \int_{0}^{t} \frac{r^{2}(t)}{\sqrt{t}} d t
$$


Where

$$
K=\pi \cdot a_{p} \sqrt{\frac{\gamma \cdot \cos \theta \cdot R_{\text {pore }}}{2 \eta}}
$$

$r(t)$ is the instantaneous radius of the spreading droplet at time $t, R_{\text {pore }}$ is the pore radius (mean pore radius from the BET measurements), $a_{p}$ is the volume fraction of the pores, $\gamma$ is the surface tension of the liquid, $\theta$ is contact angle of the binder droplet on the material, and $\eta$ is the droplet liquid viscosity. From equation (7-3), in order to obtain $V_{p}(t)$ (i.e. the droplet volume penetrated into a porous material at any given time) $r(t)$ needs to be initially determined.

The radius of a small droplet spreading on a solid is typically described by the following equation $[95,200,201]$.

$$
r(t)=Q \cdot t^{n}
$$

Where $\mathrm{r}$ is the spreading droplet radius and $\mathrm{Q}, \mathrm{n}$ are constants. From this correlation, time zero $(\mathrm{t}=0)$ clearly results in a radius of zero. However, as in this study the time 0 is taken to the be the point at which the capillary spreading begins, $r(0)$ is clearly of a finite value due to the initial impact driven spreading. Therefore, the following equation with non-zero starting radius was adopted to be used in Eq. (7-3) [95, 202].

$$
r(t)=a \cdot(b+t)^{n}
$$

Where $\mathrm{a}$ and $\mathrm{b}$ are constants, and $\mathrm{n}$ is the same as in Eq. (7-5). In the literature, $\mathrm{a}$ broad range of values have been reported for $\mathrm{n}$, from 0.033 to 0.316 , for different material 
systems [95, 200, 201, 203]. It is well known that the value of $n$ depends on temperature, humidity, $\mathrm{pH}$, the presence of impurities or electrolytes, polymers, surface charges, etc. [202-204].

$\mathrm{a}, \mathrm{b}$, and $\mathrm{n}$ values for each material system can be experimentally determined by real-time imaging of a droplet interaction with any porous/non-porous material taken from the moment of impact to the equilibrium state. Furthermore, $a_{p}, \gamma, \theta, R_{p o r e}, \eta$ need to be experimentally measured in order to calculate the value of $\mathrm{K}$. With all the required inputs in Eq. (7-4), the volume of the droplet remaining on the powder bed surface at any given time can be calculated from Eq. (7-7),

$$
\mathrm{V}_{\mathrm{r}}(\mathrm{t})=\mathrm{V}_{0}-\mathrm{V}_{\mathrm{p}}(\mathrm{t})
$$

Where $V_{0}$ is the droplet initial volume before the impact and $V_{r}(t)$ is the volume of the droplet remaining on the powder bed surface at any given time.

After the impact and in the capillary driven phase, the contact angle between the droplet and powder bed surface undergoes decreasing changes. In other words, the contact angle will pass from the advancing value through the static contact angle at the point at which spreading stops, progressing then to the receding contact angle [95]. For this one instant that the spreading stops, the remaining liquid on the powder bed surface will have the equilibrium form. The diameter of this equilibrium shape which equals to the maximum spreading diameter of interest might be determined applying one of the equations below, Eq. (7-8) or Eq. (7-9) depending on the droplet impact velocity [86, 95].

$$
d_{e}(t)=2 \cdot\left(\frac{3 \cdot V_{r}(t) \cdot \sin ^{3} \theta}{\pi \cdot(1-\cos \theta)^{2} \cdot(2+\cos \theta)}\right)^{1 / 3}
$$




$$
d_{e}(t)=2 \beta^{*} \cdot\left(\frac{3 \cdot V_{r}(t)}{4 . \pi}\right)^{1 / 3}
$$

Eq. (7-8) provides the equilibrium radius of a droplet of a given volume carefully placed on a surface (i.e. negligible impact velocity) in the absence of infiltration which assumes a spherical cap shape at equilibrium. On the other hand, Eq. (7-9) gives the maximum equilibrium radius of a droplet impacting a non-porous surface with a considerable velocity. Using droplet initial volume $\mathrm{V}$ in the equations (7-8) and (7-9), if the droplet impact velocity is not negligible, Eq. (7-9) would result in higher value and should be used in the modeling. Otherwise, Eq. (7-8) should be used for the calculations and would lead to more accurate predictions.

Therefore, by determining the point at which the equilibrium diameter and the diameter of the spreading droplet are equal the maximum spreading diameter of interest can be numerically found.

In addition to the maximum droplet extension (diameter of droplet profile), the depth of the droplet penetrated a powder bed should also be determined for fully predicting the droplet profile inside the powder bed. For this purpose, the Washburn equation can be used according to the equation below.

$$
h=\left[\frac{R_{\text {pore }} \cos \theta}{2}\right]^{\frac{1}{2}}\left[\frac{\gamma}{\eta}\right]^{1 / 2} t^{1 / 2}
$$

Where $\mathrm{t}$ is the time for complete absorption of the binder droplet. Denesuk in [53] has shown that this time can be estimated by the following relation.

$$
t=\frac{\eta R_{\text {pore }}^{3}}{32 \gamma \cos \theta}\left(\frac{V_{0}}{\pi r_{\max }^{2} k}\right)^{2}
$$


Where $r_{\max }$ is the maximum radius of the spreading droplet on a powder bed surface and $\mathrm{k}$ is the powder bed permeability which can be experimentally measured for any material system (i.e. liquid binder and powder material).

7.3 Methodology for the model validation

In order to assess the accuracy of the proposed model, a real-time imaging system using Fastec Imaging HiSpec 2 high speed camera was developed. As shown in Fig. 7.3, the system consists of a micropipette (2-10 microliter droplet volume size), the high-speed camera, mechanical fixtures for holding the micropipette and the camera, and a light source (150W Fiber Optic Dual Gooseneck Microscope Illuminator). Individual droplets of $2 \mu 1$ volume were deposited using the setup, and real-time flow dynamics of the impacting droplets on different powder beds were recorded. The final diameter and depth of the resulting liquid-permeated features were measured by an optical microscope (Olympus MX51 with Olympus QColor 5 digital camera). For all the experiments, the same types of the 316 L Stainless Steel powder as in chapter 6 were used. The ExOne M-Lab printer was used for forming the powder beds with the roller spreading speed of $2 \mathrm{~mm} / \mathrm{s}$. The binder used for the interaction study was the ExOne PM-B-SR1-04, which is an ether solventbased binder compatible with the M-Lab system. 


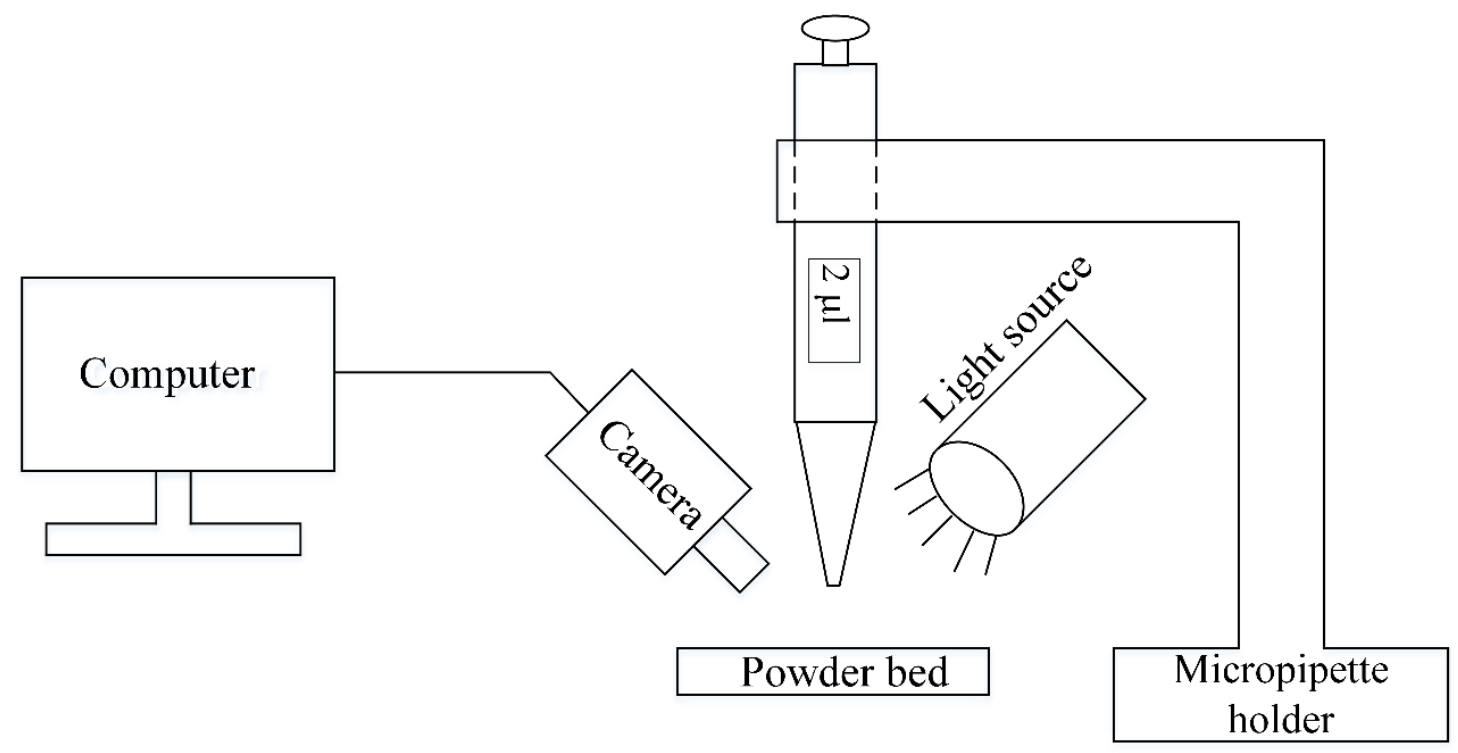

Fig. 7.3 The schematic of high-speed imaging setup

The Cannon-Fenske Routine Viscometer, size 150, was employed to measure the viscosity of the liquid binder according to ASTM D445 and ISO 3104 [205]. For determining the surface tension of the binder, stalagmometric method was chosen. In this method, the total weight and number of drops of the liquid binder falling from a capillary glass tube (Fig. 7.4) were recorded, and the surface tension of the fluid were consequently calculated.

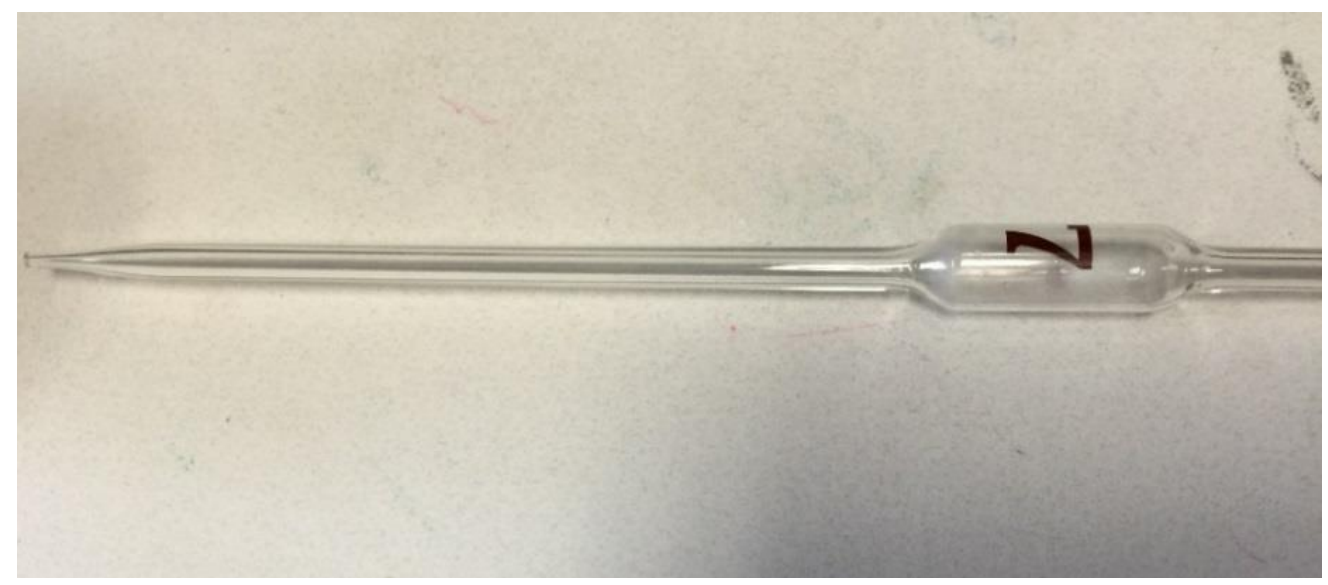

Fig. 7.4 Volumetric pipet used for measuring the surface tension of the binder 
For measuring the permeability of the powder beds, k, a set up shown in Fig. 7.5 was used [24]. The tube is secured in the bottom end and filled with a liquid binder on top of the sample. In this experiment, if the height is measured over time, an equation of the following form can be employed to determine the permeability [24].

$$
\mathrm{h}=\mathrm{h}_{0} \mathrm{e}^{-\mathrm{nkt}}
$$

where $\mathrm{k}$ is the permeability, $\mathrm{L}$ is the sample thickness, $\mathrm{A}$ is the sample cross-section, $\mathrm{r}$ is the inside radius of the tube, and $\mathrm{n}$ is a geometrically determined constant which is defined as

$$
\mathrm{n}=\mathrm{A} / \pi \mathrm{r}^{2} \mathrm{~L}
$$

where $\mathrm{h}_{0}$ corresponds to the initial height of binder at time 0 . The permeability can be found by fitting a line to the collected data plotted as $\ln \left(\mathrm{h} / \mathrm{h}_{0}\right) \mathrm{vs} \mathrm{t}$.

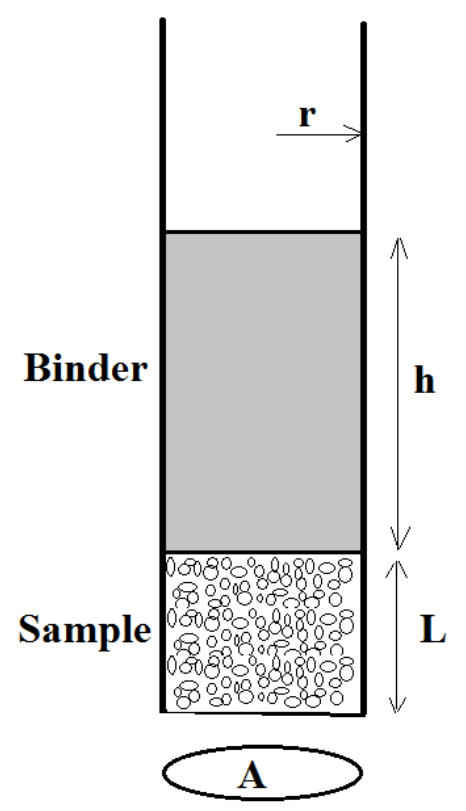

Fig. 7.5 Schematic diagram of the set up for permeability measurement 
To measure the packing density of the powder beds, rectangular cups with the inner dimensions of $30 * 50 * 20 \mathrm{~mm}$ were printed and retrieved after binder curing at $200{ }^{\circ} \mathrm{C}$ for at least 2 hours to determine the mass of the layered powder and volume occupied inside the cup. Given the bulk density of the material, powder weight and the inner volume of the cup, the packing density of each powder type was calculated. For measuring the dimensional characteristics of the samples, digital caliper, micrometer, and/or the optical microscope were utilized depending on the size and structural integrity of the parts examined.

The contact angle is one of the key parameters which can be empirically determined for any material system. For the present study, the sessile drop method was employed to determine the equilibrium contact angle $(\theta)$ and the contact angle at maximum spreading $(\alpha)$ between the liquid binder and different powders [122, 123]. For this purpose, glass microscope slides $(30 \times 30 \times 2.5 \mathrm{~mm})$ covered with double-sided adhesive tape were shaken briefly in a container of the tested powder, and excess particles which were not firmly attached to the surface were removed by means of low pressure air cleaning $[122,123]$. In order to obtain accurate results, the volume of the binder droplets used for the contact angle measurements must be chosen in such a way that the effect of gravity can be neglected. When the liquid droplet size exceeds a particular length known as the capillary length as defined in Eq. (7-10), gravitational effects become significant [124, 125]:

$$
l=\sqrt{\frac{\gamma}{\rho g}}
$$

Therefore, considering the appropriate droplet diameter obtained from Eq. (7-10) for the liquid binder used by the M-Lab, the $2 \mu \mathrm{l}$ droplets were placed on the glass slides, 
which correspond to $\sim 1.5 \mathrm{~mm}$ droplet diameter that is smaller than the capillary length of the binder $(1.7 \mathrm{~mm})$. Five replicates for each powder material were used for the contact angle measurements, and the average results was used in the calculations.

Furthermore, all the math work for solving the equations was carried out using PTC Mathcad Prime 4.0

\subsection{Results and discussion}

The values of the parameters required for determining the maximum spreading radius in the impact driven phase (or diameter) are given in Table 7.1.

Table 7.1 Experimental parameters needed for impact driven maximum spreading

\begin{tabular}{|c|c|}
\hline Binder density $\left(\mathrm{g} / \mathrm{cm}^{3}\right)$ & 0.99 \\
\hline Impact velocity $(\mathrm{mm} / \mathrm{s})$ & 50 \\
\hline Surface tension $(\mathrm{N} / \mathrm{m})$ & 0.03 \\
\hline Binder viscosity $(\mathrm{Pa} . \mathrm{s})$ & 0.00431 \\
\hline Weber number & 0.13 \\
\hline Reynolds number & 18.3 \\
\hline
\end{tabular}

The experimentally determined contact angles $(\theta$ and $\alpha)$ for different powder beds are also given in Table 7.2.

Table 7.2 Experimentally measured contact angles

\begin{tabular}{|c|c|c|}
\hline Powder grades & Equilibrium contact angle $(\theta)$ & Contact angle at maximum spreading $(\alpha)$ \\
\hline Type I & 30 & 50 \\
\hline Type II & 32 & 58 \\
\hline Type III & 33 & 65 \\
\hline
\end{tabular}


Having the equilibrium contact angle $(\theta)$ and the contact angle at maximum spreading $(\alpha), f_{s}$ and $\beta^{*}$ cane be calculated for each material system. According to the experimentally measured parameters, dimensionless $\beta^{*}$ was determined to be $2.9,2.6$, and 2.4 for the powder beds composed of the 316L SS type I, II, and III, respectively.

Fig. 7.6 shows the diameter of the spreading droplet on the surface of the powder bed of type I as a function of time. In the graph, the time 0 corresponds to the point at which the impact driven phase ends and the spreading due to the capillary forces begins to occur. As previously described, data (instantaneous spreading diameter versus time) in the Fig. 7.6 can be represented by an equation of the type given by Eq. (7-6). Therefore, a curve of this sort with parameters of $1.08,10.48$, and 0.155 for a, $\mathrm{b}$, and $\mathrm{n}$, respectively, was fitted to the data as shown by the red color in Fig. 7.6.

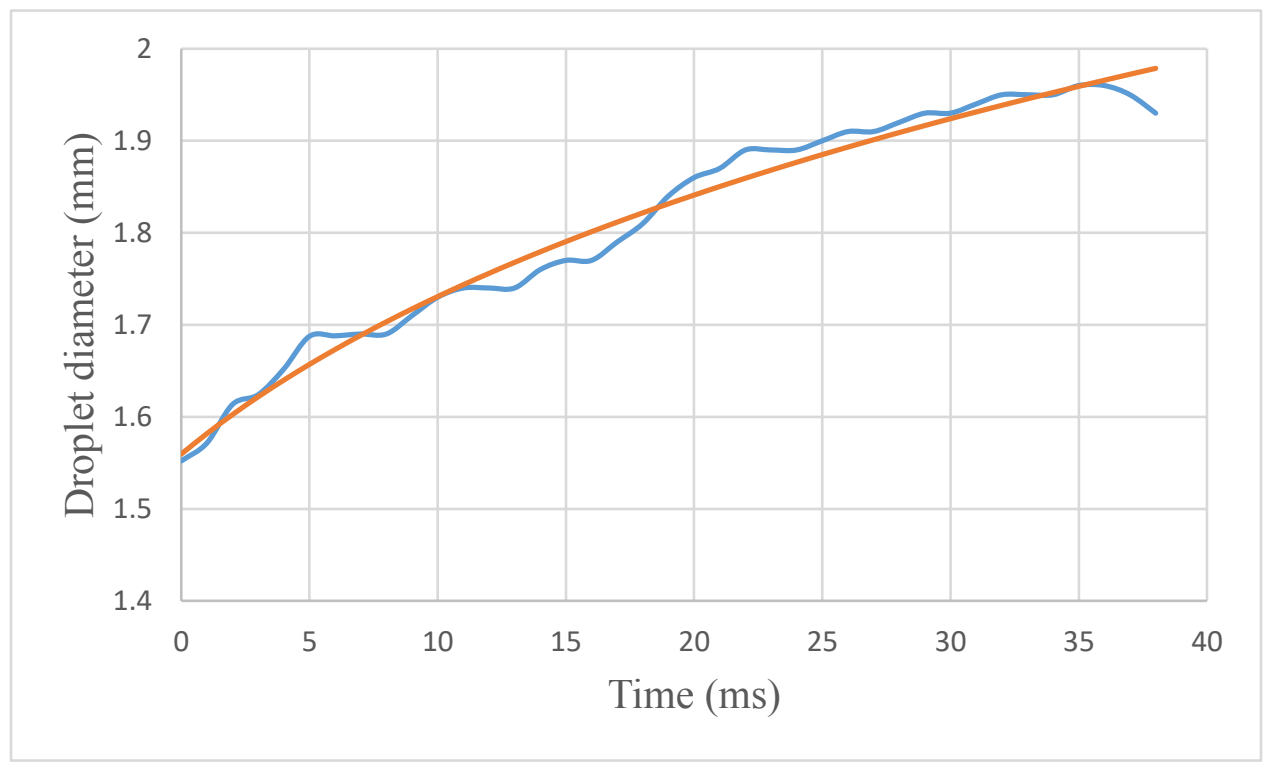

Fig. 7.6 Diameter of a droplet spreading on the surface of different powder beds versus time 
The real time images of characteristic time series of the droplet interaction with powder bed of type I 316L stainless steel material are shown in Fig. 7.7.

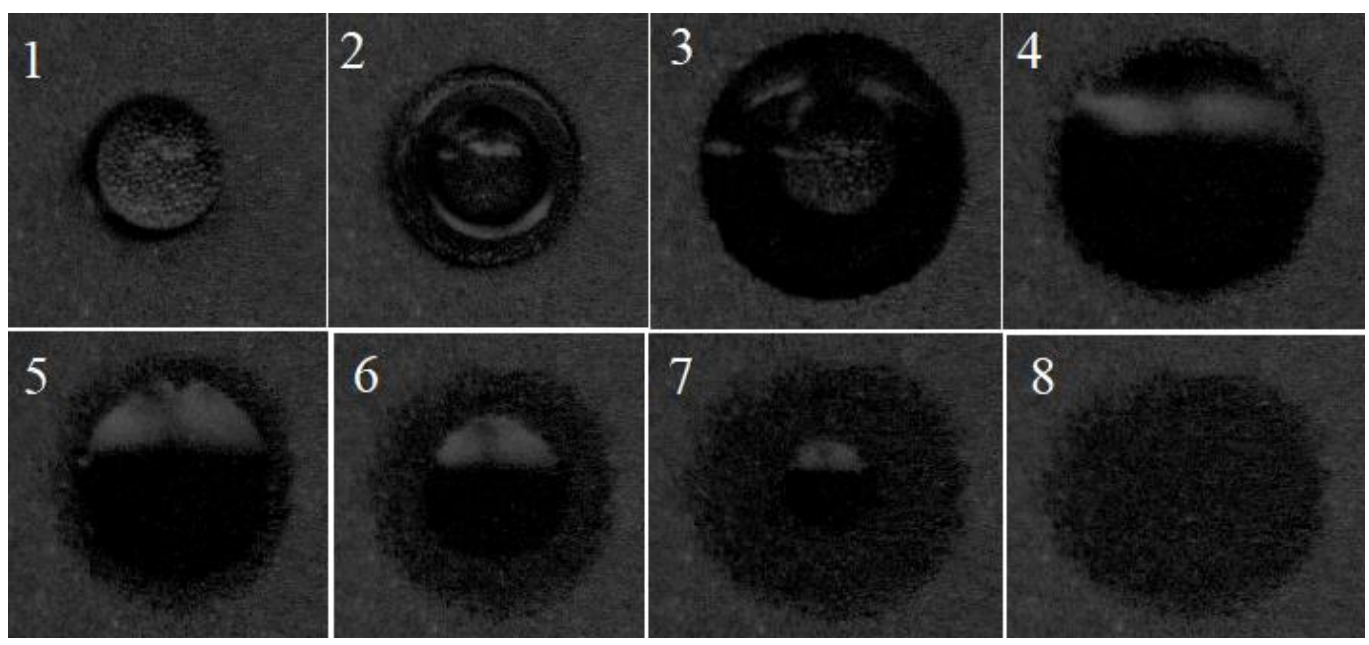

Fig. 7.7 Real time images of characteristics of the droplet interacting with powder material type I from impact (image 1) to complete penetration (image 8)

R-squared of the fit is 0.95 which indicates that the fitted model accounts for 96 percent of the variability of the data. The resulting equation for the instantaneous droplet radius on the surface of type I powder bed is as follows.

$$
r(t)=1.08(10.48+t)^{0.155}
$$

The same procedure was applied for the other powder types (type II and III), and the results given in Table 7.3 were found for the fitting parameters.

Table 7.3 Fitting parameters for Eq. (7-6)

\begin{tabular}{|c|c|c|c|}
\hline Powder grades & $\mathrm{a}$ & $\mathrm{b}$ & $\mathrm{n}$ \\
\hline Type I & 1.08 & 10.48 & 0.155 \\
\hline Type II & 0.88 & 11.8 & 0.18 \\
\hline Type III & 1.04 & 6.3 & 0.13 \\
\hline
\end{tabular}

The values of $\mathrm{n}(0.155,0.18$, and 0.13$)$ for different powder materials agree well with the range $(0.033 \leq n \leq 0.316)$ reported in the literature. The results of the 
experiments to measure $\mathrm{R}_{\text {pore }}$ and $\mathrm{a}_{\mathrm{p}}$ for each powder bed are also given in Table 7.4. With these values, $\mathrm{K}$ value could be calculated for each material system.

Table 7.4 Mean pore radius and pore density for different powder beds

\begin{tabular}{|c|c|c|}
\hline Powder grades & $\mathrm{R}_{\text {pore }}(\mu \mathrm{m})$ & $\mathrm{a}_{\mathrm{p}}(\mathbf{0})$ \\
\hline Type I & 0.9 & 49 \\
\hline Type II & 2.8 & 43 \\
\hline Type III & 4.9 & 39 \\
\hline
\end{tabular}

Having an equation for the droplet spreading radius as a function of time along with the K value for each powder bed, Eq. (7-3) can be used for determining the binder quantity penetrated the powder bed at any given time. As an instance, the equation below is used to calculate the binder volume penetrated the powder bed type I at any given time.

$$
V_{p}(t)=\frac{K}{2} \int_{0}^{t} \frac{\left(1.25(1.2+t)^{0.09}\right)^{2}}{\sqrt{t}} d t
$$

Therefore, at any given time, the amount of the liquid binder remaining on the powder bed surface can be calculated from Eq. (7-7). This amount should be substituted in one of the equations (7-8) or (7-9) depending on impact velocity to obtain equilibrium diameter for any given volume. As explained previously, with the droplet initial volume of $2 \mu \mathrm{l}$, and the impact velocity of $15 \mathrm{~mm} / \mathrm{s}$ the equation (7-9) would result in the higher value and therefore should be used in calculations for this study. As a result, the maximum diameter can be numerically found for any material system by specifying the point at which the equilibrium diameter and the diameter of the spreading droplet are equal. 
The equilibrium droplet radius for the volume of the liquid binder remaining on the powder bed surface predicted by Eq. (7-9) along with the spontaneous spreading droplet radius is plotted in Fig. 7.8 for different powder beds.

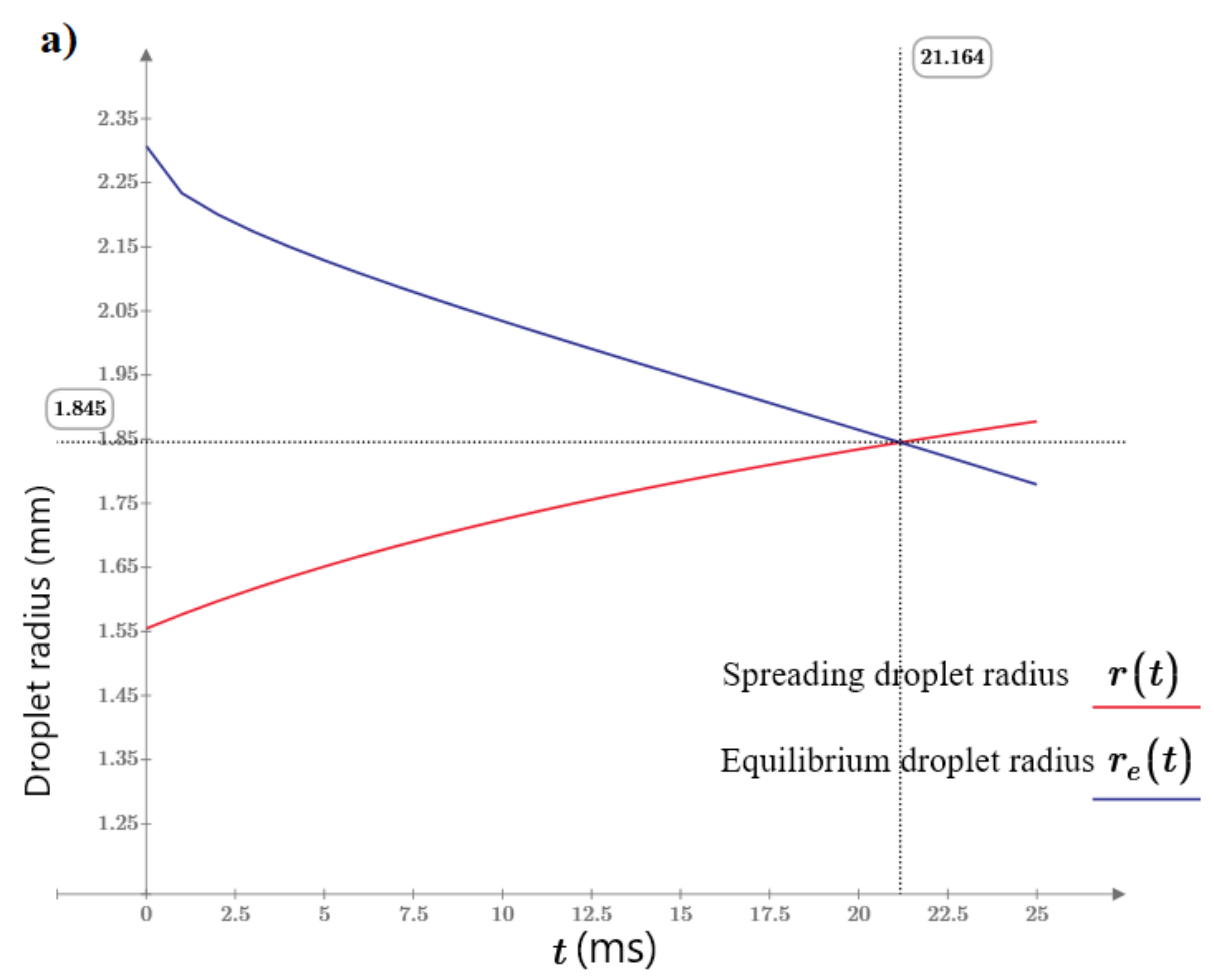



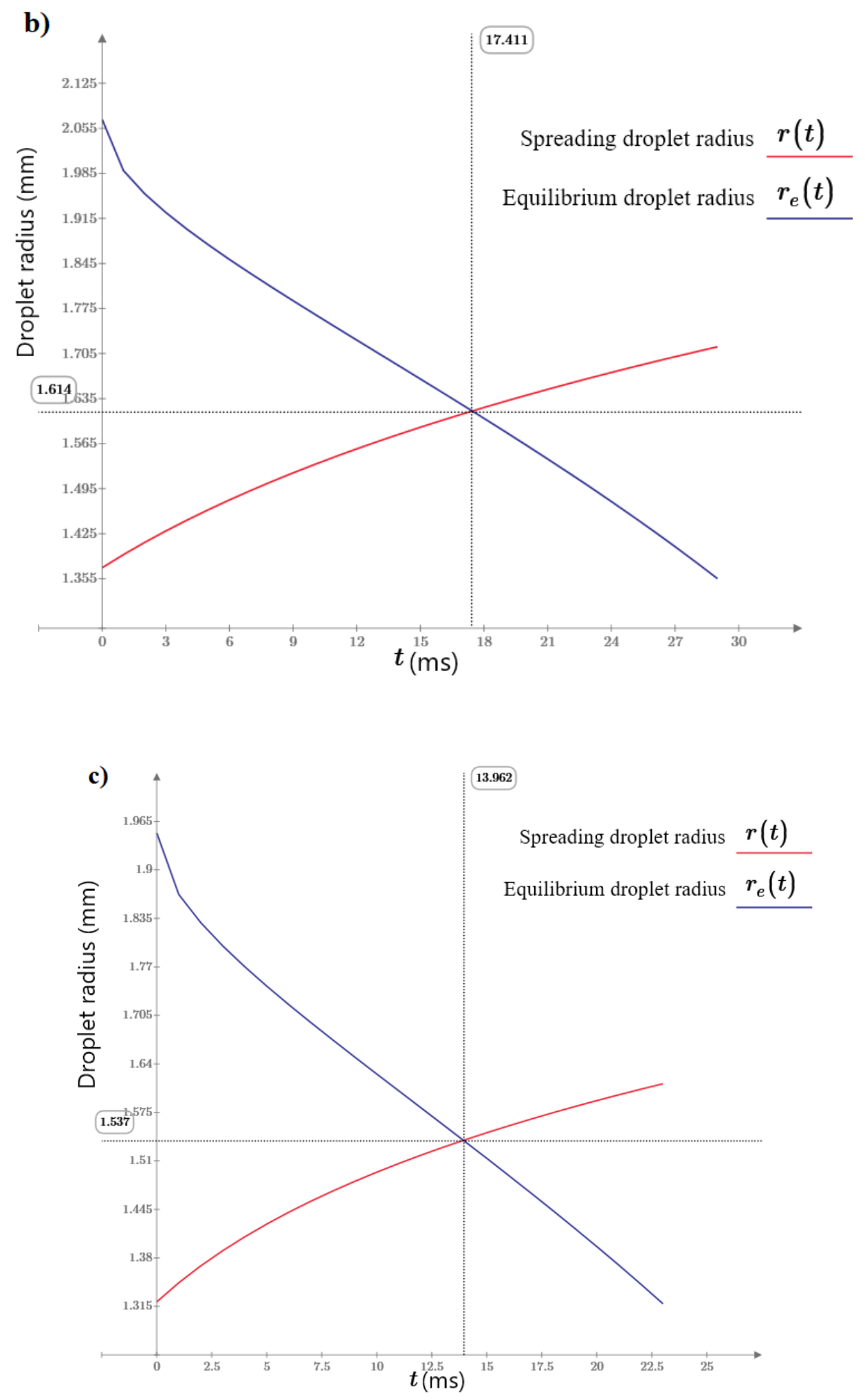

Fig. 7.8 Instantaneous spreading droplet radius (red color) and the equilibrium droplet radius (blue color) for powder beds a) type I b) type II c) type III 
From the developed droplet spreading model, the maximum spreading diameter (i.e. maximum wetted diameter after completer absorption of the droplet) are predicted to be 3.6, 3.2, and $2.92 \mathrm{~mm}$ for the powder beds of grade I, II, and III, respectively.

The results of the permeability measurements for different powder beds are reported in Table 7.5. Given the permeability values and the predicted maximum spreading radius, Eq. (7-11) cab be used to calculate the time it takes for a binder droplet of a certain initial volume to completely penetrate a powder bed of give particle size (pore size).

Table 7.5 The measured permeabilities for different powder materials

\begin{tabular}{|c|c|}
\hline Powder grades & $\mathrm{K}\left(\mathrm{cm}^{2}\right)$ \\
\hline Type I & $0.37 \times 10^{-9}$ \\
\hline Type II & $2.9 \times 10^{-9}$ \\
\hline Type III & $9.9 \times 10^{-9}$ \\
\hline
\end{tabular}

The time for the binder droplet of $2 \mu \mathrm{l}$ to fully infiltrate the powder beds of type I, II, III were determined to be 130,60 , and $50 \mathrm{~ms}$, respectively. Given these values for the droplet penetration time, Eq. (7-10) could be employed to determine the depth of penetration for each material system. The penetration depth and maximum spreading diameter for each powder material predicted using the developed model and the ones obtained experimentally are given in table below. 
Table 7.6 Predicted values for maximum spreading and penetration depth

\begin{tabular}{|c|c|c|c|c|c|c|}
\hline $\begin{array}{c}\text { Powder } \\
\text { grades }\end{array}$ & $\begin{array}{c}\mathrm{d}_{\text {max }} \text { from } \\
\text { the model } \\
(\mathrm{mm})\end{array}$ & $\begin{array}{c}\mathrm{d}_{\text {max }} \text { from } \\
\text { experiments } \\
(\mathrm{mm})\end{array}$ & $\begin{array}{c}\text { error for } \\
\mathrm{d}_{\max }(\%)\end{array}$ & $\begin{array}{c}\mathrm{h} \text { from the } \\
\text { model } \\
(\mathrm{mm})\end{array}$ & $\begin{array}{c}\mathrm{h} \text { from } \\
\text { experiments } \\
(\mathrm{mm})\end{array}$ & $\begin{array}{c}\text { error for } \\
\mathrm{h}(\%)\end{array}$ \\
\hline Type I & 3.6 & 3.9 & 8.3 & 0.57 & 0.51 & 11.8 \\
\hline Type II & 3.2 & 3.5 & 9.4 & 0.75 & 0.69 & 8.7 \\
\hline Type III & 3 & 3.2 & 6.7 & 0.91 & 0.85 & 7.1 \\
\hline
\end{tabular}

$\mathrm{d}_{\max }$ : Max spreading diameter

h: Penetration depth

Assuming a spherical cap for the area saturated by the droplet at equilibrium as schematically shown in Fig. 7.9, the predicted values for the droplet maximum spreading and penetration depth for any giving material system (i.e. a liquid binder and a power material) provide the necessary knowledge for estimating the droplet profile inside a powder material.

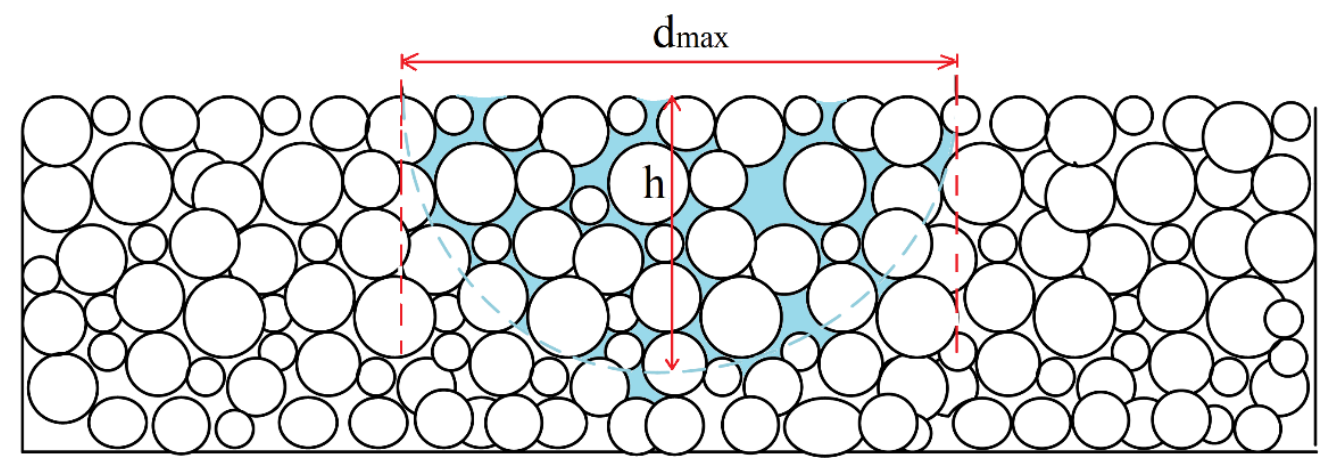

Fig. 7.9 schematic of a formed droplet profile inside the powder after complete penetration

From the results, it appears that the experimental results for the maximum spreading diameter are slightly higher than the ones predicted by the model. This could be partly attributed to the deviation of Park's model used for the prediction of the maximum spreading of a droplet on a solid surface $\left(\beta^{*}\right)$ in the impact driven phase. It has been reported in the literature the predications of this model falls in most cases within $10 \%$ of the 
experimental data [196]. Furthermore, the use of the mean pore size for calculating $\mathrm{K}$ in Eq. (7-6) could overestimate the droplet volume penetrated a powder bed at a given time, and as a result, lead to the predictions which underestimate the actual observed values for the maximum spreading. $\mathrm{K}$ which can be regards as a measure of permeability depends on pore size distribution in a way that the wide pore size distribution generally decreases the permeability below that calculated from the mean pore size $[95,206]$. The opposite trend is observed for the penetration depth predictions obtained from the model. In other words, the results predicted by the developed model for the penetration depth seem to have lower values than those obtained experimentally. This is attributable to the maximum spreading radius obtained from the model that is used in Eq. (7-11) for determining the droplet penetration time. As the model underestimates the maximum spreading radius, the use of this parameter results in longer estimated penetration time and consequently, higher penetration depth.

The results also reveal the significant influence of the particle size on the droplet/powder bed interaction and consequently, on the profile formed inside the powder bed after droplet complete penetration. The powder bed of type I (14 $\mu \mathrm{m}$ mean particle size) results in the largest diameter and the smallest penetration depth. In contrast, the powder material type III (with the mean particle size of $78 \mu \mathrm{m}$ ) leads to smallest diameter of the formed profile and the largest penetration depth. As shown in Fig. 7.10, while the depth of penetration increases with the mean particle size, the maximum diameter of the formed profiles shows decreasing trend when the mean particle increases. As explained in the previous chapter, such characteristics of binder/powder material interaction can be 
attributed to the change of the macroscopic surface roughness and pore morphologies with powder particle size distributions.

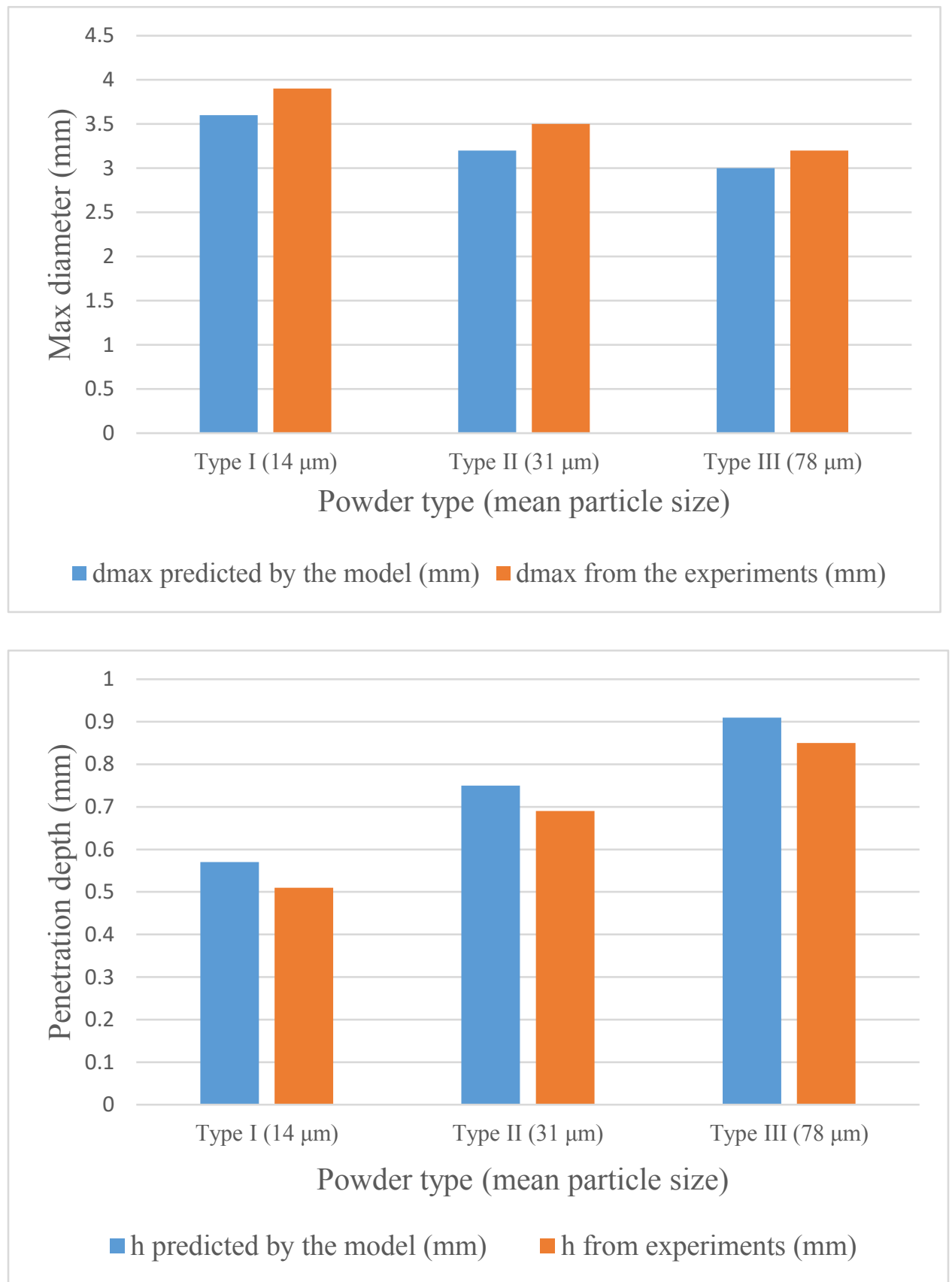

Fig. 7.10 Maximum diameter and depth of droplet profile inside the powder bed from the developed model and the experiments 
Fig. 7.11 shows the equilibrium droplet radius and the spontaneous spreading droplet radius for the different powder material types. From the figure, it appears that droplet spreading rate for all the powder types (I, II, and III) are approximately similar. In other words, despite the different initial spreading radius derived by the initial kinetic energy the rate of the droplet spreading radius in the capillary driven phase follow the same trend only shifted by a certain value. Interestingly, it is observed that at any given time the ratio of the instantaneous droplet radius for different powder material types are almost the same as the ratio of the $\beta^{*}$ (maximum spreading factor) for the corresponding powder materials. Therefore, given the rate of the droplet spreading radius on a powder material of a given particle size, it is possible to predict the spreading radius rate for the different particle sizes of the same powder material.

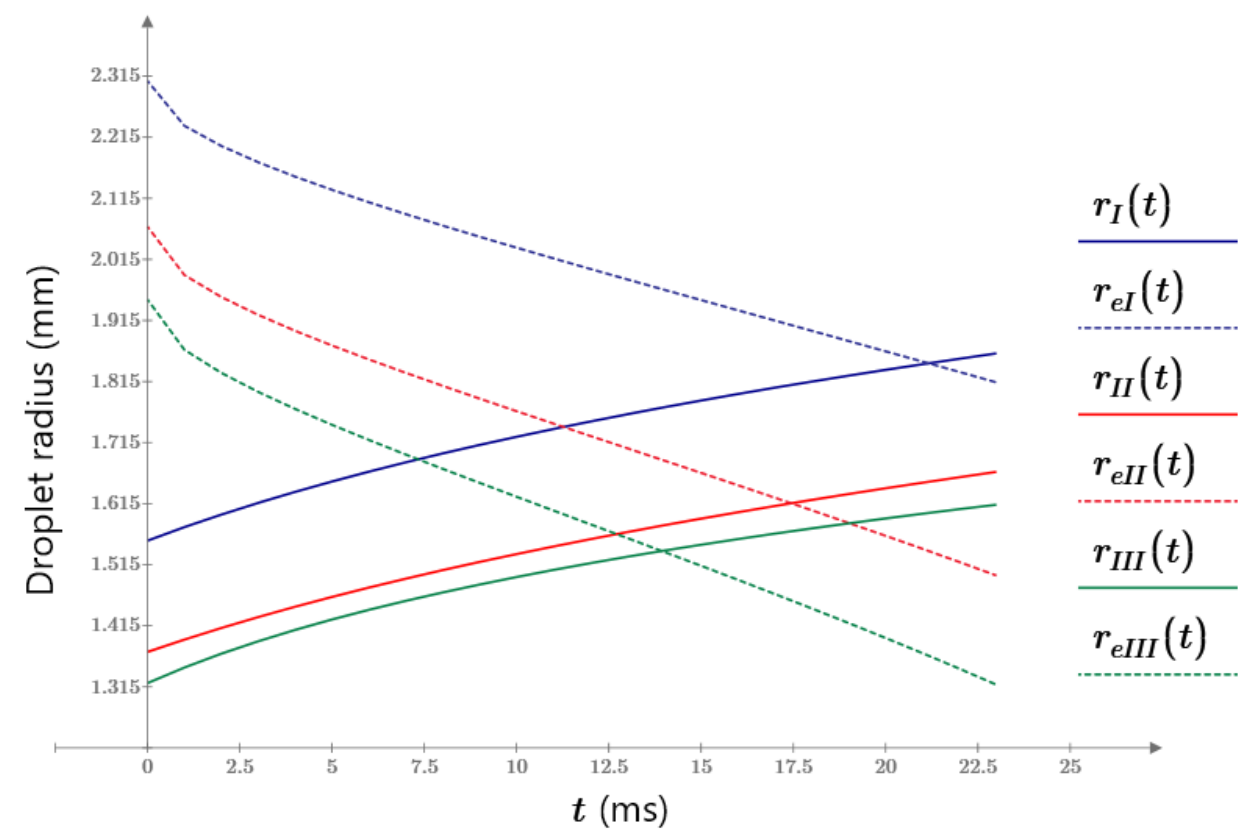

Fig. 7.11 Instantaneous spreading droplet radius and the equilibrium droplet radius for powder beds type I, II, and III 


\subsection{Conclusion}

A new model was developed based upon the droplet spreading/penetration dynamics for the prediction of a droplet profile inside a powder bed after complete penetration. The model was experimentally validated using different material systems. Good agreement was observed between the predictions of the developed model and the results from the experiments. The assumption of the spherical cap for the droplet profile inside a powder bed was also verified with the powder-binder interaction experiments. The results showed the significant influence of the powder particle size on the profile characteristics formed from the powder-droplet interaction. Powders with the larger mean particle size results in the larger profile diameter and the smaller penetration depth. The opposite trend is observed for the powder beds with the smaller particles. Despite the different initial spreading diameters in the capillary-driven phase, the powder beds with the different mean particle sizes show the same spreading rate in this phase. Therefore, the capillary spreading rate that is required as an input for the modeling can be experimentally obtained for powder bed of a certain particle size and applied for modeling of the different particle sizes. 


\section{CHAPTER 8}

\section{CONCLUSIONS}

In binder jetting additive manufacturing process, the main principle of the part fabrication is the application of a binding liquid agent in the selective areas of a powder bed through a drop-on-demand (DOD) printhead. The quality of the fabricated parts depends strongly upon the interaction of the liquid binder droplets with the powder particles. Under a static state (equilibrium condition), the interaction of the binder and powder bed determines the saturation amount required for optimal printing. In a dynamic state where the liquid binder wets the powder bed, such interaction determines the accuracy and the mechanical performance of the parts fabricated via binder jetting technology. The current research recognizes the crucial importance of the liquid binder-powder bed interaction and takes a deep look into the fundamentals of such interaction in an attempt to identify the key factors affecting the feature formation in binder jetting process. In this study, the interaction between the liquid binder and powder bed is systematically investigated, the effect of the different parameters is experimentally evaluated, and new physics-based models for the different phases of the interaction are developed in order to improve the part quality in binder jetting technology. 
For successful part fabrication in binder jetting process, an optimal amount of the liquid binder is required to ensure the structural integrity, the mechanical performance as well as the dimensional accuracy. Currently, selection of the optimal saturation level for any new material systems relies upon trial and error methods which in turn would impede the rapid development of new materials for this process. In this study, a new model based upon thermodynamics principles was developed to accurately predict the optimal saturation level with the aid of the experimental saturation-capillary pressure characterization curve. This model established basic insights on the correlation between the material characteristics and binder-powder interaction in the equilibrium state. It was found that characteristics such as binder surface tension, powder bed packing density, particle surface area, and powder material wettability strongly influences the binder penetration pattern and consequently the binder-powder material interaction. It was concluded that the primary force involved in the binder-powder interaction in the equilibrium state is the capillary force. The experimental data showed that the developed model results in more accurate predictions for powder particles with no microscopic surface areas. The existence of internal microscopic surface areas that do not contribute to the wetting of the powder would lead to overestimation of the optimal saturation levels as the internal surface areas were not accounted for in the model. The developed model is of critical importance in developing new material systems for the binder jetting technology, improving the accuracy and mechanical strength of printed parts, selecting optimal inprocess binder curing elements. 
In this dissertation, extensive experimental work was conducted to characterize the effect of different parameters on binder-powder material interaction and consequently on part quality. It was shown that printing speed could strongly affect the flow dynamics of the binder droplets impacting the powder bed surface. The quantitative correlation between the printing speed and part dimensional accuracy revealed the effect of binder droplet velocity on the binder-powder bed interaction. It was concluded that bouncing phenomenon of impinging droplets is less likely to occur in binder jetting technology due to the high wettability of the powder material to the liquid binder. The dependency of the dimensional accuracy of the fabricated features on the part orientation was attributed to the preferential spreading of the droplets due to the enhanced inertial forces in the printing direction. The results revealed that although the binder-powder material interaction in the dynamic phase was significantly influenced by the printing speed, in the equilibrium state the interaction did not seem to be affected by this parameter. From the results, it appeared that the droplet impact velocity significantly influences the powder-binder interaction and should be accounted for in the modeling of the interaction in the dynamic phase.

The real time imaging of the interaction of binder droplets with different powder materials using a high-speed camera revealed the strong correlation between the binder droplet flow dynamics and the powder material characteristics. It was observed that the flow dynamics of the binder droplets including spreading and penetration is significantly influenced by the powder particle sizes, which in turn affects the dimensional accuracies of the printed green parts. Powder beds consisted of larger particles facilitated the binder droplet vertical permeation. On the hand, binder lateral spreading over the surface increased for powder beds with smaller particle sizes. While the larger powder particle size 
helps with dimensional accuracies and green part densities of the BJ-AM parts, smaller powder particle size was beneficial to the surface finish of the fabricated parts, as well as higher mechanical properties and densities of the sintered parts. The gained insight about the relations between the different parameters and binder-powder interaction could be leveraged to select optimal process parameters, screen the materials for better precisions, and develop new models for various aspects of binder jetting technology.

After droplet complete penetration, the saturated area assumes a profile inside the powder bed. Such profile inside the powder bed as the smallest building element of any 3D printed component plays a key role in controlling the part quality and integrity. The characteristics of such profiles determines the lateral spacing between consecutive droplets and the optimum layer thickness for interlayer bonding and as results, influences the dimensional accuracy and the strength of the fabricated parts. In the present research, a predictive model was developed for droplet-powder interaction in the dynamic state to estimate the droplet profile characteristics inside the powder bed. It was assumed that the droplet profile after complete penetration into a power material is of a spherical cap. The developed model was to predict the diameter (i.e. maximum droplet spreading) and the height of such cap (the droplet penetration depth) in order to characterize the droplet profile inside any given powder material. The interaction of the binder droplet and powder bed at every stage of droplet evolution from the moment of impact to equilibrium state, where the interaction is complete, was carefully recorded and then analyzed. The entire interaction of the binder droplet and powder bed was divided into the impact-driven phase and capillarydriven phase. While in the impact-driven phase the main driving force for binder dynamics is the droplet impact velocity, in the capillary-driven phase the capillary forces dominate 
the interaction. Different formulations were considered for the analysis of each phase. The developed model was experimentally validated using different material systems. The results showed the significant influence of the powder particle size on the profile characteristics formed from the powder-droplet interaction. Powders with the larger mean particle size resulted in the larger profile diameter and the smaller penetration depth. The opposite trend was observed for the powder beds with the smaller particles. Despite the different initial spreading diameters in the capillary-driven phase, the powder beds with the different mean particle sizes showed the same spreading rate in this phase. Therefore, the capillary spreading rate that is required as an input for the modeling can be experimentally obtained for powder bed of a certain particle size and applied for modeling of the different particle sizes.

The models developed in this dissertation for the interaction of the liquid binderpowder material for both static and dynamic states could serve multiple purposes for advancing the binder jetting technology. The model for the binder-powder interaction in the equilibrium state can be employed to find out about the optimal saturation amount which is common printing parameter that is usually determined from experimental design for rapid development of new materials. The predictive model for droplet profile inside powder bed could be used to determine the optimal printing parameters such as binder droplet spacing and the layer thickness in binder jetting for any material system. Also, the correlations established from the modeling and the experimental work could be utilized for powder material and/or liquid binder screening for optimum dimensional accuracy and the mechanical strength. Furthermore, the developed models could be potentially used toward 
modeling the other aspects of binder jetting technology such as binder curing profile and binder burn-our profile during post-processing. 


\section{REFERENCES}

[1] Standard Terminology for Additive Manufacturing Technologies (F2792-12), http://www.astm.org, 2015.

[2] S.L.M. Ford, Additive Manufacturing Technology: Potential Implications for U.S. Manufacturing Competitiveness, Journal of International Commerce and Economics (2015) 1, 98571385.

[3] M. Derakhshani, T. Berfield, K.D. Murphy, Dynamic Analysis of a Bi-stable Buckled Structure for Vibration Energy Harvester, Springer International Publishing, Cham, 2018, pp. 199-208.

[4] B. Yao, F. Imani, A.S. Sakpal, E.W. Reutzel, H. Yang, Multifractal Analysis of Image Profiles for the Characterization and Detection of Defects in Additive Manufacturing, Journal of Manufacturing Science and Engineering 140(3) (2018) 031014-031014-13. [5] E.C. Santos, M. Shiomi, K. Osakada, T. Laoui, Rapid manufacturing of metal components by laser forming, International Journal of Machine Tools \& Manufacture (2006) 46, 1459-1468.

[6] M. Derakhshani, S. Hosseini-Hashemi, M. Fadaee, An analytical closed-form solution for free vibration of stepped circular/annular Mindlin functionally graded plate, 2018, p. 11.

[7] E.M. Sachs, J.S. Haggerty, M.J. Cima, P.A. Williams, Three-dimensional printing techniques, 1993.

[8] C. Bergmann, M. Lindner, W. Zhang, K. Koczur, A. Kirsten, R. Telle, H. Fischer, 3D printing of bone substitute implants using calcium phosphate and bioactive glasses, Journal of the European Ceramic Society 30(12) (2010) 2563-2567.

[9] T.M. Rankin, N.A. Giovinco, D.J. Cucher, G. Watts, B. Hurwitz, D.G. Armstrong, Three-dimensional printing surgical instruments: are we there yet?, Journal of Surgical Research 189(2) (2014) 193-197.

[10] A. Winkel, R. Meszaros, S. Reinsch, R. Müller, N. Travitzky, T. Fey, P. Greil, L. Wondraczek, Sintering of 3D-Printed Glass/HAp Composites, Journal of the American Ceramic Society 95(11) (2012) 3387-3393.

[11] A. Farzadi, M. Solati-Hashjin, M. Asadi-Eydivand, N.A. Abu Osman, Effect of Layer Thickness and Printing Orientation on Mechanical Properties and Dimensional Accuracy of 3D Printed Porous Samples for Bone Tissue Engineering, PLOS ONE 9(9) (2014) e108252.

[12] J. Moon, A.C. Caballero, L. Hozer, Y.-M. Chiang, M.J. Cima, Fabrication of functionally graded reaction infiltrated $\mathrm{SiC}-\mathrm{Si}$ composite by three-dimensional printing (3DPTM) process, Materials Science and Engineering: A 298(1-2) (2001) 110-119.

[13] H. Miyanaji, S. Zhang, A. Lassell, A.A. Zandinejad, L. Yang, Optimal Process Parameters for 3D Printing of Porcelain Structures, Procedia Manufacturing 5 (2016) $870-887$. 
[14] H. Miyanaji, S. Zhang, A. Lassell, A. Zandinejad, L. Yang, Process Development of Porcelain Ceramic Material with Binder Jetting Process for Dental Applications, JOM 68(3) (2016) 831-841.

[15] D. Snelling, H. Blount, C.A. Forman, K. Ramsburg, A. Wentzel, C.B. Williams, A. Druschitz, The Effects of 3d Printed Molds on Metal Castings, Proceedings of the Solid Freeform Fabrication Symposium, Austin, TX, 2014.

[16] https://corporate.ford.com/innovation/building-in-the-automotive-sandbox.html.

[17] Y. Bai, C.B. Williams, An exploration of binder jetting of copper, Rapid Prototyping Journal (2015) 177 - 185.

[18] D. Günther, F. Mögele, Additive Manufacturing of Casting Tools Using PowderBinder- Jetting Technology, New Trends in 3D Printing, InTech2016, p. 268.

[19] A. Della Bona, P.H. Corazza, Y. Zhang, Characterization of a polymer-infiltrated ceramic-network material, Dental materials : official publication of the Academy of Dental Materials 30(5) (2014) 564-9.

[20] I. Gibson, D. Rosen, B. Stucker, Additive manufacturing technologies : 3D printing, rapid prototyping, and direct digital manufacturing, Springer, New York, NY :, 2015. [21] Steve Upcraft, R. Fletcher, The rapid prototyping technologies, Assembly Automation 23(4) (2003) 318-330.

[22] N. Ramakrishnan, P.K. Rajesh, P. Ponnambalam, K. Prakasan, Studies on preparation of ceramic inks and simulation of drop formation and spread in direct ceramic inkjet printing, Journal of Materials Processing Technology 169(3) (2005) 372381.

[23] L. Yang, S. Zhang, G. Oliveira, B. Stucker, Development of a 3D Printing Method for Production of Dental Application, Proceedings of the 24th International Solid Freeform Fabrication Symposium, Austin, TX, USA, 2013.

[24] J.F. Bredt, Binder Stability and Powder \Binder Interaction in Three Dimensional

Printing, Dept. of Mechanical Engineering, Massachusetts Institute of Technology, Cambridge, 1995, p. 382

[25] W.T.R. Kathy Lu, 3DP process for fine mesh structure printing, (2008).

[26] M. Vaezi, C.K. Chua, Effects of layer thickness and binder saturation level

parameters on 3D printing process, The International Journal of Advanced Manufacturing Technology 53(1) (2011) 275-284.

[27] T.D. Szucs, D. Brabazon, Effect of Saturation and Post Processing on 3D Printed Calcium Phosphate Scaffolds, Key Engineering Materials 396-398 (2009) 663-666. [28] M. Asadi-Eydivand, M. Solati-Hashjin, A. Farzad, N.A. Abu Osman, Effect of technical parameters on porous structure and strength of 3D printed calcium sulfate prototypes, Robotics and Computer-Integrated Manufacturing 37 (2016) 57-67.

[29] J. Suwanprateeb, F. Thammarakcharoen, K. Wasoontararat, W. Suvannapruk, Influence of printing parameters on the transformation efficiency of 3D-printed plaster of paris to hydroxyapatite and its properties, Rapid Prototyping Journal 18(6) (2012) 490499.

[30] P. Feng, X. Meng, J.-F. Chen, L. Ye, Mechanical properties of structures 3D printed with cementitious powders, Construction and Building Materials 93 (2015) 486-497. [31] F. Fremond, S. Miyazaki, Shape Memory Alloys, Springer Wien, New York, 1996. [32] A. Butscher, M. Bohner, C. Roth, A. Ernstberger, R. Heuberger, N. Doebelin, P. Rudolf von Rohr, R. Rudolf Printability of calcium phosphate powders for three- 
dimensional printing of tissue engineering scaffolds, Acta Biomaterialia 8(1) (2012) 373385.

[33] E. Teunou, J.J. Fitzpatrick, E.C. Synnott, Characterisation of food powder flowability, Journal of Food Engineering 39(1) (1999) 31-37.

[34] A. Balakrishnan, P. Pizette, C.L. Martin, Effect of particle size in aggregated and agglomerated ceramic powders, Acta Materialia 58 (2010) 802-812.

[35] I. MacLaren, P.A. Trusty, C.B. Ponton, A transmission electron microscope study of hydrothermally synthesized yttrium disilicate powders, Acta Materialia 47 (1999) 779791.

[36] J.C. Sánchez-López, A. Fernández, TEM study of fractal scaling in nanoparticle agglomerates obtained by gas-phase condensation, Acta Materialia 48 (2000) 3761-3771. [37] J.G. Li, T. Ikegami, J.H. Lee, T. Mori, Characterization and sintering of nanocrystalline $\mathrm{CeO} 2$ powders synthesized by a mimic alkoxide method, Acta Materialia 49 (2001) 419-426.

[38] R.M. German, Powder metallurgy science, Metal Powder Industries Federation, Princeton, N.J. :, 1984.

[39] M.J. O'Hara, I.B. Cutler, Sintering Kinetics of binary mixtures of alumina powders, Proceedings of the British Ceramic Society, 1969, pp. 145-154.

[40] E.S. J.M. Benson, THE NEED FOR POWDER CHARACTERISATION IN THE ADDITIVE MANUFACTURING INDUSTRY AND THE ESTABLISHMENT OF A NATIONAL FACILITY, South African Journal of Industrial Engineering 26(2) (2015) 104-114.

[41] C.C. Furnas, M. United States. Bureau of, The relations between specific volume, voids, and size composition in systems of broken solids of mixed sizes, Dept. of Commerce, Bureau of Mines, Washington, D.C. :, 1928.

[42] A.E.R. Westman, H.R. Hugill, THE PACKING OF PARTICLES1, Journal of the American Ceramic Society 13(10) (1930) 767-779.

[43] A.H.M. Andreasen, J. Andersen, Relation between grain size and interstitial space in products of unconsolidated granules, Kolloid-Z 50 (1930) 271-281.

[44] J.R. Schuhmann, Principles of comminution: size distribution and surface calculation, The American Institute of Mining, Metallurgical, and Petroleum Engineers

1940, p. 11.

[45] R.K. McGeary, Mechanical Packing of Spherical Particles, Journal of the American Ceramic Society 44(10) (1961) 513-522.

[46] H.Y. Sohn, C. Moreland, The effect of particle size distribution on packing density, The Canadian Journal of Chemical Engineering 46(3) (1968) 162-167.

[47] A. Streek, P. Regenfuss, R. Ebert, H. Exner, Laser micro sintering -a quality leap through improvement of powder packing, Proceedings of the 19th International Solid Freeform Fabrication Symposium, Austin, Texas, USA, 2008.

[48] G. Egger, P.E. Gygax, R. Glardon, N.P. Karapatis, Optimization of powder layer density in selective laser sintering, University Texas Austin, Mechanical Engineering Dept, Austin, Tx 00000 Usa, pp. 255-263-255-263.

[49] M. Alonso, M. Satoh, K. Miyanami, Void-size distribution in two-dimensional random packings of equal-sized disks, The Canadian Journal of Chemical Engineering 70(1) (1992) 28-32. 
[50] R.P. Koseski, P. Suri, N.B. Earhardt, R.M. German, Y.-S. Kwon, Microstructural evolution of injection molded gas- and water-atomized 316L stainless steel powder during sintering, Materials Science and Engineering: A 390(1) (2005) 171-177.

[51] C. Vincent, J.F. Silvain, J.M. Heintz, N. Chandra, Effect of porosity on the thermal conductivity of copper processed by powder metallurgy, Journal of Physics and Chemistry of Solids 73(3) (2012) 499-504.

[52] E.W. Washburn, The Dynamics of Capillary Flow, Physical Review 17(3) (1921) 273-283.

[53] M. Denesuk, G.L. Smith, B.J.J. Zelinski, N.J. Kreidl, D.R. Uhlmann, Capillary Penetration of Liquid Droplets into Porous Materials, Journal of Colloid and Interface Science Volume 158(Issue 1) (1992) Pages 114-120.

[54] M. Denesuk, B.J.J. Zelinski, N.J. Kreidl, D.R. Uhlmann, Dynamics of Incomplete Wetting on Porous Materials, Journal of Colloid and Interface Science 168(1) (1994) 142-151.

[55] H. Miyanaji, S. Zhang, L. Yang, A new physics-based model for equilibrium saturation determination in binder jetting additive manufacturing process, International Journal of Machine Tools and Manufacture 124(Supplement C) (2018) 1-11. [56] A. MARMUR, Drop Penetration into a Thin Porous Medium, (1987).

[57] A. Marmur, Penetration of a Small Drop into a Capillary, Journal of Colloid and Interface Science 122(1) (1988) 209-219.

[58] S.M. Gaytan, M.A. Cadena, H. Karim, D. Delfin, Y. Lin, D. Espalin, E. MacDonald, R.B. Wicker, Fabrication of barium titanate by binder jetting additive manufacturing technology, Ceramics International 41(5, Part A) (2015) 6610-6619.

[59] J.H. Weaver, The world of physics: a small library of the literature of physics from antiquity to the present, Simon \& Schuster, New York, 1987, pp. 579-582.

[60] J. Bear, Dynamics of fluids in porous media, Elsevier, New York, 1972.

[61] K.P. Hapgood, J.D. Litster, S.R. Biggs, T. Howes, Drop Penetration into Porous Powder Beds, Journal of Colloid and Interface Science 253(2) (2002) 353-366.

[62] Y.Y.a.T.R. Lee, Contact Angle and Wetting Properties.

[63] S. Middleman, Modeling flows in films, jets, and drops, Chemical Engineering Education 29 (1995) 210-217.

[64] R. Lucas, Ueber das Zeitgesetz des Kapillaren Aufstiegs von Flussigkeiten, Kolloid Z. 23(1) (1918) 15-22.

[65] E.K. Rideal, On the flow of liquids under capillary pressure, Philos. Mag. Ser. 264 (1922) 1152-1159.

[66] C.H. Bosanquet, On the flow of liquid into capillary tubes, Philos. Mag. Ser. 45(6) (1923) 525-531.

[67] J. Szekely, A.W. Neumann, Y.K. Chuang, The rate of capillary penetration and the applicability of the washburn equation, Journal of Colloid and Interface Science 35(2) (1971) 273-278.

[68] S. Levine, P. Reed, E.J. Watson, G. Neal, A theory of the rate of rise of a liquid in a capillary, Journal of Colloid and Interface Science 3 (1976) 403.

[69] M.F. Letelier, H.J. Leutheusser, Approximate analysis of unsteady laminar flow, J. Colloid Interface Sci 107 (1981) 1278-1280.

[70] G.L. Batten, Liquid imbibition in capillaries and packed beds, Journal of Colloid and Interface Science 84 (1984) 513-518. 
[71] R.J. Good, Transport of a fluid in a porous medium: a generalization of the Washburn equation, Chem. Ind. 22 (1971) 600-613.

[72] R.J. Hansen, T.Y. Toong, Dynamic contact angle and its relationship to forces of hydrodynamic origin, J. Colloid Interface Sci 37 (1971) 196-207.

[73] C. Huh, S.G. Mason, Effects of surface roughness on wetting (theoretical), Journal of Colloid and Interface Science 35 (1977) 568-581.

[74] C. Huh, L.E. Striven, Hydrodynamic model of steady movement of a solid/liquid/fluid contact line, Journal of Colloid and Interface Science 35 (1971) 85-101. [75] J.V. Brakel, P.M. Heertjes, Capillary rise in porous media, Powder Technology 16(1) (1977) 75-81.

[76] S. Levine, J. Lowndes, E.J. Watson, Neale, A theory of capillary rise of a liquid in a vertical cylindrical tube and in a parallel-plate channel, J. Colloid Interf. Sci. 73 (1980) 136-151.

[77] R.A. Martynov, V.V. Malev, E.V. Gribanova, Kinetics of capillary rise, Colloid J. USSR (USA) 3 (1983) 245-250.

[78] L. Grader, On the modelling of the dynamic contact angle, Colloid and Polymer Science 264(8) (1986) 719-726.

[79] F.A.L. Dullien, M.S. EI-Sayed, V.K. Batra, Rate of capillary rise in porous media, J. Colloid Interface Sci. 60 (1977) 497.

[80] S. Levine, G. Neal, Theory of the rate of wetting of a porous medium, J. Chem. Soc., Faraday Trans 71(2) (1975) 12-21.

[81] S. Levine, P. Lowndes, J. Reed, Two phase fluid flow and hysteresis in a periodic capillary tube, J. Colloid Interf. Sci. 77 (1980) 253-263.

[82] T.E. Mumley, C.J. Radke, M.C. Williams, Kinetics of liquid/liquid capillary rise.

1.Experimental observations, J. Colloid Interf. Sci. 109(2) (1986) 398-412.

[83] A. MARMUR, The Radial Capillary, (1987).

[84] The radial capillary, Journal of Colloid and Interface Science 124(1) (1988) 301308.

[85] Capillary Penetration of Liquid Droplets into Porous Materials, Journal of Colloid and Interface Science 158 (1992) 114-120.

[86] A. Clarke, T.D. Blake, K. Carruthers, A. Woodward, Spreading and Imbibition of Liquid Droplets on Porous Surfaces, Langmuir 18(8) (2002) 2980-2984.

[87] T.D. Blake, A. Clarke, J. De Coninck, M.J. de Ruijter, Contact Angle Relaxation during Droplet Spreading: Comparison between Molecular Kinetic Theory and Molecular Dynamics, Langmuir 13(7) (1997) 2164-2166.

[88] T.D. Blake, The physics of moving wetting lines, J Colloid Interface Sci 299(1) (2006) 1-13.

[89] V.M. Starov, S.R. Kostvintsev, V.D. Sobolev, M.G. Velarde, S.A. Zhdanov, Spreading of Liquid Drops over Dry Porous Layers: Complete Wetting Case, Journal of Colloid and Interface Science 252(2) (2002) 397-408.

[90] V.M. Starov, S.A. Zhdanov, S.R. Kosvintsev, V.D. Sobolev, M.G. Velarde, Spreading of liquid drops over porous substrates, Advances in Colloid and Interface Science 104(1-3) (2003) 123-158.

[91] H. Tan, Absorption of picoliter droplets by thin porous substrates, AIChE Journal (2016) n/a-n/a. 
[92] R.C. Daniel, J.C. Berg, Spreading on and penetration into thin, permeable print media: Application to ink-jet printing, Advances in Colloid and Interface Science 123126 (2006) 439-469.

[93] V.M. Starov, S.A. Zhdanov, M.G. Velarde, Spreading of Liquid Drops over Thick Porous Layers: Complete Wetting Case, Langmuir 18(25) (2002) 9744-9750.

[94] K.L. Yip, A.R. Lubinsky, D.R. Perchak, K.C. Ng, Measurement and Modeling of Drop Absorption Time for Various Ink-receiver Systems, Journal of Imaging Science and Technology 47(5) (2003) 388-393.

[95] R.K. Holman, M.J. Cima, S.A. Uhland, E. Sachs, Spreading and Infiltration of Inkjet-Printed Polymer Solution Droplets on a Porous Substrate, Journal of Colloid and Interface Science 249(2) (2002) 432-440.

[96] T. Fan, droplet-powder impact inteaction in three dimensional printing, Department of Mechanical Engineering, MIT, 1995.

[97] P. Nandwana, A.M. Elliott, D. Siddel, A. Merriman, W.H. Peter, S.S. Babu, Powder bed binder jet 3D printing of Inconel 718: Densification, microstructural evolution and challenges $\aleph^{2}$, Current Opinion in Solid State and Materials Science 21(4) (2017) 207218.

[98] Y. Bai, C.B. Williams, An exploration of binder jetting of copper, Rapid Prototyping Journal 21(2) (2015) 177-185.

[99] J. Yoo, M. Cima, E. Sachs, S. Suresh, Fabrication and Microstructural Control of Advanced Ceramic Components by Three Dimensional Printing, Proceedings of the 19th Annual Conference on Composites, Advanced Ceramics, Materials, and Structures-B: Ceramic Engineering and Science Proceedings, John Wiley \& Sons, Inc.2008, pp. 755762.

[100] E. Sachs, M. Cima, J. Cornie, Three-Dimensional Printing: Rapid Tooling and Prototypes Directly from a CAD Model, CIRP Annals - Manufacturing Technology 39(1) (1990) 201-204.

[101] A.W. Adamson, A.P. Gast, Capillarity, Physical Chemistry of Surfaces, Wiley, New York, 1991, pp. 4-49.

[102] A. Marmur, Kinetics of Penetration into Uniform Porous Media: Testing the Equivalent-Capillary Concept, Langmuir 19(14) (2003) 5956-5959.

[103] H. Miyanaji, L. Yang, Equilibrium Saturation In Binder Jetting Additive Manufacturing Process: Theoretical Model Vs. Experimental Observations, 2016 Annual International Solid Freeform Fabrication Symposium (SFF Symp 2016), Austin, TX,USA, 2016.

[104] J.F. Bredt, Binder Stability and Powder|Binder Interaction in Three Dimensional Printing, Dept. of Mechanical Engineering, Massachusetts Institute of Technology, Cambridge, 1995, p. 382.

[105] K. Lu, W.T. Reynolds, 3DP process for fine mesh structure printing, Powder Technology 187(1) (2008) 11-18.

[106] F.M. Orr, J.J. Taber, Use of Carbon Dioxide in Enhanced Oil Recovery, Science 224(4649) (1984) 563-569.

[107] L. Cueto-Felgueroso, R. Juanes, Nonlocal Interface Dynamics and Pattern Formation in Gravity-Driven Unsaturated Flow through Porous Media, Physical Review Letters 101(24) (2008) 244504. 
[108] L. Paterson, Diffusion-Limited Aggregation and Two-Fluid Displacements in Porous Media, Physical Review Letters 52(18) (1984) 1621-1624.

[109] G. Løvoll, Y. Méheust, K.J. Måløy, E. Aker, J. Schmittbuhl, Competition of gravity, capillary and viscous forces during drainage in a two-dimensional porous medium, a pore scale study, Energy 30(6) (2005) 861-872.

[110] B. Zhao, C.W. MacMinn, R. Juanes, Wettability control on multiphase flow in patterned microfluidics, Proceedings of the National Academy of Sciences 113(37) (2016) 10251-10256.

[111] S.H. Davis, L.M. Hocking, Spreading and imbibition of viscous liquid on a porous base, Physics of Fluids 11(1) (1999) 48-57.

[112] K. Range, F. Feuillebois, Influence of Surface Roughness on Liquid Drop Impact, Journal of Colloid and Interface Science 203(1) (1998) 16-30.

[113] J.B. Lee, A.I. Radu, P. Vontobel, D. Derome, J. Carmeliet, Absorption of impinging water droplet in porous stones, Journal of Colloid and Interface Science 471 (2016) 59-70.

[114] R. Lenormand, Liquids in porous media, Journal of Physics: Condensed Matter 2(S) (1990) SA79.

[115] R. Lenormand, E. Touboul, C. Zarcone, Numerical models and experiments on immiscible displacements in porous media, Journal of Fluid Mechanics 189 (1988) 165187.

[116] R. Holtzman, Effects of Pore-Scale Disorder on Fluid Displacement in PartiallyWettable Porous Media, Scientific Reports 6 (2016) 36221.

[117] P. Concus, R. Finn, ON THE BEHAVIOR OF A CAPILLARY SURFACE IN A WEDGE, Proceedings of the National Academy of Sciences 63(2) (1969) 292-299. [118] M. Cieplak, M.O. Robbins, Dynamical Transition in Quasistatic Fluid Invasion in Porous Media, Physical Review Letters 60(20) (1988) 2042-2045.

[119] M. Cieplak, M.O. Robbins, Influence of contact angle on quasistatic fluid invasion of porous media, Physical Review B 41(16) (1990) 11508-11521.

[120] N. Martys, M. Cieplak, M.O. Robbins, Critical phenomena in fluid invasion of porous media, Physical Review Letters 66(8) (1991) 1058-1061.

[121] S.W. Rienstra, The shape of a sessile drop for small and large surface tension, Journal of Engineering Mathematics 24(3) (1990) 193-202.

[122] E. Nowak, G. Combes, E.H. Stitt, A.W. Pacek, A comparison of contact angle measurement techniques applied to highly porous catalyst supports, Powder Technology 233 (2013) 52-64.

[123] J. Shang, M. Flury, J.B. Harsh, R.L. Zollars, Comparison of different methods to measure contact angles of soil colloids, Journal of Colloid and Interface Science 328(2) (2008) 299-307.

[124] S.M. Kumar, A.P. Deshpande, Dynamics of drop spreading on fibrous porous media, Colloids and Surfaces A: Physicochemical and Engineering Aspects 277(1-3) (2006) 157-163.

[125] T.C. Chao, O. Arjmandi-Tash, D.B. Das, V.M. Starov, Spreading of blood drops over dry porous substrate: Complete wetting case, Journal of Colloid and Interface Science 446 (2015) 218-225.

[126] D.G. Fredlund, H. Rahardjo, Measurements of Soil Suction, Soil Mechanics for Unsaturated Soils, Wiley, New York, 1993, pp. 64-106. 
[127] M. Tuller, D. Or, WATER RETENTION AND CHARACTERISTIC CURVE A2 Hillel, Daniel, Encyclopedia of Soils in the Environment, Elsevier, Oxford, 2005, pp. 278-289.

[128] R. Chumnanklang, T. Panyathanmaporn, K. Sitthiseripratip, J. Suwanprateeb, 3D printing of hydroxyapatite: Effect of binder concentration in pre-coated particle on part strength, Materials Science and Engineering: C 27(4) (2007) 914-921.

[129] S. Shrestha, G. Manogharan, Optimization of Binder Jetting Using Taguchi Method, JOM 69(3) (2017) 491-497.

[130] M.A. Caradonna, M.J. Cima, J. Grau, J. Moon, E.M. Sachs, P.C. Saxton, J.G. Serdy, S.A. Uhland, Jetting layers of powder and the formation of fine powder beds thereby, Google Patents, 2000.

[131] J. Trapp, A.M. Rubenchik, G. Guss, M.J. Matthews, In situ absorptivity measurements of metallic powders during laser powder-bed fusion additive manufacturing, Applied Materials Today 9(Supplement C) (2017) 341-349. [132] P. Bidare, I. Bitharas, R.M. Ward, M.M. Attallah, A.J. Moore, Fluid and particle dynamics in laser powder bed fusion, Acta Materialia 142(Supplement C) (2018) 107120.

[133] I. Gibson, D. Rosen, B. Stucker, Vat Photopolymerization Processes, Additive Manufacturing Technologies: 3D Printing, Rapid Prototyping, and Direct Digital Manufacturing, Springer New York, New York, NY, 2015, pp. 63-106. [134] D.W. Rosen, Stereolithography and Rapid Prototyping, in: P.J. Hesketh (Ed.), BioNanoFluidic MEMS, Springer US, Boston, MA, 2008, pp. 175-196.

[135] M. Duan, D. Yoon, C.E. Okwudire, A limited-preview filtered B-spline approach to tracking control - With application to vibration-induced error compensation of a 3D printer, Mechatronics (2017).

[136] A.a. Alafaghani, A. Qattawi, B. Alrawi, A. Guzman, Experimental Optimization of Fused Deposition Modelling Processing Parameters: A Design-for-Manufacturing Approach, Procedia Manufacturing 10(Supplement C) (2017) 791-803.

[137] P. Alam, M. Toivakka, K. Backfolk, P. Sirviö, Impact spreading and absorption of Newtonian droplets on topographically irregular porous materials, Chemical Engineering Science 62(12) (2007) 3142-3158. [138] J.O. Marston, S.T. Thoroddsen, W.K. Ng, R.B.H. Tan, Experimental study of liquid drop impact onto a powder surface, Powder Technology 203(2) (2010) 223-236. [139] Z. Yu, F. Wang, L.S. Fan, Experimental and Numerical Studies of Water Droplet Impact on a Porous Surface in the Film-Boiling Regime, Industrial \& Engineering Chemistry Research 47(23) (2008) 9174-9182.

[140] S. Chandra, C.T. Avedisian, Observations of droplet impingement on a ceramic porous surface, International Journal of Heat and Mass Transfer 35(10) (1992) 23772388.

[141] A.L. Yarin, DROP IMPACT DYNAMICS: Splashing, Spreading, Receding, Bouncing..., Annual Review of Fluid Mechanics 38(1) (2006) 159-192.

[142] D.G.K. Aboud, A.-M. Kietzig, Splashing Threshold of Oblique Droplet Impacts on Surfaces of Various Wettability, Langmuir 31(36) (2015) 10100-10111.

[143] P. Attané, F. Girard, V. Morin, An energy balance approach of the dynamics of drop impact on a solid surface, Physics of Fluids 19(1) (2007) 012101. 
[144] R. Martin, Phenomena of liquid drop impact on solid and liquid surfaces, Fluid Dynamics Research 12(2) (1993) 61.

[145] Š. Šikalo, M. Marengo, C. Tropea, E.N. Ganić, Analysis of impact of droplets on horizontal surfaces, Experimental Thermal and Fluid Science 25(7) (2002) 503-510. [146] R. Rioboo, M. Marengo, C. Tropea, Time evolution of liquid drop impact onto solid, dry surfaces, Experiments in Fluids 33(1) (2002) 112-124.

[147] T. Mao, D.C.S. Kuhn, H. Tran, Spread and rebound of liquid droplets upon impact on flat surfaces, AIChE Journal 43(9) (1997) 2169-2179.

[148] D. Bartolo, F. Bouamrirene, V. É, A. Buguin, P. Silberzan, S. Moulinet, Bouncing or sticky droplets: Impalement transitions on superhydrophobic micropatterned surfaces, EPL (Europhysics Letters) 74(2) (2006) 299.

[149] M. Reyssat, A. Pépin, F. Marty, Y. Chen, D. Quéré, Bouncing transitions on microtextured materials, EPL (Europhysics Letters) 74(2) (2006) 306.

[150] D. Richard, C. Clanet, D. Quere, Surface phenomena: Contact time of a bouncing drop, Nature 417(6891) (2002) 811-811.

[151] C. Clanet, C. BÉGuin, D. Richard, D. QuÉRÉ, Maximal deformation of an impacting drop, Journal of Fluid Mechanics 517 (2004) 199-208.

[152] C. Antonini, F. Villa, I. Bernagozzi, A. Amirfazli, M. Marengo, Drop Rebound

after Impact: The Role of the Receding Contact Angle, Langmuir 29(52) (2013) 16045 16050.

[153] R. Rioboo, M. Voué, A. Vaillant, J. De Coninck, Drop Impact on Porous Superhydrophobic Polymer Surfaces, Langmuir 24(24) (2008) 14074-14077.

[154] B.L. Scheller, D.W. Bousfield, Newtonian drop impact with a solid surface, AIChE Journal 41(6) (1995) 1357-1367.

[155] B. Utela, D. Storti, R. Anderson, M. Ganter, A review of process development steps for new material systems in three dimensional printing (3DP), Journal of Manufacturing Processes 10(2) (2008) 96-104.

[156] S. Maleksaeedi, H. Eng, F.E. Wiria, T.M.H. Ha, Z. He, Property enhancement of 3D-printed alumina ceramics using vacuum infiltration, Journal of Materials Processing Technology 214(7) (2014) 1301-1306.

[157] Y. Bai, G. Wagner, C.B. Williams, Effect of Particle Size Distribution on Powder Packing and Sintering in Binder Jetting Additive Manufacturing of Metals, Journal of Manufacturing Science and Engineering 139(8) (2017) 081019-081019-6.

[158] B. Derby, Inkjet Printing of Functional and Structural Materials: Fluid Property Requirements, Feature Stability, and Resolution, ANNUAL REVIEW OF MATERIALS RESEARCH 40 (2010) 395-414.

[159] J. Moon, J.E. Grau, V. Knezevic, M.J. Cima, E.M. Sachs, Ink-Jet Printing of

Binders for Ceramic Components, Journal of the American Ceramic Society 85(4) (2002) 755-762.

[160] K. .Picha, C. Spackman, J. Samuel, Droplet spreading characteristics observed during 3D printing of aligned fiber-reinforced soft composites, Additive Manufacturing 12, Part A (2016) 121-131.

[161] T. Lim, S. Han, J. Chung, J.T. Chung, S. Ko, C.P. Grigoropoulos, Experimental study on spreading and evaporation of inkjet printed pico-liter droplet on a heated substrate, International Journal of Heat and Mass Transfer 52(1-2) (2009) 431-441. 
[162] J. Zheng, W.B. Carlson, J.S. Reed, The packing density of binary powder mixtures, Journal of the European Ceramic Society 15(5) (1995) 479-483.

[163] A.B. Spierings, N. Herres, G. Levy, Influence of the particle size distribution on surface quality and mechanical properties in AM steel parts, Rapid Prototyping Journal 17(3) (2011) 195-202.

[164] A. Townsend, N. Senin, L. Blunt, R.K. Leach, J.S. Taylor, Surface texture metrology for metal additive manufacturing: a review, Precision Engineering 46 (2016) 34-47.

[165] A.T. Sutton, C.S. Kriewall, M.C. Leu, J.W. Newkirk, Powder characterisation techniques and effects of powder characteristics on part properties in powder-bed fusion processes, Virtual and Physical Prototyping 12(1) (2017) 3-29.

[166] H. Miyanaji, N. Momenzadeh, L. Yang, Effect of printing speed on quality of printed parts in Binder Jetting Process, Additive Manufacturing 20 (2018) 1-10.

[167] A.M. Elliott, P. Nandwana, D.H. Siddel, B. Compton, A Method for Measuring Powder Bed Density in Binder Jet Additive Manufacturing Process and the Powder Feedstock Characteristics Influencing the Powder Bed Density, Oak Ridge National Laboratory (ORNL), Oak Ridge, TN (United States). High Temperature Materials Laboratory (HTML); Manufacturing Demonstration Facility (MDF), 2016.

[168] K.W. Desmond, E.R. Weeks, Influence of particle size distribution on random close packing of spheres, Physical Review E 90(2) (2014) 022204.

[169] H.J.H. Brouwers, Particle-size distribution and packing fraction of geometric random packings, Physical Review E 74(3) (2006) 031309.

[170] J.M. Benson, E. Snyders, The need for powder characterisation in the additive manufacturing industry and the establishment of a national facility, South African Journal of Industrial Engineering 26(2) (2015) 104-114.

[171] W.M. Visscher, M. Bolsterli, Random Packing of Equal and Unequal Spheres in Two and Three Dimensions, Nature 239 (1972) 504.

[172] A. Donev, F.H. Stillinger, S. Torquato, Do Binary Hard Disks Exhibit an Ideal Glass Transition?, Physical Review Letters 96(22) (2006) 225502.

[173] T.S. Hudson, P. Harrowell, Dense Packings of Hard Spheres of Different Sizes Based on Filling Interstices in Uniform Three-Dimensional Tilings, The Journal of Physical Chemistry B 112(27) (2008) 8139-8143.

[174] W. Schaertl, H. Sillescu, Brownian dynamics of polydisperse colloidal hard spheres: Equilibrium structures and random close packings, Journal of Statistical Physics 77(5) (1994) 1007-1025.

[175] A. Clarke, Coating on a rough surface, AIChE Journal 48(10) (2002) 2149-2156.

[176] H. Benkreira, The effect of substrate roughness on air entrainment in dip coating, Chemical Engineering Science 59(13) (2004) 2745-2751.

[177] T.J. Marshall, A RELATION BETWEEN PERMEABILITY AND SIZE

DISTRIBUTION OF PORES, Journal of Soil Science 9(1) (1958) 1-8.

[178] A. Costa, Permeability-porosity relationship: A reexamination of the KozenyCarman equation based on a fractal pore-space geometry assumption, Geophysical Research Letters 33(2) (2006) n/a-n/a.

[179] M. Lanzetta, E. Sachs, Improved surface finish in 3D printing using bimodal powder distribution, Rapid Prototyping Journal 9(3) (2003) 157-166. 
[180] F.F. Lange, Sinterability of Agglomerated Powders, Journal of the American Ceramic Society 67(2) (1984) 83-89.

[181] R. Bjørk, V. Tikare, H.L. Frandsen, N. Pryds, The Effect of Particle Size

Distributions on the Microstructural Evolution During Sintering, Journal of the American Ceramic Society 96(1) (2013) 103-110.

[182] L.C. Lim, P.M. Wong, J. Ma, Colloidal processing of sub-micron alumina powder compacts, Journal of Materials Processing Technology 67(1) (1997) 137-142.

[183] Das N, M. H., Effects of size distribution of the starting powder on the pore size and its distribution of tape cast alumina microporous membrane, J Eur Ceram Soc 19 (1999) 341-345.

[184] Y. Chaiyabutr, R. Giordano, R. Pober, The effect of different powder particle size on mechanical properties of sintered alumina, resin- and glass-infused alumina, Journal of biomedical materials research. Part B, Applied biomaterials 88(2) (2009) 502-8. [185] G. Lu, G.Q. Lu, Z.M. Xiao, Mechanical Properties of Porous Materials, Journal of Porous Materials 6(4) (1999) 359-368.

[186] S.N. Ahmad, J. Hashim, M.I. Ghazali, The Effects of Porosity on Mechanical Properties of Cast Discontinuous Reinforced Metal-Matrix Composite, Journal of Composite Materials 39(5) (2005) 451-466.

[187] X.-h. Wang, J.-s. Li, R. Hu, H.-c. Kou, L. Zhou, Mechanical properties of porous titanium with different distributions of pore size, Transactions of Nonferrous Metals Society of China 23(8) (2013) 2317-2322.

[188] T. Gambaryan-Roisman, Liquids on porous layers: wetting, imbibition and transport processes, Current Opinion in Colloid \&amp; Interface Science 19(4) (2014) 320-335.

[189] S. Jung, I.M. Hutchings, The impact and spreading of a small liquid drop on a nonporous substrate over an extended time scale, Soft Matter 8(9) (2012) 2686-2696.

[190] S. Schiaffino, A.A. Sonin, Molten droplet deposition and solidification at low Weber numbers, Physics of Fluids 9(11) (1997) 3172-3187.

[191] H. Dong, W.W. Carr, D.G. Bucknall, J.F. Morris, Temporally-resolved inkjet drop impaction on surfaces, AIChE Journal 53(10) (2007) 2606-2617.

[192] J. Fukai, Y. Shiiba, T. Yamamoto, O. Miyatake, D. Poulikakos, C.M. Megaridis, Z. Zhao, Wetting effects on the spreading of a liquid droplet colliding with a flat surface: Experiment and modeling, Physics of Fluids 7(2) (1995) 236-247.

[193] E.W. Collings, A.J. Markworth, J.K. McCoy, J.H. Saunders, Splat-quench solidification of freely falling liquid-metal drops by impact on a planar substrate, Journal of Materials Science 25(8) (1990) 3677-3682.

[194] S. Chandra, C.T. Avedisian, On the Collision of a Droplet with a Solid Surface, Proceedings of the Royal Society of London. Series A: Mathematical and Physical Sciences 432(1884) (1991) 13-41.

[195] M. Pasandideh-Fard, Y.M. Qiao, S. Chandra, J. Mostaghimi, Capillary effects during droplet impact on a solid surface, Physics of Fluids 8(3) (1996) 650-659. [196] H. Park, W.W. Carr, J. Zhu, J.F. Morris, Single drop impaction on a solid surface, AIChE Journal 49(10) (2003) 2461-2471.

[197] C. Ukiwe, A. Mansouri, D.Y. Kwok, The dynamics of impacting water droplets on alkanethiol self-assembled monolayers with co-adsorbed $\mathrm{CH} 3$ and $\mathrm{CO} 2 \mathrm{H}$ terminal groups, J Colloid Interface Sci 285(2) (2005) 760-8. 
[198] N. Reis, B. Derby, Ink Jet Deposition of Ceramic Suspensions: Modeling and Experiments of Droplet Formation, MRS Proceedings 625 (2011).

[199] M.L.M. Oostveen, G.M.H. Meesters, J.R. van Ommen, Quantification of powder wetting by drop penetration time, Powder Technology 274 (2015) 62-66.

[200] B.D. Summ, V.M. Samsonov, Concepts of Rehbinder's school and modern theories of spreading, Colloids and Surfaces A: Physicochemical and Engineering Aspects 160(2) (1999) 63-77.

[201] J. De Coninck, M.J. de Ruijter, M. Voué, Dynamics of wetting, Current Opinion in Colloid \& Interface Science 6(1) (2001) 49-53.

[202] F.T. Dodge, The spreading of liquid droplets on solid surfaces, Journal of Colloid and Interface Science 121(1) (1988) 154-160.

[203] S.F. Kistler, Hydrodynamics of wetting, in: J.C.B. (Ed.) (Ed.), Surfactant Science Series 49, Wettability, Marcel Dekker, New York 1993, pp. 311-429.

[204] P.G. de Gennes, Wetting: statics and dynamics, Reviews of Modern Physics 57(3) (1985) 827-863.

[205] A.D. 445-17a, Standard Test Method for Kinematic Viscosity of Transparent and Opaque Liquids (and Calculation of Dynamic Viscosity) ASTM, May 2017

[206] F.A.L. Dullien, M.S. El-Sayed, V.K. Batra, Rate of capillary rise in porous media with nonuniform pores, Journal of Colloid and Interface Science 60(3) (1977) 497-506. 


\section{CURRICULUM VITAE}

\section{Hadi Miyanaji}

Department of Industrial Engineering

J.B. Speed School of Engineering

University of Louisville

Louisville, KY 40292

\section{Education}

- $\quad$ Spring 2014-Summer 2018: Ph.D. in Industrial Engineering

J.B. Speed School of Engineering University of Louisville (UofL), Louisville, Kentucky

- $\quad$ Fall 2008 - Spring 2011: M.Sc. in Mechanical Engineering (Production and Manufacturing Eng.)

Iran University of Science and Technology (IUST), Tehran, Iran

- Fall 2004- Summer 2008: B.S. in Mechanical Engineering (Production and Manufacturing Eng.)

University of Tabriz (UofT), Tabriz, Iran

\section{Honors and Awards}

- UofL Doctoral Dissertation Award, Spring 2018.

- UofL-National Science Foundation I-Corps Research Award, Spring 2017

- Full Graduate Assistantship - J.B. Speed School of Engineering, Spring 2014 Present

- NSF Student Travel Awards for MSEC Conference 2015 \& 2016, and SFF Conference 2016 \& 2017

- Competition judge for National SkillsUSA Additive Manufacturing Contest (SME), June 2015

- Student Spotlight (webpage)-University of Louisville- June 2015 


\section{Publications}

\section{Journal Papers:}

- H. Miyanaji, Niknam Momenzadeh, Li. Yang, "Effect of powder material characteristics on quality of parts fabricated via binder jetting process', Rapid Prototyping Journal. [Accepted]

- H. Miyanaji, M. Orth, J. M. Akbar, L. Yang, "Process development for green part printing using binder jetting additive manufacturing" Engineering, 2018. [Full text]

- H. Miyanaji, Niknam Momenzadeh, Li. Yang, "Effect of printing speed on dimensional accuracy of printed parts via Binder Jetting Additive Manufacturing Process", Additive Manufacturing, 2017. [Full text]

- H. Miyanaji, Li. Yang, "A new physics-based model for equilibrium saturation determination in Binder Jetting Additive Manufacturing process", International Journal of Machine Tools and Manufacture, 2017. [Full text]

- L. Yang, H. Miyanaji, D. J. Ram, A. Zandinejad. S. Zhang, "Functionally Graded Ceramic Based Materials Using Additive Manufacturing: Review and Progress", Additive Manufacturing and Strategic Technologies in Advanced Ceramics: Ceramic Transactions, 2016. [Full text]

- J.J.S. Dilip, H. Miyanaji, Austin Lassell, Thomas L. Starr, Brent Stucker, “A Novel Method to Fabricate Porous TiAl intermetallic alloy 3D parts using Additive Manufacturing." Defence Technology, 2017. [Full Text]

- H. Miyanaji, S. Zhang, A. Lassell, A. Zandinejad, L. Yang, "Process Development of Porcelain Ceramic Material with Binder Jetting Process for Dental Applications", The Journal of The Minerals, Metals \& Materials Society (TMS), 2016. [Full text]

- A. K. Panahi, H. Miyanaji, E. Miandoabchi, M. H. Fareed, "Optimization of the PIM molding parameters using the sequential simplex algorithm and sensitivity analysis", Journal of Manufacturing Science and Engineering, 2013. [Full text]

- H. Bisadi, M. Rezazadeh, H. Miyanaji, M. Abdoli, "A modification on ECAP process by incorporating twist channel", Journal of Materials Engineering and Performance, 2013.[Full text] 


\section{Peer Reviewed Conference Papers}

- H. Miyanaji, L. Yang, M. J. Akbar, "Fabrication and Characterization of Graphite/Nylon 12 Composite via Binder Jetting Additive Manufacturing Process", in Proceedings of the International Solid Freeform Fabrication Symposium, Austin, TX, USA, 2017. [Full text]

- L. Yang, H. Miyanaji, "Ceramic Additive Manufacturing: A Review of Current Status and Challenges", in Proceedings of the International Solid Freeform Fabrication Symposium, Austin, TX, USA, 2017. [Full text]

- R. Hajavifard, M. Motahari, H. Özden, H. Miyanaji, and S. Kafashi, "The Effects of Pulse Shaping Variation in Laser Spot-Welding of Aluminum", in 44th North American Manufacturing Research Conference, NAMRC 44, Blacksburg, Virginia, USA, 2016. [Full text]

- H. Miyanaji, L. Yang, "Equilibrium Saturation in Binder Jetting 3D Printing Process: Theoretical Model vs. Experimental Observations", in Proceedings of the International Solid Freeform Fabrication Symposium, Austin, TX, USA, 2016. [Full text]

- H. Miyanaji, S. Zhang, A. Lassell, A. A. Zandinejad, L. Yang, "Optimal process parameters for 3D printing of dental porcelain structures", in Procedia Manufacturing, 2016. [Full text]

- S. Zhang, S. Dilip, L. Yang, H. Miyanaji, B. Stucker, "Property Evaluation of Metal Cellular Strut Structures via Powder Bed Fusion AM", in Proceedings of the International Solid Freeform Fabrication Symposium, Austin, TX, USA, 2015. [Full text]

- H. Miyanaji, L. Yang, S. Zhang, A. Zandinejad, "A preliminary study of the graded dental porcelain ceramic structures fabricated via binder jetting 3D printing", in Proceedings of the International Solid Freeform Fabrication Symposium, Austin, TX, USA, 2014. [Full text]

- S. Zhang, H. Miyanaji, L. Yang, A. A. Zandinejad, B. Stucker, "An Experimental Study of Ceramic Dental Porcelain Materials Using A 3D Print (3DP) Process", in Proceedings of the International Solid Freeform Fabrication Symposium, Austin, TX, USA, 2014. [Full text]

- H. Miyanaji, A. K. Panahi, R. Hajavifard, "Production of Ultra-Fine-Grained Aluminum Plates By Constrained Groove Pressing Technique", ASME International Mechanical Engineering Congress \& Exposition (IMECE), 2013, San Diego, CA, USA. 


\section{Presentations}

- "Fabrication and characterization of Graphite/Nylon 12 composite via BJ-AM technology", Solid Freeform Fabrication (SFF) Symposium. 2017, Austin, TX, USA.

- "Effect of printing speed on quality of printed parts in BJ-AM Process", Solid Freeform Fabrication (SFF) Symposium. 2017, Austin, TX, USA.

- Equilibrium saturation in BJ-AM process: theoretical model vs. experimental observations", Solid Freeform Fabrication (SFF) Symposium. 2016, Austin, TX, USA.

- "Optimal Process Parameters for 3D Printing of Porcelain Structures", NAMRC/MSEC Conference, 2016, Blacksburg, VA, USA.

- "Development of a Binder Jetting 3D Printing Method for Production of Dental Porcelain Ceramic Structures", NAMRC/MSEC Conference, 2015, Charlotte, $\mathrm{NC}$, USA (poster).

- "Custom Cervical Orthotic Based on Patient's Anthropometry", NAMRC/MSEC Conference, 2015, Charlotte, NC, USA (poster).

- "A preliminary study of the graded dental porcelain ceramic structures fabricated via BJ-AM process, Solid Freeform Fabrication (SFF) Symposium. 2014, Austin, TX, USA. 